\title{
A Multitracer Approach to Detecting Wastewater Plumes from Municipal Injection Wells in Nearshore Marine Waters at Kihei and Lahaina, Maui, Hawaii
}

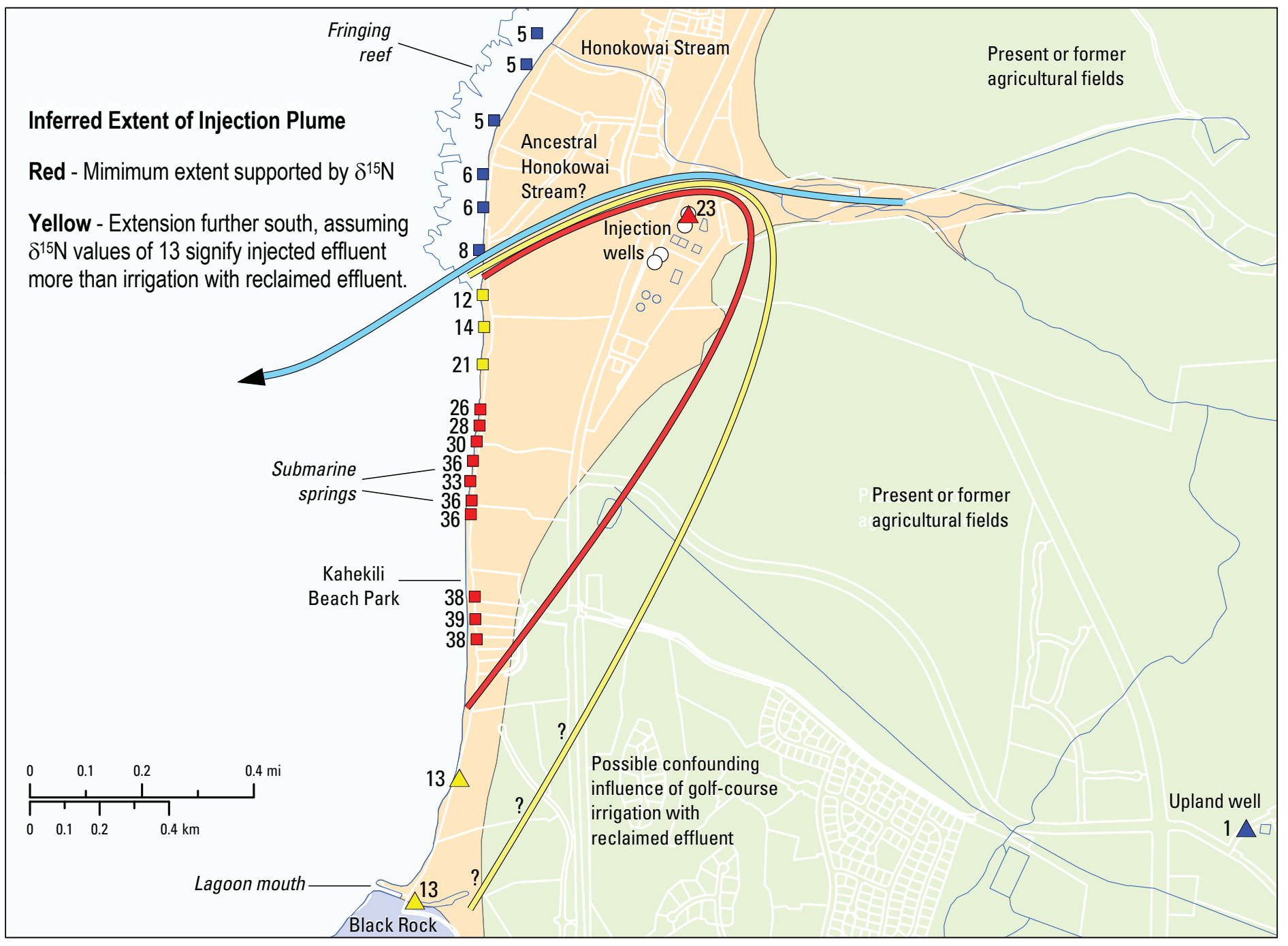

Scientific Investigations Report 2009-5253 
Cover: Lahaina wastewater injection plume inferred from $\delta^{15} \mathrm{~N}$ isotopic composition of algae (squares) and water (triangles). The plume does not take the shortest path to the coast but instead is displaced south, most likely by a buried ancestral valley of Honokowai Stream. 


\section{A Multitracer Approach to Detecting Wastewater Plumes from Municipal Injection Wells in Nearshore Marine Waters at Kihei and Lahaina, Maui, Hawaii}

By Charles D. Hunt, Jr., and Sarah N. Rosa

Prepared in Cooperation with the Hawaii State Department of Health, Clean Water Branch

Scientific Investigations Report 2009-5253 


\section{U.S. Department of the Interior \\ KEN SALAZAR, Secretary \\ U.S. Geological Survey \\ Marcia K. McNutt, Director}

\section{U.S. Geological Survey, Reston, Virginia: 2009}

This report and any updates to it are available online at:

http://pubs.usgs.gov/sir/2009/5253/

For more information on the USGS - the Federal source for science about the Earth, its natural and living resources, natural hazards, and the environment, visit http://www.usgs.gov or call 1-888-ASK-USGS

For an overview of USGS information products, including maps, imagery, and publications, visit http://www.usgs.gov/pubprod

To order this and other USGS information products, visit http://store.usgs.gov

Any use of trade, product, or firm names is for descriptive purposes only and does not imply endorsement by the U.S. Government.

Although this report is in the public domain, permission must be secured from the individual copyright owners to reproduce any copyrighted materials contained within this report.

Suggested citation:

Hunt, C.D. Jr., and Rosa, S.N., 2009, A Multitracer approach to detecting wastewater plumes from municipal injection wells in nearshore marine waters at Kihei and Lahaina, Maui, Hawaii: U.S. Geological Survey Scientific Investigations Report 2009-5253, $166 \mathrm{p}$. 


\section{Executive Summary}

Municipal wastewater plumes discharging from aquifer to ocean were detected by nearshore wading surveys at Kihei and Lahaina, on the island of Maui in Hawaii. Developed in cooperation with the Hawaii Department of Health, the survey methodology included instrument trolling to detect submarine groundwater discharge (fig. E1), followed by analysis of water and algae samples for a suite of chemical and isotopic constituents that constitute a "multitracer" approach. Following a successful proof-of-concept phase, surveys were conducted at two municipal-scale wastewater reclamation facilities on Maui, where several million gallons per day of "reclaimed water" (treated and disinfected wastewater effluent) are injected into deep wells. The main question to be answered by the study - and a topic of long-standing interest - is where and how the effluent discharges to the nearshore coastal environment.

The injected wastewater plumes were detected at both locales and with positive detection across multiple wastewater tracers in both instances. The mapped effluent plume at Kihei coincided almost exactly with prior model predictions of a mile-wide plume centered at Kalama Park (fig. E2). The plume was delineated best by the nitrogen-isotope composition of algae and water samples, with higher values being more indicative of animal or human waste nitrogen (fertilizer and soil nitrogen have values around -5 to +5 per mil). Wastewater presence was confirmed by detection of the pharmaceuticals carbamazepine (an anticonvulsant) and sulfamethoxazole (an

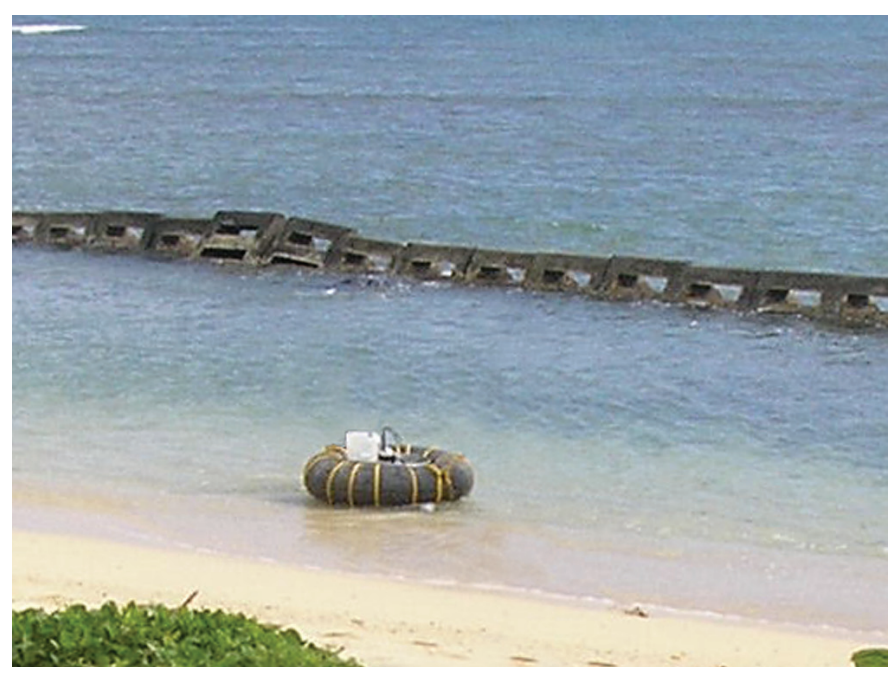

Figure E1. Photograph showing the floating inner-tube platform holding multiparameter water-quality sonde and sample bottles. (Photograph by Joshua Marvit). antibiotic) and by elevated nitrogen and phosphorus concentrations within the plume footprint.

At Lahaina, the mapped effluent plume does not extend directly shoreward from the injection wells as would generally be expected (fig. E3). Instead, the injection plume is diverted to the southwest, possibly by a buried valley fill from an ancestral stream course. Effluent injected deep into the volcanic-rock aquifer should rise buoyantly and enter overlying sediments beneath the narrow coastal plain. From there, effluent may flow preferentially through permeable limestone, gravel, and sand layers, and this may also help guide effluent to the southwest. There is some uncertainty as to how far south the Lahaina plume extends, because reclaimed wastewater is also applied as golf-course irrigation and reinfiltration of the reclaimed water may contribute to the apparent effluent signature in water chemistry near Black Rock. However the core of the effluent plume is clearly evident near Kahekili Beach Park. Wastewater presence was confirmed at submarine springs there by detection of carbamazepine and sulfamethoxazole, as well as two synthetic musk fragrances, a fire retardant, and a plasticizer compound, all of which were present in effluent sampled at the treatment plant. Nitrogen and phosphorus concentrations also were elevated within the plume footprint, as they were at Kihei.

Our survey results do not preclude effluent discharging farther offshore, where scuba reconnaissance and porewater sampling surveys might be helpful in mapping the offshore extent of effluent. For the time being, our surveys illustrate that effluent discharge is mappable as a distinct spatial pattern next to the beach.

The geochemistry of sampled waters reflects dilution of effluent by ambient groundwater and seawater, natural attenuation of nutrients in the aquifer (denitrification, sorption or mineralization of phosphorus), and biologic uptake of nutrients in the ocean. Aquifer attenuation reduces nutrient loads to coastal waters; however, further study would be necessary to quantify the nutrient loads and to better characterize the governing processes. Additional samples from onshore monitor wells and offshore porewaters could provide the foundation for such a study.

The wastewater injection plumes are not the sole source of nutrients discharging to the ocean on Maui. Groundwater beneath fertilized fields is similarly high in nitrogen as effluent, and even beneath forests groundwater nitrogen concentrations are two to three orders of magnitude higher than low-nutrient seawater. Phosphorus concentrations are considerably higher in effluent than in forest or agricultural groundwaters, however. A current assessment of nutrient loads from various land uses could be an aid in making sound nutrient-management decisions. 


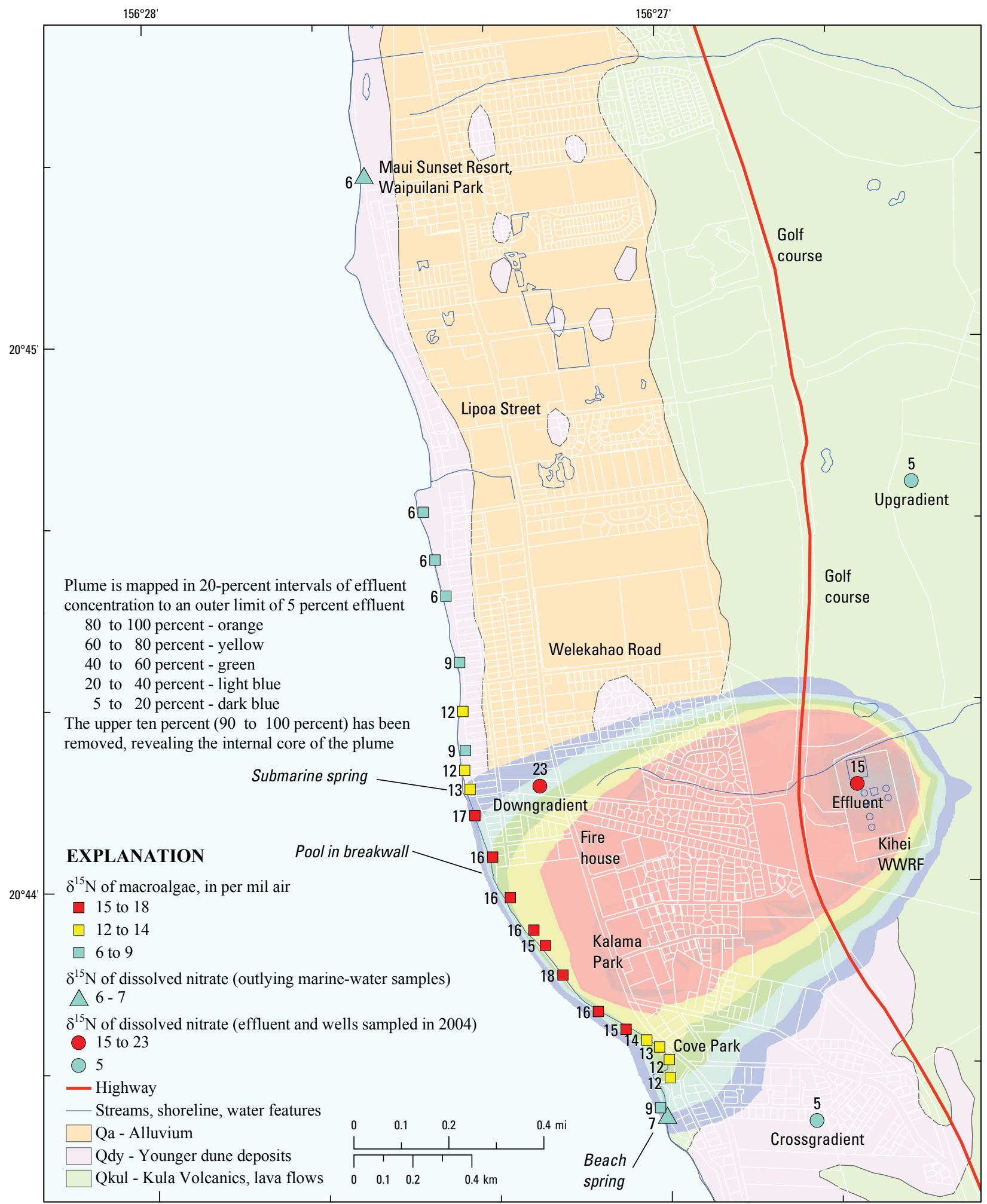

Base from U.S. Geological Survey digital data (2004) at 1:24,000 scale, UTM Zone 4, NAD83 datum. Street and property boundaries are from Hawaii State 0ffice of Planning tax maps (2008). Geology is from Stearns and Macdonald (1942) and Sherrod and others (2007).

Figure E2. Map showing correspondence between nitrogen-isotope values and the previously modeled injection plume at Kihei, Hawaii. 


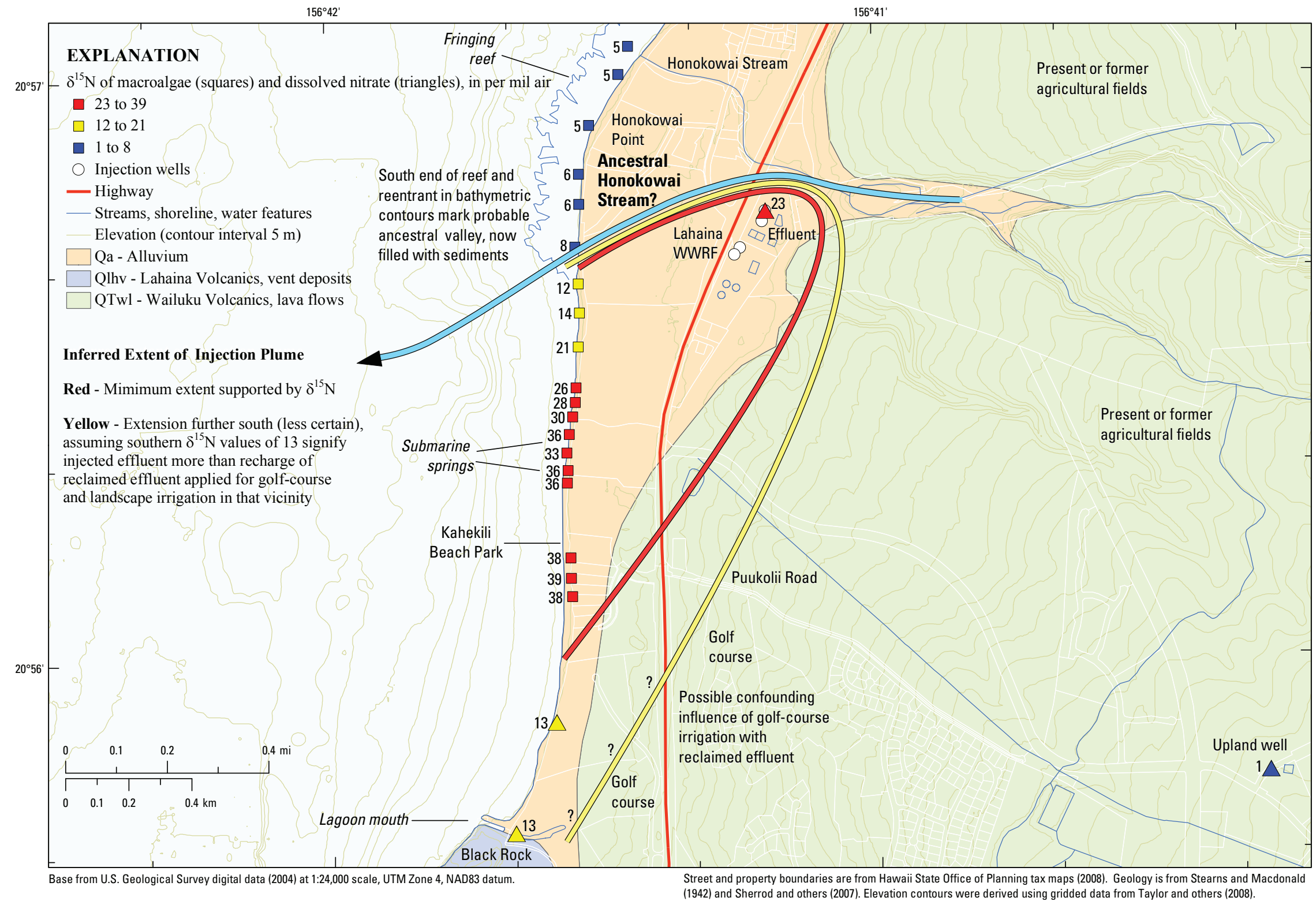

Figure E3. Map showing the injection plume diverted south by a probable buried ancestral valley at Lahaina, Hawaii. 
This page intentionally left blank 


\section{Contents}

Executive Summary .............................................................................................................................ii

Conversion Factors and Datums .............................................................................................

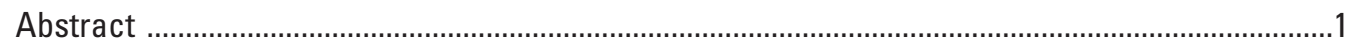

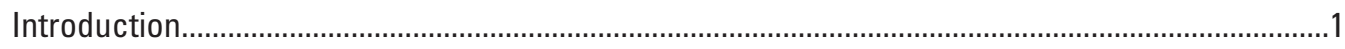

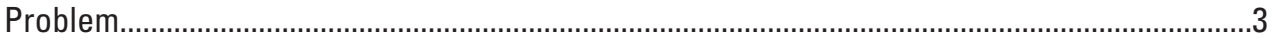

Purpose and Scope of this Report ................................................................................

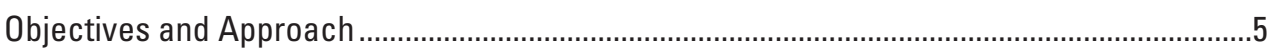

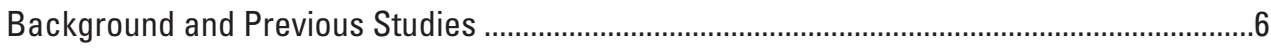

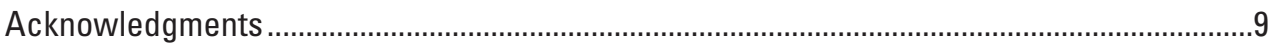

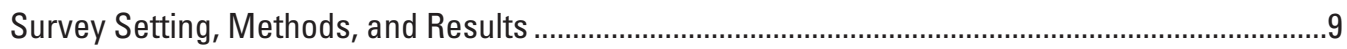

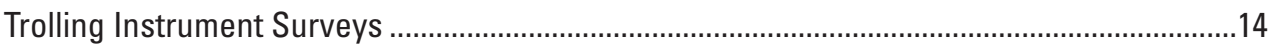

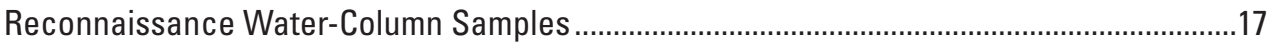

Laboratory Water-Column Samples...................................................................................

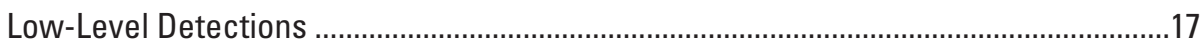

Recomputation of Reported Nutrient Concentrations .....................................................17

Seawater Unmixing Calculation to Estimate Terrestrial Source Concentration in the Fresh Fraction ......................................................................................19

Stable Isotopes and Isotope Notation..........................................................................31

Sample Collection, Processing, and Analysis..................................................................

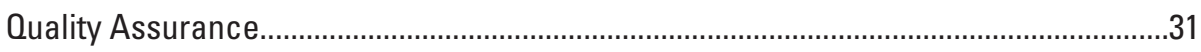

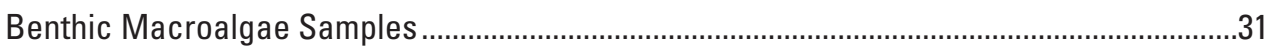

Detection and Mapping of Injected Effluent and Groundwater Discharge …….............................33

Trolling Instrument Surveys ...............................................................................................

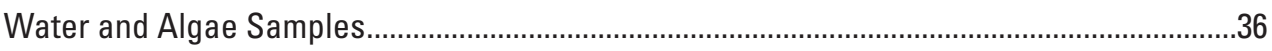

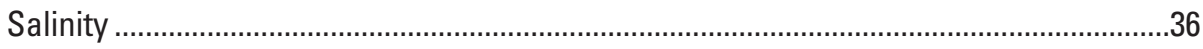

Fabric Brightener Fluorescence ................................................................................36

Pharmaceuticals and Waste Indicator Compounds .....................................................37

Nutrients, Nutrient Transformations and Processes, and Nitrogen Stable Isotopes..........41

Nutrients and Nitrogen Isotopes at Kihei ..................................................................4

Nutrients and Nitrogen Isotopes at Lahaina ...........................................................50

Hydrogen and Oxygen Stable Isotopes of Water ……….............................................55

Mixing and Chemical Evolution of Injected Effluent ...................................................................5

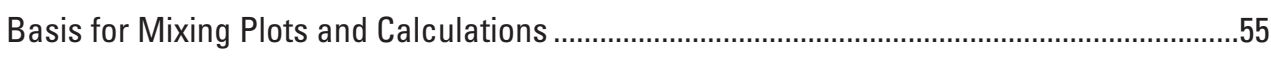

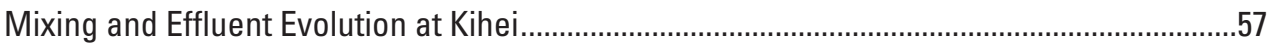

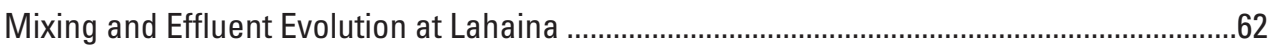

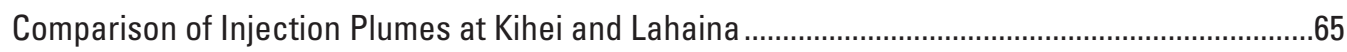

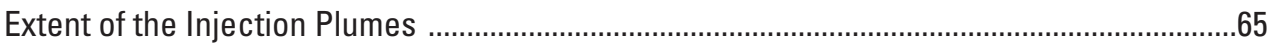

Comparison of Nutrient Attenuation at Kihei and Lahaina ...................................................68

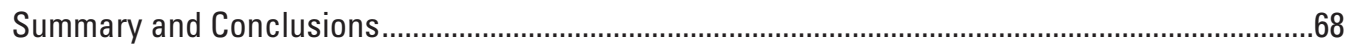

Study Limitations and Opportunities for Future Research ..........................................................69

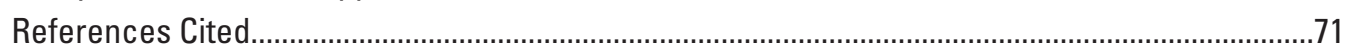

Appendix A. Maps and tables of Results from Wading and Kayak Instrument Surveys at Kihei and Lahaina, Hawaii 
Appendix B. Maps of Laboratory Results and Field Measurements for Water and Algae Samples at Kihei, Hawaii .....

Appendix C. Maps of Laboratory Results and Field Measurements for Water and Algae Samples at Lahaina, Hawaii

\section{Figures}

E1. Photograph showing the floating inner-tube platform holding multiparameter water-quality sonde and sample bottles

E2. Map showing correspondence between nitrogen-isotope values and the previously modeled injection plume at Kihei, Hawaii. iv

E3. Map showing the injection plume diverted south by a probable buried ancestral valley at Lahaina, Hawaii..

1. Map showing locations of the Kihei and Lahaina Wastewater Reclamation Facilities, Maui, Hawaii

2. Aerial photograph showing a model-simulated wastewater injection plume at Kihei, Hawaii

3. Photograph showing the floating inner-tube platform holding multiparameter water-quality sonde and sample bottles, Kualoa Beach, Oahu, Hawaii

4. Map showing locations of wastewater and nutrient source tracking field studies in Hawaii since 2006

5. Cutaway block diagram showing a modeled injection plume, Kihei, Hawaii .7

6. Aerial photograph showing fringing reef in the Kalama Park area of Kihei, Hawaii ................10

7. Aerial photograph showing fringing reef from Kahekili Beach to Honokowai Point near Lahaina, Hawaii.

8. Photograph showing the seawalls and narrow beach along a kayak survey at Kihei, Hawaii

9. Map showing locations of laboratory water samples at Kihei, Hawaii, with algal bioherms and the modeled injection plume of Hunt (2007)

10. Photograph showing the broad, steep beach along a kayak survey at Kahekili Beach Park near Lahaina, Hawaii

11. Map showing locations of laboratory water samples along Kahekili Beach near Lahaina, Hawaii...

12. Oblique aerial photograph showing the channelized drainage lagoon at Black Rock near Lahaina, Hawaii.

13. Photograph showing improvised wading platform for water-quality sonde .............................16

14. Photograph showing water-quality sonde tethered to kayak stern.........................................17

15. Map showing freshwater fraction along wading instrument survey at Kihei, Hawaii .............34

16. Map showing freshwater fraction along wading instrument survey at Lahaina, Hawaii ........35

17. Map showing pharmaceuticals in water at Kihei, Hawaii.....................................................38

18. Map showing pharmaceuticals in water at Lahaina, Hawaii ...................................................39

19. Map showing waste indicator compounds in water at Kihei, Hawaii .......................................40

20. Map showing waste indicator compounds in water at Lahaina, Hawaii...................................42

21. Graph showing nitrogen concentrations associated with different land uses and nitrogen sources, using data mostly from this study

22. Graph showing nitrogen concentration and speciation in treated wastewater effluent from the Kihei and Lahaina Wastewater Reclamation Facilities 
23. Diagram showing biogeochemical and physical-chemical processes affecting the speciation of nitrogen in aquatic systems.

24. Graph showing nitrate source classification based on nitrogen and oxygen stable-isotope compositions of dissolved nitrate. . .45

25. Map showing $\delta^{15} \mathrm{~N}$ isotopic composition of macroalgae at Kihei, Hawaii................................46

26. Map showing $\delta^{15} \mathrm{~N}$ isotopic composition of dissolved nitrate at Kihei, Hawaii .........................47

27. Map showing dissolved inorganic nitrogen (DIN) in water at Kihei, Hawaii ............................48

28. Map showing $\delta^{15} \mathrm{~N}$ isotopic composition of macroalgae at Lahaina, Hawaii ............................51

29. Map showing $\delta^{15} \mathrm{~N}$ isotopic composition of dissolved nitrate at Lahaina, Hawaii ...................52

30. Map showing dissolved inorganic nitrogen (DIN) in water at Lahaina, Hawaii .......................53

31. Seawater mixing plot showing $\delta^{2} \mathrm{H}$ versus salinity at Kihei, Hawaii........................................57

32. Seawater mixing plots for $(A)$ dissolved inorganic nitrogen, and $(B)$ orthophosphate in water samples at Kihei, Hawaii ..........................................................5

33. Seawater mixing plots for $(A)$ dissolved inorganic nitrogen (DIN), and (B) source DIN ..........59

34. Isotope mixing and evolution plots for $(A) \delta^{18} 0$ versus $\delta^{15} \mathrm{~N}$ of dissolved nitrate, and $(B)$ source DIN versus $\delta^{15} \mathrm{~N}$ of dissolved nitrate in water samples at Kihei, Hawaii ...............60

35. Seawater mixing plot showing $\delta^{2} \mathrm{H}$ versus salinity at Lahaina, Hawaii...................................61

36. Seawater mixing plots for $(A)$ dissolved inorganic nitrogen, and $(B)$ orthophosphate in water samples at Lahaina, Hawaii

37. Seawater mixing plots for $(A)$ dissolved inorganic nitrogen (DIN), and $(B)$ source DIN in water samples at Lahaina, Hawaii

38. Isotope evolution and mixing plots for $(A) \delta^{18} 0$ versus $\delta^{15} \mathrm{~N}$ of dissolved nitrate, and $(B)$ source DIN versus $\delta^{15} \mathrm{~N}$ of dissolved nitrate in water samples at Lahaina, Hawaii

39. Map showing correspondence between $\delta^{15} \mathrm{~N}$ and the modeled injection plume of Hunt (2007) at Kihei, Hawaii

40. Map showing injection plume inferred from $\delta^{15} \mathrm{~N}$, and southward diversion of the plume by a probable buried ancestral valley at Lahaina, Hawaii.

41. Graph comparing denitrification of effluent and upland groundwater at Kihei and Lahaina, Hawaii

A1. Freshwater fraction along wading survey at Kihei, Hawaii.....................................................78

A2. Freshwater fraction along kayak survey at Kihei, Hawaii ........................................................79

A3. Water temperature along wading survey at Kihei, Hawaii.........................................................8

A4. Water temperature along kayak survey at Kihei, Hawaii........................................................ 81

A5. Dissolved oxygen saturation along wading survey at Kihei, Hawaii .........................................82

A6. $\mathrm{pH}$ along wading survey at Kihei, Hawaii ...........................................................................83

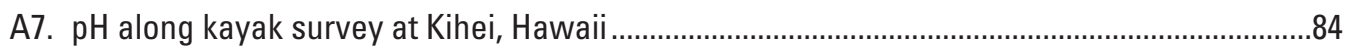

A8. Chlorophyll-a concentration along wading survey at Kihei, Hawaii ........................................85

A9. Chlorophyll-a concentration along kayak survey at Kihei, Hawaii..............................................86

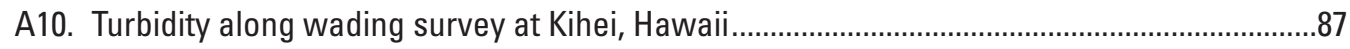

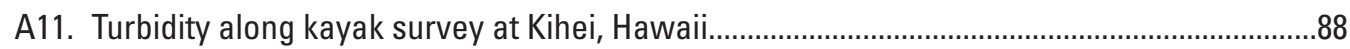

A12. Freshwater fraction (computed from salinity) along wading survey at Lahaina, Hawaii......89

A13. Water temperature along wading survey at Lahaina, Hawaii................................................90

A14. Water temperature along kayak survey at Lahaina, Hawaii .................................................91

A15. Dissolved oxygen saturation along kayak survey at Lahaina, Hawaii....................................92

A16. pH along wading survey at Lahaina, Hawaii .......................................................................93

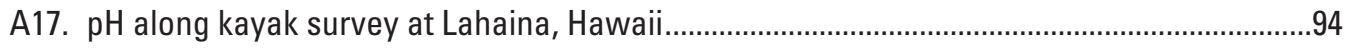

A18. Chlorophyll-a concentration along wading survey at Lahaina, Hawaii.................................95 
A19. Turbidity along wading survey at Lahaina, Hawaii.................................................................96

B1. Reconnaissance water samples at Kihei, Hawaii............................................................128

B2. Laboratory water samples (with remarks on their field context) at Kihei, Hawaii ................129

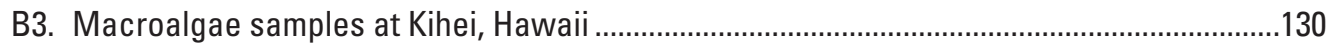

B4. Freshwater fraction (computed from salinity) in reconnaissance water samples at Kihei, Hawaii

B5. Freshwater fraction (computed from salinity) in laboratory water samples at Kihei, Hawaii ..... .132

B6. Fabric brightener fluorescence in reconnaissance water samples at Kihei, Hawaii..........133

B7. Fabric brightener fluorescence in laboratory water samples at Kihei, Hawaii ...................134

B8. Pharmaceutical compounds in water at Kihei, Hawaii .....................................................135

B9. Waste indicator compounds in water at Kihei, Hawaii.........................................................136

B10. $\delta^{15} \mathrm{~N}$ isotopic composition of macroalgae at Kihei, Hawaii ............................................137

B11. Nitrogen mass fraction in macroalgae at Kihei, Hawaii ...................................................138

B12. $\delta^{15} \mathrm{~N}$ isotopic composition of dissolved nitrate at Kihei, Hawaii .........................................139

B13. Dissolved inorganic nitrogen (DIN) in water at Kihei, Hawaii............................................. 140

B14. Nitrate plus nitrite in water at Kihei, Hawaii ...............................................................141

B15. Ammonium in water at Kihei, Hawaii ........................................................................142

B15. Ammonium in water at Kihei, Hawaii ............................................................................ 142

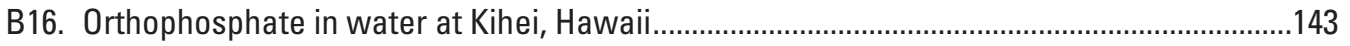

B17. Nitrogen-to-phosphorus molar ratio in water at Kihei, Hawaii............................................144

B18. $\delta^{2} \mathrm{H}$ isotopic composition of water at Kihei, Hawaii ............................................................. 145

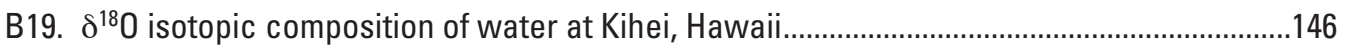

C1. Reconnaissance water samples at Lahaina, Hawaii...........................................................148

C2. Laboratory water samples (with remarks on their field context) at Lahaina, Hawaii ..........149

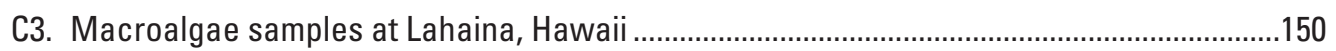

C4. Freshwater fraction (computed from salinity) in reconnaissance water samples

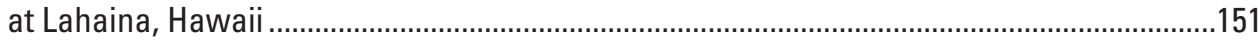

C5. Freshwater fraction (computed from salinity) in laboratory water samples

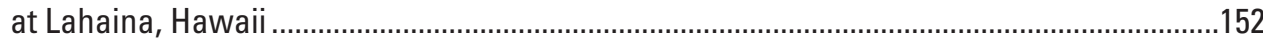

C6. Fabric brightener fluorescence in reconnaissance water samples at Lahaina, Hawaii.....153

C7. Fabric brightener fluorescence in laboratory water samples at Lahaina, Hawaii ...............154

C8. Pharmaceutical compounds in water at Lahaina, Hawaii ...................................................155

C9. Waste indicator compounds in water at Lahaina, Hawaii....................................................156

C10. $\delta^{15} \mathrm{~N}$ isotopic composition of macroalgae at Lahaina, Hawaii .............................................157

C11. Nitrogen mass fraction in macroalgae at Lahaina, Hawaii ................................................158

C12. $\delta^{15} \mathrm{~N}$ isotopic composition of dissolved nitrate at Lahaina, Hawaii ..................................159

C13. Dissolved inorganic nitrogen (DIN) in water at Lahaina, Hawaii........................................160

C14. Nitrate plus nitrite in water at Lahaina, Hawaii ................................................................161

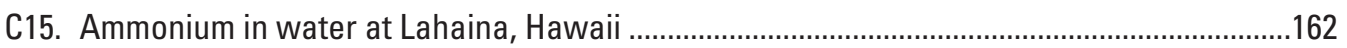

C16. Orthophosphate in water at Lahaina, Hawaii...................................................................163

C17. Nitrogen-to-phosphorus molar ratio in water at Lahaina, Hawaii..........................................164

C18. $\delta^{2} \mathrm{H}$ isotopic composition of water at Lahaina, Hawaii .........................................................165

C19. $\delta^{18} 0$ isotopic composition of water at Lahaina, Hawaii......................................................166 


\section{Tables}

1. Laboratory methods and field measurements for water and algae at Kihei and Lahaina, Hawaii, May 6-28, 2008...

2. Field measurements on reconnaissance water samples collected at Kihei and Lahaina, Hawaii, May 7-8, 2008

3. Nutrients, stable isotopes, and physical parameters for water samples collected at Kihei, Hawaii, May 20, 2008, and November 15-17, 2004.

4. Nutrients, stable isotopes, and physical parameters for water samples collected at Lahaina, Hawaii, May 21, 2008

5. Pharmaceuticals and waste indicator compounds in water samples collected at Kihei, Hawaii, May 20, 2008, and November 15-17, 2004

6. Pharmaceuticals and waste indicator compounds in water samples collected at Lahaina, Hawaii, May 21, 2008

7. Nitrogen stable isotope composition and nitrogen dry-weight concentration in Ulva sp. macroalgae samples collected at Kihei and Lahaina, Hawaii, May 7- 28, 2008........32

A1. Nearshore wading survey with multiparameter water-quality sonde at Kihei, Hawaii, May 8, 2008

A2. Nearshore kayak survey with multiparameter water-quality sonde at Kihei, Hawaii, May 22, 2008 108

A3. Nearshore wading survey with multiparameter water-quality sonde at Lahaina, Hawaii, May 6, 2008. .115

A4. Nearshore kayak survey with multiparameter water-quality sonde at Lahaina, Hawaii, May 23, 2008. 


\section{Conversion Factors and Datums}

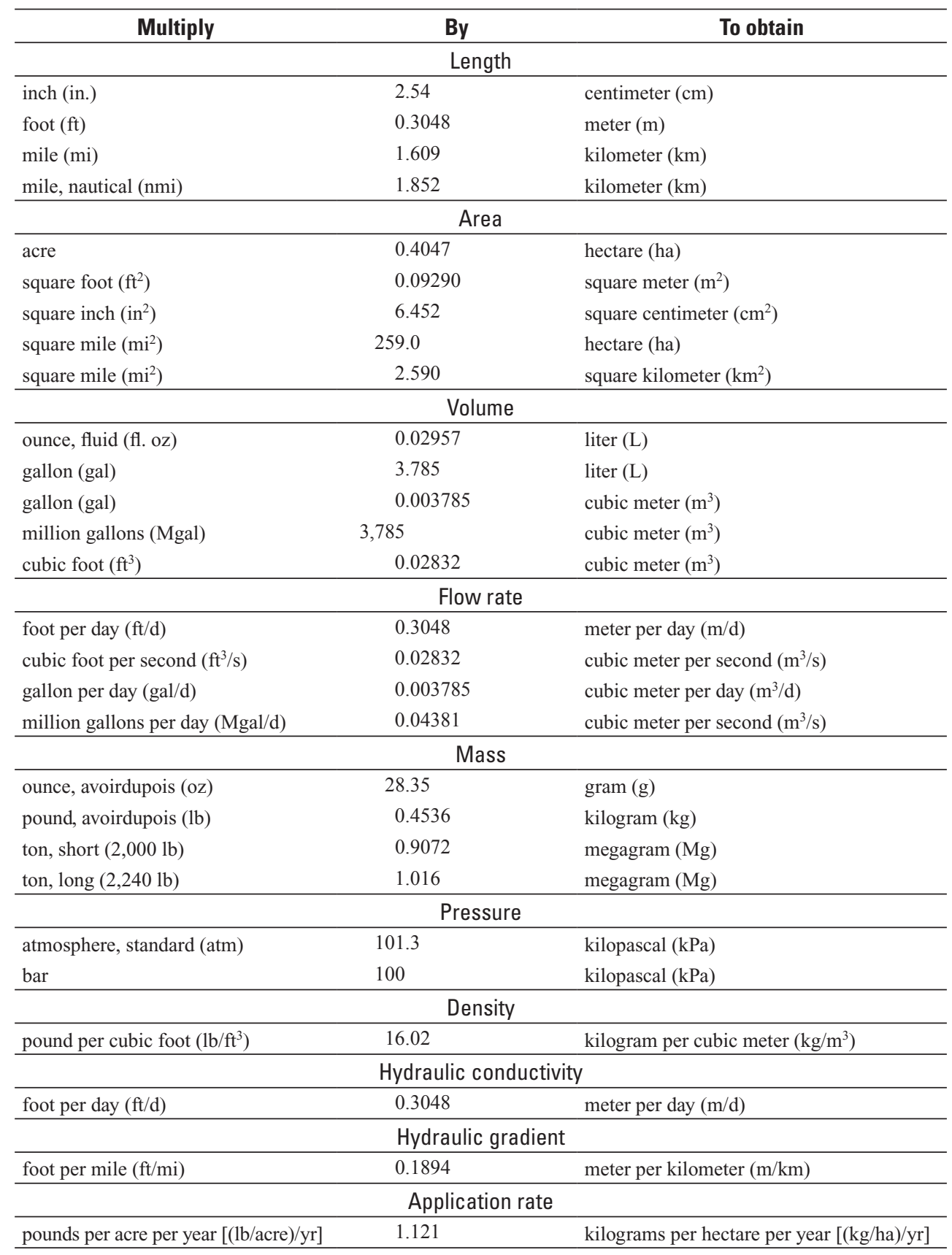

Temperature in degrees Celsius $\left({ }^{\circ} \mathrm{C}\right)$ may be converted to degrees Fahrenheit $\left({ }^{\circ} \mathrm{F}\right)$ as follows: ${ }^{\circ} \mathrm{F}=\left(1.8 x^{\circ} \mathrm{C}\right)+32$

Temperature in degrees Fahrenheit $\left({ }^{\circ} \mathrm{F}\right)$ may be converted to degrees Celsius $\left({ }^{\circ} \mathrm{C}\right)$ as follows: ${ }^{\circ} \mathrm{C}=\left({ }^{\circ} \mathrm{F}-32\right) / 1.8$

Vertical coordinate information is referenced to local mean sea level tidal datum.

Horizontal coordinate information is referenced to the North American Datum of 1983 (NAD 83).

Specific conductance is given in microsiemens per centimeter at 25 degrees Celsius $\left(\mu \mathrm{S} / \mathrm{cm}\right.$ at $\left.25^{\circ} \mathrm{C}\right)$.

Concentrations of chemical constituents in water are reported either in milligrams per liter $(\mathrm{mg} / \mathrm{L})$, micrograms per liter $(\mu \mathrm{g} / \mathrm{L})$, or micromoles per liter $(\mu \mathrm{M} / \mathrm{L})$.

Isotopic compositions are reported in delta notation, in units of per mil (parts per thousand, symbol \%o). 


\title{
A Multitracer Approach to Detecting Wastewater Plumes from Municipal Injection Wells in Nearshore Marine Waters at Kihei and Lahaina, Maui, Hawaii
}

\author{
By Charles D. Hunt, Jr., and Sarah N. Rosa
}

\section{Abstract}

Municipal wastewater plumes discharging from aquifer to ocean were detected by nearshore wading surveys at Kihei and Lahaina, on the island of Maui in Hawaii. Developed in cooperation with the Hawaii State Department of Health, the survey methodology included instrument trolling to detect submarine groundwater discharge, followed by analysis of water and macroalgae for a suite of chemical and isotopic constituents that constitute a "multitracer" approach. Surveys were conducted May 6-28, 2008, during fair-weather conditions and included: (1) wading and kayak trolling with a multiparameter water-quality sonde, (2) marine water-column sampling, and (3) collection of benthic algae samples. Instrument trolling helped guide the water sampling strategy by providing dense, continuous transects of water properties on which groundwater discharge zones could be identified. Water and algae samples for costly chemical and isotopic laboratory analyses were last to be collected but were highly diagnostic of wastewater presence and nutrient origin because of low detection levels and confirmation across multiple tracers. Laboratory results confirmed the presence of wastewater constituents in marine water-column samples at both locales and showed evidence of modifying processes such as denitrification and mixing of effluent with surrounding groundwater and seawater. Carbamazepine was the most diagnostic pharmaceutical, detected in several marine water-column samples and effluent at both Kihei and Lahaina. Heavy nitrogen-isotope compositions in water and algae were highly diagnostic of effluent, particularly where enriched to even heavier values than effluent source compositions by denitrification. Algae provided an added advantage of time-integrating their nitrogen source during growth. The measured Kihei plume coincided almost exactly with prior model predictions, but the Lahaina plume was detected well south of the expected direct path from injection wells to shore and may be guided by a buried valley fill from an ancestral course of Honokowai Stream. Nutrient concentrations in upland wells at Lahaina were comparable to concentrations in wastewater but originate instead from agricultural fertilizers. A key factor in detecting and mapping the wastewater plumes was sampling very close to shore (mostly within $20 \mathrm{~m}$ or so) and in very shallow water (mostly 0.5 to $2 \mathrm{~m}$ depth). Effluent probably discharges somewhat offshore as well, although prior attempts to detect an injected fluorescent tracer at Lahaina in the 1990s were inconclusive, having focused farther offshore in water mostly 10-30 m deep. Sampling of benthic porewater and algae would offer the best chances for further effluent detection and mapping offshore, and sampling of onland monitor wells could provide additional understanding of geochemical processes that take place in the effluent plumes and bring about some degree of natural attenuation of nutrients.

\section{Introduction}

The Kihei and Lahaina areas are popular resort destinations in south and west Maui, Hawaii (fig. 1). Hotels and condominiums line much of the shore in these areas, intermixed with suburban bedroom communities of year-round residents. The economy is largely tourism based in both areas, with visitors coming to enjoy the warm climate, sandy beaches, and ocean recreation. Upland slopes are occupied at Kihei by dryland forest with low-intensity ranching and farming and at Lahaina by former high-intensity sugarcane and pineapple fields that are now fallow, converted to lower intensity diversified-crop farming, or converted to urban use (typically resort or residential). Forest preserves lie farther upslope at high elevations in both areas, and both areas contain several golf courses.

Groundwater near the coast in both areas is brackish and not suitable for household use. Drinking water is imported to Kihei from the north side of the island by pipeline and is supplied at Lahaina by public supply wells upslope. Largecapacity irrigation wells have historically supplied water for intensive agriculture in the Lahaina area, although pumpage has tapered off dramatically since the end of sugarcane cultivation in 1999. 


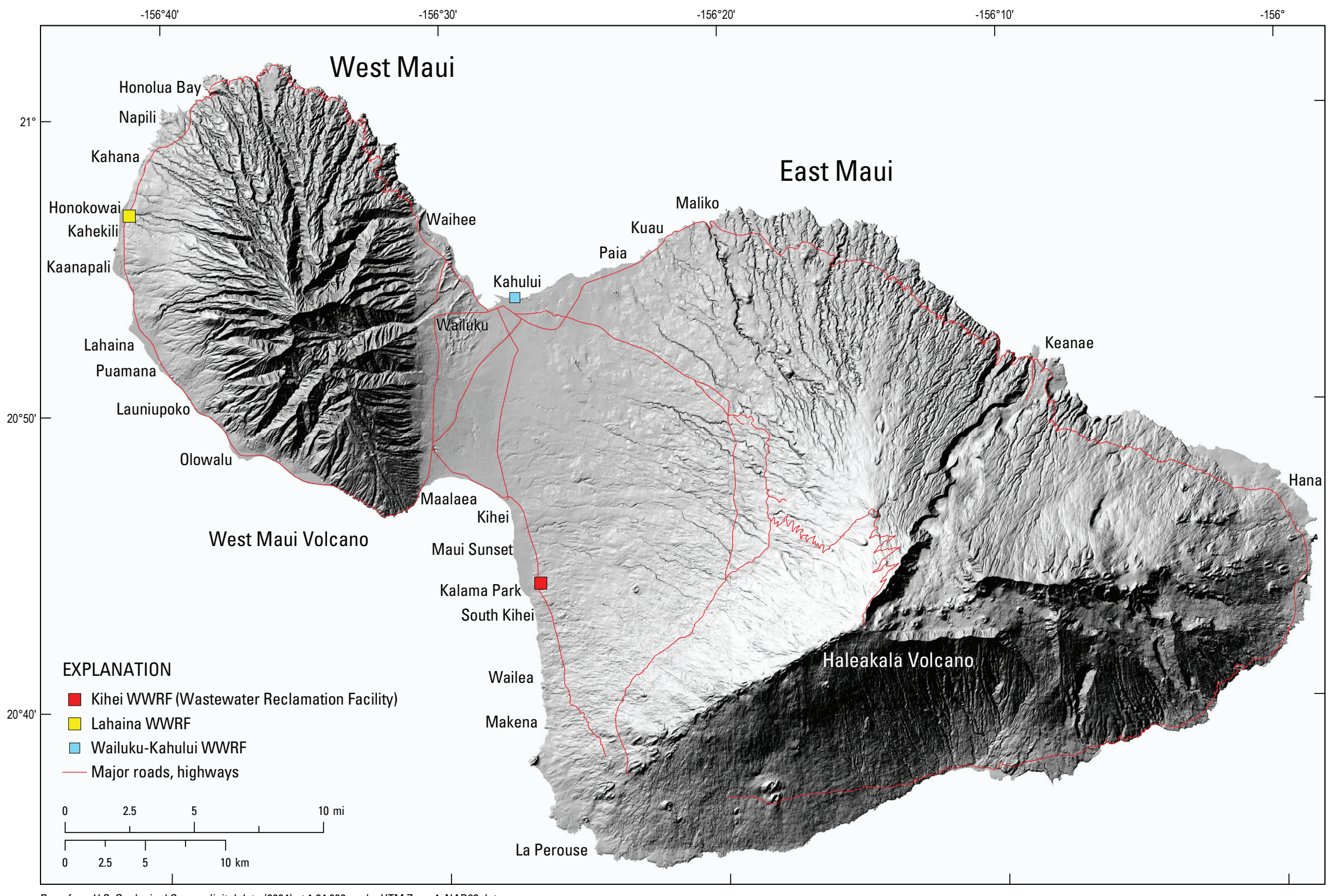

Figure 1. Map showing locations of the Kihei and Lahaina Wastewater Reclamation Facilities, Maui, Hawaii (a third County plant serves Wailuku-Kahului). 
The study areas are sewered, although there are a few isolated subdivisions remaining on cesspools and some resorts handle their own sewage treatment and disposal (though far fewer than in the 1970s and 1980s, before sewers were completed). Community wastewater is collected at the centralized Kihei and Lahaina Wastewater Reclamation Facilities (WWRFs), which are municipal-scale treatment plants operated by the County of Maui. Wastewater is treated to R1 disinfected tertiary and R2 disinfected secondary levels, producing reclaimed water that can be reapplied safely as landscape irrigation, with certain restrictions (Limtiaco Consulting Group, 2005; Parabicoli, 2007). Reclaimed water is piped to golf courses, parks, schools, and other customers for reuse as lawn and landscape irrigation. Unused effluent is disposed of by injection wells. All effluent (both reused and injected) receives final disinfection. Kihei effluent is entirely R1 quality and is disinfected by high-intensity UV (ultraviolet) light. At Lahaina, R1 water for reuse receives dual disinfection by UV and chlorination, whereas injected R2 water is chlorinated only. All effluent at a third facility at Kahului is injected and is R2 water disinfected by chlorination only (Steve Parabicoli, County of Maui Water Recycling Program Coordinator, oral commun., 2009). Although reuse programs are well established and the County strives to maximize reuse, current distribution capacities limit reuse of reclaimed water to about one-third at Kihei and one-fifth at Lahaina.

Injection of treated, disinfected effluent has averaged about $3 \mathrm{Mgal} / \mathrm{d}$ (million gallons per day) at Kihei and 4 $\mathrm{Mgal} / \mathrm{d}$ at Lahaina in recent years, and total inflows to the plants have averaged around $4.5 \mathrm{Mgal} / \mathrm{d}$ at Kihei and $5 \mathrm{Mgal} / \mathrm{d}$ at Lahaina (records of the County of Maui Division of Environmental Management). Once injected into the aquifer, effluent spreads out and flows to the coast, typically in a horseshoeshaped plume embedded in the regional groundwater flow from uplands to the sea. Volumetrically, injection is on a par with regional groundwater flow, which is mostly displaced around the plumes, although there is some mixing along plume margins and with underlying saltwater in the aquifer. Figure 2 shows a simulated plume at Kihei, which discharges over roughly a mile of coast (Hunt, 2007).

\section{Problem}

Water-quality concerns associated with the wastewater injection range from ecologic (possible effects of high-nutrient effluent on the marine ecosystem) to public health (to swimmers, if disinfection is not entirely effective). Threats to drinking water are not a concern because underlying groundwater is brackish and injection is seaward of the Underground Injection Control (UIC) line established by the State of Hawaii and the U.S. Environmental Protection Agency to protect drinking-water resources. Injection directs high-nutrient treated wastewater to the coast, but it does so within a larger shoreward regional flux of groundwater carrying background nutrients and fertilizer-enriched nutrients that in some areas are comparable to concentrations in wastewater. Resource managers and stakeholders require methods for identifying and discriminating sources of nutrients and other pollution so that proper corrective and preventive measures can be applied.

The Hawaii State Department of Health $(\mathrm{HDOH})$ Clean Water Branch administers various monitoring programs in nearshore coastal waters and stream watersheds (Hawaii State Department of Health, 2006a,b). These include the Federal Clean Water Act, enacted to ensure that waters are "fishable and swimmable," and the Beaches Environmental Assessment and Coastal Health (BEACH) Act, enacted to ensure public health during recreational exposure (U.S. Environmental Protection Agency, 2006).

HDOH monitors nutrients, suspended sediment, fecalindicator bacteria, and supporting water-quality parameters in streams and nearshore waters. Fecal-indicator bacteria are monitored as an indication of possible risk from bacterial and viral pathogens; high bacteria counts are sometimes observed, but origins of the bacteria are ambiguous without other supporting tests or mapping. Sewage or septic effluents often are the first sources to be suspected, but wildlife also can be a source of the bacteria, as can nonpoint runoff of pet and wildlife fecal matter from the watershed. Complicating matters, fecal-indicator bacteria once thought to signify proximal fecal pollution have been found to grow actively in tropical soils (Fujioka and Byappanahalli, 2003). Moreover, regular monitoring is done only at a few scattered sites, which limits the ability to infer a cause or source of the bacteria from a spatial pattern in measured data.

A coastal ecology issue of long-standing concern is the delivery of terrestrial (land-derived) nutrients to coastal waters. Sections of the Kihei and Lahaina coasts have been designated as impaired water bodies or "Water Quality Limited Segments" for exceeding one or more water-quality criteria, such as nitrogen, turbidity, or suspended sediment (Hawaii State Department of Health, 2004, 2006c). Sources contributing nitrogen and phosphorus include stormwater runoff, sewage effluents (injected, septic, cesspool, leaking sewer lines, and spills at transfer stations), agricultural and urban fertilizers, and nonpoint background sources such as natural soil fixation, decomposition of forest litter, and atmospheric deposition.

Terrestrial nutrients are one of a number of factors likely contributing to algal growth and degradation of coral reefs. Excessive growth of macroalgae (seaweed) has been a recurring problem in Maui waters since at least the late 1980s (West Maui Watershed Management Advisory Committee, 1997). Potential contributing factors include introduction of alien algae species, population reductions in algal grazers such as fish and urchins, and addition of nutrients from terrestrial sources (named above) and marine sources (oceanic currents, upwelling, gravity waves, sediment resuspension). Some investigators have inferred a pronounced proximity relation between abundant algal growth and wastewater injection at several Maui localities (Hawaii Department of Land and Natural Resources, Division of Aquatic Resources, and Hawaii Coral Reef Initiative Research Program, 


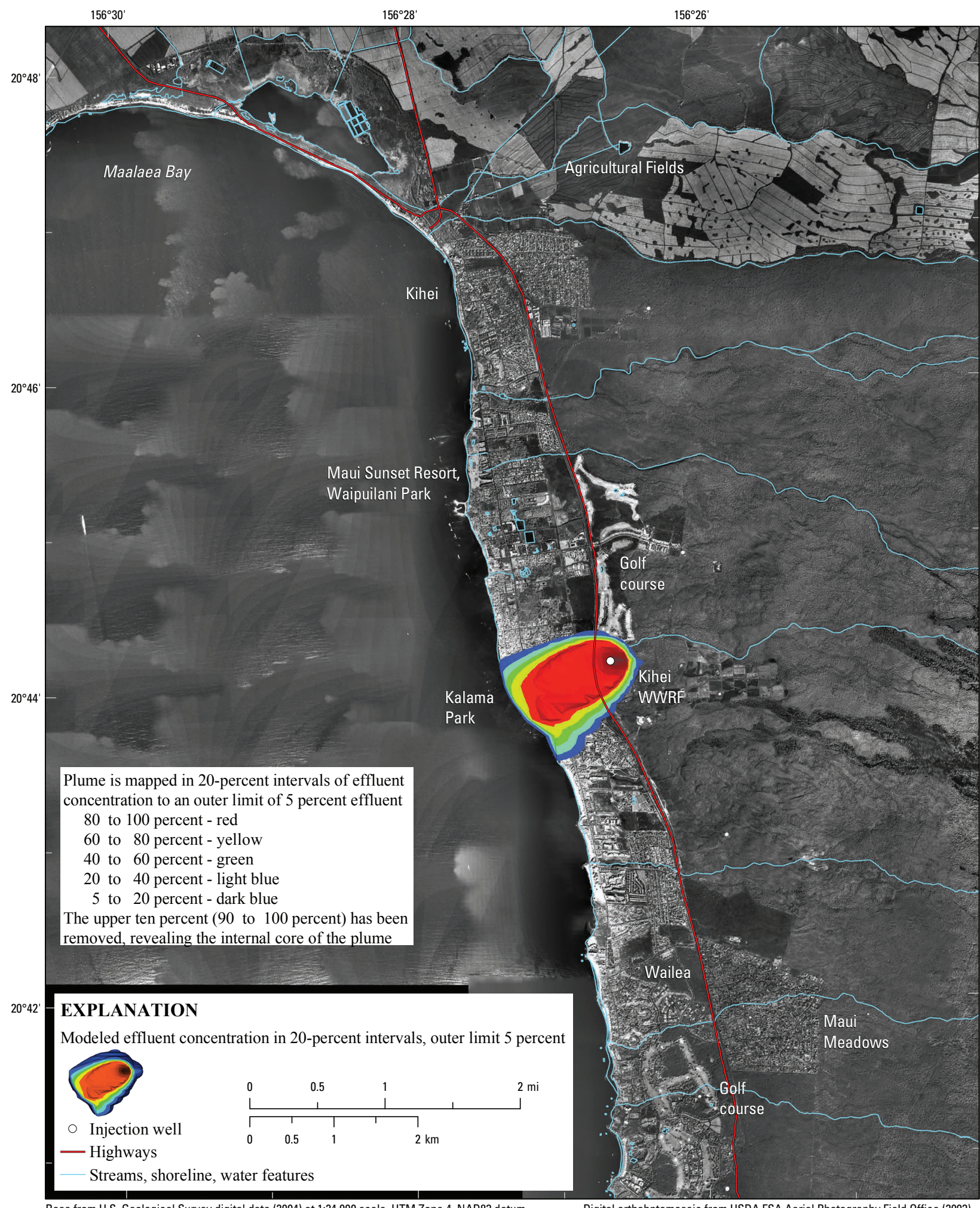

Base from U.S. Geological Survey digital data (2004) at 1:24,000 scale, UTM Zone 4, NAD83 datum.

Digital orthohptomosaic from USDA FSA Aerial Photography Field Office (2003).

Figure 2. Aerial photograph showing a model-simulated wastewater injection plume at Kihei, Hawaii (modified from Hunt, 2007). 
2007). However moderate to large accumulations of algae have been documented at beaches spanning tens of miles of coastline on Maui, a much greater extent than that of the relatively limited municipal injection plumes (Bourke, 1996; Hodges, 1996; West Maui Watershed Management Advisory Committee, 1997; Hurley, 2001; Carroll, 2004). To the extent that terrestrial nutrients contribute to algal growth in outlying areas, sources other than waste injection would be involved, such as fertilizers, cesspools, and possibly even background nutrient loads originating from upland forests.

\section{Purpose and Scope of this Report}

The purpose of this report is to convey results of nearshore wading surveys for nutrients and wastewater tracers along beaches near Kihei and Lahaina, Maui. The study was designed and funded jointly by the U.S. Geological Survey (USGS) and Hawaii State Department of Health, which locally administers BEACH Act monitoring on behalf of the U.S. Environmental Protection Agency (USEPA).

This report includes nutrient and isotopic evidence for detection of treated wastewater emerging from aquifer to marine waters shoreward of the Kihei and Lahaina wastewater reclamation facilities. It reports nutrient concentrations, constrains the lateral limits of the effluent plumes, and provides evidence of active nutrient attenuation processes such as denitrification and sorption, mixing of effluent with groundwater and seawater, and biologic uptake of nutrients. This report does not determine causes of macroalgal blooms nor the role of nutrients in macroalgal growth.

\section{Objectives and Approach}

This reconnaissance mapping study attempted first-order detection and discrimination of treated wastewater injected into the aquifer. Objectives were to (1) refine instrumental and sampling methods under development since 2006 for wastewater and nutrient source tracking; (2) conduct field surveys across major municipal wastewater injection plumes at Kihei and Lahaina to attempt detection; and (3) synthesize and report the results. Beyond these basic objectives, chemical data collected during the surveys also allow a good deal analysis of contaminant sources; mixing among effluent, ambient groundwater, and seawater; and apparent nutrient transformations and attenuation taking place naturally in the aquifer.

HDOH and USGS have cooperated to develop a "Wastewater and Nutrient Source Tracking Methodology" for beach and watershed monitoring. It provides a nearshore mapping capability using water-quality instruments and sampling for chemical tracers of wastewater and nutrient origin. The approach supplements HDOH monitoring by enhancing capabilities for pollution source discrimination while requiring only a modest level of technical expertise and readily available chemical analyses from USGS laboratories. It can be used as a screening methodology to identify and prioritize sites for more

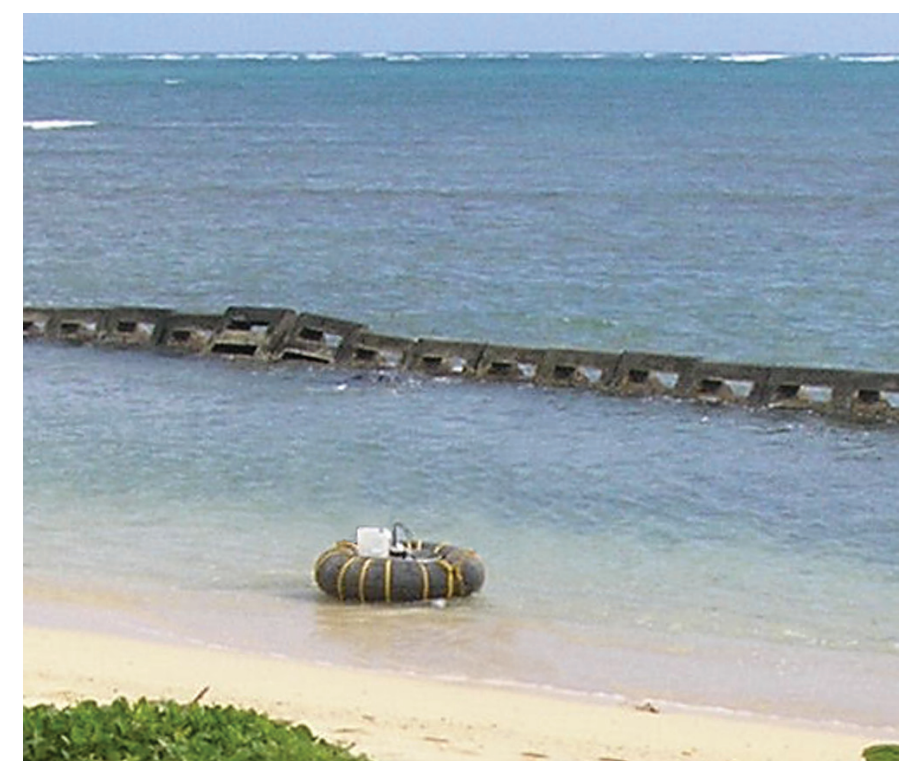

Figure 3. Photograph showing the floating inner-tube platform holding multiparameter water-quality sonde and sample bottles, Kualoa Beach, Oahu, Hawaii. (Photograph by Joshua Marvit.)

specialized approaches, for example biologic or genetic assays capable of distinguishing human sources of bacteria from animal sources.

The methodology emphasizes a mapping approach in the hopes that spatial patterns will be evident and offer a clear answer to questions posed. Global positioning system (GPS) receivers are operated in recording mode while trolling a water-quality sonde along continuous transects just off the beach (fig. 3). Position fixes also are obtained for bottled water samples analyzed for a wide variety of constituents diagnostic of wastewater presence and nutrient origin. These may be thought of as "inherent tracers" - constituents already present in water or wastewater effluent that can simply be sought by laboratory testing. This is in contrast to an "introduced tracer" - such as fluorescent dye - that is injected at a source and then sought by sampling in destination waters at some later time (how much later one should look can be difficult to estimate).

The methodology is a "multitracer" approach in that concordance across several constituents provides a measure of internal agreement and enhances confidence that detection or source inferences are conclusive. Highly diagnostic tracers for wastewater include (1) pharmaceuticals that are used and excreted by humans and present in wastewater; (2) "waste indicator compounds" such as soaps, fragrances, and household cleaners washed down the drain during bathing and cleaning; and (3) nitrogen stable isotopes, whose tracing utility is based on $\delta^{15} \mathrm{~N}$ being heavier in animal/human waste nitrogen than in fertilizers or nitrogen fixed in soil. Pharmaceuticals and waste indicators have received much attention in recent years as a new group of "emerging contaminants" (see 
for example, Kolpin and others, 2002; Glassmeyer and others, 2005). Their presence in streams and rivers raises concerns about drinking-water intakes and endocrine-disrupting effects on stream biota. However, in the present study, they are simply used as tracers or indicators of wastewater presence.

Proof-of-concept surveys in 2006 and 2007 at Kualoa Beach and Kahana Bay on Oahu (fig. 4) identified localized groundwater discharge but did not yield tracer "hotspots" near restroom septic systems suspected of contributing to high beach bacteria counts (Hunt, 2008). Based on these findings, attention was shifted from the restrooms to nonpoint pollution from the surrounding watershed (particularly during storm runoff, which is when the highest bacteria counts have been measured) and to a lesser extent to beach-house septic systems well away from the park restrooms. Subsequent surveys included a municipal wastewater disposal setting at Kealakehe, near Kailua-Kona on Hawaii Island, and the urban Kaelepulu estuary at Kailua, Oahu. The Maui municipal injection plumes have been of high interest for decades and were the next candidates selected for surveying with the newly developed methods.

\section{Background and Previous Studies}

Studies most relevant to this one include several dealing specifically with Maui wastewater injection and others that describe geology, hydrology, and water quality in the study areas. Some reports list nutrient concentrations or estimated nutrient loads, and many of these were motivated by interest in the role of nutrients in algal blooms along Maui shores. A few studies attempted detection of wastewater offshore, much like the present study.

County of Maui records, engineering reports, and environmental impact statements provide information on the history and operation of the wastewater reclamation facilities, such as flow rates and nutrient concentrations, and depth and performance of injection wells. Petty and Peterson (1979) summarized injection practices on Maui, focusing on small resort and condominium waste facilities ("package" treatment plants) with injection wells, which were numerous at the time.

Injection plume dimensions have been estimated for all three County wastewater reclamation facilities. Burnham and others (1977) modeled injection at the Kahului WWRF and

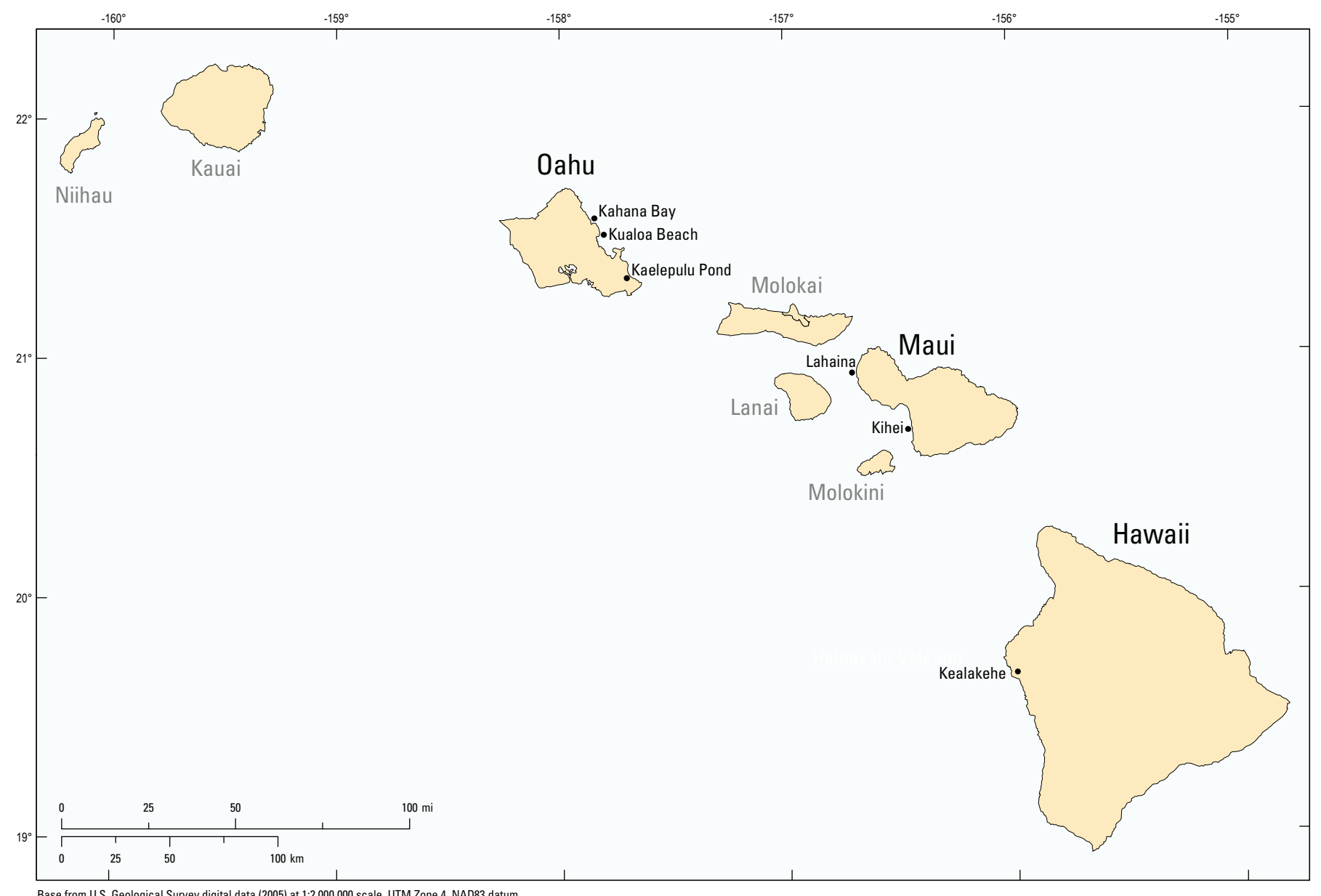

Figure 4. Map showing locations (black dots) of wastewater and nutrient source tracking field studies in Hawaii since 2006. 
estimated the effluent plume to be $2,000 \mathrm{ft}$ wide, extending $2,000 \mathrm{ft}$ seaward and 1,000 ft inland from injection wells. Tetra Tech (1993) also estimated a width of 2,000 ft for the Lahaina plume and effluent traveltimes of 15-20 days from wells to shore (a distance of $0.3 \mathrm{mi}$, or $0.5 \mathrm{~km}$ ). Hunt (2007) estimated plume width at Kihei to be $0.93 \mathrm{mi}(1.5 \mathrm{~km})$ at the shore, with effluent traveltimes of 5 years or less within the entire plume and as little as 1 year at the plume apex. Injection wells at Kihei are $0.75 \mathrm{mi}(1.2 \mathrm{~km})$ from shore.

Injected wastewater effluent is buoyant in coastal aquifers as a result of its low salinity and density, equivalent to about
1-2 percent seawater. Despite being injected deep into saltwater beneath the coastal brackish groundwater lens, effluent has a strong propensity to rise back to shallow depths as it spreads out areally (fig. 5). This buoyant behavior has been known locally and simulated in laboratory and computer models since at least the 1970s (Wheatcraft and others, 1976; Burnham and others, 1977; Heutmaker and others, 1977; Peterson and others, 1978). Hunt (2007) revisited the topic some 30 years later at Kihei, preparing color renderings intended to convey plume geometry and behavior to a broader audience of stakeholders than the 1970s work, which preceded widespread availability

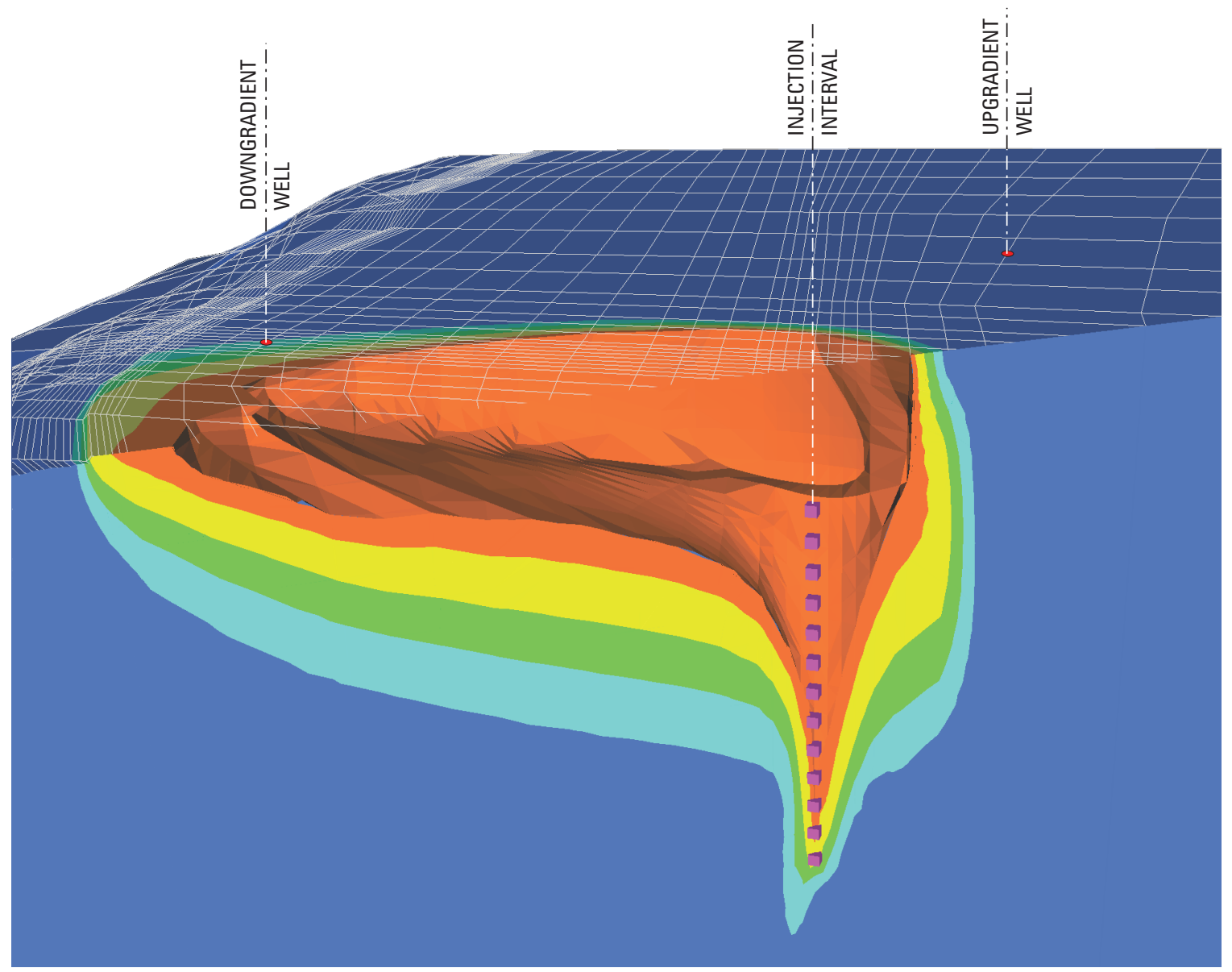

VERTICAL EXAGgERATION 20X

LOCATION AND PORTION

OF MODEL SHOWN

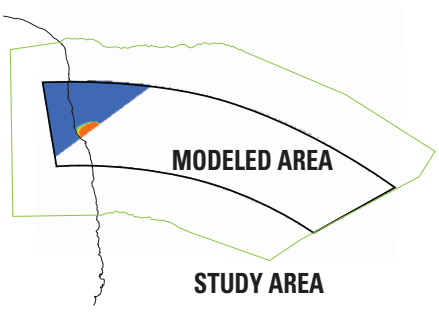

EFFLUENT-TRACER CONCENTRATION, IN PERCENT EFFLUENT

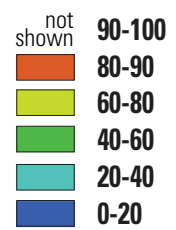

Pink cubes are fluid-source cells in the model corresponding to the injection interval (-47 to -194 feet below sea level)

Figure 5. Cutaway block diagram showing a modeled injection plume, Kihei, Hawaii (from Hunt, 2007). 
of computerized color graphics and was known and understood mostly among technical specialists.

Confining units within the aquifer system may impede buoyant rise of effluent, restricting it to greater depths in the aquifer and possibly forcing discharge farther offshore. Burnham and others (1977) described and simulated this effect at Kahului. Confinement apparently is less effective at Kihei and Lahaina, where effluent can be detected right at the shore, as will be shown later in this report.

Injection holds several advantages over other means of wastewater disposal, some of which were reasons for its adoption on Maui. First, injection wells are far less costly than deep-ocean outfalls, which are large-scale engineering and construction projects. Second, although injected effluent must emerge offshore, general concepts of groundwater flow suggest that discharge should be spread diffusely over a broad area, thereby allowing seawater dilution by waves and currents to lessen effects on the marine environment. Third, effluent flow through the aquifer is thought to allow some degree of dilution by ambient groundwater and the possibility of at least partial attenuation of constituents by sorption, bacterial consumption, compound breakdown, and other processes. The extent to which these idealizations hold true and lessen marine impact depends on factors such as the volume injected, constituent concentrations and mass loads, proximity to shore, geochemical and bacterial conditions in the aquifer, strength of ocean mixing, and aquifer heterogeneity, which cannot be known but which may include both confining units that would tend to be more protective and preferential flow pathways that would exacerbate focused, point-source discharge rather than the diffuse discharge generally envisioned.

Nuisance macroalgal blooms in the late 1980s and early 1990s prompted an intensive study of the Lahaina coast by USEPA, NOAA (National Oceanic and Atmospheric Administration), and teams of university researchers and environmental consultants. Elements of the land-sea margin that were studied included stream runoff, groundwater flow, the marine water column and benthic pore waters, and benthic biological communities. Results were summarized in the West Maui Watershed Owner's Manual (West Maui Watershed Management Advisory Committee, 1997).

Terrestrial nutrient loads and marine nutrient concentrations at Lahaina were studied by Tetra Tech (1993), Dollar and Andrews (1997), and Soicher and Peterson (1997). Principal sources of nutrients were identified as sugarcane and pineapple agriculture, municipal wastewater injection, cesspools, and resorts and golf courses. Runoff was described as episodic and only briefly active, in contrast to groundwater flow, which is a constant, year-round process. Soicher and Peterson (1997) pointed out that the Honokowai coastal area received not only effluent from the nearby Lahaina WWRF but also the greatest amounts of agricultural fertilizer nutrients along the coast through the regional groundwater. Hunt (2007) later estimated effluent and background nutrient loads in the Kihei area and provided evidence of natural attenuation of nitrogen and phosphorus in the anoxic effluent plume within the aquifer.
Marine studies in the 1970s and later documented strong inshore-offshore nutrient gradients across Lahaina and Kihei reefs, with high inshore nutrient concentrations attributed to emerging groundwater (for example, Dollar and Andrews, 1997). Laws and others (2004) noted that nutrient concentrations at many inshore sites exceeded State water-quality standards for open marine waters and embayments. Smith and others (2005) measured higher algal nitrogen concentrations and heavier algal nitrogen isotopic compositions inshore than offshore at Kahekili Beach north of Lahaina. They also reported low-salinity porewater (20 parts per thousand, or 57 percent seawater) at one site in $15 \mathrm{~m}$ water depth, which would place the site more than $100 \mathrm{~m}$ from shore. Smith and Smith (2007) reported similar inshore-offshore nutrient gradients in algae and water at Kihei and described algal growth experiments that led them to conclude that terrestrial nutrients promoted algal growth there. Street and others (2008) estimated submarine groundwater discharge and nutrient loads at sites around the Hawaiian Islands but did not report any link between their data at Honokowai Point and the nearby Lahaina WWRF.

Attempts at wastewater detection in the 1990s include an injected tracer test at the Lahaina injection wells using rhodamine fluorescent dye (Tetra Tech, 1994) and three related nutrient sampling efforts in the same vicinity (Tetra Tech, 1994; Bourke, 1996; Dollar and Andrews, 1997). All these surveys were inconclusive in that they failed to produce compelling indications of emerging effluent. However, surveys were concentrated near Honokowai Point, generally more than 100 $\mathrm{m}$ offshore, and in fairly deep water (mostly on the order of 10-30 m). In the Tetra Tech dye-tracer test, offshore fluorometer transects were conducted by boat across a $1.5 \times 1.5$ nauticalmile grid $(2.8 \times 2.8 \mathrm{~km})$ centered on Honokowai Point, directly offshore of the injection wells in the presumed direction of effluent flow. Results were judged to be inconclusive, with most fluorescence readings indistinguishable from background levels. Readings three times background were measured at two sites in the southeast corner of their grid, $300 \mathrm{~m}$ offshore and in $30 \mathrm{~m}$ of water. Tetra Tech (1994) mentioned that "Freshwater seeps and bubbles had been previously reported in this area, but much closer to the shore in very shallow water (less than $2 \mathrm{~m}$ ). Further investigation would be required in this area to confirm the presence of elevated tracer and effluent concentrations." It appears that these shallow bubbling seeps were not investigated thoroughly thereafter, although their description and location sound very much like the nearshore submarine springs sampled in our study that yielded positive detection of wastewater across several chemical and isotopic tracers.

In contrast to the introduced dye-tracer test, "inherent" wastewater tracers have also been used on Maui. Dollar and others (1999) and Atkinson and others (2003) analyzed for estrogen as an inherent wastewater tracer of cesspool and injection-well effluents at West Maui and at Maalaea Bay in South Maui (as well as at a number of other sites worldwide). Hunt (2007) modeled the Kihei injection plume and analyzed for inherent tracers in groundwater and treated effluent. He 
concluded that a well in the downgradient part of the modeled plume did indeed contain an effluent fraction as indicated by detection of pharmaceuticals, waste indicator compounds, heavy $\delta^{15} \mathrm{~N}$ in both nitrate and ammonium, and light $\delta^{11} \mathrm{~B}$ (from laundry detergents). Hunt reported $\delta^{15} \mathrm{~N}$ of +15 per mil, or parts per thousand, in effluent nitrate, +23 per mil in the plume well nitrate (enriched in the heavier isotope by denitrification of effluent nitrogen), and +5 per mil in upland groundwater nitrate outside the plume. Smith and Smith (2007) also measured $\delta^{15} \mathrm{~N}$ in benthic macroalgae along the Kihei coast; although they did not sample within Hunt's modeled plume, they did obtain heaviest $\delta^{15} \mathrm{~N}$ values ( +10 to +11 per mil) just north of it. Algae transects by Dailer and others (2008) did span the Kihei and Lahaina injection plumes and obtained $\delta^{15} \mathrm{~N}$ as heavy as +18 per mil at Kihei and +43 per mil at Lahaina. These algae $\delta^{15} \mathrm{~N}$ surveys constitute the earliest convincing detection of the effluent plumes offshore, preceding the present study and later being confirmed by our detection of additional supporting tracers other than $\delta^{15} \mathrm{~N}$ alone.

Basic information on nutrients, sewage plumes, and wastewater-related organic compounds is extensive in the scientific literature. A particularly well-studied sewage wastewater plume at Cape Cod, Massachusetts, has served as a research site for U.S. Geological Survey and other scientists for several decades (LeBlanc, 1984). Studies there have illuminated microbially mediated geochemical processes and nutrient transformations within the plume, such as denitrification (Smith and others, 1991; Smith and Duff, 1998; Smith and others, 2001); ammonium transport, sorption, and nitrification (Bohlke and others, 2006; Miller and Smith, 2009); and phosphorus transport and sorption (Walter and others, 1996). Locally in Hawaii, denitrification and biodegradation of organic matter were documented in field studies of injection wells by Oberdorfer and Peterson (1982).

The general geology and hydrology of Maui were established by early studies. Stearns and Macdonald (1942) mapped the geology of Maui, and Sherrod and others (2007) recently published an updated, computerized geologic map. Yamanaga and Huxel (1969) and Takasaki (1972) evaluated water resources in the Lahaina and Central Maui areas and showed that groundwater near the coast is brackish and not suitable for drinking. Souza (1981) provided a status update for groundwater in the Lahaina area that contained a nitrate map. The Kihei and Lahaina areas were included in water budgets by Engott and Vana (2007) and in the numerical groundwater model of Gingerich (2008).

\section{Acknowledgments}

The Hawaii State Department of Health $(\mathrm{HDOH})$ contributed funding, chose field case studies, and helped develop survey methods and strategy collaboratively, particularly during an early proof-of-concept phase. Thanks to Watson Okubo and staff at Clean Water Branch, including Josh Marvit, Scot Nishimoto, Jennifer Doi, Gerald Higuchi, Dale Mikami, and Scott Murakama.
Thanks also to U.S. Environmental Protection Agency (USEPA) Region IX for funding and encouraging program development, specifically to Wendy Wiltse and Janet Hashimoto.

Meghan Dailer (University of Hawaii) and Darla White (University of Hawaii and Hawaii Department of Land and Natural Resources, Division of Aquatic Resources) directed us to submarine springs at Kahekili Beach near Lahaina, conducted inverted-funnel sampling of the springs by scuba, and collected macroalgae samples. Dailer also collected algae at Kihei, dried and ground all samples, and forwarded sample powders to the USGS Stable-Isotope Laboratory for analysis. We are very grateful to Ms. Dailer and Ms. White for their generous contributions of time, effort, and expertise, and for lending a kayak for our surveys.

County of Maui Environmental Management Division assisted in sampling effluent at Lahaina WWRF in 2008 (thanks to Jake Kostrick, Greg Jensen, and others) and at Kihei WWRF in 2004 (thanks to Jerry Morgan, Clarence Solomon, Anita Fernandez, and others). Scott Rollins explained facets of system operations and provided treatment-plant records on several occasions. Steve Parabicoli explained the water recycling and reuse program and the different levels of effluent treatment and grades of reclaimed water, assisted with sampling arrangements, and assessed whether active cesspools still remain near Kalama Park at Kihei.

Jeff Perreault (USGS) collected an important groundwater end-member sample at the upland Puukolii well in the Lahaina area. John Engott (USGS) assisted with methods development and proof-of-concept surveys on Oahu in 2006-2007.

Author Hunt has benefited from discussions of marine chemistry and ecology with various colleagues. Watson Okubo at $\mathrm{HDOH}$ and Wendy Wiltse at USEPA clarified aspects of State and Federal monitoring programs and provided a wealth of historical knowledge and reports. Several colleagues presently or formerly at the University of Hawaii provided data, references, and patient explanations, including Celia Smith, Frank Sansone, Steven Dollar, Iuri Herzfeld, Jennifer Smith, Brian Popp, Craig Glenn, Meghan Dailer, Darla White, and Cynthia Hunter. Russell Sparks and Bob Nishimoto at Hawaii Department of Land and Natural Resources, Division of Aquatic Resources, also participated in helpful discussions.

\section{Survey Setting, Methods, and Results}

Nearshore marine surveys were conducted across the Kihei and Lahaina municipal wastewater injection plumes where they were expected to discharge at Kalama Park in South Kihei and at Kahekili-Honokowai Beach near Lahaina (hereafter simplified to "Kihei" and "Lahaina" after their respective Wastewater Reclamation Facilities). Both locales have shallow fringing reefs, with generally clear water and good visibility of the patchy sand and rock bottom (fig. 6 and fig. 7). A few submarine springs are prominent enough 


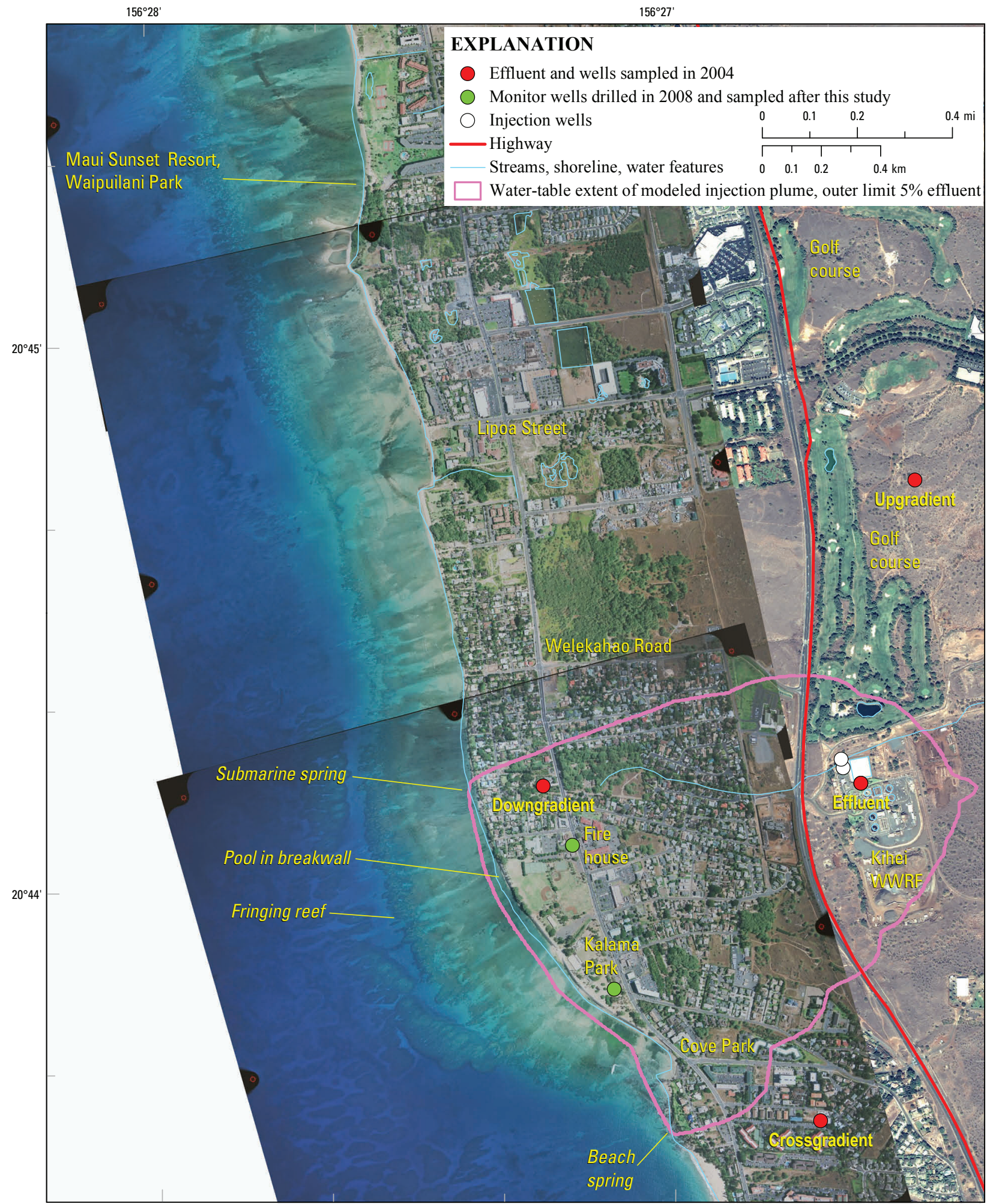

Base from U.S. Geological Survey digital data (2004) at 1:24,000 scale, UTM Zone 4, NAD83 datum. Digital orthohphotomosaic (lighter in tone) from DigitalGlobe (2006) overlain by individual frames from University of Hawaii Coastal Geology Group (2007), crudely rectified here.

Figure 6. Aerial photograph showing fringing reef in the Kalama Park area of Kihei, Hawaii. 


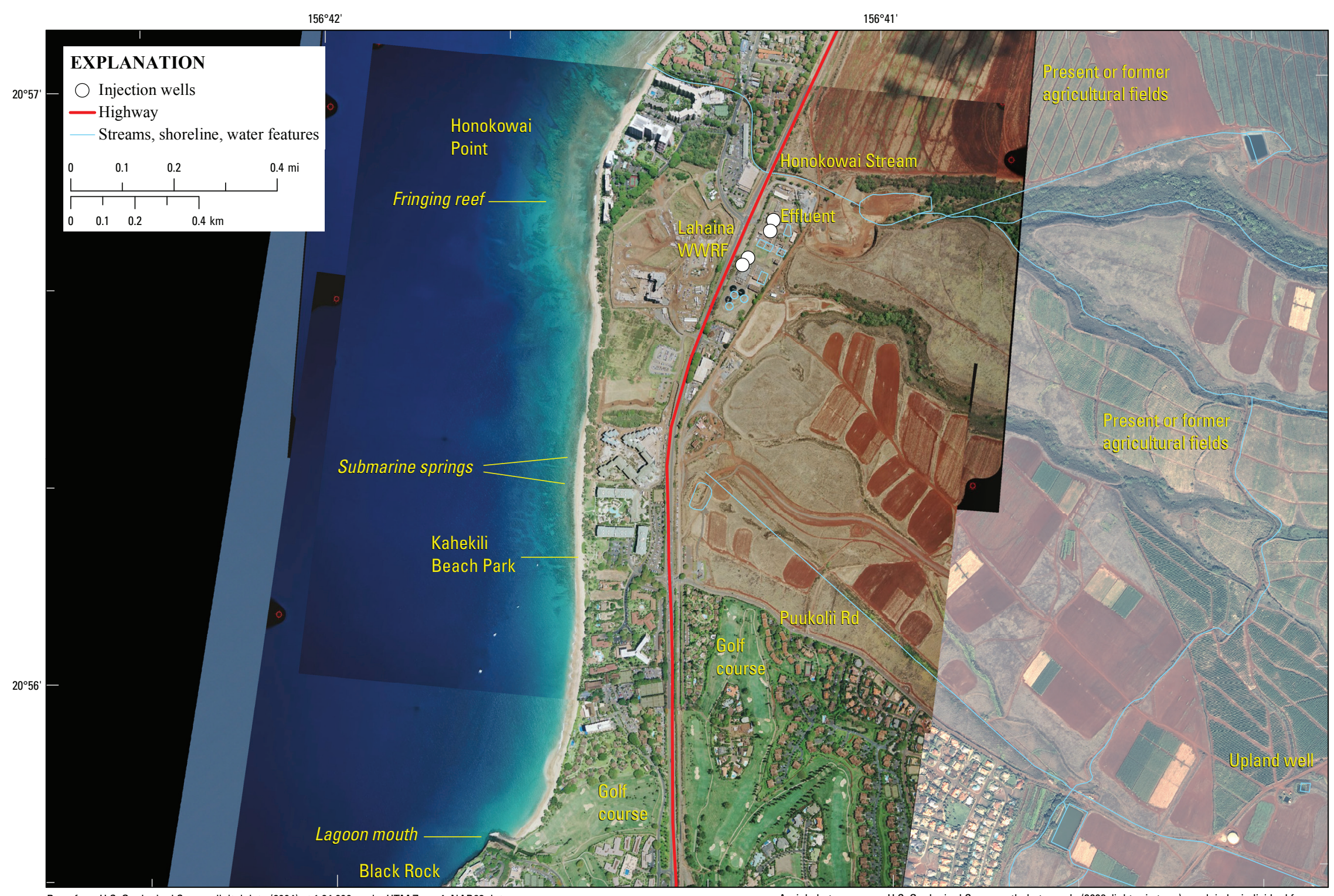

Base from U.S. Geological Survey digital data (2004) at 1:24,000 scale, UTM Zone 4, NAD83 datum. Aerial photograps are U.S. Geological Survey orthohptoquads (2006; lighter in tone) overlain by individual fr
from University of Hawaii Coastal Geology Group (2007; crudely rectified here) for better underwater detail.

Figure 7. Aerial photograph showing fringing reef from Kahekili Beach to Honokowai Point near Lahaina, Hawaii. 


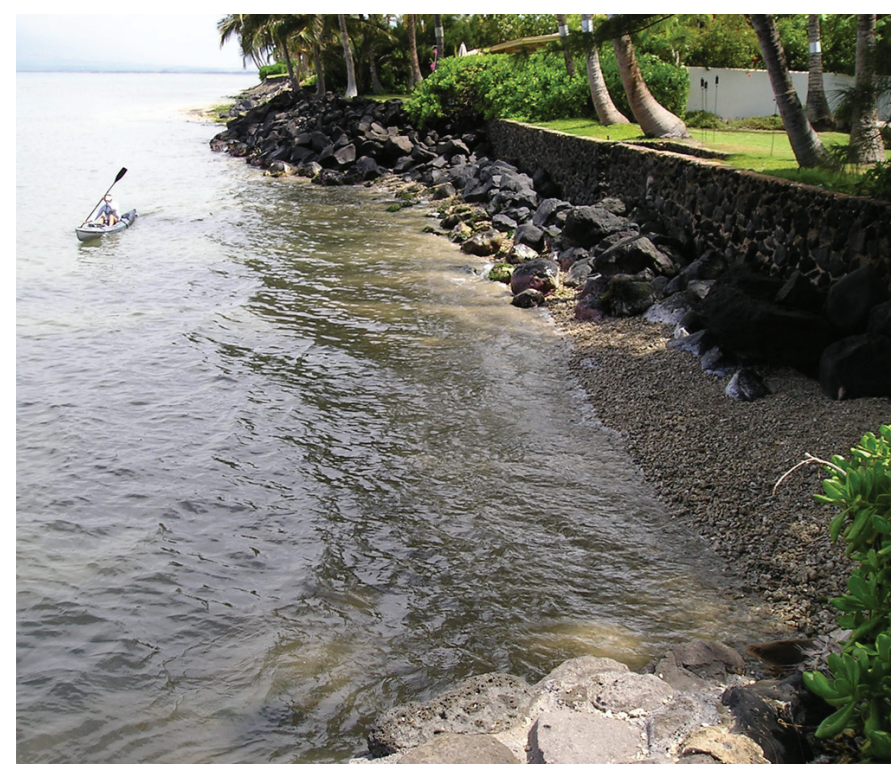

Figure 8. Photograph showing the seawalls and narrow beach along a kayak survey at Kihei, Hawaii (Photograph by Charles Hunt).

to be readily noticeable, but groundwater almost certainly discharges diffusely along the entire shore in addition and is simply masked readily by seawater mixing where it is not focused. Groundwater is recharged upslope (by rainfall, stream infiltration, and irrigation) and then flows to the shore, passing beneath agricultural and urban lands that may contribute additional nutrients.

At Kihei, a few areas of the fringing reef flat are intertidal, but it is mostly knee- to waist-deep for $100 \mathrm{~m}$ or so offshore at low tide. Sand beach is continuous in the north, but the south part of the area is armored by seawalls and rock revetments with small beaches of carbonate sand or gravel in a few places (fig. 8). There are several intermittent streams along the coast, but none were flowing during the surveys and all had sand berms blocking them, most with standing backwaters at about sea level.

The Kalama Park area was targeted for surveying because it lies downhill from the County injection wells and coincides with the modeled injection plume of Hunt (2007). Figure 9 portrays the plume as a series of colored bands at 20-percent effluent increments to an outer limit of 5 percent effluent; the orange band is $80-100$ percent effluent, with the upper 10 percent stripped off to allow a view "down the throat" of the plume. Although injection is deep into the volcanic aquifer ( -47 to -194 feet below sea level) at the upland facility, effluent flows upward through sediments beneath the lowland coastal plain before discharging. Overlain on the plume are marine water-column samples submitted for laboratory analysis in this study (appendix B shows detailed remarks for each site as well as maps of reconnaissance water samples and algae samples). Treated and disinfected wastewater effluent was sampled at the Kihei Wastewater Reclamation Facility in 2004 as were several nearby wells by Hunt (2007); these samples were subjected to exhaustive laboratory analyses comparable to our 2008 samples. New monitor wells were installed in 2008 but were not available in time for this study (however, they were sampled recently, in July 2009, to provide data for future research).

Peculiar biogenic mounds (bioherms) were noticed at two sites about $10 \mathrm{ft}$ from shore at Kalama Park but nowhere else in our surveys (fig. 9). They are elongate lobes about 3-5 ft long and not quite a foot high, light gray in color, and unlike other macroalgae in the area. They may be mounds of cyanobacteria (blue-green algae) similar to those at Shark Bay, Australia, that are thought to be modern equivalents of ancient stromatolites, or possibly polychaete (tubeworm) mounds that have been described on Hawaiian reef flats (Bailey-Brock, 1976). Also noted on figure 9 are a prominent submarine spring, a pool in the breakwall where discharging groundwater is partly shielded from seawater mixing, and a beach spring issuing in the intertidal zone from a volcanic-rock promontory. A second area was surveyed at Waipuilani Park near the Maui Sunset resort (more than a mile north of the plume; fig. 9) because of long-standing interest in causes of heavy algal accumulation there (Carroll, 2004; Smith and Smith, 2007).

At Lahaina (fig. 7), the fringing reef is intertidal at Honokowai Point but deepens southward along Kahekili Beach, so that one is in waist- to shoulder-deep water less than $5 \mathrm{~m}$ off the beach in many places. As at Kihei, injection is deep into the volcanic aquifer ( -55 to -229 feet below sea level) and effluent flows upward through coastal sediments beneath the narrow coastal plain before discharging at the coast. Prior indications that effluent might be discharging at Kahekili Beach include submarine springs with conspicuous gas bubbles and heavy algal $\delta^{15} \mathrm{~N}$ values measured by Dailer and others (2008). The springs are about $2 \mathrm{~m}$ deep and are not directly seaward of the Lahaina injection wells at Honokowai Point but instead lie much farther southwest than would be expected for effluent discharge. Sand beach is continuous from Honokowai Point to Black Rock, and the Kahekili beachface (fig. 10) is steep and the beach much wider than at Kihei.

Water samples for laboratory analysis were taken along the length of Kahekili Beach from Honokowai Point to Black Rock (fig. 11), at the Lahaina Wastewater Reclamation Facility (treated and disinfected wastewater effluent), and at an agricultural irrigation well in the uplands. Appendix $\mathrm{C}$ shows detailed remarks for each site, as well as maps of reconnaissance water samples and algae samples. Honokowai Stream, a major channelized and concrete-lined stream, enters the ocean north of Honokowai Point. It was not discharging during our surveys, but there was standing backwater behind its closed sand berm, offering the potential for slow seepage through the sand.

To the far south at Black Rock, brackish groundwater discharges from a channelized lagoon (fig. 12), in which a bright green phytoplankton bloom was apparent during our visits. The lagoon also conveys runoff during rainstorms, because the surrounding golf course is graded toward it, and it is analogous to other low-lying stream backwaters that intercept the shallow water table, except that it is not dammed by a sand berm 


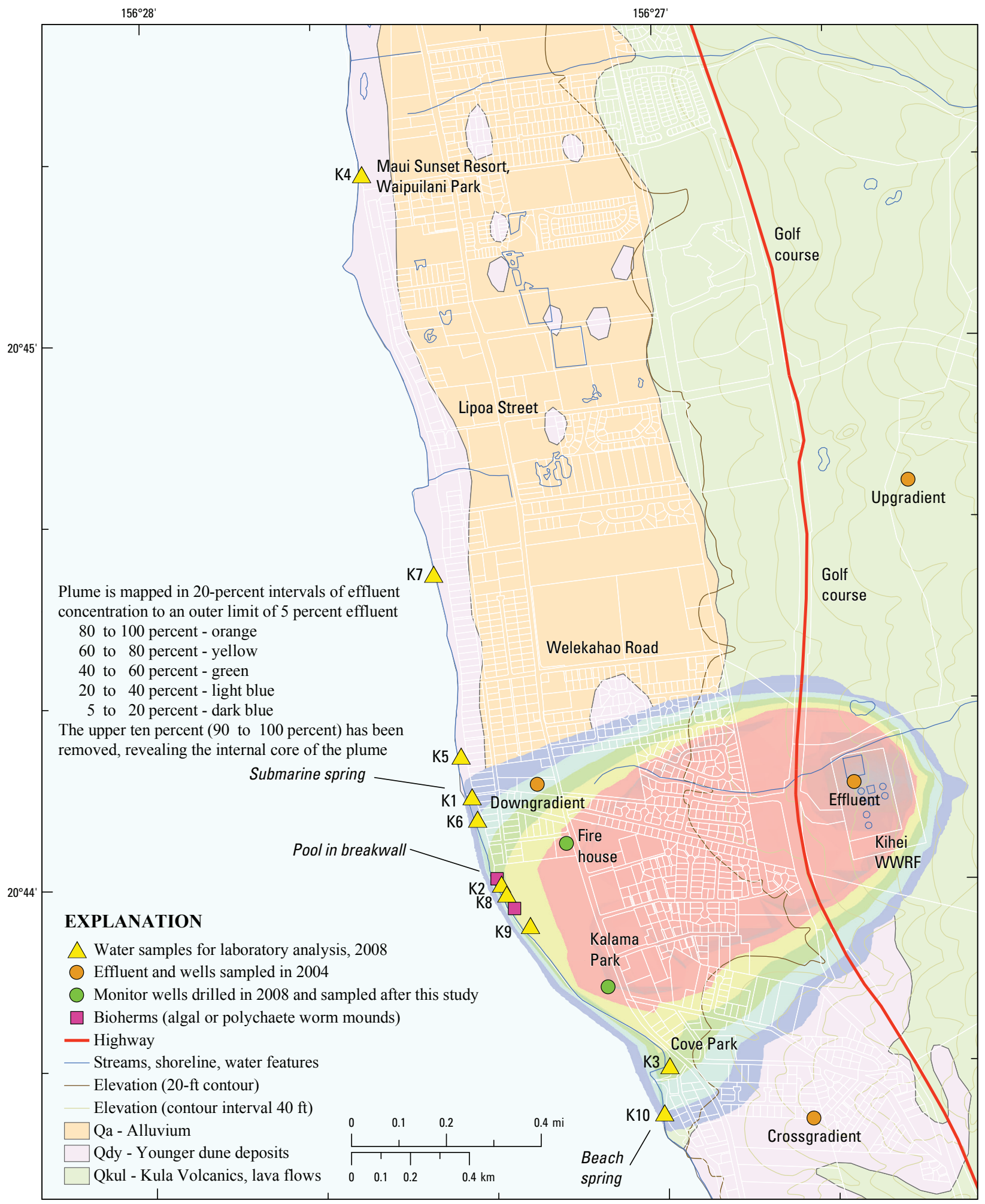

Base from U.S. Geological Survey digital data (2004) at 1:24,000 scale, UTM Zone 4, NAD83 datum. Street and property boundaries are from Hawaii State 0ffice of Planning tax maps (2008). Geology is from Stearns and Macdonald (1942) and Sherrod and others (2007).

Figure 9. Map showing locations of laboratory water samples at Kihei, Hawaii, with algal bioherms and the modeled injection plume of Hunt (2007). 


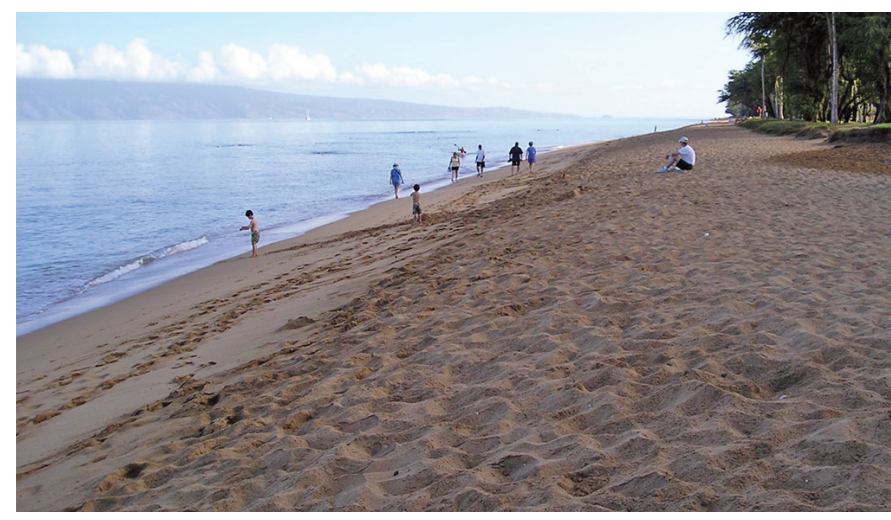

Figure 10. Photograph showing the broad, steep beach along a kayak survey at Kahekili Beach Park near Lahaina, Hawaii. Kayak is at left, at middle distance beyond people strolling (Photograph by Charles Hunt).

and instead is kept open perennially by a boulder breakwater. Dollar (1991) previously found that nutrient concentrations in this lagoon were highly elevated compared to adjacent marine waters.

Surveys were conducted during May 6-8 and May 20-23, 2008, during fair weather: sunny, with light winds, and with very small surf. Surveys were at low tide, as much as could be accomplished (tidal range in Hawaii is roughly $1 \mathrm{ft}$ at neap tide and 2-2.5 ft at spring tide). Multiple surveys were conducted at each locale:

1. trolling instrument surveys (wading and kayak) with a multiparameter water-quality sonde;

2. reconnaissance marine water-column samples for field measurements;

3. marine water-column samples for laboratory analysis, plus wastewater and groundwater wells;

4. and benthic algae samples for laboratory analysis.

Surveys were tiered by expense and complexity, progressing from least expensive instrument trolling and field measurements on quick-reconnaissance water samples to more expensive (but more diagnostic) laboratory analyses of water-column samples targeted from earlier reconnaissance. Benthic algae provided an added advantage of time-integrating their nutrient source (exposed continually, the algae incorporate nutrients with some average composition and may yield more stable data than an instantaneous sample, such as a grab sample from the water column). It was the nitrogen isotopic composition $\left(\delta^{15} \mathrm{~N}\right)$ of the algae that was of main interest in this study as a useful tracer of nitrogen origin.

Comprehensive results of the surveys are provided in maps and tables in appendixes $\mathrm{A}$ to $\mathrm{C}$ at the end of this report. Selected maps and tables are used in the main body of the report to discuss the significance of survey results. Table 1 lists laboratory analyses and field measurements that were made.

\section{Trolling Instrument Surveys}

Trolling instrument surveys provided continuous evaluation of water properties along transects, helping to guide later sampling by identifying anomalies such as brackish groundwater discharge ("hotspots," one might call them). Maps and tables of the instrumental data are included in appendix A. Surveys were made with a multiparameter water-quality sonde (Hydrolab DS-5) in recording mode, paired with a global positioning system (GPS) receiver recording position fixes every 30 seconds. Data files were merged later on common time stamps and loaded into a geographical information system (GIS) for mapping. Surveys were conducted by wading (with the sonde bracketed in an improvised floating platform; fig. 13) and by kayak (with the sonde suspended from the kayak stern; fig. 14). In both approaches, sensors were positioned just below water surface, in the top layer of the water column, to best detect any floating layer of fresher, lighter water that might accumulate there. Wading and kayak speeds were slow enough to allow sensors to equilibrate to stable readings.

Presurvey tasks entailed calibrating the various sensors and assigning the time interval and parameters to be recorded: temperature, salinity, specific conductance, $\mathrm{pH}$, turbidity, dissolved oxygen, dissolved-oxygen saturation, barometric pressure, chlorophyll-a (in both micrograms per liter and sensor voltage), and internal battery voltage. Standard solutions were used to calibrate specific conductance, $\mathrm{pH}$, turbidity, and dissolved oxygen. Barometric pressure was set to the latest sealevel-adjusted observation from the nearest National Weather Service station. Temperature was checked against reference thermometers before the field campaign. Only chlorophyll was uncalibrated and unchecked, and was simply operated at the manufacturer's factory-calibrated setting, owing to the difficulty and cost of compiling sufficient wet-bench chlorophyll determinations to establish a field calibration curve. As a result, the chlorophyll data can be used only in a relative sense: they should be internally consistent and comparable from point to point within a single day's survey, but without confidence as to absolute value or comparability from day to day across multiple surveys and locales.

Calibration, file setup, and data upload to computer were accomplished using the manufacturer's software. Data were exported to tab-delimited text files and then imported into spreadsheet software. The freshwater fraction for each data point was computed as Fresh_fraction $=(1-$ Salinity/35). This presumes pure freshwater as the fresh end member rather than actual groundwater in the vicinity; however, this is an acceptable simplification for comparative reconnaissance and it avoids the inconsistency that arises when actual end members differ from locale to locale.

Isolated chlorophyll readings of 2,000,000 $\mu \mathrm{g} / \mathrm{L}$ reflected an out-of-range condition and were deleted. They may have resulted from ambient-light or turbidity interference or insufficient sensor warm-up time. In a few instances they corresponded to high chlorophyll voltage readings, but in most cases they did not. Entire parameter columns were deleted 


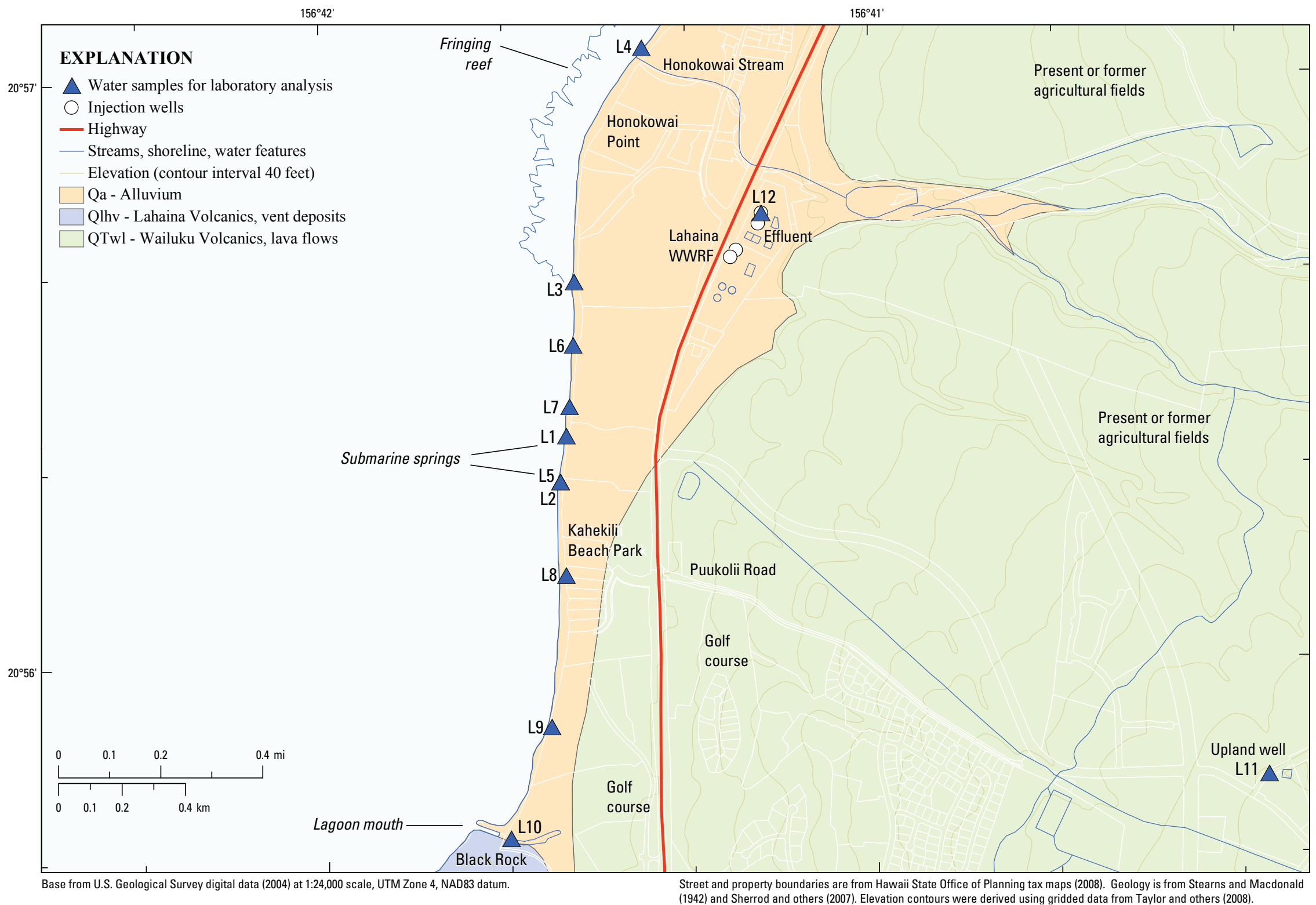

Figure 11. Map showing locations of laboratory water samples along Kahekili Beach near Lahaina, Hawaii. 
Table 1. Laboratory methods and field measurements for water and algae at Kihei and Lahaina, Hawaii, May 6-28, 2008.

[USGS, U.S. Geological Survey; N, nitrogen; P, phosphorus; H, hydrogen; O, oxygen; nm, nanometers; - , not applicable]

\begin{tabular}{|c|c|c|c|}
\hline Type of analysis or measurement & $\begin{array}{l}\text { USGS method } \\
\text { number }\end{array}$ & Laboratory or measurement & Reference \\
\hline Nitrogen, nitrite + nitrate, as $\mathrm{N}$ & Lab code 1979 & National Water Quality Laboratory ${ }^{1}$ & Fishman (1993) \\
\hline Nitrogen, ammonia, as $\mathrm{N}$ & Lab code 3116 & National Water Quality Laboratory & Fishman (1993) \\
\hline Orthophosphate, as $\mathrm{P}$ & Lab code 3118 & National Water Quality Laboratory & Fishman (1993) \\
\hline Nutrients + Total P and $\mathrm{N}$ & Schedule 2752 & National Water Quality Laboratory & $\begin{array}{l}\text { Fishman (1993); Patton and } \\
\quad \text { Kryskalla (2003) }\end{array}$ \\
\hline Pharmaceuticals (filtered at the laboratory) & Schedule 2080 & National Water Quality Laboratory & Furlong and others (2008) \\
\hline Waste indicator compounds, unfiltered & Schedule 4433 & National Water Quality Laboratory & Zaugg and others (2006) \\
\hline Stable isotopes of $\mathrm{H}, \mathrm{O}$ in water & Schedule 1142 & Reston Stable Isotope Laboratory ${ }^{2}$ & $\begin{array}{l}\text { Révész and Coplen } \\
\text { (2008a, 2008b) }\end{array}$ \\
\hline Stable isotopes of N, O in dissolved nitrate & Schedule 2900 & Reston Stable Isotope Laboratory & $\begin{array}{l}\text { Révész and Casciotti } \\
\quad(2007 \mathrm{a}, 2007 \mathrm{~b})\end{array}$ \\
\hline Stable isotopes of $\mathrm{N}$ in dissolved ammonium & Schedule 2898 & Reston Stable Isotope Laboratory & Hannon and Böhlke (2008) \\
\hline $\begin{array}{l}\text { Stable isotopes of } \mathrm{N} \text { and } \mathrm{N} \text {-concentration } \\
\text { in plant tissue (algae) }\end{array}$ & Schedule 2893 & Reston Stable Isotope Laboratory & Révész and Qi (2006) \\
\hline $\begin{array}{l}\text { Fabric brightener fluorescence at } 445 \mathrm{~nm} \\
\quad \text { emission wavelength }\end{array}$ & - & Meter reading on water samples & Turner Designs (2004) \\
\hline Specific conductance & - & Meter reading on water samples & $\begin{array}{l}\text { U.S. Geological Survey } \\
\text { (variously dated) }\end{array}$ \\
\hline $\begin{array}{l}\text { Specific conductance, salinity, } \mathrm{pH} \text {, dissolved } \\
\text { oxygen, turbidity, chlorophyll } a\end{array}$ & - & $\begin{array}{l}\text { Multiparameter water-quality sonde } \\
\text { (trolling surveys) }\end{array}$ & $\begin{array}{l}\text { U.S. Geological Survey } \\
\text { (variously dated) }\end{array}$ \\
\hline
\end{tabular}

${ }^{1}$ USGS National Water Quality Laboratory, Denver, Colorado

${ }^{2}$ USGS Reston Stable Isotope Laboratory, Reston, Virginia

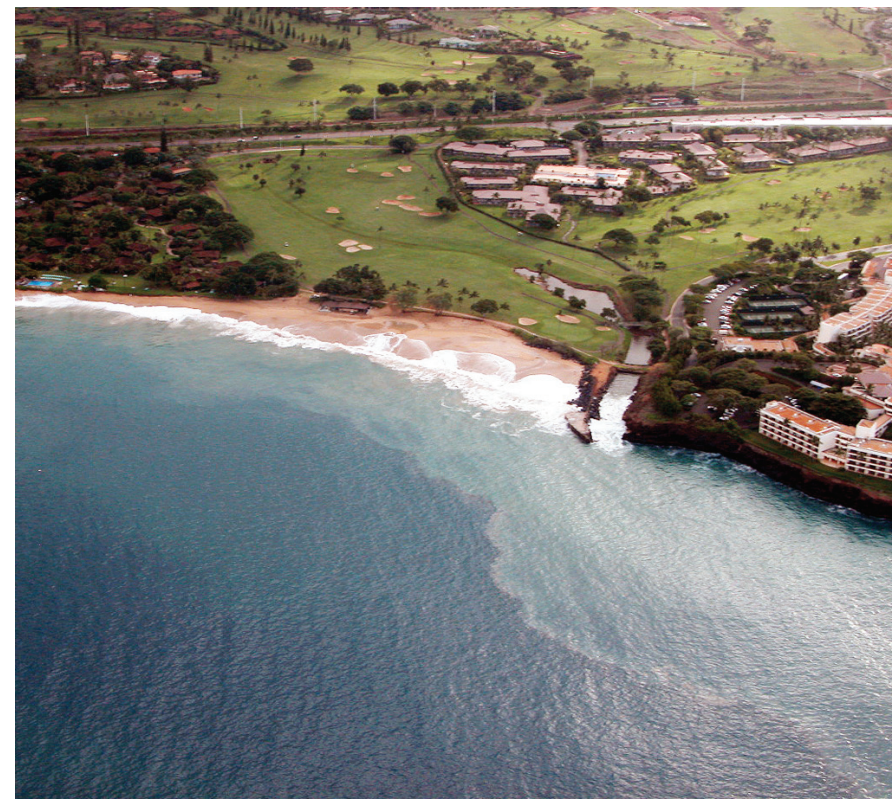

Figure 12. Oblique aerial photograph showing the channelized drainage lagoon at Black Rock near Lahaina, Hawaii (Photograph by University of Hawaii Coastal Geology Group).

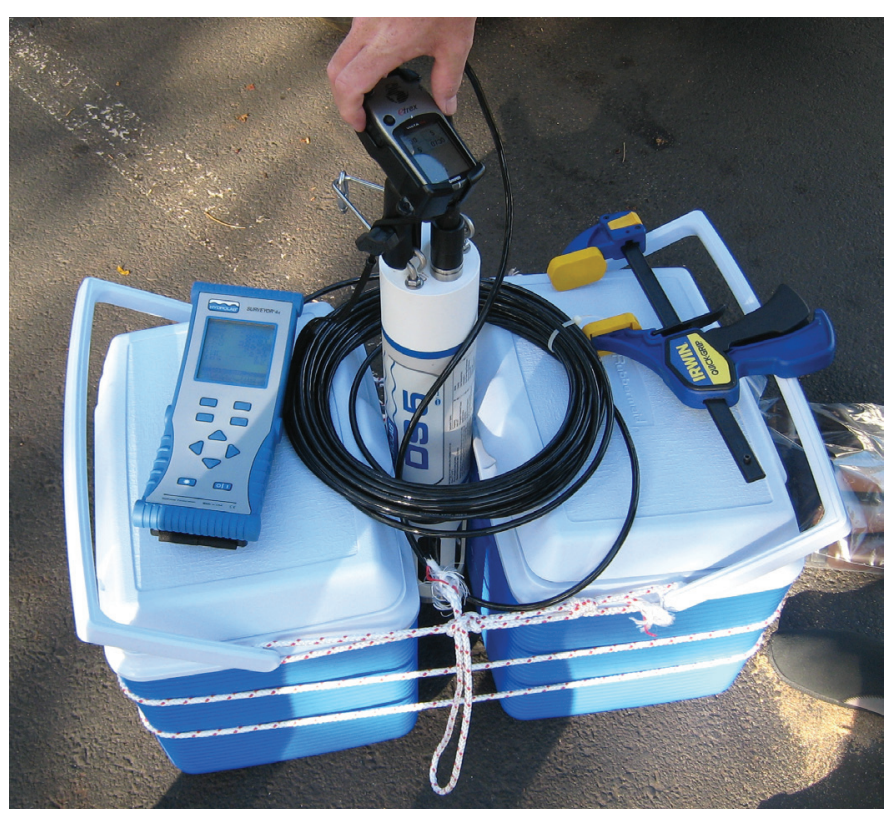

Figure 13. Photograph showing improvised wading platform for water-quality sonde (Photograph by Darla White). 


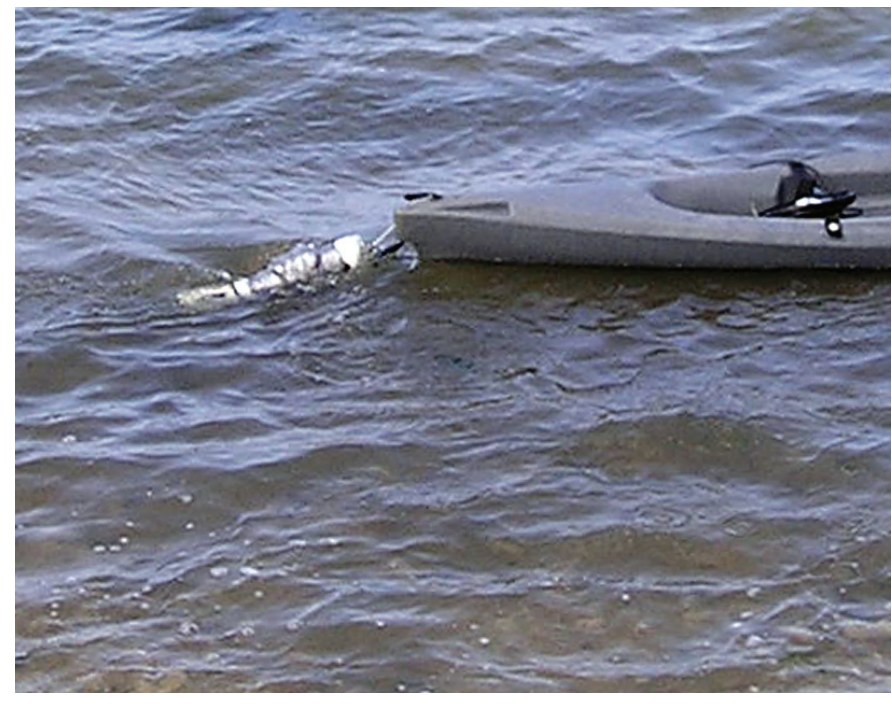

Figure 14. Photograph showing water-quality sonde tethered to kayak stern (Photograph by Charles Hunt).

because of faulty calibration (dissolved oxygen) or sonde set too shallow and planing out of the water so that sensors did not remain submerged (Lahaina kayak survey). Some remaining readings may not be generally representative of conditions but are difficult to second-guess: for example, high readings on turbidity and other sensors may reflect actual stirred-up sediment and cannot simply be ruled out as artifacts. Very low singlepoint salinities probably correspond with the sonde being lifted out of the water to avoid rocks or shallows; these were left in the data tables and censored at the mapping-analysis step.

\section{Reconnaissance Water-Column Samples}

Reconnaissance water samples also helped guide later laboratory water sampling. Roughly 20 samples were collected at each locale (18 at Kihei and 24 at Lahaina) and measured for specific conductance and fabric brightener fluorescence. Low conductance identified fresher waters, and high fluorescence indicated possible presence of optical brighteners added to laundry detergents. Results are listed in table 2, and sample sites and measurements are mapped in appendix B (Kihei) and appendix C (Lahaina). Sites with low conductance and high fluorescence were targeted for followup water sampling for laboratory analysis.

Marine water-column samples were collected in two ways. Most were collected by dipping just below the ocean surface (less than $10 \mathrm{~cm}$ ) to best collect from any floating layer of lighter, fresher water that might be present from groundwater discharging at the sea bottom and rising to the surface. Exceptions were Lahaina reconnaissance samples 11 and 24, which were collected using an inverted funnel to guide buoyant spring flow into the sample bottle (collected by Meghan Dailer and Darla White using scuba). The sample bottle was capped underwater once filled and then brought to shore. Samples were collected in $125-\mathrm{mL}$ amber polyethylene bottles prerinsed with sample water. These inverted-funnel samples succeeded in isolating emerging groundwater that was 24-percent fresh (sample 11) and 35-percent fresh (sample 24). A better approach for isolating the groundwater fraction would be porewater sampling with a piezometer where bottom sediments are soft enough.

\section{Laboratory Water-Column Samples}

Ten marine water-column samples per locale were collected for laboratory analysis of nutrients, stable isotopes, pharmaceuticals, and wastewater indicator compounds (table 3 to table 6), with maps of results in appendixes B and C). Lahaina samples L1, L2, and L5 were inverted-funnel samples from the ocean bottom at reconnaissance sites 11 (L1) and 24 (L2, L5). Samples L2 and L5 were sequential replicate samples at the same spring site; the second sample was taken in case the first didn't adequately capture the groundwater signature. Also sampled at Lahaina were the final treated and disinfected effluent at Lahaina WWRF and the water in an upland well (Puukolii well) to characterize wastewater and upland agricultural groundwater. Kihei effluent, upland groundwater, within-plume groundwater, and tap water were sampled in 2004 by Hunt (2007).

\section{Low-Level Detections}

A clarification is necessary for entries in tables 3 to 6 designated with "E" or "M," which might be referred to as "trace" by other laboratories. Many USGS methods use a combination of chromatography and mass-spectrometry for compound identification and quantitation. E-values are lower than the laboratory reporting level and lowest calibration standard used in the method. The concentration is flagged as "Estimated" because its numeric value is subject to more uncertainty than concentrations within the calibration range (bracketed by prepared calibration standards). M-flagged entries are lower still, so that no numeric concentration can be reported with sufficient confidence. However, in both cases the compound is known to be present by agreement between the chromatograph peak and mass spectrometer ionic fragments that match a given compound. For a more thorough discussion see Childress and others (1999). Also, ammonia analyses required dilution of the more saline samples, raising reporting levels for nondetections and introducing the apparent inconsistency of other detectable ammonium concentrations having values below stated reporting limits, a situation similar to the "estimated" category described above.

\section{Recomputation of Reported Nutrient Concentrations}

Dissolved nutrients (table 3 and table 4) determined from filtered water samples were nitrate+nitrite $\left(\mathrm{NO}_{3}+\mathrm{NO}_{2}\right)$, ammonium $\left(\mathrm{NH}_{4}\right)$, and orthophosphate $\left(\mathrm{PO}_{4}\right)$. Nitrogen 
Table 2. Field measurements on reconnaissance water samples collected at Kihei and Lahaina, Hawaii, May 7-8, 2008.

$[\mu \mathrm{S} / \mathrm{cm}$, microSiemens per centimeter; hr, hour; min, minute; sec, second; salinity is dimensionless and is computed from specific conductance using equation 9 from Wagner and others (2006); fluorescence was measured on a handheld fluorometer with optical brightener channel (excitation UV-375 nm, emission $445 \mathrm{~nm}$ ); negative values are simply low-level readings]

\begin{tabular}{|c|c|c|c|c|c|c|c|c|}
\hline $\begin{array}{c}\text { Site } \\
\text { number }\end{array}$ & $\begin{array}{c}\text { Fabric } \\
\text { brightener } \\
\text { fluorescence } \\
\text { (meter units) }\end{array}$ & $\begin{array}{c}\text { Specific } \\
\text { conductance } \\
(\mu \mathrm{S} / \mathrm{cm})\end{array}$ & Salinity & $\begin{array}{c}\text { Saltwater } \\
\text { fraction } \\
\text { (percent) }\end{array}$ & $\begin{array}{c}\text { Freshwater } \\
\text { fraction } \\
\text { (percent) }\end{array}$ & $\begin{array}{l}\text { Longitude } \\
\text { (degrees) }\end{array}$ & $\begin{array}{l}\text { Latitude } \\
\text { (degrees) }\end{array}$ & $\begin{array}{c}\text { Time } \\
\text { (hr:min:sec) }\end{array}$ \\
\hline \multicolumn{9}{|c|}{ Kihei, May 8, 2008} \\
\hline 1 & 0.317 & 47,500 & 30.9 & 88.3 & 11.7 & -156.450 & 20.727 & $8: 11: 27$ \\
\hline 2 & 0.490 & 45,700 & 29.6 & 84.6 & 15.4 & -156.450 & 20.728 & $8: 18: 42$ \\
\hline 3 & 0.226 & 49,300 & 32.2 & 92.0 & 8.0 & -156.451 & 20.729 & $8: 28: 45$ \\
\hline 4 & 0.900 & 50,100 & 32.8 & 93.7 & 6.3 & -156.452 & 20.729 & $8: 34: 29$ \\
\hline 5 & 1.270 & 50,200 & 32.9 & 94.0 & 6.0 & -156.454 & 20.731 & $8: 43: 20$ \\
\hline 6 & 0.912 & 50,800 & 33.3 & 95.1 & 4.9 & -156.455 & 20.732 & $8: 54: 45$ \\
\hline 7 & 1.322 & 46,900 & 30.5 & 87.1 & 12.9 & -156.455 & 20.733 & $9: 02: 21$ \\
\hline 8 & 1.586 & 42,900 & 27.6 & 78.9 & 21.1 & -156.455 & 20.733 & 9:07:19 \\
\hline 9 & 0.774 & 48,900 & 31.9 & 91.1 & 8.9 & -156.456 & 20.736 & $9: 20: 13$ \\
\hline 10 & 1.316 & 37,800 & 24.0 & 68.6 & 31.4 & -156.456 & 20.736 & $9: 23: 57$ \\
\hline 11 & 1.358 & 34,700 & 21.8 & 62.3 & 37.7 & -156.456 & 20.736 & $9: 26: 42$ \\
\hline 12 & 1.226 & 48,800 & 31.8 & 90.9 & 9.1 & -156.457 & 20.741 & 9:48:08 \\
\hline 13 & 0.902 & 40,000 & 25.5 & 72.9 & 27.1 & -156.457 & 20.743 & 9:55:19 \\
\hline 14 & 1.154 & 41,800 & 26.8 & 76.6 & 23.4 & -156.457 & 20.736 & $10: 41: 53$ \\
\hline 15 & 1.274 & 34,500 & 21.7 & 62.0 & 38.0 & -156.457 & 20.736 & $10: 45: 45$ \\
\hline 16 & 1.029 & 50,400 & 33.0 & 94.3 & 5.7 & -156.460 & 20.754 & 12:19:07 \\
\hline 17 & 2.011 & 45,000 & 29.1 & 83.1 & 16.9 & -156.460 & 20.754 & $12: 21: 37$ \\
\hline 18 & 1.840 & 45,800 & 29.7 & 84.9 & 15.1 & -156.460 & 20.756 & $12: 31: 07$ \\
\hline \multicolumn{9}{|c|}{ Lahaina, May 7, 2008} \\
\hline 1 & -0.166 & 52,400 & 34.5 & 98.6 & 1.4 & -156.691 & 20.951 & 9:12:44 \\
\hline 2 & 0.972 & 50,000 & 32.7 & 93.4 & 6.6 & -156.690 & 20.951 & 9:14:44 \\
\hline 3 & -0.078 & 52,200 & 34.3 & 98.0 & 2.0 & -156.692 & 20.949 & $9: 49: 29$ \\
\hline 4 & -0.094 & 52,200 & 34.3 & 98.0 & 2.0 & -156.692 & 20.946 & $10: 18: 46$ \\
\hline 5 & -0.178 & 52,200 & 34.3 & 98.0 & 2.0 & -156.692 & 20.945 & $10: 28: 00$ \\
\hline 6 & -0.255 & 52,100 & 34.3 & 98.0 & 2.0 & -156.692 & 20.944 & $10: 37: 27$ \\
\hline 7 & -0.302 & 52,000 & 34.2 & 97.7 & 2.3 & -156.693 & 20.942 & $10: 52: 25$ \\
\hline 8 & -0.253 & 52,300 & 34.4 & 98.3 & 1.7 & -156.693 & 20.942 & $10: 52: 56$ \\
\hline 9 & -0.338 & 52,200 & 34.3 & 98.0 & 2.0 & -156.693 & 20.941 & $11: 16: 49$ \\
\hline 10 & -0.328 & 52,300 & 34.4 & 98.3 & 1.7 & -156.693 & 20.940 & $11: 29: 37$ \\
\hline 11 & 3.206 & 41,400 & 26.5 & 75.7 & 24.3 & -156.693 & 20.940 & $11: 34: 18$ \\
\hline 12 & -0.212 & 51,400 & 33.8 & 96.6 & 3.4 & -156.693 & 20.939 & $11: 50: 30$ \\
\hline 13 & -0.116 & 51,400 & 33.8 & 96.6 & 3.4 & -156.693 & 20.938 & 12:03:01 \\
\hline 14 & -0.244 & 51,800 & 34.0 & 97.1 & 2.9 & -156.693 & 20.937 & $12: 06: 58$ \\
\hline 15 & -0.346 & 51,900 & 34.1 & 97.4 & 2.6 & -156.693 & 20.936 & $12: 11: 47$ \\
\hline 16 & -0.516 & 52,200 & 34.3 & 98.0 & 2.0 & -156.693 & 20.936 & $12: 16: 53$ \\
\hline 17 & -0.559 & 51,800 & 34.0 & 97.1 & 2.9 & -156.693 & 20.935 & $12: 28: 34$ \\
\hline 18 & -0.496 & 52,300 & 34.4 & 98.3 & 1.7 & -156.693 & 20.935 & $14: 21: 04$ \\
\hline 19 & -0.566 & 52,000 & 34.2 & 97.7 & 2.3 & -156.693 & 20.933 & $14: 25: 21$ \\
\hline 20 & -0.642 & 52,300 & 34.4 & 98.3 & 1.7 & -156.693 & 20.931 & $14: 29: 59$ \\
\hline 21 & -0.578 & 52,400 & 34.5 & 98.6 & 1.4 & -156.694 & 20.930 & $14: 35: 20$ \\
\hline 22 & -0.662 & 52,500 & 34.6 & 98.9 & 1.1 & -156.695 & 20.929 & $14: 41: 25$ \\
\hline 23 & 3.276 & 5,840 & 3.16 & 9.0 & 91.0 & -156.695 & 20.929 & $14: 44: 21$ \\
\hline 24 & 4.374 & 36,200 & 22.8 & 65.1 & 34.9 & -156.693 & 20.939 & $12: 00: 00$ \\
\hline
\end{tabular}


species were summed to dissolved inorganic nitrogen (DIN $=\mathrm{NO}_{3}+\mathrm{NO}_{2}+\mathrm{NH}_{4}$ ), and effluent was analyzed additionally for total dissolved nitrogen (TDN, including organic nitrogen) and total dissolved phosphorus (TDP). Results are tabulated in milligrams per liter $(\mathrm{mg} / \mathrm{L})$ as reported from the laboratory and recomputed to molar concentrations (micromoles per liter, $\mu \mathrm{M} / \mathrm{L}$, or simply $\mu \mathrm{M}$, for micromolar) for comparison to other studies using molar units. Nitrogen-tophosphorus molar ratios also were computed for comparison to the ecologically important Redfield ratios for assimilation (uptake) of nutrients by phytoplankton.

\section{Seawater Unmixing Calculation to Estimate Terrestrial Source Concentration in the Fresh Fraction}

Also listed in table 3 and table 4 are "source" nutrient concentrations, intended to undo the widely varying seawater content in marine-water samples and allow valid nutrient comparisons from sample to sample. Nutrients measured in the study are overwhelmingly terrestrial (land-derived), and local seawater contains almost no nutrients in comparison. Terrestrial waters (groundwater, wastewater effluent) can be compared straightforwardly, but nutrient concentrations in marine-water samples varied strongly and inversely with salinity, because seawater dilutes and reduces terrestrial nutrient concentrations.

To compensate, an "unmixing" calculation was applied to undo the seawater dilution each sample has undergone and estimate its terrestrial "source" concentration. The source concentration is the hypothetical concentration in the fresh fraction of the sample, and it can be compared to a terrestrial parent water from which the fresh fraction is suspected to have originated. Agreement is expected if the marine concentration results from simple seawater dilution of the parent. Disagreement could indicate loss or addition of the nutrient, wrong choice of parent water, errors or imprecision in analysis, or other factors beyond the simple set of assumptions proposed.

The equation used to accomplish seawater unmixing was:

$$
C_{1}=C_{\text {mix }}+\left(C_{\text {mix }}-C_{2}\right) \times\left(S_{\text {mix }}-S_{1}\right) /\left(S_{2}-S_{\text {mix }}\right)
$$

where:

$C_{1}$ is the concentration in component 1 , the hypothetical "source" concentration;

$C_{2}$ is the concentration in component 2 , in this case seawater;

$C_{\text {mix }}$ is the concentration in the mixed sample being evaluated;

$S_{1}$ is the salinity of component 1 , set equal to the suspected parent water (such as effluent or upland groundwater);

$S_{2}$ is the salinity of component 2 , in this case seawater and typically near 35 ; and

$S_{\text {mix }}$ is the salinity of the mixed sample being evaluated.
The equation simply projects the measured concentration of a sample back to what it would be if the sample was as fresh as the suspected parent water. The parent nutrient concentration is not used — only its salinity — so the computed source concentration is independent and can differ from the proposed parent (the source concentration is derived solely by projecting the sample concentration back along the salinity scale). The equation will work for any conservative solute, but it was used here to estimate the hypothetical nutrient concentration in a sample's terrestrial fraction. Seawater end-member concentrations for salinity and nutrients were taken from Dollar and Andrews (1997), for a reference seawater at Lahaina (average of samples $1 \mathrm{~m}$ and $2 \mathrm{~m}$ off the sea bottom at $20-\mathrm{m}$ water depth):

$$
\begin{aligned}
& S_{2}=34.93 \text { salinity } \\
& C_{2}=0.19 \mu \mathrm{M} \mathrm{NH}_{4} \\
& C_{2}=0.13 \mu \mathrm{M} \mathrm{NO}_{3}+\mathrm{NO}_{2} \\
& C_{2}=0.32 \mu \mathrm{M} \mathrm{DIN} \\
& C_{2}=0.12 \mu \mathrm{M} \mathrm{PO}_{4}
\end{aligned}
$$

Substituting these values and using DIN as an example, the equation becomes:

$$
\begin{aligned}
& \mathrm{DIN}_{\text {source }}=\mathrm{DIN}_{\text {mix }}+\left(\mathrm{DIN}_{\text {mix }}-0.32\right) \\
& \times\left(S_{\text {mix }}-S_{1}\right) /\left(34.93-S_{\text {mix }}\right)
\end{aligned}
$$

where:

$\mathrm{DIN}_{\text {source }}$ is the source concentration of dissolved inorganic nitrogen;

DIN $_{\text {mix }}$ is the measured DIN concentration in the sample being evaluated; and

remaining terms are as defined above.

Results of the calculations were reasonable and internally self-consistent (table 3 and table 4). Adjustments from measured nutrient concentrations to source concentrations were minimal for samples low in salinity and increased as sample salinity increased. Calculated source concentrations were roughly double the measured values for samples that were about half seawater and tens of times greater for saltier samples that were only a few percent fresh. Also reasonable is that some source concentrations were close to concentrations in proposed effluent and upland-water parents, consistent with simple seawater dilution of those parents (other samples had much lower source concentrations than parents, suggesting nutrient loss as well as dilution).

The choice of parent water did not affect results greatly, because possible parents were similarly fresh and the adjustment was influenced much more by the high salinity of marine samples than by small salinity differences between parents. At Kihei, samples outside the modeled plume were adjusted to parent salinity at nearby wells (upgradient well for samples $\mathrm{K} 4$, K5, and K7; crossgradient well for sample K10), and remaining samples were adjusted to effluent salinity, because they lie within the modeled plume and because overall chemistry indicates their fresh fraction is derived mainly from 
Table 3. Nutrients, stable isotopes, and physical parameters for water samples collected at Kihei, Hawaii, May 20, 2008, and November 15-17, 2004. $[<$, not detected at the reporting level shown; - , not analyzed or not applicable; E, estimated; $\mathrm{mg} / \mathrm{L}$, milligrams per liter; $\mu \mathrm{M}$, micromoles per liter; $\delta$, delta notation for isotopic compositions; per mil, parts per thousand; $\mu \mathrm{S} / \mathrm{cm}$, microSiemens per centimeter; ${ }^{\circ} \mathrm{C}$, degrees Celsius; UV, ultraviolet; nm, nanometers; >, greater than; mm Hg, millimeters mercury; Pcode, U.S. Geological Survey parameter code; salinity is computed from specific conductance using equation 9 from Wagner and others (2006); fluorescence was measured on a handheld fluorometer with optical brightener channel (emission wavelength $445 \mathrm{~nm}$ ) and rhodamine WT channel (emission wavelength $>570 \mathrm{~nm}$ ); terrestrial source concentration is an estimate of the terrestrial (effluent or groundwater) contribution to a sample, computed by unmixing the seawater dilution it has undergone using Equation 1, this report; 2004 samples are from Hunt (2007)]

\begin{tabular}{|c|c|c|c|c|c|c|c|}
\hline \multirow{6}{*}{ Constituent and category } & \multirow[b]{2}{*}{ Sample designation: } & \multirow[b]{2}{*}{ K1 } & \multirow[b]{2}{*}{ K2 } & \multirow[b]{2}{*}{ K3 } & \multicolumn{3}{|c|}{ Kihei nearshore marine and spring waters (2008) } \\
\hline & & & & & K4 & K5 & K6 \\
\hline & Type of sample: & $\begin{array}{l}\text { Submarine } \\
\text { spring/water } \\
\text { column }\end{array}$ & $\begin{array}{c}\text { Submarine } \\
\text { spring/water } \\
\text { column }\end{array}$ & Water column & Water column & Water column & Water column \\
\hline & Date: & 20080520 & 20080520 & 20080520 & 20080520 & 20080520 & 20080520 \\
\hline & Time: & 0850 & 1040 & 1120 & 1220 & 0910 & 0925 \\
\hline & Station ID: & 204350156271001 & 204350156271002 & 204350156271003 & 204350156271004 & 204350156271005 & 204350156271006 \\
\hline Nutrients, dissolved, mass concentration (as reported by lab) & Units & & & & & & \\
\hline Ammonium $\left(\mathrm{NH}_{4}\right)$, as $\mathrm{N}$ & $\mathrm{mg} / \mathrm{L}$ & 0.083 & 0.156 & $<0.200$ & $<0.200$ & $<0.200$ & $<0.200$ \\
\hline Nitrate plus nitrite $\left(\mathrm{NO}_{3}+\mathrm{NO}_{2}\right)$, as $\mathrm{N}$ & $\mathrm{mg} / \mathrm{L}$ & 1.95 & 2.93 & 1.85 & 0.025 & 0.171 & 0.429 \\
\hline Nitrite $\left(\mathrm{NO}_{2}\right)$, as $\mathrm{N}$ & $\mathrm{mg} / \mathrm{L}$ & - & - & - & - & - & - \\
\hline Dissolved inorganic nitrogen ( $\mathrm{DIN}=\mathrm{NO}_{2}+\mathrm{NO}_{3}+\mathrm{NH}_{4}$ ), as $\mathrm{N}$ & $\mathrm{mg} / \mathrm{L}$ & 2.03 & 3.09 & 1.85 & 0.03 & 0.17 & 0.43 \\
\hline Total dissolved nitrogen $(\mathrm{TDN}=\mathrm{DIN}+$ organic $-\mathrm{N})$, as $\mathrm{N}$ & $\mathrm{mg} / \mathrm{L}$ & - & - & - & - & - & - \\
\hline Orthophosphate $\left(\mathrm{PO}_{4}\right)$, as $\mathrm{P}$ & $\mathrm{mg} / \mathrm{L}$ & 0.112 & 0.210 & 0.077 & 0.011 & 0.013 & 0.022 \\
\hline Total dissolved phosphorus (TDP), as P & $\mathrm{mg} / \mathrm{L}$ & - & - & - & - & - & - \\
\hline Dissolved organic carbon (DOC) & $\mathrm{mg} / \mathrm{L}$ & - & - & - & - & - & - \\
\hline \multicolumn{8}{|l|}{ Nutrients, dissolved, molar concentration (computed) } \\
\hline Ammonium $\left(\mathrm{NH}_{4}\right)$, as $\mathrm{N}$ & $\mu \mathrm{M}$ & 5.9 & 11.1 & $<14$ & $<14$ & $<14$ & $<14$ \\
\hline Nitrate plus nitrite $\left(\mathrm{NO}_{3}+\mathrm{NO}_{2}\right)$, as $\mathrm{N}$ & $\mu \mathrm{M}$ & 139 & 209 & 132 & 1.8 & 12.2 & 30.6 \\
\hline Nitrite $\left(\mathrm{NO}_{2}\right)$, as $\mathrm{N}$ & $\mu \mathrm{M}$ & - & - & - & - & - & - \\
\hline Dissolved inorganic nitrogen (DIN), as $\mathrm{N}$ & $\mu \mathrm{M}$ & 145 & 220 & 132 & 1.8 & 12.2 & 30.6 \\
\hline Total dissolved nitrogen (TDN), as $\mathrm{N}$ & $\mu \mathrm{M}$ & - & - & - & - & - & - \\
\hline Organic nitrogen, by subtraction (organic-N=TDN-DIN), as $\mathrm{N}$ & $\mu \mathrm{M}$ & - & - & - & - & - & - \\
\hline Orthophosphate $\left(\mathrm{PO}_{4}\right)$, as $\mathrm{P}$ & $\mu \mathrm{M}$ & 3.62 & 6.78 & 2.49 & 0.36 & 0.42 & 0.71 \\
\hline Total dissolved phosphorus (TDP), as P & $\mu \mathrm{M}$ & - & - & - & - & - & - \\
\hline Dissolved organic carbon (DOC) & $\mu \mathrm{M}$ & - & - & - & - & - & - \\
\hline $\mathrm{N}: \mathrm{P}$ molar ratio $\left(\mathrm{DIN} / \mathrm{PO}_{4}\right)$ & - & 40 & 32 & 53 & 5 & 29 & 43 \\
\hline \multicolumn{8}{|l|}{ Terrestrial source concentration, seawater dilution removed } \\
\hline Source ammonium $\left(\mathrm{NH}_{4}\right)$, as $\mathrm{N}$ & $\mu \mathrm{M}$ & 11 & 24 & - & - & - & - \\
\hline Source nitrate plus nitrite $\left(\mathrm{NO}_{3}+\mathrm{NO}_{2}\right)$, as $\mathrm{N}$ & $\mu \mathrm{M}$ & 263 & 446 & 427 & 13 & 101 & 208 \\
\hline Source dissolved inorganic nitrogen (DIN), as N & $\mu \mathrm{M}$ & 274 & 470 & 427 & 13 & 101 & 208 \\
\hline Source orthophosphate $\left(\mathrm{PO}_{4}\right)$, as $\mathrm{P}$ & $\mu \mathrm{M}$ & 6.7 & 14.3 & 7.8 & 2.1 & 2.7 & 4.2 \\
\hline Terrestrial parent water used for seawater dilution adjustment & - & K-effluent & K-effluent & K-effluent & K-upgrad & K-upgrad & K-effluent \\
\hline \multicolumn{8}{|l|}{ Stable isotopes } \\
\hline$\delta^{18} \mathrm{O}$ (Oxygen-18/Oxygen-16 ratio) in water & per mil & -2.82 & -1.65 & -1.11 & -0.32 & -0.39 & -0.37 \\
\hline$\delta^{2} \mathrm{H}$ (Deuterium/Protium ratio) in water & per mil & -15.80 & -6.20 & -4.90 & -1.80 & 0.10 & -1.60 \\
\hline$\delta^{15} \mathrm{~N}^{-\mathrm{NO}_{3}}$ (Nitrogen-15/Nitrogen-14 ratio) in nitrate fraction & per mil & 14.38 & 16.53 & 14.17 & 5.99 & 10.73 & 16.56 \\
\hline${ }^{18} \mathrm{O}_{-} \mathrm{NO}_{3}$ (Oxygen-18/Oxygen-16 ratio) in nitrate fraction & per mil & 7.04 & 7.79 & 6.21 & 9.72 & 6.21 & 8.31 \\
\hline$\delta^{15} \mathrm{~N}^{-\mathrm{NH}_{4}}$ (Nitrogen-15/Nitrogen-14 ratio) in ammonium fraction & per mil & - & - & - & - & - & - \\
\hline \multicolumn{8}{|l|}{ Physical parameters, field measurements } \\
\hline Specific conductance & $\mu \mathrm{S} / \mathrm{cm}$ at $25^{\circ} \mathrm{C}$ & 27,200 & 30,300 & 38,300 & 47,400 & 47,500 & 46,100 \\
\hline Salinity (dimensionless), computed from specific conductance & - & 16.7 & 18.8 & 24.3 & 30.8 & 30.9 & 29.9 \\
\hline Seawater fraction, computed from salinity & percent & 47.7 & 53.7 & 69.4 & 88.0 & 88.3 & 85.4 \\
\hline Freshwater fraction, computed from salinity & percent & 52.3 & 46.3 & 30.6 & 12.0 & 11.7 & 14.6 \\
\hline Fabric brightener UV fluorescence at $445 \mathrm{~nm}$, meter reading & meter units & 2.221 & 3.462 & 1.466 & 1.634 & 0.750 & 0.945 \\
\hline Rhodamine WT fluorescence at $>570 \mathrm{~nm}$, meter reading & meter units & 0.161 & 0.107 & 0.184 & 0.360 & 0.171 & 0.119 \\
\hline $\mathrm{pH}$, field & $\mathrm{pH}$ units & - & - & - & - & - & - \\
\hline Temperature & ${ }^{\circ} \mathrm{C}$ & - & - & - & - & - & - \\
\hline Barometric pressure & $\mathrm{mm} \mathrm{Hg}$ & - & - & - & - & - & - \\
\hline Dissolved oxygen (meter) & $\mathrm{mg} / \mathrm{L}$ & - & - & - & - & - & - \\
\hline Dissolved oxygen (laboratory analysis) & $\mathrm{mg} / \mathrm{L}$ & - & - & - & - & - & - \\
\hline
\end{tabular}




\begin{tabular}{|c|c|c|c|c|c|c|c|c|c|c|}
\hline & & & & \multicolumn{6}{|c|}{ Kihei wastewater effluent, groundwater, and tap water (2004) } & \multirow[b]{6}{*}{ Pcode } \\
\hline K7 & K8 & К9 & K10 & K-effluent & K-downgrad & K-downgrad & K-crossgrad & K-upgrad & K-tap & \\
\hline Water column & Water column & Water column & Beach spring & $\begin{array}{l}\text { Kihei WWRF } \\
\text { treated effluent }\end{array}$ & $\begin{array}{c}\text { Down-gradient } \\
\text { well }\end{array}$ & $\begin{array}{l}\text { Effluent fraction with } \\
25 \% \text { upland-water } \\
\text { dilution removed }\end{array}$ & $\begin{array}{l}\text { Cross-gradient } \\
\text { well }\end{array}$ & $\begin{array}{l}\text { Upgradient } \\
\text { well }\end{array}$ & Tap water & \\
\hline 20080520 & 20080520 & 20080520 & 20080520 & 20041117 & 20041115 & - & 20041115 & 20041116 & 20041117 & \\
\hline 1005 & 1100 & 1155 & 1145 & 1100 & 1100 & - & 1500 & 1100 & 1000 & \\
\hline 204350156271007 & 204350156271008 & 204350156271009 & 204350156271010 & 204411156263800 & 204423156272501 & - & 204334156264301 & 204456156264101 & 204411156263801 & \\
\hline$<0.200$ & 0.109 & $<0.200$ & E0.011 & 0.26 & 0.09 & - & $<0.04$ & $<0.04$ & $<0.04$ & P00608 \\
\hline 0.352 & 3.77 & 0.056 & 1.30 & 6.32 & 3.21 & - & 1.54 & 1.11 & 0.58 & P00631 \\
\hline - & - & - & - & 0.101 & 0.008 & - & $<0.008$ & $<0.008$ & $<0.008$ & P00613 \\
\hline 0.35 & 3.88 & 0.06 & 1.31 & 6.58 & 3.30 & - & 1.54 & 1.11 & 0.58 & - \\
\hline - & - & - & - & 7.33 & 3.49 & - & 1.54 & 1.17 & 0.60 & P62854 \\
\hline 0.022 & 0.245 & 0.014 & 0.071 & 1.70 & 0.186 & - & 0.051 & 0.058 & 0.096 & P00671 \\
\hline- & - & - & - & 1.72 & 0.191 & - & 0.059 & 0.067 & 0.106 & P00666 \\
\hline - & - & - & - & 3.9 & 1.3 & - & $<0.3$ & E0.2 & - & P00681 \\
\hline$<14$ & 7.78 & $<14$ & E0.8 & 19 & 6.4 & 8.5 & $<2.9$ & $<2.9$ & $<2.9$ & P00608 \\
\hline 25.1 & 269 & 4.0 & 92.8 & 451 & 229 & 279 & 110 & 79.2 & 41.4 & P00631 \\
\hline- & - & - & - & 7.21 & 0.6 & 0.8 & $<.6$ & $<.6$ & $<.6$ & P00613 \\
\hline 25.1 & 277 & 4.0 & 93.6 & 470 & 235 & 287 & 110 & 79.2 & 41.4 & - \\
\hline- & - & - & - & 523 & 249 & 304 & 110 & 84 & 43 & P62854 \\
\hline- & - & - & - & 53 & 14 & 17 & 0 & 4 & 1 & - \\
\hline 0.71 & 7.91 & 0.45 & 2.29 & 54.9 & 6.01 & 7.4 & 1.65 & 1.87 & 3.1 & P00671 \\
\hline- & - & - & - & 55.5 & 6.17 & 7.5 & 1.90 & 2.16 & 3.4 & P00666 \\
\hline - & - & - & - & 325 & 108 & 139 & $<25$ & E17 & - & P00681 \\
\hline 35 & 35 & 9 & 41 & 9 & 39 & 39 & 67 & 42 & 13 & - \\
\hline - & 15 & - & E0.8 & 19 & 6.6 & 8.8 & - & - & - & - \\
\hline 92 & 526 & 78 & 96 & 451 & 237 & 290 & 110 & 79.2 & - & - \\
\hline 92 & 541 & 78 & 97 & 470 & 244 & 299 & 110 & 79.2 & - & - \\
\hline 2.3 & 15.3 & 7.1 & 2.3 & 54.9 & 6.21 & 7.66 & 1.65 & 1.87 & - & - \\
\hline K-upgrad & K-effluent & K-effluent & K-crossgrad & - & K-effluent & K-effluent & - & - & & \\
\hline-1.40 & -1.72 & 0.21 & -6.85 & -3.61 & -4.4 & - & -6.85 & -6.77 & -3.54 & P82085 \\
\hline-8.50 & -7.20 & 2.20 & -43.50 & -15.10 & -21.6 & - & -44.00 & -43.2 & -14.20 & P82082 \\
\hline 7.53 & 15.35 & 17.58 & 6.96 & 14.68 & 23.07 & 24.71 & 5.27 & 5.09 & 2.36 & P82690 \\
\hline 3.19 & 6.77 & 10.13 & 1.97 & 8.18 & 11.30 & 12.23 & 2.13 & 1.09 & 2.55 & P63041 \\
\hline- & - & - & - & 27.34 & 17.25 & - & - & - & - & P82691 \\
\hline 40,300 & 28,100 & 50,800 & 3,250 & 960 & 3,160 & 3,680 & 2,300 & 1,610 & 276 & P00095 \\
\hline 25.7 & 17.3 & 33.3 & 1.70 & 0.47 & 1.65 & 1.93 & 1.18 & 0.81 & 0.13 & - \\
\hline 73.4 & 49.4 & 95.1 & 4.9 & 1.3 & 4.7 & 5.5 & 3.4 & 2.3 & 0.4 & - \\
\hline 26.6 & 50.6 & 4.9 & 95.1 & 98.7 & 95.3 & 94.5 & 96.6 & 97.7 & 99.6 & - \\
\hline 0.556 & 3.077 & 0.754 & 0.415 & - & - & - & - & - & - & - \\
\hline 0.234 & 0.274 & 0.265 & 0.103 & - & - & - & - & - & - & - \\
\hline- & - & - & - & 6.9 & 7.4 & - & 7.8 & 8.2 & 7.3 & P00400 \\
\hline- & - & - & - & 27.5 & 27.0 & - & 21.0 & 20.5 & 25.0 & P00010 \\
\hline- & - & - & - & 756 & 761 & - & 756 & 757 & 758 & P00025 \\
\hline- & - & - & - & 1.6 & 0.6 & - & 8.2 & 7.8 & 8.2 & P00300 \\
\hline- & - & - & - & 0.75 & 0.06 & - & 5.52 & 6.09 & & - \\
\hline
\end{tabular}


Table 4. Nutrients, stable isotopes, and physical parameters for water samples collected at Lahaina, Hawaii, May 21, 2008.

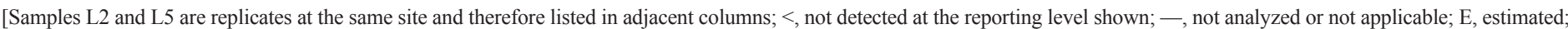

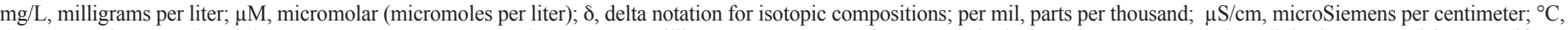

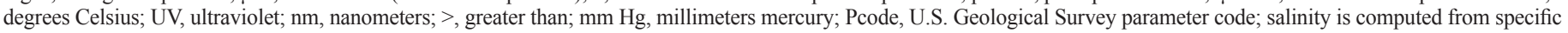

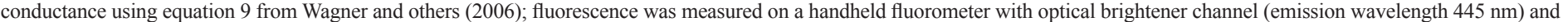

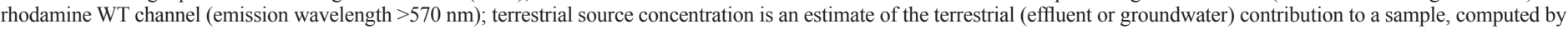
unmixing the seawater dilution it has undergone using Equation 1, this report]

\begin{tabular}{|c|c|c|c|c|c|c|}
\hline \multirow{6}{*}{ Constituent and category } & \multirow{3}{*}{$\begin{array}{r}\text { Sample } \\
\text { designation: } \\
\text { Type of sample: }\end{array}$} & \multicolumn{5}{|c|}{ Lahaina nearshore marine } \\
\hline & & L1 & $\mathbf{L} 2$ & $\mathbf{L 5}$ & L3 & L4 \\
\hline & & $\begin{array}{c}\text { Submarine } \\
\text { spring/inverted } \\
\text { funnel }\end{array}$ & $\begin{array}{c}\text { Submarine } \\
\text { spring/inverted } \\
\text { funnel }\end{array}$ & $\begin{array}{c}\text { Submarine } \\
\text { spring/inverted } \\
\text { funnel }\end{array}$ & Water column & Water column \\
\hline & Date: & 20080521 & 20080521 & 20080521 & 20080521 & 20080521 \\
\hline & Time: & 1150 & 1230 & 1020 & 1355 & 0915 \\
\hline & Station ID: & 205612156413101 & 205612156413102 & 205612156413105 & 205612156413103 & 205612156413104 \\
\hline Nutrients, dissolved, mass concentration (as reported by lab) & Units & & & & & \\
\hline Ammonium $\left(\mathrm{NH}_{4}\right)$, as $\mathrm{N}$ & $\mathrm{mg} / \mathrm{L}$ & E0.083 & 0.229 & $<0.200$ & $<0.200$ & $<0.200$ \\
\hline Nitrate plus nitrite $\left(\mathrm{NO}_{3}+\mathrm{NO}_{2}\right)$, as $\mathrm{N}$ & $\mathrm{mg} / \mathrm{L}$ & 0.746 & 0.496 & 0.870 & 0.022 & 0.042 \\
\hline Nitrite $\left(\mathrm{NO}_{2}\right)$, as $\mathrm{N}$ & $\mathrm{mg} / \mathrm{L}$ & - & - & - & - & - \\
\hline Dissolved inorganic nitrogen ( $\mathrm{DIN}=\mathrm{NO}_{2}+\mathrm{NO}_{3}+\mathrm{NH}_{4}$ ), as $\mathrm{N}$ & $\mathrm{mg} / \mathrm{L}$ & 0.829 & 0.725 & 0.870 & 0.022 & 0.042 \\
\hline Total dissolved nitrogen $(\mathrm{TDN}=\mathrm{DIN}+$ organic-N), as $\mathrm{N}$ & $\mathrm{mg} / \mathrm{L}$ & - & - & - & - & - \\
\hline Orthophosphate $\left(\mathrm{PO}_{4}\right)$, as $\mathrm{P}$ & $\mathrm{mg} / \mathrm{L}$ & 0.124 & 0.076 & 0.130 & 0.006 & E0.005 \\
\hline Total dissolved phosphorus (TDP), as P & $\mathrm{mg} / \mathrm{L}$ & - & - & - & - & - \\
\hline \multicolumn{7}{|l|}{ Nutrients, dissolved, molar concentration (computed) } \\
\hline Ammonium $\left(\mathrm{NH}_{4}\right)$, as $\mathrm{N}$ & $\mu \mathrm{M}$ & E5.9 & 16.3 & $<14$ & $<14$ & $<14$ \\
\hline Nitrate plus nitrite $\left(\mathrm{NO}_{3}+\mathrm{NO}_{2}\right)$, as $\mathrm{N}$ & $\mu \mathrm{M}$ & 53.3 & 35.4 & 62.1 & 1.6 & 3.0 \\
\hline Nitrite $\left(\mathrm{NO}_{2}\right)$, as $\mathrm{N}$ & $\mu \mathrm{M}$ & - & - & - & - & - \\
\hline Dissolved inorganic nitrogen (DIN), as N & $\mu \mathrm{M}$ & 59.2 & 51.7 & 62.1 & 1.6 & 3.0 \\
\hline Total dissolved nitrogen (TDN), as $\mathrm{N}$ & $\mu \mathrm{M}$ & - & - & - & - & - \\
\hline Organic nitrogen, by subtraction (organic-N=TDN-DIN), as $\mathrm{N}$ & $\mu \mathrm{M}$ & - & - & - & - & - \\
\hline Orthophosphate $\left(\mathrm{PO}_{4}\right)$, as $\mathrm{P}$ & $\mu \mathrm{M}$ & 4.0 & 2.5 & 4.2 & 0.2 & E0.16 \\
\hline Total dissolved phosphorus (TDP), as $\mathrm{P}$ & $\mu \mathrm{M}$ & - & - & - & - & - \\
\hline $\mathrm{N}: \mathrm{P}$ molar ratio $\left(\mathrm{DIN} / \mathrm{PO}_{4}\right)$ & - & 15 & 21 & 15 & 8 & 19 \\
\hline \multicolumn{7}{|l|}{ Terrestrial source concentration, seawater dilution removed } \\
\hline Source ammonium $\left(\mathrm{NH}_{4}\right)$, as $\mathrm{N}$ & $\mu \mathrm{M}$ & E24 & 105 & - & - & - \\
\hline Source nitrate plus nitrite $\left(\mathrm{NO}_{3}+\mathrm{NO}_{2}\right)$, as $\mathrm{N}$ & $\mu \mathrm{M}$ & 219 & 229 & 235 & 82 & 54 \\
\hline Source dissolved inorganic nitrogen (DIN), as N & $\mu \mathrm{M}$ & 243 & 333 & 235 & 80 & 54 \\
\hline Source orthophosphate $\left(\mathrm{PO}_{4}\right)$, as $\mathrm{P}$ & $\mu \mathrm{M}$ & 16 & 15 & 16 & 4.8 & E0.9 \\
\hline Terrestrial parent water used for seawater dilution adjustment & - & L12-effluent & L12-effluent & L12-effluent & L12-effluent & L11-well \\
\hline \multicolumn{7}{|l|}{ Stable isotopes } \\
\hline$\delta^{2} \mathrm{H}$ (Deuterium/Protium ratio) in water & per mil & -0.50 & 1.40 & -1.30 & 3.30 & 2.00 \\
\hline$\delta^{18} \mathrm{O}$ (Oxygen-18/Oxygen-16 ratio) in water & per mil & -0.53 & -0.06 & -0.56 & 0.34 & 0.27 \\
\hline$\delta^{15}{\mathrm{~N}-\mathrm{NO}_{3}}_{3}$ (Nitrogen-15/Nitrogen-14 ratio) in nitrate fraction & per mil & 39.31 & 39.68 & 39.75 & 17.00 & 6.18 \\
\hline$\delta^{18}{\mathrm{O}-\mathrm{NO}_{3}}_{\text {(Oxygen-18/Oxygen-16 ratio) in nitrate fraction }}$ & per mil & 18.72 & 18.84 & 18.84 & 16.70 & 6.25 \\
\hline \multicolumn{7}{|l|}{ Physical parameters, field measurements } \\
\hline Specific conductance & $\mu \mathrm{S} / \mathrm{cm}$ at $25^{\circ} \mathrm{C}$ & 41,700 & 45,900 & 40,700 & 52,300 & 50,600 \\
\hline Salinity (dimensionless), computed from specific conductance & - & 26.7 & 29.7 & 26.0 & 34.4 & 33.2 \\
\hline Seawater fraction, computed from salinity & percent & 76.3 & 84.9 & 74.3 & 98.3 & 94.9 \\
\hline Freshwater fraction, computed from salinity & percent & 23.7 & 15.1 & 25.7 & 1.7 & 5.1 \\
\hline Fabric brightener fluorescence at $445 \mathrm{~nm}$, meter reading & meter units & 3.129 & 1.944 & 2.893 & 0.140 & 0.927 \\
\hline Rhodamine WT fluorescence at $>570 \mathrm{~nm}$, meter reading & meter units & 0.220 & 0.224 & 0.210 & 0.284 & 0.253 \\
\hline
\end{tabular}


waters, groundwater, and wastewater effluent (2008)

L6

\section{Water}

column

20080521

1405 205612156413106

\section{L7}

\section{L8}

Water

column

Water

column

20080521

1415

20080521

1440 $205612156413107 \quad 205612156413108$

\section{เ9}

Water

Column
20080521
1455

\section{L10}

Lagoon

water

column

20080521

1505

205612156413109

205612156413110

$\begin{array}{cc}\text { L11-well } & \text { L12-effluent } \\ \text { Upland well } & \begin{array}{c}\text { Lahaina WWRF } \\ \text { treated effluent }\end{array} \\ 20080522 & 20080521 \\ 1045 & 0750\end{array}$

205559156402801
205646156411200

$\begin{array}{ccccccrr}<0.200 & <0.200 & <0.200 & <0.200 & <0.200 & \text { E0.010 } & 2.65 & \text { P00608 } \\ 0.030 & 0.041 & 0.061 & 0.017 & 3.31 & 3.55 & \text { P00631 } \\ - & - & - & - & - & - & 0.250 & \text { P00613 } \\ 0.030 & 0.041 & 0.061 & 0.017 & 3.31 & 3.56 & 5.12\end{array}$

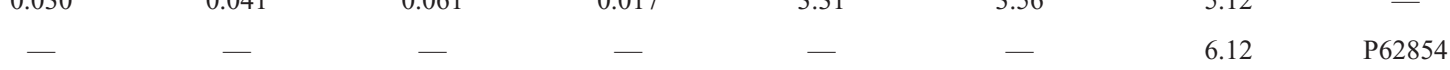

$\begin{array}{lllllllll}0.007 & 0.008 & 0.009 & \text { E0.005 } & 0.061 & 0.240 & 0.154 & P 00671\end{array}$

$\begin{array}{lllllll}- & - & - & - & - & 0.189 & P 0666\end{array}$

$\begin{array}{llllllll}<14 & <14 & <14 & <14 & \text { E0.7 } & 189 & \text { P00608 }\end{array}$

2.1

2.9

$$
4.4
$$

1.2

$$
236
$$

-

\section{1}

$-$

-

- -

0.2

$-$

9

-

\begin{abstract}
4.4
\end{abstract}
$-$

$-$

0.3

-

15

1.2

-

$-$

E0.16

-

8

- -

$83 \quad 106$

$85 \quad 107$

5.0

$$
5.8
$$

135

133

5.7

L12-effluent

L12-effluent

L12-effluent

\begin{tabular}{|c|c|c|c|c|c|c|c|}
\hline 4.40 & 5.10 & 2.90 & 1.80 & -12.30 & -14.00 & -10.90 & P82082 \\
\hline 0.42 & 0.28 & 0.30 & 0.38 & -3.09 & -3.76 & -3.21 & P82085 \\
\hline 24.74 & 30.63 & 30.77 & 12.50 & 12.80 & 1.17 & 22.66 & P82690 \\
\hline 16.66 & 17.73 & 15.85 & 14.11 & 6.64 & 2.72 & 8.20 & P63041 \\
\hline 52,000 & 51,900 & 51,600 & 52,300 & 4,810 & 947 & 2,030 & P00095 \\
\hline 34.2 & 34.1 & 33.9 & 34.4 & 2.57 & 0.47 & 1.03 & - \\
\hline 97.7 & 97.4 & 96.9 & 98.3 & 7.3 & 1.3 & 2.9 & - \\
\hline 2.3 & 2.6 & 3.1 & 1.7 & 92.7 & 98.7 & 97.1 & - \\
\hline 0.084 & 0.062 & -0.001 & -0.112 & 4.058 & 0.836 & 31.70 & - \\
\hline 0.229 & 0.165 & 0.233 & 0.113 & 0.592 & 0.243 & 0.457 & - \\
\hline
\end{tabular}

L11-well

L11-well 
Table 5. Pharmaceuticals and waste indicator compounds in water samples collected at Kihei, Hawaii, May 20, 2008, and November 15-17, 2004.

Concentrations in micrograms per liter, surrogate recovery in percent; detected compounds are bold and shaded; <, not detected at the laboratory reporting level (LRL) shown; E, estimated; M, presence of material verified but not quantified;--, not applicable; analyzed by USGS National Water Quality Laboratory using pharmaceuticals Schedule 2080 (custom lab code 9003 in 2004) for analysis of filtered samples (Furlong and others, 2008) and wastewater compounds Schedule 4433 for analysis of whole water (Zaugg and others, 2006); the filtered equivalent (Schedule 1433) was used in 2004; 2004 data are from Hunt (2007)]

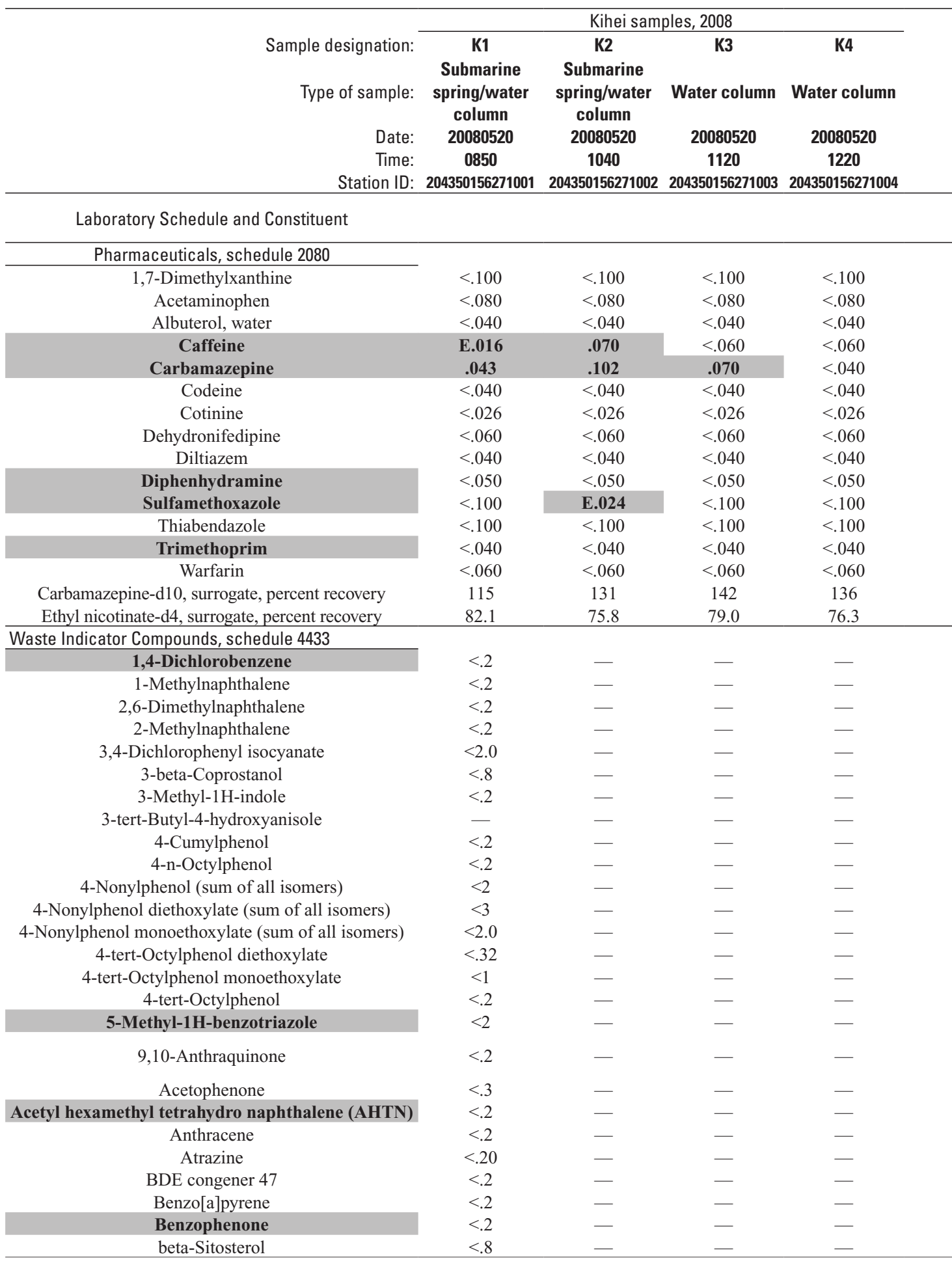

${ }^{1}$ Not a conclusive detection; present in blank, and sample concentration was not more than 10 times the blank concentration of $0.0070 \mathrm{mg} / \mathrm{L}$.

${ }^{2}$ Not a conclusive detection; present in blank, and sample concentration was not more than 10 times the blank concentration of $0.0106 \mathrm{mg} / \mathrm{L}$.

${ }^{3}$ Not a conclusive detection; present in blank, and sample concentration was not more than 10 times the blank concentration of $0.0086 \mathrm{mg} / \mathrm{L}$

${ }^{4}$ Could not be detected under experimental conditions; LRL not determined. 


\begin{tabular}{cccc}
\multicolumn{4}{c}{ Kihei samples, 2004 } \\
\hline K-effluent & K-downgrad & K-crossgrad & K-upgrad \\
Kihei WWRF & Down-gradient & Cross-gradient & Upgradient \\
treated effluent & well & well & well \\
20041117 & 20041115 & 20041115 & 20041116 \\
1100 & 1100 & 1500 & 1100 \\
204411156263800 & 204423156272501 & 204334156264301 & 204456156264101
\end{tabular}

\begin{tabular}{|c|c|c|c|c|c|}
\hline \multicolumn{6}{|c|}{ Pharmaceuticals, lab code 9003} \\
\hline$<.144$ & $<.144$ & $<.144$ & $<.144$ & Caffeine metabolite & P62030 \\
\hline$<.036$ & $<.036$ & $<.036$ & $<.036$ & Antipyretic & P62000 \\
\hline - & - & - & - & Antiasthmatic & P 62020 \\
\hline$<.016$ & $<.016$ & $<.016$ & $<.016$ & Stimulant & P50305 \\
\hline 0.111 & 0.114 & $<.011$ & $<.011$ & Anticonvulsant & P62793 \\
\hline$<.015$ & $<.015$ & $<.015$ & $<.015$ & Analgesic & P62003 \\
\hline 0.035 & $<.014$ & $<.014$ & $<.014$ & Nicotine metabolite & P62005 \\
\hline $0.022^{1}$ & $0.0147^{2}$ & $<.015$ & $<.015$ & Antianginal & P62004 \\
\hline - & - & - & - & Antihypertensive & P62008 \\
\hline 0.129 & $<.015$ & $<.015$ & $0.0108^{3}$ & Antihistamine & P62796 \\
\hline$<.064$ & $<.064$ & $<.064$ & $<.064$ & Antibiotic & P62021 \\
\hline$<.011$ & $<.011$ & $<.011$ & $<.011$ & Fungicide & P62801 \\
\hline 0.025 & $<.013$ & $<.013$ & $<.013$ & Antibiotic & P62023 \\
\hline$<.012$ & $<.012$ & $<.012$ & $<.012$ & Anticoagulant & P62024 \\
\hline - & - & - & - & Surrogate & P90797 \\
\hline - & - & - & - & Surrogate & P99571 \\
\hline \multicolumn{6}{|c|}{ Waste Indicator Compounds, schedule 1433} \\
\hline E.2 & E.1 & $<.5$ & $<.5$ & Deodorizer & P34571 \\
\hline$<.5$ & $<.5$ & $<.5$ & $<.5$ & PAH, pesticide adjuvant, wall coverings & P81696 \\
\hline$<.5$ & $<.5$ & $<.5$ & $<.5$ & PAH, pesticide adjuvant & P62805 \\
\hline$<.5$ & $<.5$ & $<.5$ & $<.5$ & PAH, pesticide adjuvant, sealant & P30194 \\
\hline- & - & - & - & Plastic additive; intermediate in dyes & P63145 \\
\hline$<2$ & $<2$ & $<2$ & $<2$ & Fecal sterol, naturally occurring & P62806 \\
\hline$<1$ & $<1$ & $<1$ & $<1$ & Fragrance & P62807 \\
\hline$<5$ & $<5$ & $<5$ & $<5$ & Antioxidant, food preservative & P62059 \\
\hline$<1$ & $<1$ & $<1$ & $<1$ & Nonionic detergent metabolite, surfactant & P62808 \\
\hline$<1$ & $<1$ & $<1$ & $<1$ & Nonionic detergent metabolite, surfactant & P62809 \\
\hline$<5$ & $<5$ & $<5$ & $<5$ & Nonionic detergent metabolite, surfactant & P 62829 \\
\hline$<5$ & $<5$ & $<5$ & $<5$ & Nonionic detergent metabolite, surfactant & P61703 \\
\hline - & - & - & - & Nonionic detergent metabolite, surfactant & P61704 \\
\hline$<1$ & $<1$ & $<1$ & $<1$ & Nonionic detergent metabolite, surfactant & P61705 \\
\hline$<1$ & $<1$ & $<1$ & $<1$ & Nonionic detergent metabolite, surfactant & P61706 \\
\hline$<1$ & $<1$ & $<1$ & $<1$ & Nonionic detergent metabolite, surfactant & P62810 \\
\hline M & $<2$ & $<2$ & $<2$ & Manufacturing additive; anticorrosive & P61944 \\
\hline$<.5$ & $<.5$ & $<.5$ & $<.5$ & $\begin{array}{c}\text { Manufacturing of dyes/textiles, seed treatment, bird } \\
\text { repellant }\end{array}$ & P 62813 \\
\hline$<.5$ & $<.5$ & $<.5$ & $<.5$ & Fragrance; solvent & P62811 \\
\hline .7 & $<.5$ & $<.5$ & $<.5$ & Fragrance: musk & P 62812 \\
\hline$<.5$ & $<.5$ & $<.5$ & $<.5$ & PAH, combustion product, used in dyes & P34220 \\
\hline - & - & - & - & Herbicide & P39630 \\
\hline- & - & - & - & Fire retardant & P63147 \\
\hline$<.5$ & $<.5$ & $<.5$ & $<.5$ & PAH, combustion product & P34247 \\
\hline E.1 & $<.5$ & $<.5$ & $<.5$ & Fragrance, hair mousse, inks & P62814 \\
\hline$<2$ & $<2$ & $<2$ & $<2$ & Plant sterol, naturally occurring & P62815 \\
\hline
\end{tabular}


Table 5. Pharmaceuticals and waste indicator compounds in water samples collected at Kihei, Hawaii, May 20, 2008, and November 15-17, 2004-Continued.

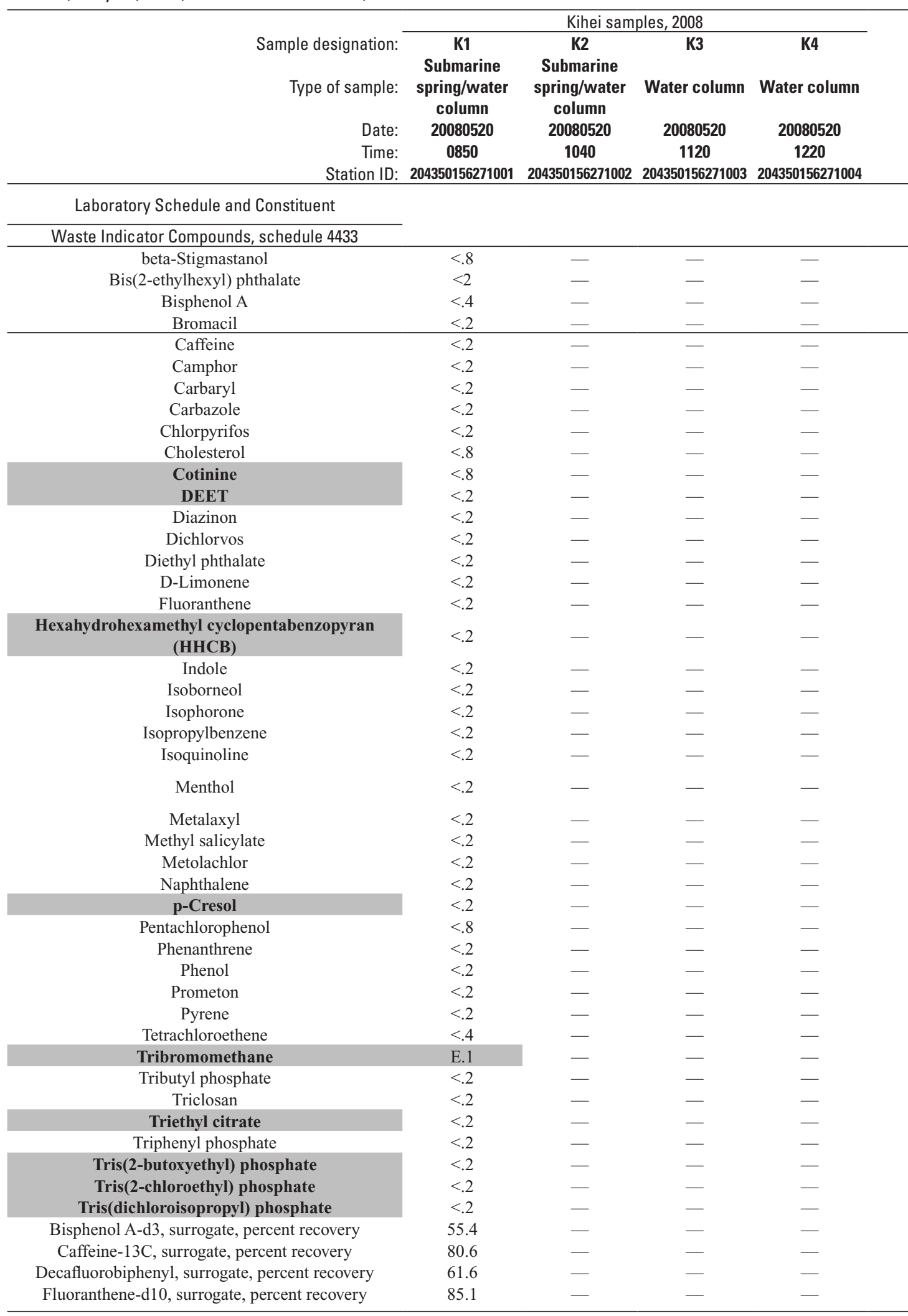

\footnotetext{
${ }^{1}$ Not a conclusive detection; present in blank, and sample concentration was not more than 10 times the blank concentration of $0.0070 \mathrm{mg} / \mathrm{L}$.

${ }^{2}$ Not a conclusive detection; present in blank, and sample concentration was not more than 10 times the blank concentration of $0.0106 \mathrm{mg} / \mathrm{L}$.

${ }^{3}$ Not a conclusive detection; present in blank, and sample concentration was not more than 10 times the blank concentration of $0.0086 \mathrm{mg} / \mathrm{L}$.

${ }^{4}$ Could not be detected under experimental conditions; LRL not determined.
} 
Kihei samples, 2004

\begin{tabular}{cccc}
\hline K-effluent & K-downgrad & K-crossgrad & K-upgrad \\
$\begin{array}{c}\text { Kihei WWRF } \\
\text { treated effluent }\end{array}$ & $\begin{array}{c}\text { Down- gradient } \\
\text { well }\end{array}$ & $\begin{array}{c}\text { Cross-gradient } \\
\text { well }\end{array}$ & $\begin{array}{c}\text { Upgradient } \\
\text { well }\end{array}$ \\
20041117 & 20041115 & 20041115 & 20041116 \\
1100 & 1100 & 1500 & 1100 \\
204411156263800 & 204423156272501 & 204334156264301 & 204456156264101 \\
\hline
\end{tabular}

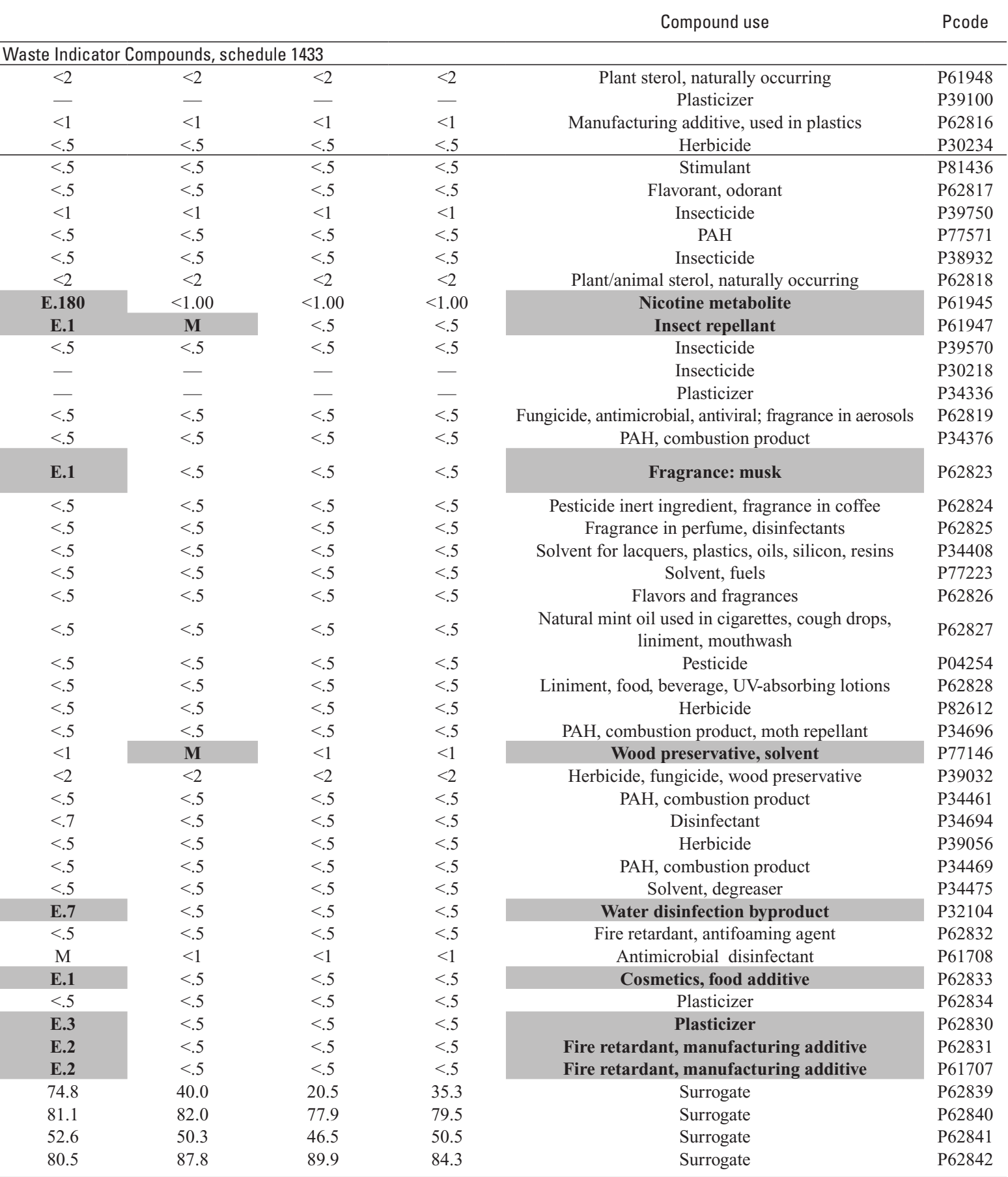


Table 6. Pharmaceuticals and waste indicator compounds in water samples collected at Lahaina, Hawaii, May 21, 2008.

[Concentrations in micrograms per liter, surrogate recovery in percent; detected compounds are bold and shaded; <, not detected at the laboratory reporting level (LRL) shown; E, estimated; M, presence of material verified but not quantified; V, known to be an artifact of a dechlorination agent added to sample; —, not applicable; analyzed by USGS National Water Quality Laboratory using pharmaceuticals Schedule 2080 (custom lab code 9003 in 2004) for analysis of filtered samples (Furlong and others, 2008) and wastewater compounds Schedule 4433 for analysis of whole water (Zaugg and others, 2006)]

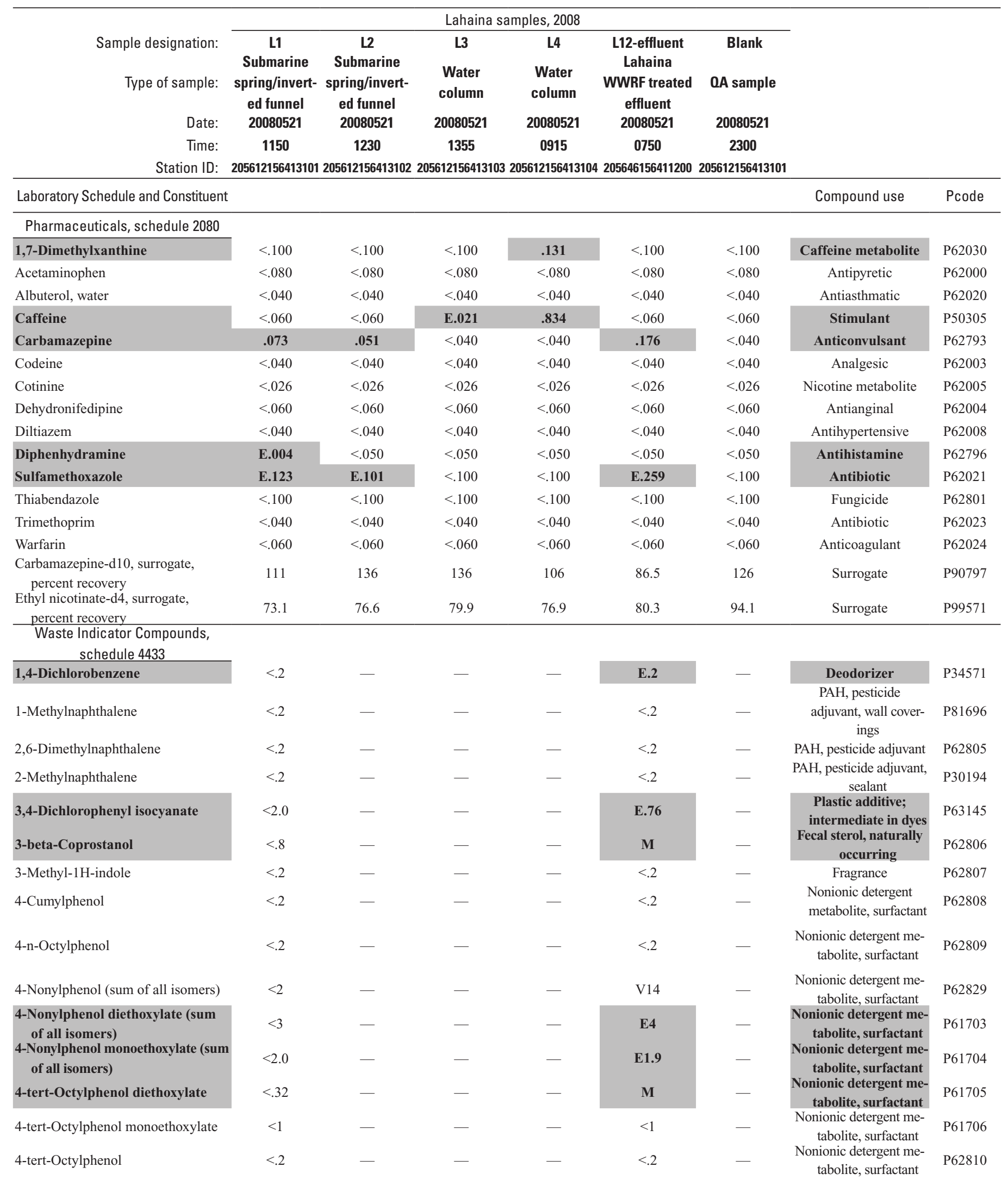


Table 6. Pharmaceuticals and waste indicator compounds in water samples collected at Lahaina, Hawaii, May 21, 2008_-Continued.

\begin{tabular}{|c|c|c|c|c|c|c|c|c|}
\hline & \multicolumn{6}{|c|}{ Lahaina samples, 2008} & & \\
\hline Type of sample: & $\begin{array}{c}\text { L1 } \\
\text { Submarine } \\
\text { spring/invert- } \\
\text { ed funnel }\end{array}$ & $\begin{array}{c}\text { L2 } \\
\text { Submarine } \\
\text { spring/invert- } \\
\text { ed funnel }\end{array}$ & $\begin{array}{c}\text { L3 } \\
\text { Water } \\
\text { column }\end{array}$ & $\begin{array}{c}\text { L4 } \\
\text { Water } \\
\text { column }\end{array}$ & $\begin{array}{l}\text { L12-effluent } \\
\text { Lahaina } \\
\text { WWRF treated } \\
\text { effluent }\end{array}$ & $\begin{array}{c}\text { Blank } \\
\text { OA sample }\end{array}$ & & \\
\hline Date: & 20080521 & 20080521 & 20080521 & 20080521 & 20080521 & 20080521 & & \\
\hline Time: & 1150 & 1230 & 1355 & 0915 & 0750 & 2300 & & \\
\hline \multicolumn{9}{|c|}{ Station ID: 205612156413101205612156413102205612156413103205612156413104205646156411200205612156413101} \\
\hline Laboratory Schedule and Constituent & & & & & & & Compound use & Pcode \\
\hline \multicolumn{9}{|l|}{$\begin{array}{l}\text { Waste Indicator Compounds, } \\
\text { schedule } 4433\end{array}$} \\
\hline 5-Methyl-1H-benzotriazole & $<2$ & - & - & - & $\mathbf{M}$ & - & $\begin{array}{l}\text { Manufacturing addi- } \\
\text { tive; anticorrosive }\end{array}$ & P61944 \\
\hline 9,10 -Anthraquinone & $<.2$ & - & - & - & $<.2$ & - & $\begin{array}{l}\text { Manufacturing of dyes/ } \\
\text { textiles, seed treat- } \\
\text { ment, bird repellant }\end{array}$ & P 62813 \\
\hline Acetophenone & $<.3$ & - & - & - & $<.3$ & - & Fragrance; solvent & P62811 \\
\hline $\begin{array}{l}\text { Acetyl hexamethyl tetrahydro } \\
\text { naphthalene (AHTN) }\end{array}$ & M & - & - & - & E.2 & - & Fragrance: musk & P62812 \\
\hline Anthracene & $<.2$ & - & - & - & $<.2$ & - & $\begin{array}{l}\text { PAH, combustion prod- } \\
\text { uct, used in dyes }\end{array}$ & P 34220 \\
\hline Atrazine & $<.20$ & - & - & - & $<.20$ & - & Herbicide & P39630 \\
\hline BDE congener 47 & $<.2$ & - & - & - & $<.2$ & - & Fire retardant & P63147 \\
\hline Benzo[a]pyrene & $<.2$ & - & - & - & $<.2$ & - & $\begin{array}{c}\mathrm{PAH}, \text { combustion } \\
\text { product }\end{array}$ & P 34247 \\
\hline Benzophenone & $<.2$ & - & - & - & .3 & - & $\begin{array}{l}\text { Fragrance, hair } \\
\text { mousse, inks }\end{array}$ & P 62814 \\
\hline beta-Sitosterol & $<.8$ & - & - & - & $\mathbf{M}$ & - & & P62815 \\
\hline beta-Stigmastanol & $<.8$ & - & - & - & $\mathbf{M}$ & - & $\begin{array}{c}\text { Plant sterol, naturally } \\
\text { occurring }\end{array}$ & P61948 \\
\hline Bis(2-ethylhexyl) phthalate & $<2$ & - & - & - & $<2$ & - & Plasticizer & P39100 \\
\hline Bisphenol A & $<.4$ & - & - & - & $<.4$ & - & $\begin{array}{c}\text { Manufacturing additive, } \\
\text { used in plastics }\end{array}$ & P62816 \\
\hline Bromacil & $<.2$ & - & - & - & $<.2$ & - & Herbicide & P30234 \\
\hline Caffeine & $<.2$ & - & - & - & $<.2$ & - & Stimulant & P81436 \\
\hline Camphor & $<.2$ & - & - & - & $<.2$ & - & Flavorant, odorant & P62817 \\
\hline Carbaryl & $<.2$ & - & - & - & $<.2$ & - & Insecticide & P39750 \\
\hline Carbazole & $<.2$ & - & - & - & $<.2$ & - & РAH & P77571 \\
\hline Chlorpyrifos & $<.2$ & - & - & - & $<.2$ & - & Insecticide & P38932 \\
\hline Cholesterol & $<.8$ & - & - & - & M & - & $\begin{array}{l}\text { Plant/animal sterol, } \\
\text { naturally occurring }\end{array}$ & P62818 \\
\hline Cotinine & $<.8$ & - & - & - & $<.8$ & - & Nicotine metabolite & P61945 \\
\hline DEET & $<.2$ & - & - & - & $<.2$ & - & Insect repellant & P61947 \\
\hline Diazinon & $<.2$ & - & - & - & $<.2$ & - & Insecticide & P39570 \\
\hline Dichlorvos & $<.2$ & - & - & - & $<.2$ & - & Insecticide & P30218 \\
\hline Diethyl phthalate & $<.2$ & - & - & - & $<.2$ & - & Plasticizer & P34336 \\
\hline D-Limonene & $<.2$ & - & - & - & $<.2$ & - & $\begin{array}{l}\text { Fungicide, antimi- } \\
\text { crobial, antiviral; } \\
\text { fragrance in aerosols }\end{array}$ & P62819 \\
\hline Fluoranthene & $<.2$ & - & - & - & $<.2$ & - & $\begin{array}{l}\text { PAH, combustion } \\
\text { product }\end{array}$ & P34376 \\
\hline $\begin{array}{l}\text { Hexahydrohexamethyl } \\
\text { cyclopentabenzopyran (HHCB) }\end{array}$ & E.1 & - & - & - & 1.5 & - & Fragrance: musk & P62823 \\
\hline Indole & $<.2$ & - & - & - & $<.2$ & - & $\begin{array}{c}\text { Pesticide inert } \\
\text { ingredient, fragrance } \\
\text { in coffee }\end{array}$ & P62824 \\
\hline Isoborneol & $<.2$ & - & - & - & $<.2$ & - & $\begin{array}{l}\text { Fragrance in perfume, } \\
\text { disinfectants }\end{array}$ & P62825 \\
\hline Isophorone & $<.2$ & - & - & - & $\mathbf{M}$ & - & $\begin{array}{l}\text { Solvent for lacquers, } \\
\text { plastics, oils, silicon, } \\
\text { resins }\end{array}$ & P34408 \\
\hline Isopropylbenzene & $<.2$ & - & - & - & $<.2$ & - & Solvent, fuels & P77223 \\
\hline
\end{tabular}


Table 6. Pharmaceuticals and waste indicator compounds in water samples collected at Lahaina, Hawaii, May 21, 2008-Continued.

\begin{tabular}{|c|c|c|c|c|c|c|c|c|}
\hline & \multicolumn{6}{|c|}{ Lahaina samples, 2008} & & \\
\hline $\begin{array}{r}\text { Type of sample: } \\
\text { Date: } \\
\text { Time: }\end{array}$ & $\begin{array}{c}\text { L1 } \\
\text { Submarine } \\
\text { spring/invert- } \\
\text { ed funnel } \\
20080521 \\
1150\end{array}$ & $\begin{array}{c}\text { L2 } \\
\text { Submarine } \\
\text { spring/invert- } \\
\text { ed funnel } \\
20080521 \\
1230\end{array}$ & $\begin{array}{c}\text { L3 } \\
\text { Water } \\
\text { column } \\
20080521 \\
1355\end{array}$ & $\begin{array}{c}\text { L4 } \\
\text { Water } \\
\text { column } \\
20080521 \\
0915\end{array}$ & $\begin{array}{c}\text { L12-effluent } \\
\text { Lahaina } \\
\text { WWRF treated } \\
\text { effluent } \\
20080521 \\
0750\end{array}$ & $\begin{array}{c}\text { Blank } \\
\text { OA sample } \\
20080521 \\
2300\end{array}$ & & \\
\hline \multicolumn{9}{|c|}{ Station ID: 205612156413101205612156413102205612156413103205612156413104205646156411200205612156413101} \\
\hline Laboratory Schedule and Constituent & & & & & & & Compound use & Pcode \\
\hline \multicolumn{9}{|l|}{$\begin{array}{l}\text { Waste Indicator Compounds, } \\
\text { schedule } 4433\end{array}$} \\
\hline Isoquinoline & $<.2$ & - & - & - & $<.2$ & - & $\begin{array}{l}\text { Flavors and fragrances } \\
\text { Natural mint oil used }\end{array}$ & $\mathrm{P} 62826$ \\
\hline Menthol & $<.2$ & - & - & - & $<.2$ & - & $\begin{array}{l}\text { in cigarettes, cough } \\
\text { drops, liniment, } \\
\text { mouthwash }\end{array}$ & $\mathrm{P} 62827$ \\
\hline Metalaxyl & $<.2$ & - & - & - & $<.2$ & - & Pesticide & P04254 \\
\hline Methyl salicylate & $<.2$ & - & - & - & $<.2$ & - & $\begin{array}{c}\text { Liniment, food, bever- } \\
\text { age, UV-absorbing } \\
\text { lotions }\end{array}$ & $\mathrm{P} 62828$ \\
\hline Metolachlor & $<.2$ & - & - & - & $<.2$ & - & Herbicide & P82612 \\
\hline Naphthalene & $<.2$ & - & - & - & $<.2$ & - & $\begin{array}{c}\mathrm{PAH} \text {, combustion } \\
\text { product, moth } \\
\text { repellant }\end{array}$ & P34696 \\
\hline p-Cresol & $<.2$ & - & - & - & M & - & $\begin{array}{c}\text { Wood preservative, } \\
\text { solvent }\end{array}$ & P77146 \\
\hline Pentachlorophenol & $<.8$ & - & - & - & M & - & $\begin{array}{l}\text { Herbicide, fungicide, } \\
\text { wood preservative }\end{array}$ & P39032 \\
\hline Phenanthrene & $<.2$ & - & - & - & $<.2$ & - & $\begin{array}{c}\mathrm{PAH}, \text { combustion } \\
\text { product }\end{array}$ & P34461 \\
\hline Phenol & $<.2$ & - & - & - & E.1 & - & Disinfectant & P34694 \\
\hline Prometon & $<.2$ & - & - & - & $<.2$ & - & Herbicide & P39056 \\
\hline Pyrene & $<.2$ & - & - & - & $<.2$ & - & $\begin{array}{l}\mathrm{PAH}, \text { combustion } \\
\text { product }\end{array}$ & P34469 \\
\hline Tetrachloroethene & $<.4$ & - & - & - & $<.4$ & - & Solvent, degreaser & P34475 \\
\hline Tribromomethane & E.1 & - & - & - & E.1 & - & $\begin{array}{l}\text { Water disinfection } \\
\text { byproduct }\end{array}$ & P32104 \\
\hline Tributyl phosphate & $<.2$ & - & - & - & E.1 & - & $\begin{array}{l}\text { Fire retardant, anti- } \\
\text { foaming agent }\end{array}$ & P62832 \\
\hline Triclosan & $<.2$ & - & - & - & M & - & $\begin{array}{l}\text { Antimicrobial disin- } \\
\text { fectant }\end{array}$ & P61708 \\
\hline Triethyl citrate & $<.2$ & - & - & - & E.1 & - & $\begin{array}{l}\text { Cosmetics, food ad- } \\
\text { ditive }\end{array}$ & P62833 \\
\hline Triphenyl phosphate & $<.2$ & - & - & - & $\mathbf{M}$ & - & Plasticizer & $\mathrm{P} 62834$ \\
\hline Tris(2-butoxyethyl) phosphate & E.1 & - & - & - & E1.7 & - & Plasticizer & $\mathrm{P} 62830$ \\
\hline Tris(2-chloroethyl) phosphate & $<.2$ & - & - & - & .2 & - & $\begin{array}{l}\text { Fire retardant, manu- } \\
\text { facturing additive }\end{array}$ & P62831 \\
\hline Tris(dichloroisopropyl) phosphate & E.1 & - & - & - & .3 & - & $\begin{array}{l}\text { Fire retardant, manu- } \\
\text { facturing additive }\end{array}$ & P61707 \\
\hline $\begin{array}{l}\text { Bisphenol A-d3, surrogate, } \\
\text { percent recovery }\end{array}$ & 61.2 & - & - & - & 56.6 & - & Surrogate & P62839 \\
\hline $\begin{array}{l}\text { Caffeine-13C, surrogate, } \\
\text { percent recovery }\end{array}$ & 86.2 & - & - & - & 81.7 & - & Surrogate & $\mathrm{P} 62840$ \\
\hline $\begin{array}{l}\text { Decafluorobiphenyl, surrogate, } \\
\text { percent recovery }\end{array}$ & 57.9 & - & - & - & 61.5 & - & Surrogate & P62841 \\
\hline $\begin{array}{l}\text { Fluoranthene-d10, surrogate, } \\
\text { percent recovery }\end{array}$ & 85.1 & - & - & - & 78.6 & - & Surrogate & $\mathrm{P} 62842$ \\
\hline
\end{tabular}


effluent. Effluent and wells were referenced to themselves and resulted in no adjustment, except for the downgradient well, which was adjusted to effluent salinity because it is known to contain a large fraction of effluent. At Lahaina, samples L4, L9, and L10 were adjusted to salinity in upland well L11 because overall chemistry indicates they are more closely related to upland groundwater than to effluent. Remaining samples were adjusted to effluent salinity, and both effluent and the upland well sample were referenced to themselves.

\section{Stable Isotopes and Isotope Notation}

Stable isotopes can indicate water and nutrient origins under favorable circumstances. Isotopic compositions measured in this study included $\delta^{15} \mathrm{~N}$ and $\delta^{18} \mathrm{O}$ of dissolved nitrate $\left(\mathrm{NO}_{3}\right), \delta^{15} \mathrm{~N}$ of algal-tissue nitrogen, and $\delta^{2} \mathrm{H}$ and $\delta^{18} \mathrm{O}$ of water (the water molecule itself, $\mathrm{H}_{2} \mathrm{O}$ ). Two samples from 2004 had sufficient ammonium for measurement of $\delta^{15} \mathrm{~N}$ of dissolved ammonium $\left(\mathrm{NH}_{4}\right)$. The various isotopic measures are ratios of a heavier isotope to a lighter isotope for a given element $\left({ }^{15} \mathrm{~N}\right.$ to ${ }^{14} \mathrm{~N},{ }^{18} \mathrm{O}$ to ${ }^{16} \mathrm{O},{ }^{2} \mathrm{H}$ to $\left.{ }^{1} \mathrm{H}\right)$. The $\delta$ symbol signifies "delta notation" and is read as "delta-N-15," "deltaO-18," and "delta-H-2." Units are parts per thousand ("per mil," denoted by the \%o symbol) and normalized to reference standards: atmospheric air (AIR) for N and Vienna Standard Mean Ocean Water (VSMOW) for O and H. Isotopic compositions can be positive or negative, with larger values referred to as "heavier" and smaller or more negative values as "lighter." Further explanation can be found in texts on isotope chemistry, for example Kendall and McDonnell (1998).

\section{Sample Collection, Processing, and Analysis}

Laboratory water samples were collected and preserved following USGS sampling and quality assurance (QA) protocols (U.S. Geological Survey, variously dated). Water and algae samples were analyzed at the USGS National Water Quality Laboratory (NWQL) and Reston Stable Isotope Laboratory (RSIL) using methods and quality-assurance protocols established at those laboratories. Table 1 lists types of analyses and USGS method codes (schedules and lab codes).

As with the screening samples, most laboratory water samples were collected by dipping bottles just below the ocean surface (less than $10 \mathrm{~cm}$ ), uncapping and capping bottles while submerged. Exceptions were Lahaina samples L1, L2, and L5, which were collected by inverted-funnel sampling to guide buoyant spring flow into sample bottles (collected by Meghan Dailer and Darla White using scuba). Sample bottles were capped underwater once filled and then passed to shore and placed in an iced cooler and later filtered and refrigerated before shipping on ice. Treated effluent at Lahaina WWRF was collected by pouring from their dip-pole sampler cup directly into sample bottles containing ascorbic acid as a dechlorinating reagent. At the upland Puukolii well, sample bottles were filled directly from a hose-bib tap. Field measurements (specific conductance and fabric brightener fluorescence) were made on all water samples using portable handheld meters.

Nutrient samples were collected in 1-liter polyethylene bottles rinsed with sample water and placed on ice. Samples were filtered at $0.45 \mu \mathrm{m}$ into $125-\mathrm{mL}$ amber polyethylene bottles prerinsed with filtered sample and shipped in iced coolers. Nitrogen-isotope samples (also filtered into 125-mL amber polyethylene bottles) were frozen until nitrate concentrations came back from the lab and then shipped.

Pharmaceutical and waste indicator samples were collected in 1-liter baked glass bottles with no prerinsing (as per method guidance), placed on ice, and shipped in iced coolers. Pharmaceuticals samples were filtered at the lab, whereas waste indicator samples were analyzed unfiltered.

Hydrogen- and oxygen-isotope samples were poured unfiltered from the 1-liter polyethylene bottles into $60-\mathrm{mL}$ clear glass bottles with no prerinsing, as per method guidance.

Specific conductance was measured on unfiltered samples with a YSI EC-300 conductivity meter after calibrating to a $50,000 \mu \mathrm{S} / \mathrm{cm}$ standard solution. Fluorescence was measured on unfiltered samples with a Turner Designs Aquafluor handheld fluorometer with one channel tuned for fabric optical brighteners (excitation UV-375 nm, emission $445 \mathrm{~nm}$ ) and the other for rhodamine WT fluorescent dye (excitation $540 \mathrm{~nm}$, emission $>570 \mathrm{~nm}$ ). Three brightener readings were taken and the middle value reported, refilling the cuvette each time; only one rhodamine reading was taken because these readings were much less variable. The fluorometer was not recalibrated to standard solutions but instead was operated at the manufacturer calibration.

\section{Quality Assurance}

Laboratory quality was assured by the analyzing laboratories. The USGS National Water-Quality Laboratory (NWQL) and Reston Stable-Isotope Laboratory (RSIL) maintain rigorous quality-assurance procedures, including blanks, spikerecovery analysis, and determination of laboratory reporting levels.

Field quality assurance consisted of one pharmaceuticals blank and calibration of instruments to known standards. For the blank, nitrogen-purged VOC-grade organic blank water was poured into a sample bottle in the field while gloved and with no rinsing. This emulated the corresponding field sampling (water-column samples were simply dipped while gloved, with no rinse and using no other equipment). Calibration books were maintained for instruments used.

\section{Benthic Macroalgae Samples}

Roughly 20 benthic macroalgae samples were collected per locale (19 at Kihei and 21 at Lahaina) by M. Dailer and D. White while snorkeling (table 7). Samples of the green macroalga Ulva sp. in growth position were hand-picked and placed in zip-lock bags, which were transferred to an 
Table 7. Nitrogen stable isotope composition and nitrogen dry-weight concentration in Ulva sp. macroalgae samples collected at Kihei and Lahaina, Hawaii, May 7- 28, 2008.

$[\delta$, delta notation for isotopic composition; per mil, parts per thousand; samples collected by M. Dailer and D. White and prepared by Dailer; analysis by U.S Geological Survey Reston Stable Isotope Laboratory using Schedule 2893]

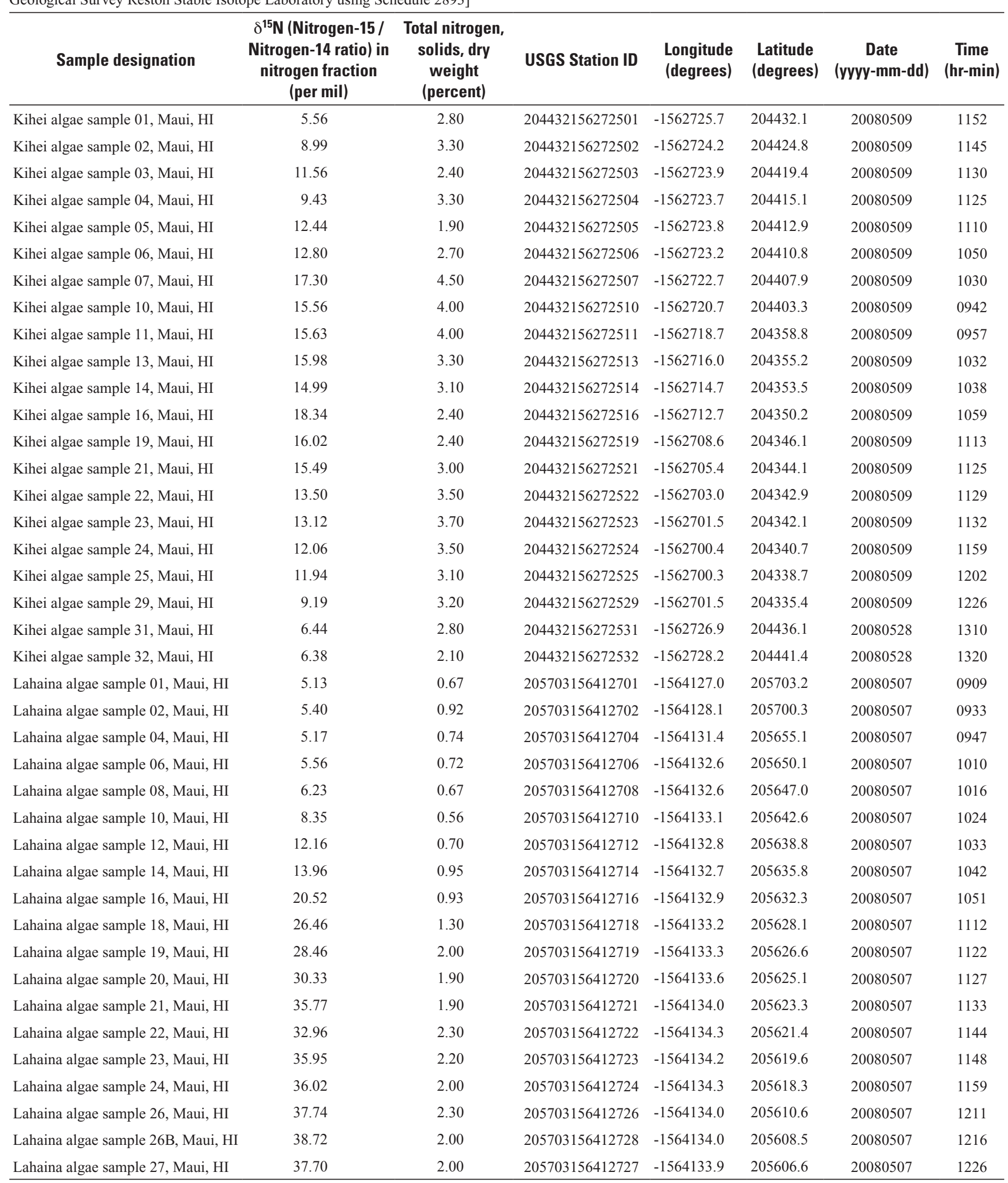


iced cooler. Algae samples were dried and ground to powder by Dailer, transferred into scintillation vials, and shipped to USGS for analysis of $\delta^{15} \mathrm{~N}$ isotopic composition and nitrogen concentration (mass fraction by dry weight) in algal tissue. Dailer provided GPS fixes for each sample.

\section{Detection and Mapping of Injected Effluent and Groundwater Discharge}

The survey methodology was able to detect the Kihei and Lahaina wastewater injection plumes where they discharge from aquifer to ocean, and this is demonstrated perhaps most convincingly using color-coded maps of the data. Selected maps guide the interpretive discussion that follows below. Biochemical processes apparently at work within the plumes are discussed here in a mapping context and in a more detailed discussion using mixing plots and computations in the section of the report "Mixing and Evolution of Injected Effluent." Complete map folios of all results are included in appendix A (trolling instrument surveys), appendix B (water and algae samples, Kihei), and appendix $\mathrm{C}$ (water and algae samples, Lahaina). Numeric concentrations and other values posted on the maps were rounded for ease of visual comparison.

\section{Trolling Instrument Surveys}

Trolling instrument surveys were least effective for inferring wastewater presence or nutrient sources, but they were successful in identifying fresher zones of groundwater discharge and provided a more continuous spatial pattern than possible with discrete samples. Kayak surveys were quicker and allowed multiple transects farther offshore, but wading surveys were better for sensing visual and temperature contrasts in the water, and prominent submarine springs at Kihei might have garnered less attention had we not waded and only surveyed by kayak instead.

Salinity and specific conductance were the most straightforward parameters to interpret, revealing low-salinity groundwater and/or effluent anomalies (fig. 15 and fig. 16, with salinity converted to equivalent freshwater fraction). Both effluent and upland groundwater are low in salinity (equivalent to about 1-2 percent seawater) and contrast strongly with seawater where discharge is focused. At Kihei (fig. 15), freshwater fractions as great as 25-49 percent (in red) were detected at a submarine spring and at a pool in the breakwall (shoreline rock revetment) where discharging groundwater is relatively protected against seawater mixing. Both zones are within the modeled extent of the injection plume, where discharge would be expected to be mostly effluent. At Lahaina (fig. 16), water was close to seawater salinity along most of the main beach, except near the submarine springs, where instrument readings indicated water as fresh as 4-10 percent freshwater fraction.
Water in the semienclosed south lagoon at Black Rock was much fresher (24-75 percent fresh) than water along the main beach.

Other instrument parameters varied with daylight and biotic cycles, and their meaning is somewhat equivocal. Water temperature (appendix A, figs. A3-A4, A13-A14) mostly reflected daytime heating over the duration of each survey from early morning starting times, particularly for the slower wading surveys. Injected effluent should be warmer than groundwater outside the plume and, notably, the submarine springs at Kahekili Beach near Lahaina were first described to us as "warm seeps." Table 3 shows that Kihei effluent $\left(27.5^{\circ} \mathrm{C}\right)$ was $7^{\circ} \mathrm{C}$ warmer than regional groundwater $\left(20.5^{\circ} \mathrm{C}\right.$ at the upgradient well). Effluent originates as tap water and is warmed at several stages: in households (hotwater bathing, dishwashing, and laundering), during sewer transmission (beneath hot roadways), and during wastewater treatment (in ponds exposed to sunlight). Although effluent may have good thermal contrast against ambient groundwater, it is close in temperature to nearshore seawater, and so temperature is not a good effluent tracer as a sole parameter. One can query the trolling datasets for a combination of high temperature and low salinity as a joint instrumental tracer of effluent, but a better course would be to rerun the surveys at a time of lesser thermal interference, earlier in the morning before the sun rises.

Dissolved oxygen and $\mathrm{pH}$ are known to vary with the daylight cycle. During the day, both increase as plant photosynthesis consumes carbon dioxide and produces oxygen. During the night, both decrease as photosynthesis ceases and animal respiration consumes oxygen and produces carbon dioxide, which lowers pH (Schwarz, 2006; Halley and Yates, 2008). Trolling surveys generally reflected this cycle, with the first early-morning transect of the day closest to shore having lower $\mathrm{pH}$ and dissolved oxygen than later transects farther offshore. Despite the general trends, there still appears to be some amount of "effluent-plume signature" in the dissolved oxygen and $\mathrm{pH}$ data. Dissolved oxygen at Kihei was lowest at the submarine spring and breakwater pool (appendix A, fig. A5) despite the overall increasing trend as the wading survey progressed from south to north at Kalama Park and then doubled back (the smaller survey segment at Waipuilani Park was much later in the day, about noon, resulting in much higher dissolved oxygen). A similar low-oxygen reach at Lahaina (appendix A, fig. A15) stretches north from the submarine springs but breaks abruptly to the south to much higher values (here, the kayak survey was from north to south before doubling back). Low dissolved oxygen was measured in the Kihei injection plume (table 3, downgradient well) and is expected in wastewater plumes generally, where bacterial metabolism of organic carbon consumes oxygen and produces anoxic conditions (LeBlanc, 1984).

Water $\mathrm{pH}$ displayed patterns similar to dissolved oxygen. At Kihei (appendix A, figs. A6-A7), a long reach of low pH spans much of the modeled injection plume, particularly the northern part where dissolved oxygen was lowest. At Lahaina 


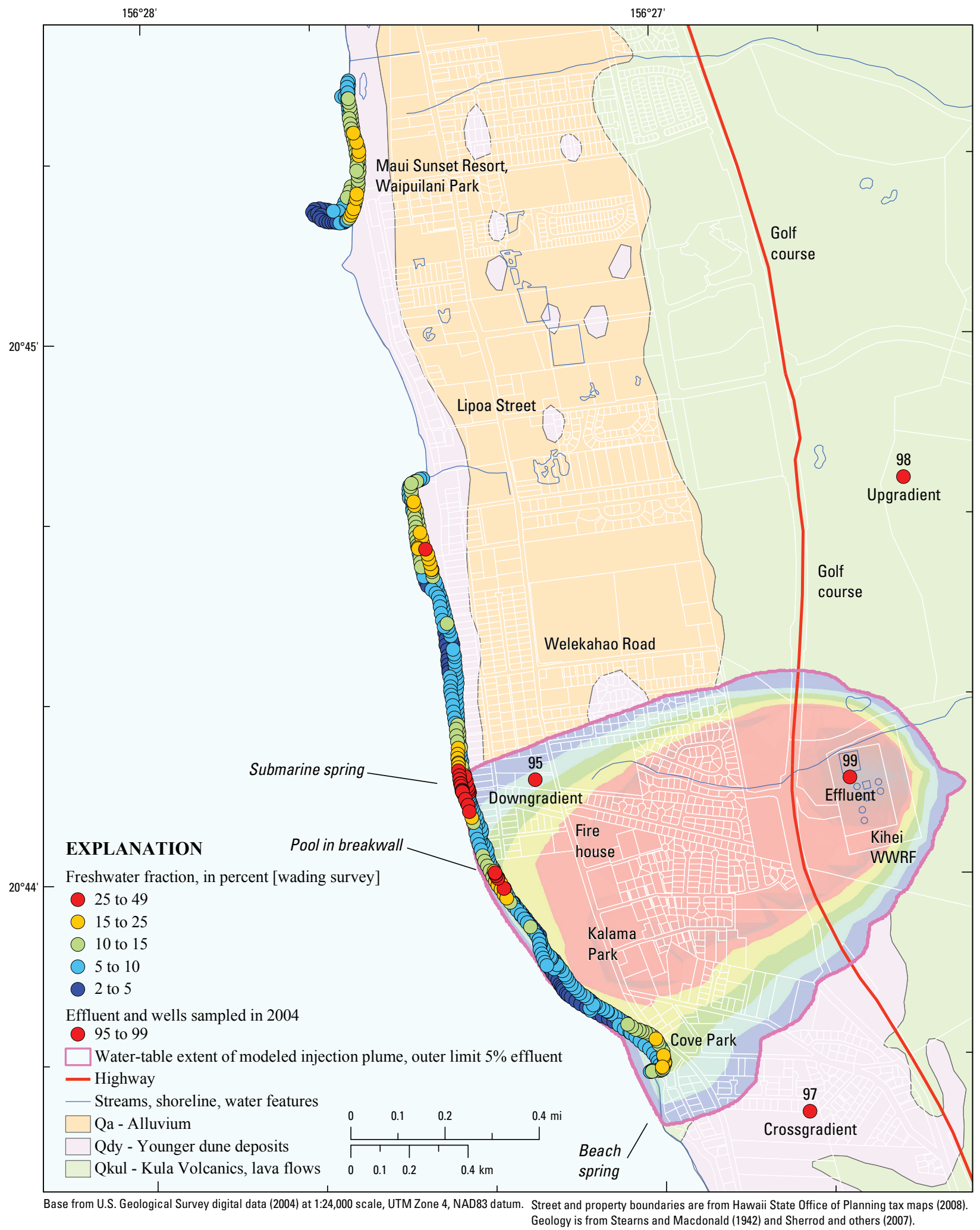

Figure 15. Map showing freshwater fraction along wading instrument survey at Kihei, Hawaii. 


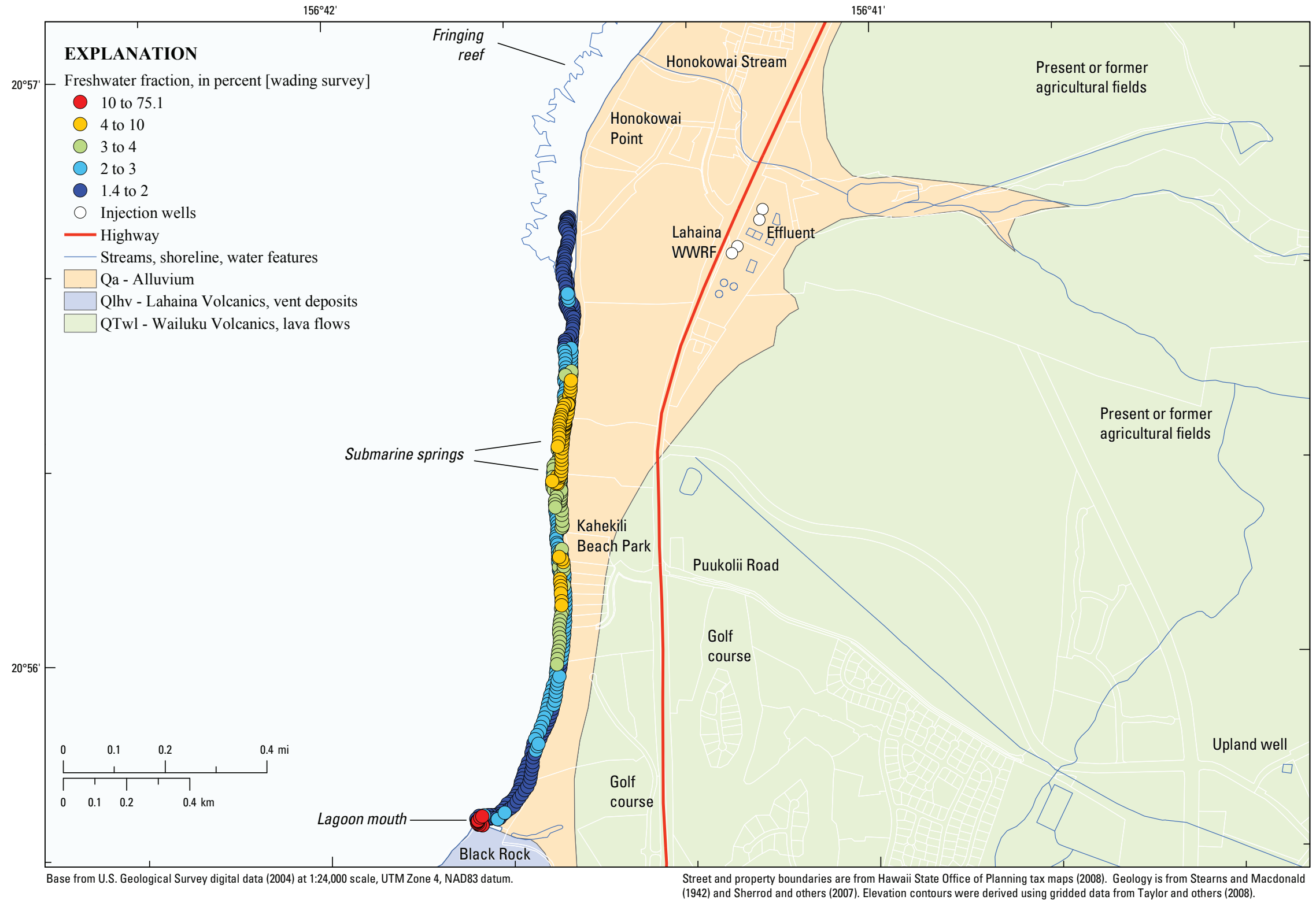

Figure 16. Map showing freshwater fraction along wading instrument survey at Lahaina, Hawaii. 
(appendix A, figs. A16-A17), a reach of low $\mathrm{pH}$ stretches north from the submarine springs but breaks abruptly to the south to higher values, much the same as the dissolved oxygen pattern. Lowest $\mathrm{pH}$ values were at Honokowai Point at the very start of the survey. Notably, operators at the wastewater reclamation facilities adjust the $\mathrm{pH}$ of effluent to near-neutral $(\mathrm{pH}=7)$, whereas seawater tends to maintain a $\mathrm{pH}$ near 8 as a result of natural buffering; effluent may therefore present a low-pH target for detection.

Chlorophyll and turbidity values were more erratic than other parameters but tended to be highest along the most inshore transect of the surveys (appendix A, figs. A8, A9, A18). This may reflect more phytoplankton inshore taking up groundwater-borne nutrients, or it could be a general artifact of wave-stirred sediment in shallowest waters. Chlorophyll and turbidity were both high in the lagoon at Black Rock and can be linked confidently there to the visible, bright-green phytoplankton bloom that appears to be a persistent feature of that water body (it is visible on several sets of air photos as well as being observed firsthand during our surveys).

Taken overall, instrument trolling (1) did a good job of detecting fresher groundwater discharge and helping to prioritize later water sampling; (2) allowed a modest degree of "plume-signature" inference by interpreting salinity, dissolved oxygen, and $\mathrm{pH}$ jointly; but (3) was ineffective for plume delineation without confirmation by chemical analysis. The patterns described did, however, turn out to correspond with chemical evidence of effluent discharge, as will be shown next.

\section{Water and Algae Samples}

Water-column and benthic algae samples were highly diagnostic of wastewater presence as a result of the low detection levels of laboratory analyses and the wastewater-specific nature of compounds or isotopes analyzed. Collection of laboratory water samples was guided by instrument trolling and a set of quick-reconnaissance water samples measured for salinity and fabric brightener fluorescence. Results of analyses and field measurements on water samples were tabulated in table 2 to table 6 and algae results in table 7 . As discussed earlier in the report, marine-water samples vary widely in degree of seawater dilution, and so constituent concentrations cannot be taken at face value without "unmixing" to source concentrations (table 3 and table 4 ) or the use of mixing plots (presented later in the report section "Mixing and Evolution of Injected Effluent").

\section{Salinity}

Reconnaissance sampling captured marine waters as much as 38 percent fresh at Kihei (appendix B, fig. B4) and 35 percent fresh at Lahaina (appendix C, fig. C4); additionally, a water sample from the lagoon at Black Rock was 91 percent fresh). Followup sampling for laboratory analysis at the same sites captured marine waters with maximum freshwater fractions of 52 percent at Kihei (appendix B, fig. B5) and 26 percent at Lahaina (appendix C, fig. C5); the lagoon sample measured 93 percent fresh. The strength of various wastewater tracers tended to relate directly to salinity, with strongest wastewater signatures in the freshest samples - given that samples were within the plume footprint in the first place.

\section{Fabric Brightener Fluorescence}

Fabric brightener fluorescence was measured on both reconnaissance and laboratory water samples (table 2 to table 4). The intent of this measurement was to detect optical brighteners (added to laundry detergents and also known as "fluorescent whitening agents" or "FWAs") as a means of inferring wastewater presence by its laundry graywater component. This emulates the approach of Hagedorn and others (2005), who successfully used it as a wastewater source tracking method in estuaries, in some cases tracing offshore detections back to failing septic systems onshore. The handheld fluorometer used in our surveys has a narrow-band response centered at $445 \mathrm{~nm}$ and spanning the emission wavelengths of optical brighteners (Turner Designs, 2007b; and meter operating manual and specifications). The meter was operated at the factory calibration, without calibrating to prepared brightener or detergent standards, and simply read as arbitrary meter fluorescence units.

It is important, however, to recognize that meter response may not be exclusively indicative of brightener presence. Natural dissolved organic matter (DOM) can be a source of interference, because its fluorescence spectrum overlaps the optical brightener band (Turner Designs, 2007a). Highly colored waters, such as brownish swamp water, owe their color to humic and fulvic DOM compounds, and humic acids in particular fluoresce in a similar excitation-emission band as optical brighteners (Chen and others, 2003). A high reading of 28 on the handheld meter was obtained on highly colored back-beach swamp water at Kahana Bay, Oahu, during the proof-of-concept phase of this project in 2006. Wastewater effluent is high in DOM (note the high organic carbon concentration in Kihei effluent, table 3, $3.9 \mathrm{mg} / \mathrm{L}$ ) and contains both humic-type fluorescence that overlaps the optical brightener band and protein-type fluorescence that does not (Henderson and others, 2009). Fluorescence readings in effluent using the handheld meter may therefore reflect contributions from (1) optical brighteners, (2) effluent humic DOM, or (3) both. Either way, an effluent fraction will give elevated readings, but an elevated reading does not necessarily confirm brighteners, as DOM could be the cause. Finally, whitening agents are not used solely in detergents but also in paper products, plastics (including styrofoam cups), and other products.

Fabric brightener fluorescence at Kihei was highest in lowest salinity waters within the plume footprint (appendix B, figs. B6, B7) but also high at Waipuilani Park far to the north, in waters no more than 17 percent fresh. At Lahaina, treated wastewater effluent had a reading of 31.7, whereas highest readings in environmental waters ranged from 2.9 to 4.4 at 
the submarine springs and lagoon at Black Rock (appendix C, figs. C6, C7), with intermediate values of 0.8 to 1.0 at the upland well and far north marine sample L4 just off Honokowai Stream mouth. A deionized-water reference sample registered 0.002 and tap water read 0.125 .

Fluorescence had only modest value for wastewater detection. High values were obtained where effluent was expected at Kihei (appendix B, figs. B6, B7), but intermediate to high values also occurred outside the plume footprint. The significance of high readings at Waipuilani Park is unclear; the high readings may indicate fabric brighteners are present, but there was no confirmation by any other wastewater tracers, suggesting that natural humic compounds may be responsible. At Lahaina (appendix C, figs. C6, C7), high readings in the lagoon at Black Rock probably are due largely to DOM compounds (the water is highly colored), although isotopic composition and mixing calculations (shown later) suggest a possible effluent fraction of 32 percent there. Similarly, the moderately elevated value of 1.0 at the far north site L4 was not strongly supported by other wastewater tracers and may be related to humic compounds, possibly in the impounded stream backwater seeping through the beach berm. Greater confidence might have been possible had we applied methods to discriminate brightener from nonbrightener fluorescence by UV illumination (Hartel and others, 2007; Cao and others, 2009). Another improvement would be a fabric brightener sensor on the multiparameter sonde to obtain continuous trolling data that might better show patterns.

\section{Pharmaceuticals and Waste Indicator Compounds}

Pharmaceuticals and waste indicator compounds were highly diagnostic of effluent presence, but because they are costly analyses, only 4 samples per locale were run for pharmaceuticals and 1 sample per locale for waste indicators (table 5 and table 6). Fewer compounds were detected in environmental samples than in treated wastewater effluent as a result of (1) effluent dilution with groundwater, (2) sorption of compounds in the mixed effluent and groundwater plume by soil and organic matter, and (3) biological and chemical degradation of compounds within the mixed effluent and groundwater plume.

At Kihei (fig. 17 and table 5), the pharmaceutical carbamazepine was detected in all 3 water-column samples within the modeled plume footprint, caffeine was detected in 2 of those samples, and sulfamethoxazole in 1 sample. No pharmaceuticals were detected at Waipuilani Park. Carbamazepine also was detected in treated effluent and the downgradient well in 2004, but sulfamethoxazole and caffeine were not, possibly as a result of varying removal efficiency in treatment from day to day and the fact that samples are not cosynchronous (an effluent fraction being detected in the environment was injected at some past and unknowable time that differs from sample to sample). Other detections in the 2004 effluent were cotinine (a nicotine metabolite), diphenhydramine (an antihistamine), and trimethoprim (an antibiotic).

At Lahaina (fig. 18 and table 6), carbamazepine and sulfamethoxazole were detected in effluent and at both submarine springs but not in the more northerly samples along Honokowai Point. Diphenhydramine also was detected at one spring but not in effluent, again possibly owing to time-varying removal efficiency during treatment. Caffeine was detected in both of the Honokowai Point samples, and one of them also contained 1,7-dimethylxanthine, a caffeine metabolite. Notably, however, carbamazepine and sulfamethoxazole were not detected in those samples, and effluent sampled that day did not contain detectable caffeine or the metabolite.

Carbamazepine is an anticonvulsant and antiepileptic drug detected frequently in sewage effluents and environmental waters containing effluent (Focazio and others, 2008). It is resistant to degradation (recalcitrant) during wastewater treatment and persists during offsite transport more than many other compounds (Drewes and others, 2003; Glassmeyer and others, 2005). Sulfamethoxazole is an antibiotic that also is persistent through waste treatment and readily transported with little retardation (Barber and others, 2008). Both carbamazepine and sulfamethoxazole were detected in treated wastewater effluent and at offsite wells and springs at Kealakehe, Hawaii (Hunt, 2008). Their detection in this study is a strong indication of treated effluent presence within the modeled plume footprint at Kihei and at the submarine springs at Lahaina. The lack of detectable pharmaceuticals at Waipuilani Park argues against wastewater presence there, despite high fabric brightener fluorescence readings.

Caffeine is a stimulant familiar to most by its content in coffee, soft drinks, and "energy" beverages. Nondetection of caffeine in both the Kihei and Lahaina effluents suggests high removal efficiency during sewage treatment, or at least varying efficiency such that it was not detected in effluent on the day sampled. Caffeine is known to have high removal efficiency in many wastewater treatment plants (Buerge and others, 2006), particularly those employing activated sludge biologic treatment, as is the case at Kihei and Lahaina. At Honokowai Point, detection of caffeine and its metabolite — but nondetection of the highly persistent carbamazepine and sulfamethoxazolecould conceivably have resulted from time-varying removal of the various compounds. But this unusual combination of detections and nondetections suggests other hypotheses. One hypothesis is that caffeine and its metabolite were excreted locally by recreational swimmers, because there are large hotels on the beach and the ocean is often crowded with visiting tourists as well as local residents who also enjoy the beach and are attracted to its popular surf spot. An alternate hypothesis is that caffeine and its metabolite indicate chronically leaking sewer lines in the vicinity or a holdover from past storm-related sewer overflows. Our May 2008 surveys were conducted in fair weather, but heavy rains 6 months earlier in December 2007 may have caused sewer overflows at that time, and infiltration and later slow drainage of the surficial 


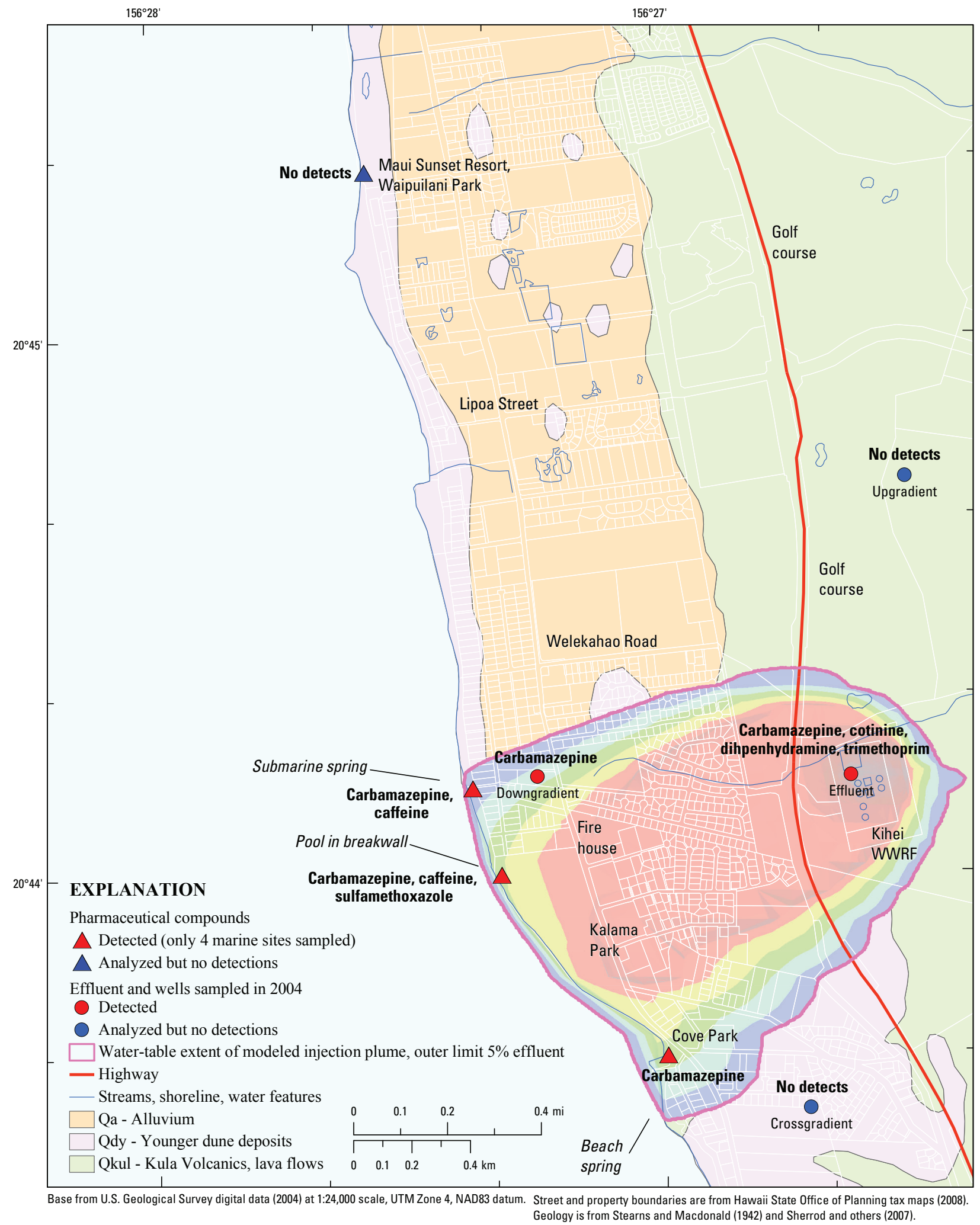

Figure 17. Map showing pharmaceuticals in water at Kihei, Hawaii. 


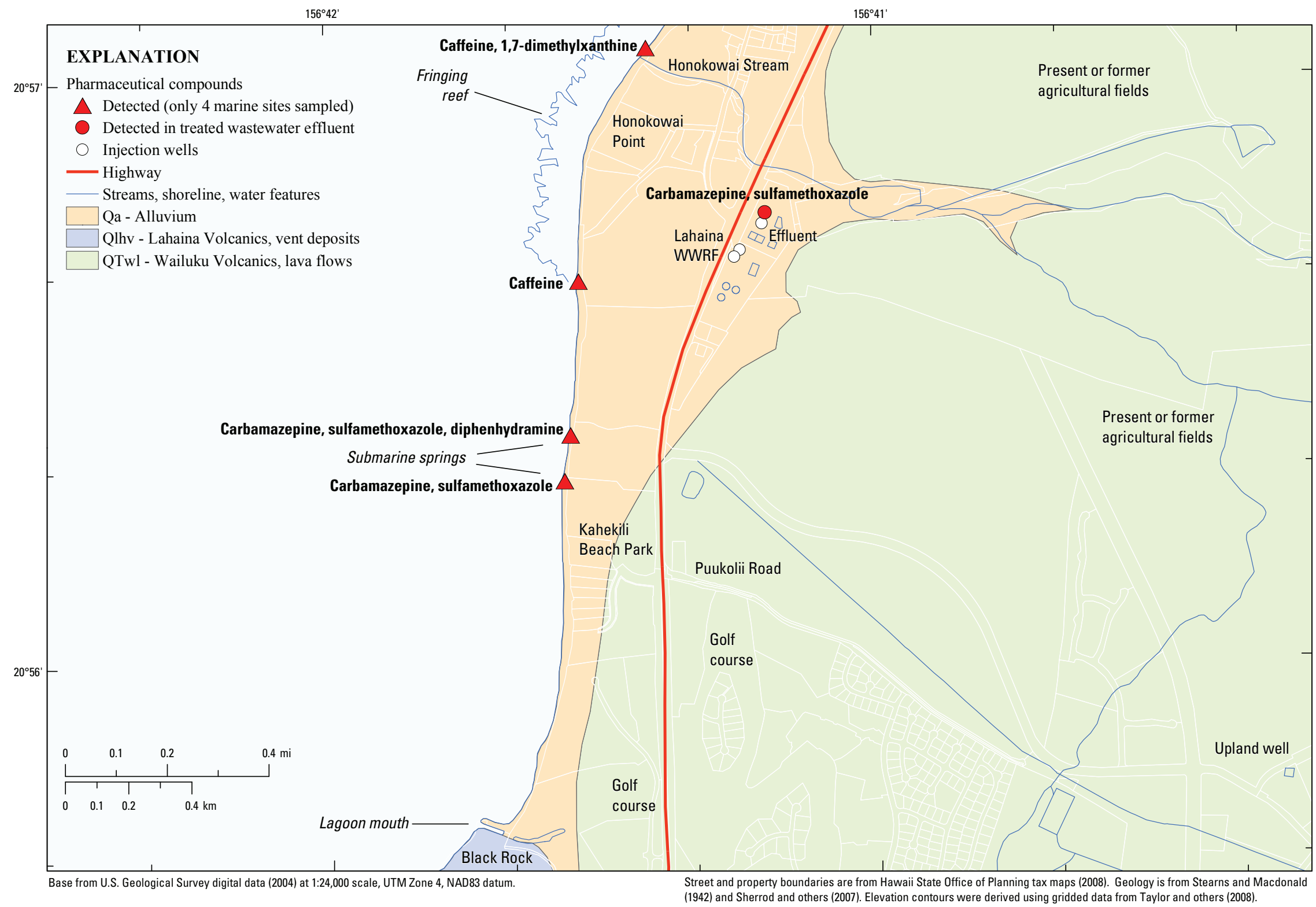

Figure 18. Map showing pharmaceuticals in water at Lahaina, Hawaii. 


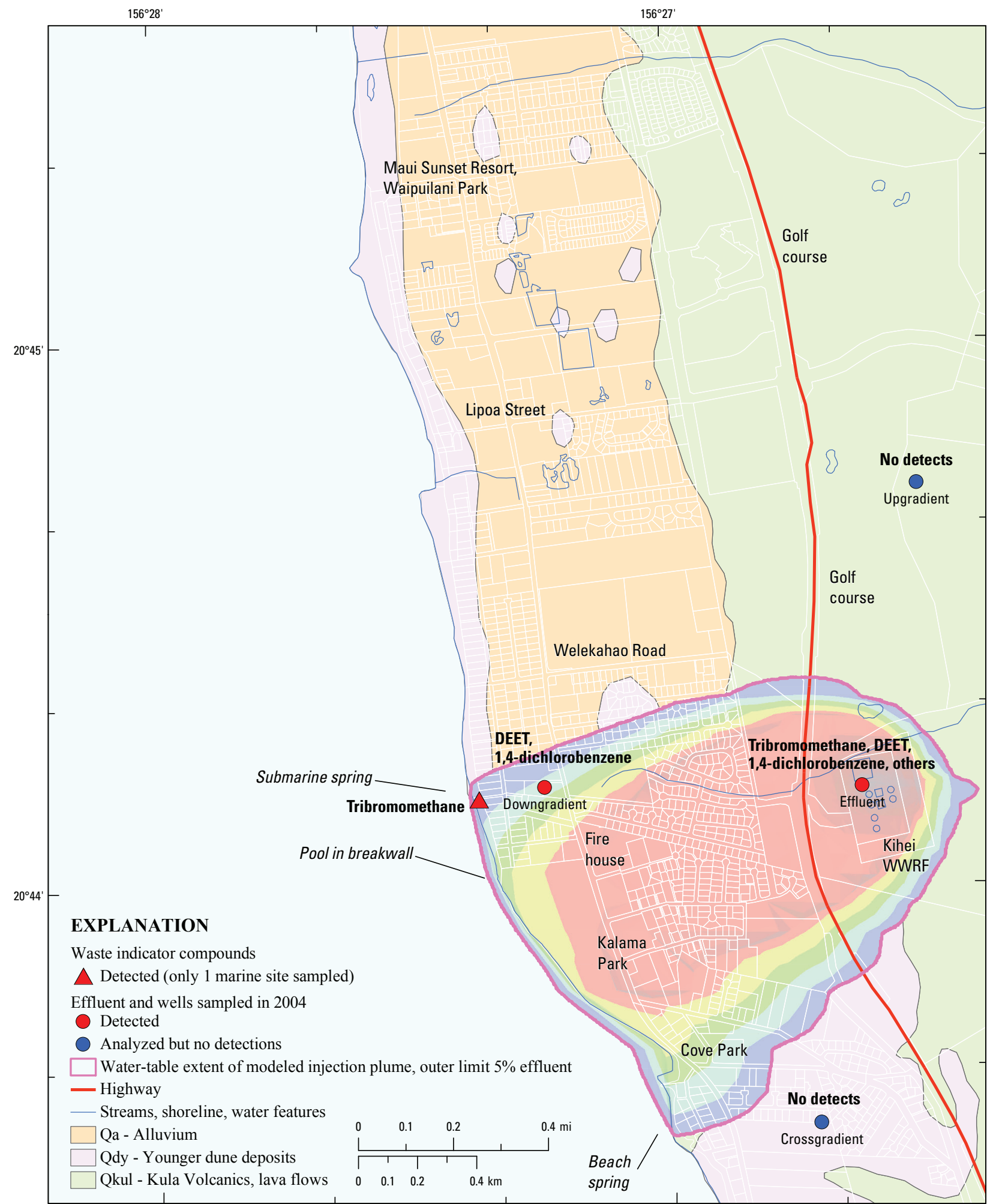

Base from U.S. Geological Survey digital data (2004) at 1:24,000 scale, UTM Zone 4, NAD83 datum. Street and property boundaries are from Hawaii State 0ffice of Planning tax maps (2008). Geology is from Stearns and Macdonald (1942) and Sherrod and others (2007).

Figure 19. Map showing waste indicator compounds in water at Kihei, Hawaii. 
landscape may offer a mechanism for slow release of waste contaminants into the fair-weather season. Several workers point to storm-related combined sewer overflows (CSOs) for detection of wastewater compounds with high removal efficiencies during waste treatment (Buerge and others, 2006; Phillips and Chalmers, 2009); it is the overflows of raw sewage that release these compounds before treatment, while stormwater dilution lowers concentrations of other compounds that have high persistence through treatment.

At Kihei, codetection of caffeine with carbamazepine and sulfamethoxazole (but with no caffeine detected in effluent) may simply reflect time-varying caffeine removal in treatment, but the above mechanisms of swimmers, chronically leaking sewer lines, and prior storm-caused sewer overflows also are possible. It may be that mixed sewage signals are being detected: treated and injected effluent carrying carbamazepine and sulfamethoxazole, plus chronic sewer leaks or tailing from prior overflows introducing caffeine. These multiple, confounding sewage sources could also include remnant cesspools, although both the Kihei and Honokowai areas are sewered, and a joint search of water and sewer accounts for the Kalama Park area of Kihei indicated no active cesspools remaining (Steve Parabicoli, Maui County, written commun., 2008). The likelihood of sewer collector-line leakage is unknown, but the tracers used in this study could offer a means for investigating that topic.

In the waste indicators sample at Kihei (fig. 19 and table 5), only tribromomethane (bromoform) was detected, a water disinfection byproduct that was also detected in Kihei effluent in 2004. Sample K1 was chosen for analysis because of its high freshwater fraction and the strength of the submarine spring there. In hindsight, sample K2 or K8 (fig. 9) might have made a better choice: they were nearly as fresh as sample K1 but are located more in the core of the plume and probably would have yielded additional detectable compounds.

Better results were obtained at Lahaina on the single waste indicators sample L1 (fig. 20 and table 6) in which five compounds detected also were detected in effluent: tribromomethane, two synthetic musk fragrances (AHTN and HHCB), a fire retardant tris(dichloroisopropyl) phosphate, and a plasticizer tris(2-butoxyethyl) phosphate. A number of additional compounds were detected in the Lahaina effluent, and the same musk fragrances, fire retardants, and plasticizers detected in Lahaina sample L1 were also detected in the 2004 Kihei effluent (table 5). The correspondence of five waste indicator compounds in Lahaina effluent and spring sample L1 is a strong indication of effluent at L1.

\section{Nutrients, Nutrient Transformations and Processes, and Nitrogen Stable Isotopes}

Nutrients are important in coastal ecology, and the high nutrient content of wastewater compared to receiving coastal waters raises potential concerns about eutrophication. Nitrogen $(\mathrm{N})$ and phosphorus $(\mathrm{P})$ can serve as supporting wastewater indicators at high concentrations, but they are not solely diagnostic of wastewater because fertilizer use can also increase these concentrations. Treated wastewater on Maui has higher nitrogen and phosphorus concentrations than most groundwater beneath fertilized agricultural fields. However, both effluent and agricultural groundwater contain more $\mathrm{N}$ and P than "background" groundwater beneath upland forests. In addition, all groundwater - even background - is several orders of magnitude higher in $\mathrm{N}$ and $\mathrm{P}$ concentration than seawater (Tetra Tech, 1993; Dollar and Andrews, 1997; Soicher and Peterson, 1997; Laws and others, 2004; Hunt, 2007; Smith and Smith, 2007). This is illustrated in fig. 21, which includes samples of Kihei and Lahaina effluents and other representative concentrations assigned mostly from water samples in this study (only the Lahaina forest background value of 21 is from Soicher and Peterson, 1997). Cesspools and septic systems are additional sources of waste nutrients, as are pets and animals at pasture or inhabiting the watershed. Nitrogen also is fixed naturally from the atmosphere by soil bacteria, and phosphorus is derived from rock weathering. Nitrogen and phosphorus dissolve in infiltrating water and are transformed through biotic cycles such as organic-matter decomposition and uptake and cycling in the food chain.

Nitrogen is generally more mobile in groundwater than phosphorus, which tends to sorb strongly to soil and other earth materials. Figure 22 shows the concentration and speciation of nitrogen in single, one-time samples of Kihei and Lahaina effluent. Although the two samples are comparable in total nitrogen, they differ substantially in nitrogen speciation, with Lahaina having a larger proportion of its nitrogen in ammonia form than Kihei. These relations could be characteristic of the effluents generally, resulting from the particular types and sequence of treatment processes at each plant. Alternatively, the patterns in fig. 22 could simply be instantaneous snapshots within a wide range of variation, and repeat sampling for the various nitrogen species would be required to characterize that variation. Inspection of paper records at the Kihei plant in 2004 indicated that total nitrogen was quite stable from day to day over periods of months at about 7 milligrams per liter as $\mathrm{N}$ (500 micromoles per liter), suggesting a high degree of plant stability that likely results from the tertiary level of treatment and competent operation.

Once injected into the basaltic aquifers at Kihei and Lahaina, the effluents are subject to microbial consumption and transformation of the organic matter and nutrients they contain. Spatial zonation will develop within the plumes, depending on such factors as the supply of oxygen and other electron acceptors and donors that regulate microbially mediated oxidation-reduction (redox) reactions. From studies at Cape Cod and elsewhere, expected nitrogen transformations include loss of ammonia by volatilization and by nitrification to nitrate where oxygen is present; consumption of organic carbon and oxygen by bacterial respiration, resulting in a suboxic to anoxic core of the plume where nitrate is denitrified to $\mathrm{N}_{2}$ and $\mathrm{N}_{2} \mathrm{O}$ gases and where ammonium is stable although retarded by sorption; anaerobic loss of ammonium through the 


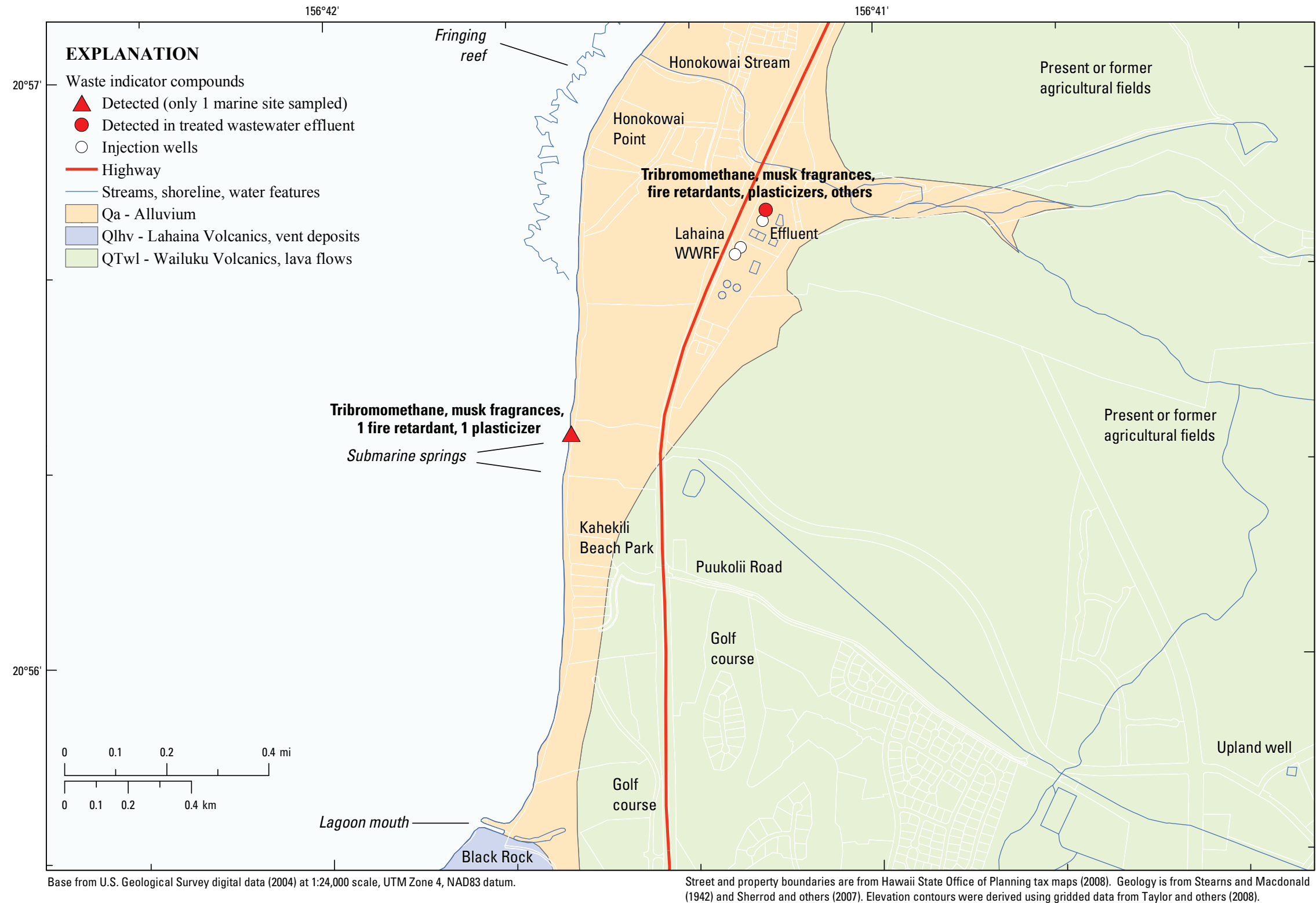

Figure 20. Map showing waste indicator compounds in water at Lahaina, Hawaii. 


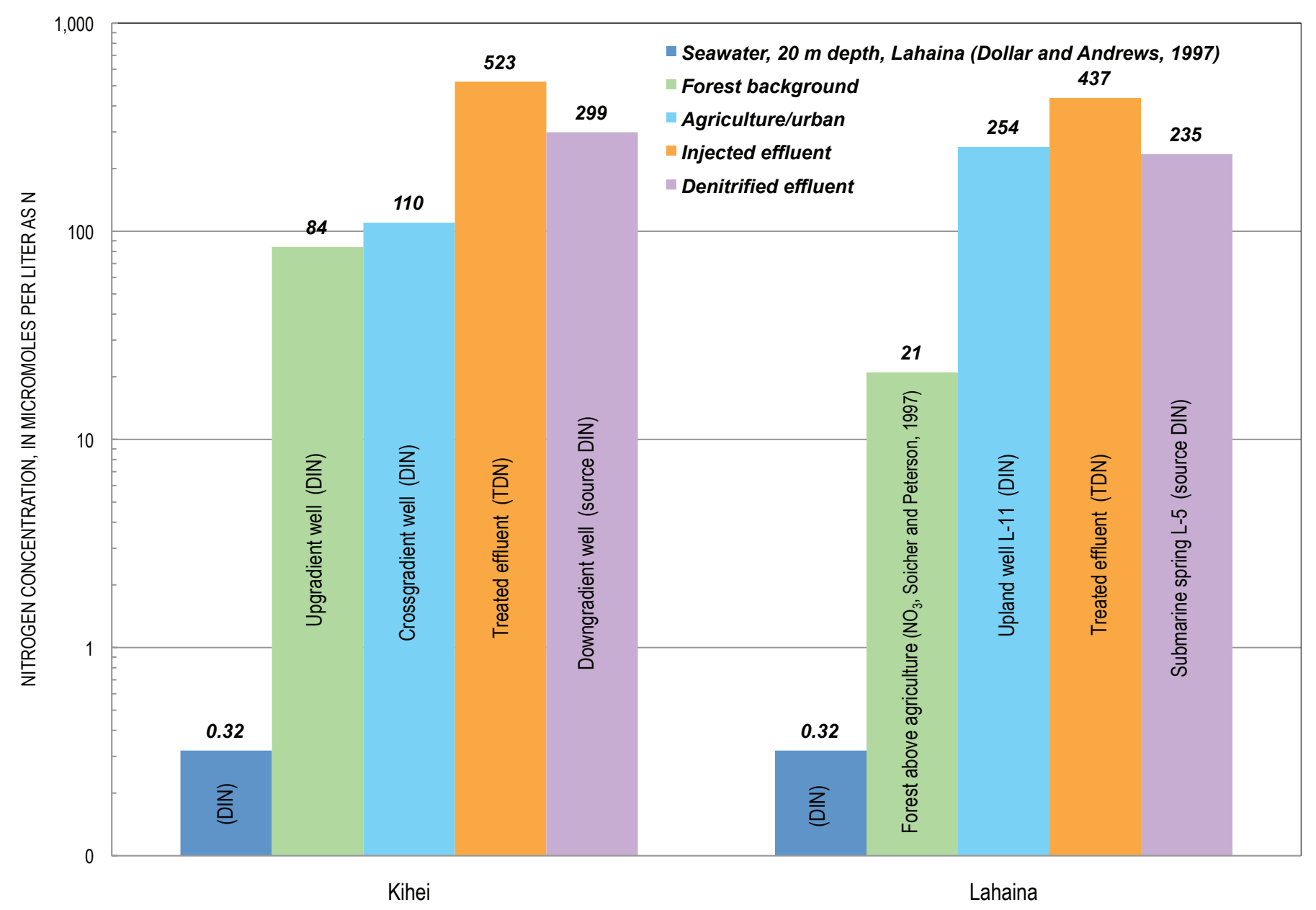

Figure 21. Graph showing nitrogen concentrations associated with different land uses and nitrogen sources, using data mostly from this study.

annamox process; and ammonification of organic nitrogen to ammonium (some of these processes are illustrated on figure 23, from Bohlke and others, 2006). Phosphorus transport will also be governed by geochemical conditions, with a tendency for sorption and incorporation into mineral phases.

The various reactions will tend to cause plume zonation into concentric shells or halos, for example from a suboxicanoxic core to an outer halo where oxygen is again introduced by regional groundwater flow mixing in at the lateral margins of the plume. Further complications include the possibility of microzones where contrasting conditions (for example oxic and anoxic) can exist in close proximity. Given that our surveys are essentially transverse sections across the plumes, the data in our study are likely to reflect spatial variation in geochemical conditions across the shells or halos of the plumes as well as simple dilution gradients from plume to ambient groundwater and saltwater at the margins. The various processes are complex enough that they cannot be characterized from the very limited data of this reconnaissance-level study. At best, we may be able to observe in our data isotopic shifts or nutrient patterns that hint at processes taking place in the Maui plumes.
Stable-isotope compositions are modified by fractionation, in which a physical, chemical, or biologic process selectively incorporates the lighter or heavier isotope and leaves behind a residual pool of material enriched in the other isotope. Nitrogen isotopic values can be set or reset during nitrogen-cycling processes such as those described above. The useful isotope classification in figure 24 has evolved and has been used to infer (1) the source of nitrogen in a given environmental sample - for example, on the strength of $\delta^{15} \mathrm{~N}$ being heavier in animal/human waste nitrogen than in soil nitrogen or fertilizers; and (2) processes modifying sample composition - for example, denitrification, which drives $\delta^{15} \mathrm{~N}$ and $\delta^{18} \mathrm{O}$ compositions in nitrate toward heavier values from any particular source composition.

Source inference is best applied judiciously, however, because nitrogen isotopic composition does not remain fixed at its source value or behave conservatively (varying only by simple mixing) but instead is readily modified by processes such as those mentioned above. Although the intent may be to characterize nitrogen sources, one is actually sampling at environmental destinations. Environmental samples are not 


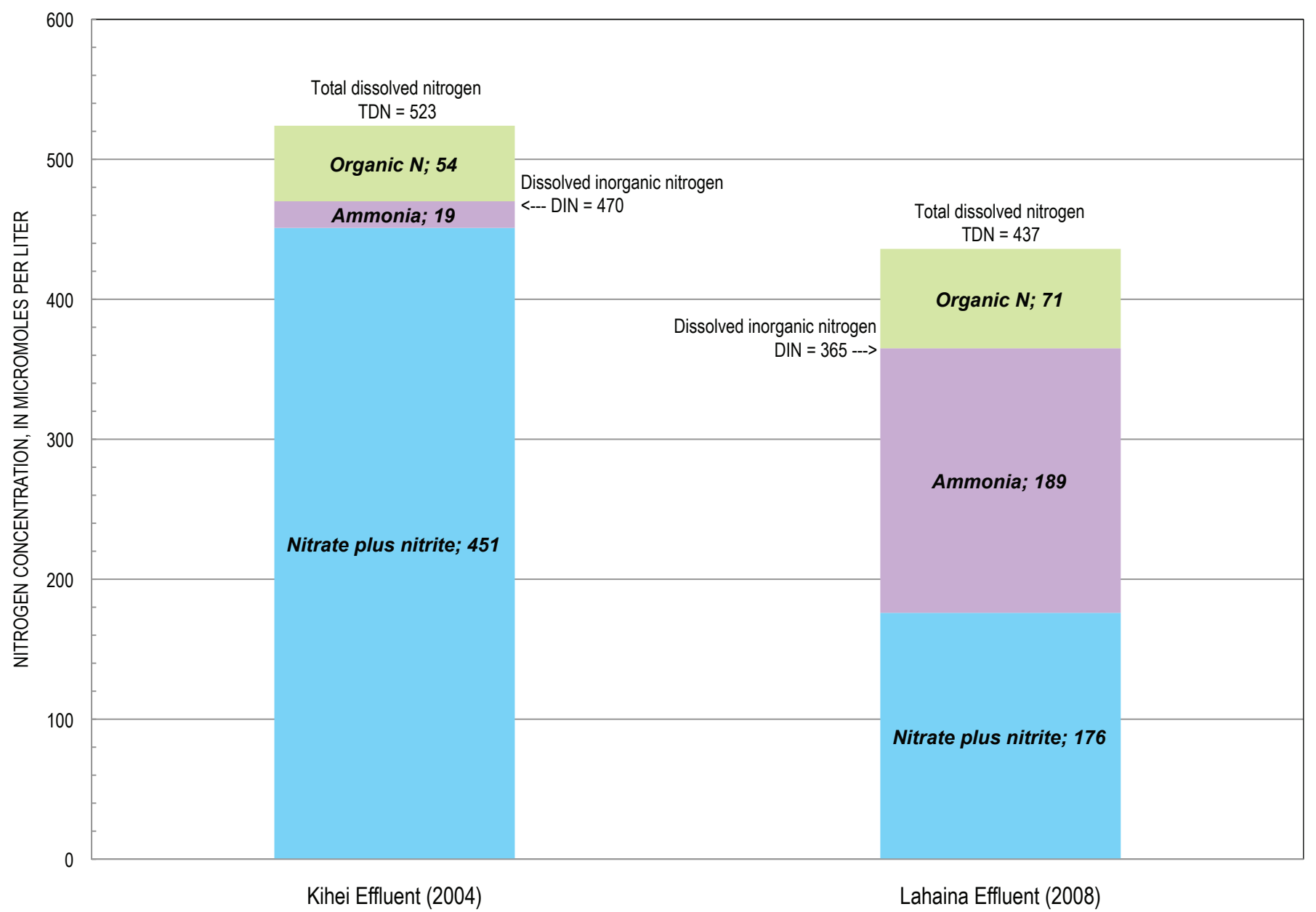

Figure 22. Graph showing nitrogen concentration and speciation in treated wastewater effluent from the Kihei and Lahaina Wastewater Reclamation Facilities.

taken at the source, and original source compositions may have been altered along the way. Using denitrification as an example, Kendall (chapter 16 in Kendall and McDonnell, 1998) cautions: "Denitrification causes the $\delta^{15} \mathrm{~N}$ of the residual nitrate to increase exponentially as nitrate concentrations decrease. For example, denitrification of fertilizer nitrate that originally had a distinctive $\delta^{15} \mathrm{~N}$ value of +0 per mil can yield residual nitrate with much higher $\delta^{15} \mathrm{~N}$ values (e.g. +15 to +30 per mil) that are within the range of compositions expected for nitrate from a manure or septic-tank source." This is illustrated by the field study of Mengis and others (1999), where groundwater beneath crops receiving urea and mineral fertilizers (no animal manure) had light $\delta^{15} \mathrm{~N}$ values of +3.8 to +10.1 per mil, whereas $\delta^{15} \mathrm{~N}$ values as great as +14.5 to +48.8 per mil were measured in groundwater beneath the downgradient riparian zone. The increased isotopic compositions and greatly diminished nitrate concentrations downgradient were consistent with denitrification of fertilizer nitrate along the groundwater flow path. Despite these complexities, nitrogen-isotope composition was a highly effective wastewater tracer in this study, equal to pharmaceuticals and waste indicator compounds in confirmation power and with good agreement between water and algae compositions. Most of the isotopic narrative that follows centers on denitrification, because there is strong evidence for it in the Maui plumes. Discussion is offered more as an example of data interpretation than a presumption of knowing exactly what is occurring in the aquifer. Isotopic compositions may be affected by other nitrogen processes, such as sorption and nitrification of ammonium, but it is much harder to infer their effects without process-oriented study of the plumes.

\section{Nutrients and Nitrogen Isotopes at Kihei}

At Kihei, $\delta^{15} \mathrm{~N}$ of algal-tissue nitrogen delineated the lateral extent of the effluent plume quite well, in part because the large number of algae samples approximated a continuous transect (fig. 25 and table 7). Heavy $\delta^{15} \mathrm{~N}$ values of +15 to +18 per mil were measured across the core of the modeled plume, falling off to values as light as +6 per mil outside the plume, not much different from the +5 per mil in dissolved nitrate at the upgradient and crossgradient wells. This pattern was 


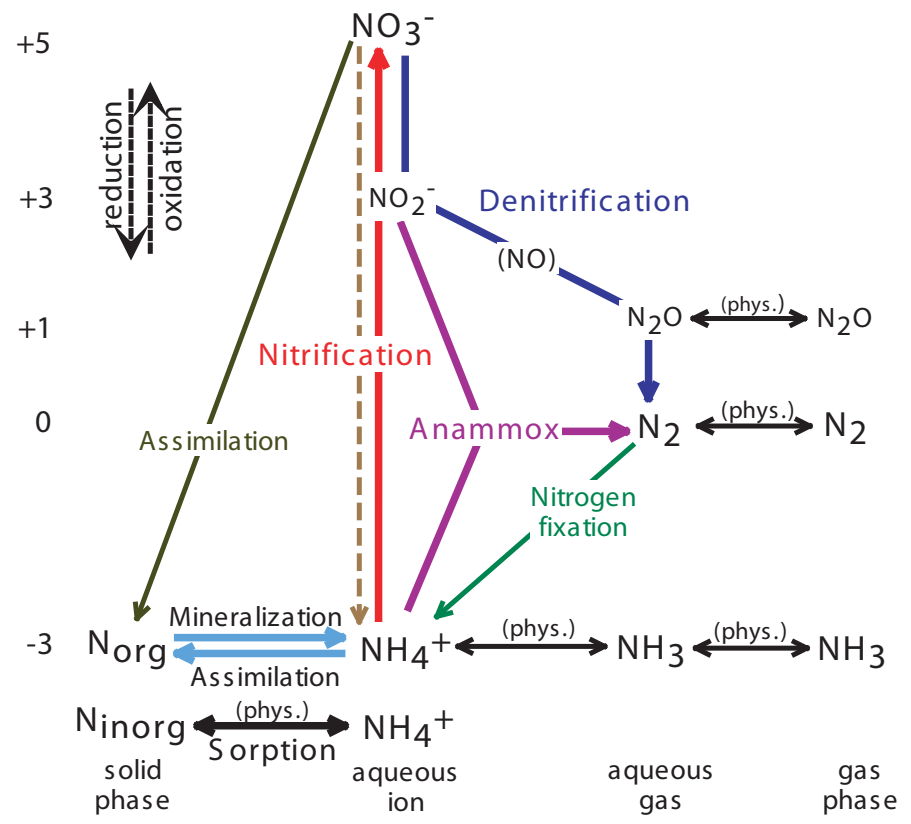

Figure 23. Diagram showing biogeochemical and physicalchemical processes affecting the speciation of nitrogen in aquatic systems (from Bohlke and others, 2006 and provided by J.K Bohlke, written commun., 2009).

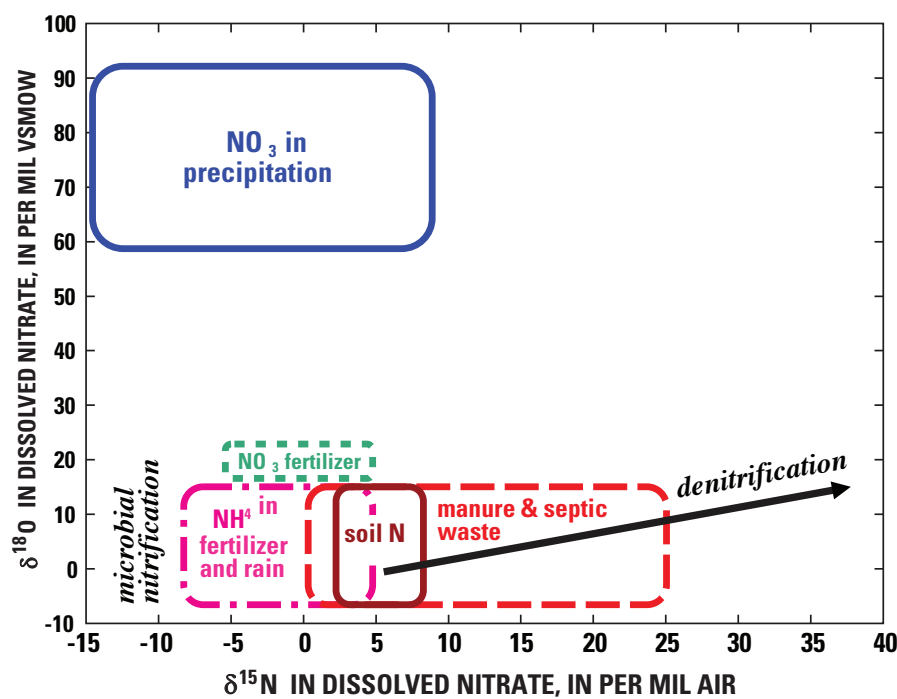

Figure 24. Graph showing nitrate source classification based on nitrogen and oxygen stable-isotope compositions of dissolved nitrate (modified from Kendall and McDonnell, 1998, by Carol Kendall, written commun., 2006). mimicked by nitrogen concentration by dry weight in algal tissue (appendix B, fig. B11). Algae within the effluent plume have incorporated more nitrogen, a sign that they are exposed to higher dissolved nitrogen concentrations there. Taken together, the heavy $\delta^{15} \mathrm{~N}$ and high nitrogen concentrations in algae are a strong indicator of wastewater presence.

Nitrogen isotopes in dissolved nitrate (fig. 26 and table 3) confirm the pattern in algae and provide direct comparability with wells and effluent sampled in 2004 by being measured in the same medium (water). The maximum $\delta^{15} \mathrm{~N}$ value of +18 per mil at the shore was the same as in algae, and water samples outside the probable influence of the plume had lighter $\delta^{15} \mathrm{~N}$ values of +6 to +8 per mil, also similar to values in outlying algae. Good agreement between water and algae compositions supports the proposition that the algae incorporate nitrogen with little discriminatory fractionation (for example Smith and Smith, 2007), providing a natural "accumulating sampler" of dissolved nitrogen that maintains its isotopic signature.

The maximum $\delta^{15} \mathrm{~N}$ of +18 per mil in algae and marine water at Kihei is only slightly heavier than the +15 per mil in Kihei effluent. This probably reflects slight enrichment in the heavy isotope by denitrification, although time variation in effluent source composition is also a possibility. Hunt (2007) proposed that the +23 per mil $\delta^{15} \mathrm{~N}$ in the downgradient well had evolved by denitrification of effluent in the aquifer under anoxic conditions. Seeing that no $\delta^{15} \mathrm{~N}$ that heavy was measured at the shore in this study, the downgradient well does not appear to be wholly representative of the rest of the plume and may reflect somewhat local conditions toward the lateral periphery of the plume, in carbonate sediments that overlie the basalt aquifer.. Intermediate $\delta^{15} \mathrm{~N}$ values of +11 to +14 per mil probably correspond to mixtures of effluent and upland groundwater, whereas lighter values ranging +6 to +9 per mil could reflect (1) slight denitrification from the upland composition of +5 per mil, (2) some small fraction of effluent mixed in with upland water, or (3) slight addition of nitrogen heavier isotopically than upland groundwater as that groundwater passes beneath the urban strip on its way to the shore. These possibilities cannot be distinguished solely on the basis of isotopic composition, and confirmation of a wastewater fraction in samples with light-to-intermediate $\delta^{15} \mathrm{~N}$ values requires support from another tracer, such as pharmaceuticals or waste indicator compounds. Dollar (1996) reported a "marine" $\delta^{15} \mathrm{~N}$ end-member composition of +3.7 per mil for benthic algae from Hawaiian ocean waters and a "land" end-member value of +8 per mil for algae from streams and freshwater seeps in North Kohala, Hawaii. Casciotti and others (2008) reported $\delta^{15} \mathrm{~N}$ of +1.5 to +2.4 per mil in dissolved nitrate at $150 \mathrm{~m}$ depth in the upper thermocline of open ocean water near Oahu, and values as heavy as +7.1 per mil in water below that.

Dissolved inorganic nitrogen (DIN) at Kihei was highest within and near the modeled effluent plume (fig. 27). Effluent DIN was $470 \mu \mathrm{M}$ and effluent TDN was $523 \mu \mathrm{M}$. TDN is shown because some organic $\mathrm{N}$ can be transformed and utilized in nitrogen cycling (Seitzinger and others, 2002), and so TDN may be a more relevant measure of the total available 


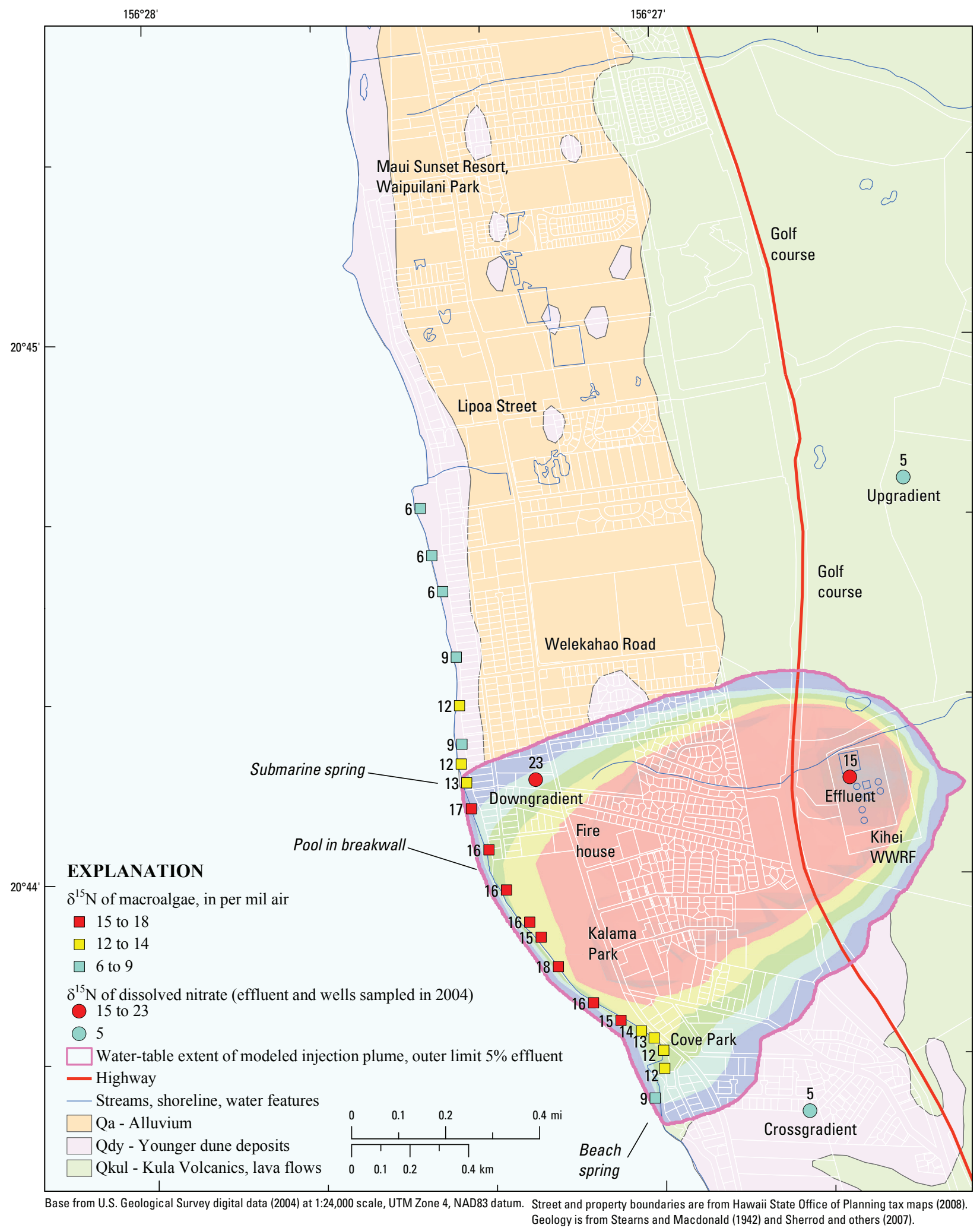

Figure 25. Map showing $\delta^{15} \mathrm{~N}$ isotopic composition of macroalgae at Kihei, Hawaii. 


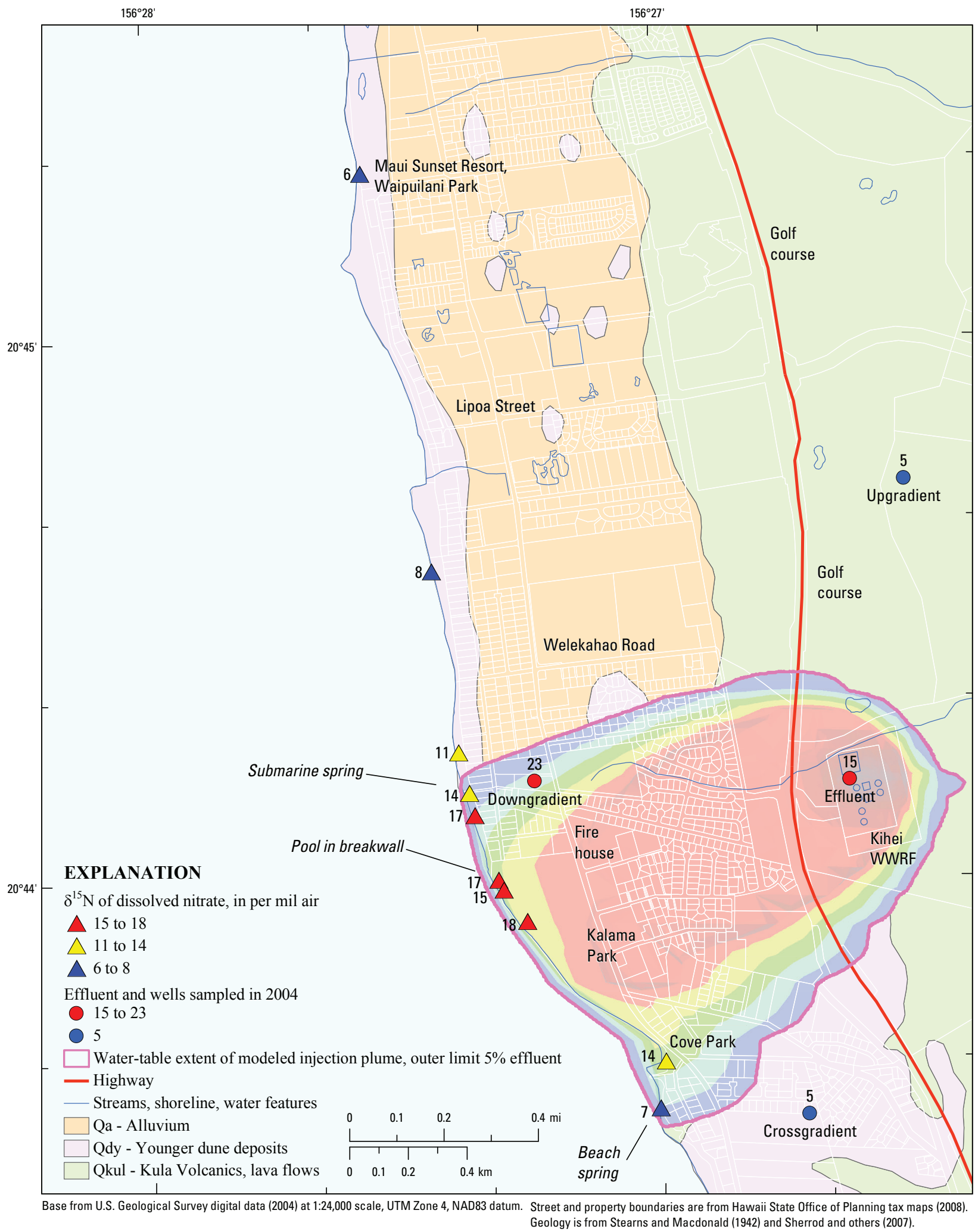

Figure 26. Map showing $\delta^{15} \mathrm{~N}$ isotopic composition of dissolved nitrate at Kihei, Hawaii. 


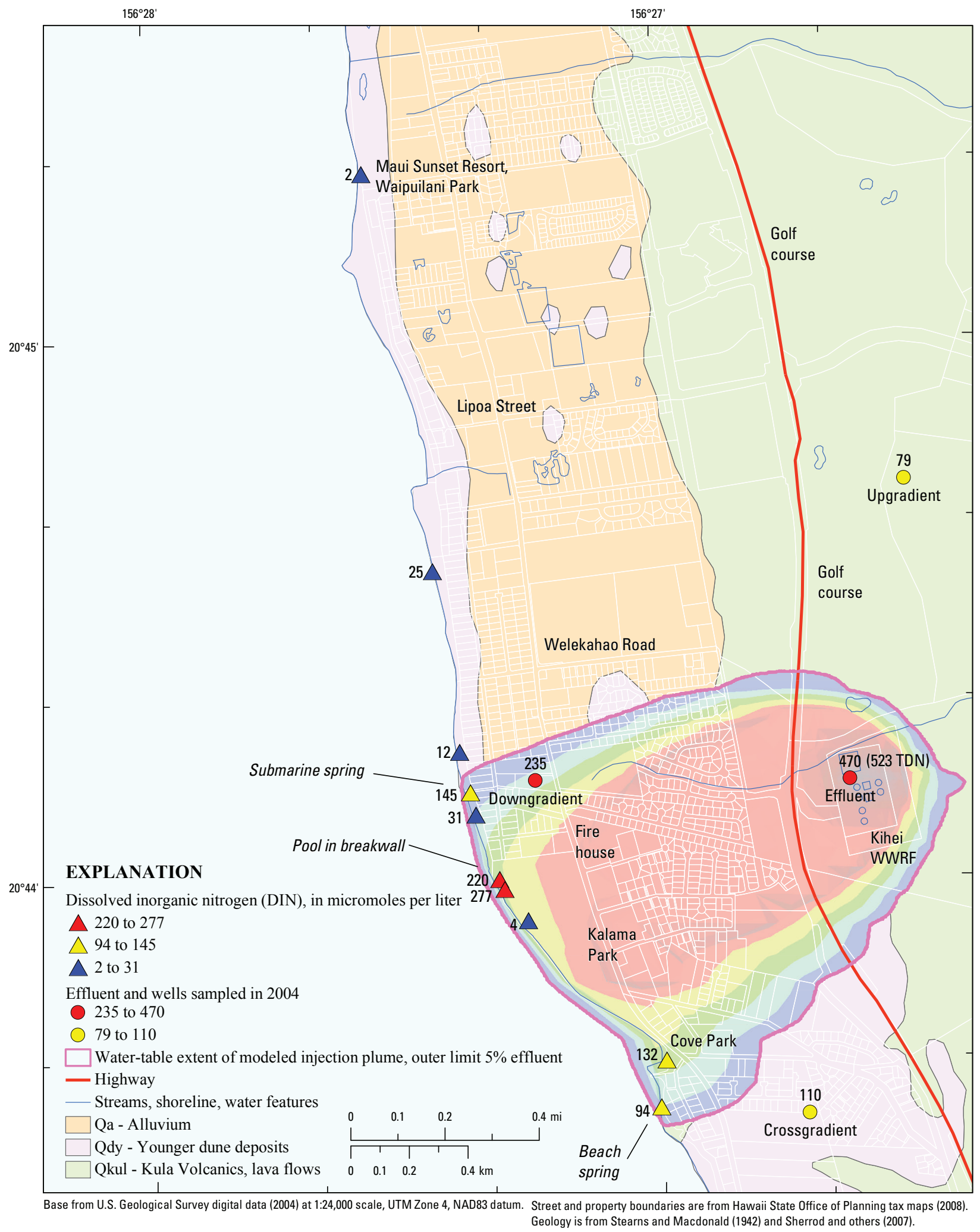

Figure 27. Map showing dissolved inorganic nitrogen (DIN) in water at Kihei, Hawaii. 
nitrogen pool in effluent than DIN. Because seawater is so low in nutrients $(\mathrm{DIN}=0.32 \mu \mathrm{M}$; see equation 1 , this report), measured DIN in our marine-water samples varied inversely with salinity, which explains why a low DIN concentration of $4 \mu \mathrm{M}$ could be measured in sample $\mathrm{K} 9$ right next to $277 \mu \mathrm{M}$ in sample K8, both in the heart of the modeled plume. Sample K9 was mostly seawater (only 5 percent fresh) and low in DIN, whereas K8 was only about half seawater (51 percent fresh) and its nonsaline component consists entirely of effluent at this central site.

Seawater at a reference site off Lahaina contained 0.32 $\mu \mathrm{M}$ DIN (Dollar and Andrews, 1997; see equation 1, this report), and Casciotti and others (2008) cited a mean nitrate concentration of $0.35 \mu \mathrm{M}$ at $150 \mathrm{~m}$ depth in open ocean water near Oahu. Compared to these concentrations, even upland water at Kihei is a high-nutrient source; for example, the 79.2 $\mu \mathrm{M}$ DIN in the upgradient well (table 3 ) is 248 times greater than in the Lahaina reference seawater. Effluent and fertilizers impart even larger contrasts: the $277 \mu \mathrm{M}$ DIN in marine sample K8 is 865 times greater than in the Lahaina reference seawater, although that factor surely will be reduced by further mixing on the reef flat. One implication of the strong differential in nutrient concentration between terrestrial waters and seawater is that it only takes a few percent terrestrial water to dominate the nutrient content of a mixed sample and produce a $\delta^{15} \mathrm{~N}$ composition that is essentially that of the terrestrial source. After unmixing the effect of seawater dilution, source DIN for sample K2 is $470 \mu \mathrm{M}$ (table 3), identical to the effluent DIN of $470 \mu \mathrm{M}$ and suggesting no nitrogen attenuation from injection to that sample. Sample K8 has an even larger source DIN of $541 \mu \mathrm{M}$ that exceeds effluent DIN and is closer to the effluent TDN of $523 \mu \mathrm{M}$. This could indicate that much of the additional organic nitrogen in effluent TDN is indeed part of the available nitrogen pool and is bacterially transformed to inorganic form once in the aquifer (by ammonification and later nitrification). Alternately, it could simply reflect time-varying DIN concentration in effluent and a higher DIN concentration when the K8 parent effluent was injected at some past time (about 1 to 3 years if the model estimates of groundwater age by Hunt, 2007, are accurate).

Ammonium (appendix B, fig. B15) may be a supporting wastewater tracer in this context, even if it is not solely diagnostic by itself. Samples with measurable ammonium lay within the modeled plume, and most had high nitrate and wastewater signatures of other tracers. These ammonium concentrations are higher than usually measured in Hawaiian groundwaters, at least in the volcanic-rock aquifers where water is generally oxic, low in dissolved carbon, and with nitrogen predominantly in the +5 valence (as nitrate) and ammonium typically not measurable (Hunt, 2004) or present at concentrations less than $0.2 \mu \mathrm{M}$ (Dollar and Atkinson, 1992). However, these generalizations probably do not hold true in coastal sediments and certainly are not true within high-nutrient point-source discharges like the Kihei effluent, which contained considerable ammonium $(19 \mu \mathrm{M})$, as did the downgradient well $(6.4 \mu \mathrm{M})$. Water from the downgradient well was anoxic or suboxic (Hunt, 2007), with reducing conditions at a nitrate-reduction redox level using the classification of McMahon and Chapelle (2008) and computer worksheet of Jurgens and others (2009). Reducing conditions would allow effluent ammonium to persist during transport in the aquifer and ultimately discharge at the shore. Notably, source ammonium concentrations in samples $\mathrm{K} 1, \mathrm{~K} 2$, and $\mathrm{K} 8$ were 11,24 , and $15 \mu \mathrm{M}$, respectively (table 3 ), comparable to the $19 \mu \mathrm{M}$ in effluent and possibly reflecting fairly direct transport and discharge of effluent ammonium from the injection plume. Ammonium could also be present in the marine samples as a result of nitrogen cycling in benthic sediments or water column. For example, Tribble and others (1990) reported ammonium as high as $76 \mu \mathrm{M}$ under reducing conditions in anaerobic reef porewater, and biota will cycle nitrogen back and forth among $\mathrm{N}$ species in benthic sediments and the water column. Reduction of nitrate to ammonium takes place mostly at night (Herzfeld and others, 2006), and there could have been some nighttime holdover on ammonium concentrations in our earlymorning samples. More sampling would be required to distinguish among these possibilities, but active plume discharge of ammonium is suggested at least for site $\mathrm{K} 1$ on the basis of the discharge rate of its submarine spring, which domes and roils the surface in waist-deep water.

Orthophosphate (appendix B, fig. B16) displayed a similar pattern as DIN and ammonium, with high $\mathrm{PO}_{4}$ concentrations in samples $\mathrm{K} 1, \mathrm{~K} 2$, and $\mathrm{K} 8$. However, unlike source DIN concentrations roughly comparable to effluent, source orthophosphate concentrations were much less than in effluent (table 3 ), with the maximum $15.3 \mu \mathrm{M}$ in sample $\mathrm{K} 8$ being only about one-fourth the $54.9 \mu \mathrm{M}$ in effluent. This indicates substantial loss of phosphorus during transport, most likely by sorption or other natural attenuation processes in the aquifer. Hunt (2007) measured about half as much nitrogen in the downgradient well as in effluent but only one-ninth the phosphorus concentration, and he attributed the concentration decrease mostly to actual removal mechanisms because dilution by lower-nutrient groundwater was only a second-order effect (the downgradient sample was at least three-quarters effluent according to $\delta^{2} \mathrm{H}$ and $\delta^{18} \mathrm{O}$ isotopes). The difference between the twofold and ninefold lowering of $\mathrm{N}$ and $\mathrm{P}$ was interpreted to mean that phosphorus attenuation in the aquifer was far more effective than nitrogen attenuation, proportionally.

Nitrogen-to-phosphorus ratios at Kihei (appendix B, fig. B17, and computed as DIN:PO ${ }_{4}$ ) appear to reflect this disproportional attenuation of $\mathrm{P}$ over $\mathrm{N}$, which is expected because of the greater solubility of nitrogen compared to phosphorus. Samples within the modeled plume certainly contain an effluent fraction (as confirmed by pharmaceuticals and heavy $\delta^{15} \mathrm{~N}$ ), but their N:P ratios ranging from 32 to 53 are much higher than the effluent ratio of 9, reflecting much greater proportional attenuation of $\mathrm{P}$ over $\mathrm{N}$ in the aquifer and nearshore marine zone. An exception is sample K9 in the heart of the plume, with an N:P ratio of 9, the same as in effluent. $\mathrm{N}: \mathrm{P}$ ratios have been computed and plotted here mainly for the 
benefit of marine ecologists who may wish to compare them against Redfield ratios that theoretically will govern phytoplankton uptake and other biotic assimilation of the groundwater-borne nutrients.

\section{Nutrients and Nitrogen Isotopes at Lahaina}

As at Kihei, nutrients and nitrogen isotopes also proved to be effective tracers for identifying injected wastewater effluent at Lahaina. Nitrogen isotopes in macroalgae (fig. 28 and table 7) progressed from light $\delta^{15} \mathrm{~N}$ compositions of +5 to +6 per mil along Honokowai Point to a maximum $\delta^{15} \mathrm{~N}$ of +39 per mil at Kahekili Beach Park, where the shallow reef shelf gave way to sand bottom from there south. Algae dry-weight nitrogen concentrations mimicked the $\delta^{15} \mathrm{~N}$ pattern (appendix $\mathrm{C}$, fig. C11), indicating that algae are bathed in higher nitrogen water in the southern half of the transect. The $\delta^{15} \mathrm{~N}$ of +1 per mil at the upland well can be interpreted as an end-member signature of agricultural fertilizers, a legacy effect of more than a century of sugarcane cultivation in the area (coffee is grown there currently). The slightly heavier $\delta^{15} \mathrm{~N}$ compositions of +5 to +6 per mil in algae along Honokowai Point may have evolved from slight denitrification from the upland $\delta^{15} \mathrm{~N}$ of +1 per mil, if upland water is the dominant source of groundwater discharge along the Point and if the well sample is representative of upland water generally.

There also was good agreement at Lahaina between $\delta^{15} \mathrm{~N}$ of algae and $\delta^{15} \mathrm{~N}$ of dissolved nitrate (fig. 29), for which water samples extended coverage south as far as Black Rock. Overall, Lahaina samples had much heavier $\delta^{15} \mathrm{~N}$ values than those at Kihei. Treated effluent (the starting point for further $\delta^{15} \mathrm{~N}$ evolution in the plumes) was +23 per mil at Lahaina and +15 per mil at Kihei. Algae $\delta^{15} \mathrm{~N}$ was as high as +39 per mil at Lahaina, twice the maximum of +18 per mil at Kihei. Also, the maximum $\delta^{15} \mathrm{~N}$ in water was +40 per mil at Lahaina (submarine spring samples L2 and L5), as compared to +23 per mil at Kihei (downgradient well). Intermediate $\delta^{15} \mathrm{~N}$ values of +13 per mil in sample L9 and lagoon sample L10 likely result from mixing of injected effluent and upland groundwater in the aquifer, although a possible confounding influence is golfcourse irrigation with reclaimed water in that area. Viewed jointly, the high algae nitrogen concentrations and heavy $\delta^{15} \mathrm{~N}$ values constitute conclusive evidence that nitrogen-rich effluent discharges along the beach southwest of the Lahaina injection wells, and that denitrification is active in the Lahaina effluent plume, as it is at Kihei.

Dissolved nitrogen in Lahaina effluent was $366 \mu \mathrm{M}$ as DIN and $437 \mu \mathrm{M}$ as TDN (fig. 30 and table 4), both of which were lower than at Kihei ( $470 \mu \mathrm{M}$ DIN and $523 \mu \mathrm{M}$ TDN). Submarine spring samples L1, L2, and L5 had DIN concentrations ranging from 52 to $62 \mu \mathrm{M}$, and they certainly contain an effluent fraction as confirmed by pharmaceuticals, waste indicator compounds, and heavy $\delta^{15} \mathrm{~N}$. Sample L2 had a source DIN of $333 \mu \mathrm{M}$ (table 4), nearly as much as the $365 \mu \mathrm{M}$ in effluent and at first glance suggesting little attenuation of DIN from point of injection to that sample location. However, the heavy $\delta^{15} \mathrm{~N}$ of +40 per mil in sample L2 indicates considerable denitrification from the effluent $\delta^{15} \mathrm{~N}$ of +23 per mil. The combination of heavy $\delta^{15} \mathrm{~N}$ and high DIN in sample L2 may be another sign that effluent TDN is a more relevant measure of the total nitrogen pool available for nitrogen cycling than is effluent DIN. Samples L1 and L5 had source DIN about 100 $\mu \mathrm{M}$ less than L2 but roughly equal nitrate + nitrite, the main difference being the large ammonium fraction $(105 \mu \mathrm{M})$ in L2. All three samples had very similar $\delta^{15} \mathrm{~N}$ values, suggesting a similar degree of denitrification for all. Notably, sample L5 is from the same spring as L2, only they were sampled a few hours apart. These differences at the same site indicate that conditions are more complex than can be explained in simple terms and illustrate the limits of using these point-intime samples to infer plume processes. Geochemical gradients within the plume probably are steep and impart large sampling variability at short time scales, such as from wave and tidal forcing. Attempts at relating sampled effluent to environmental samples taken at distances far from the injection point are also subject to discontinuities at longer time scales, including day-to-day variations in effluent composition at the treatment plant and the probable weeks-to-months traveltime from injection to sample location. However, short of monitoring around the clock as was done by Herzfeld and others (2006), it is still possible to glean some interpretive value from reconnaissance sampling such as this.

Upland well sample L11 at Lahaina had $254 \mu \mathrm{M}$ DIN, more than twice the $79-110 \mu \mathrm{M}$ DIN in upland wells at Kihei and reflecting a large fertilizer nitrogen contribution at Lahaina from former plantation agriculture. The $236 \mu \mathrm{M}$ DIN in lagoon sample L10 at Black Rock is exactly commensurate with slight seawater dilution of upland groundwater, because the lagoon sample was 93 percent fresh and L10 source DIN equals the L11 upland water DIN of $254 \mu \mathrm{M}$ (table 4). The upland well sample and historical data allow a few judgments regarding fertilizer-derived nutrients. Soicher and Peterson (1997) cited an upper bound of $0.3 \mathrm{mg} / \mathrm{L}(21 \mu \mathrm{M})$ nitrate for wells upgradient of agricultural fields in the Lahaina area, a forest "background" concentration that is 65 times greater than the $0.32 \mu \mathrm{M}$ seawater DIN locally (Dollar and Andrews, 1997; see equation 1, this report). The upland well DIN of $254 \mu \mathrm{M}$ is 12 times greater than forest background and nearly three orders of magnitude greater than the seawater concentration. The upland well DIN also is only 31 percent less than Lahaina effluent DIN and 42 percent less than effluent TDN. Also shown in fig. 30 is a nitrate+nitrite concentration of $314 \mu \mathrm{M}$ at well 5641-01 obtained in February 1980 (Souza, 1981), more than two years before the injection wells were placed in operation in May 1982 (County of Maui, 1983) and when agricultural fertilizers would have been the only large nutrient source in the vicinity. Other high nitrate concentrations reported for cultivated areas include $364 \mu \mathrm{M}$ at Olowalu, West Maui (Souza, 1981) and 515 $\mu \mathrm{M}$ in central Maui (Takasaki, 1972). Several conclusions can be drawn from these data: (1) fertilizer-affected groundwater on Maui can be comparable in inorganic nitrogen concentration to treated wastewater; (2) nitrogen concentrations in 


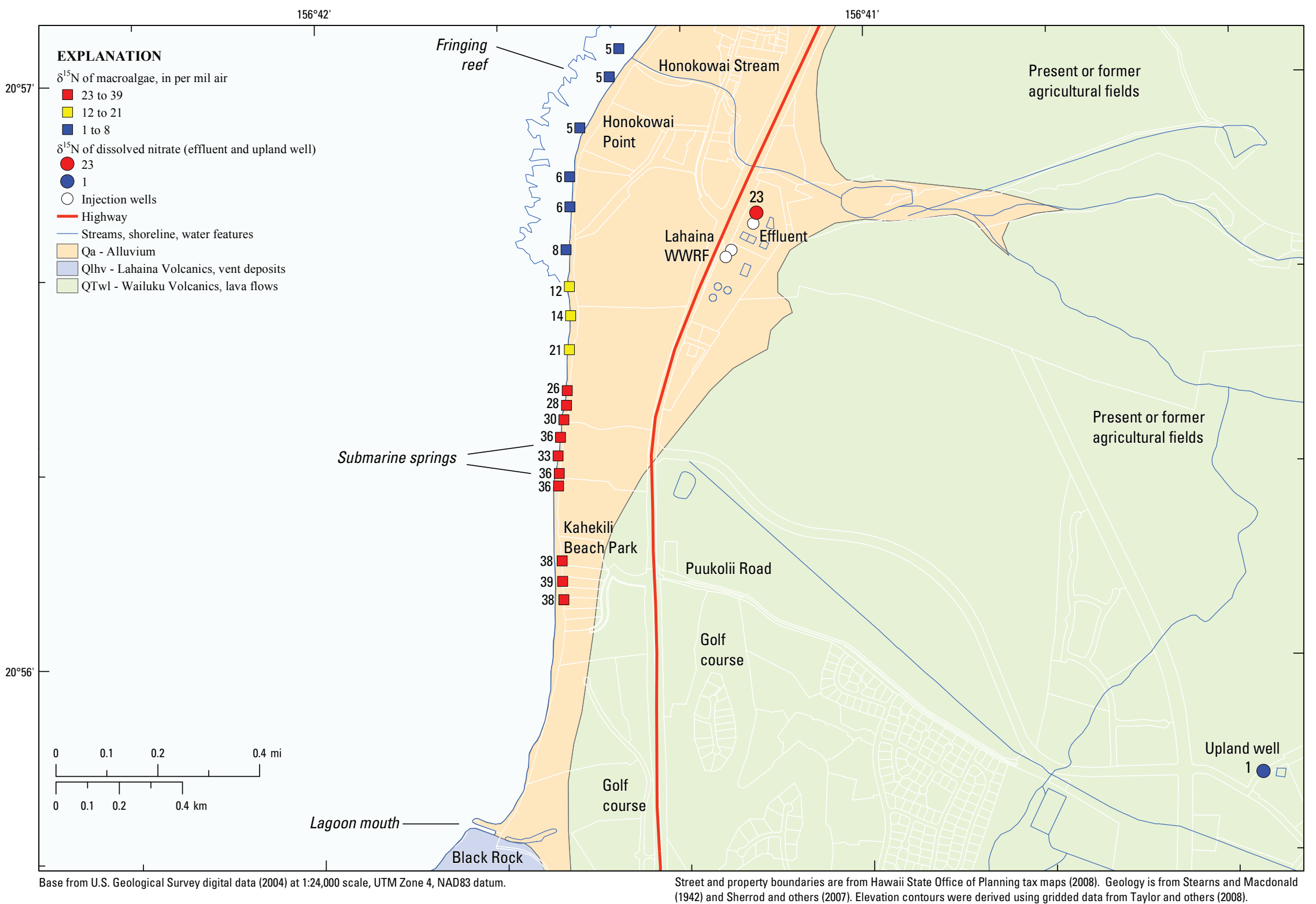

Figure 28. Map showing $\delta^{15} \mathrm{~N}$ isotopic composition of macroalgae at Lahaina, Hawaii. 


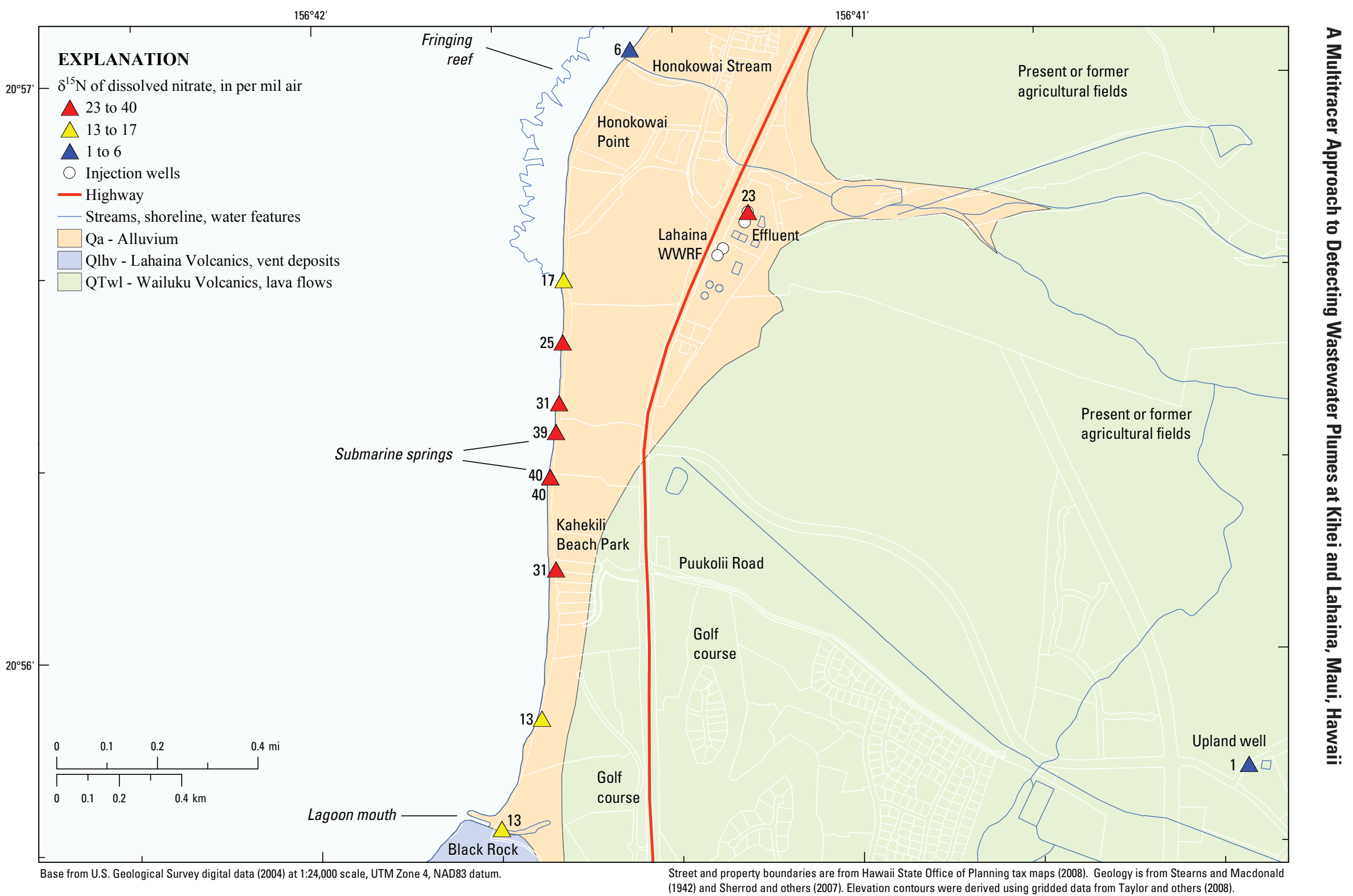

Figure 29. Map showing $\delta^{15} \mathrm{~N}$ isotopic composition of dissolved nitrate at Lahaina, Hawaii. 


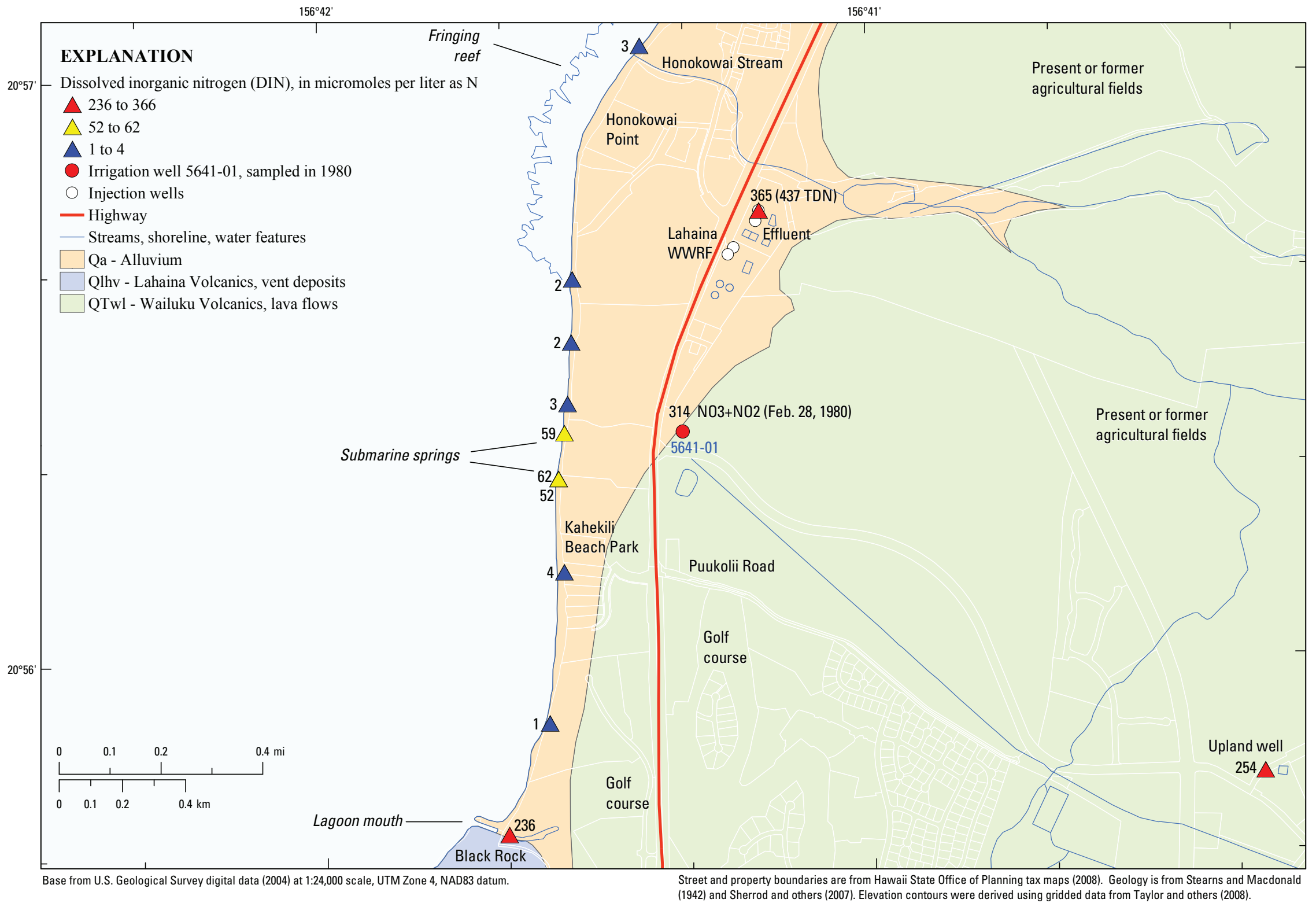

Figure 30. Map showing dissolved inorganic nitrogen (DIN) in water at Lahaina, Hawaii. 
fertilizer-affected groundwater exceed forest background by an order of magnitude and exceed reference seawater concentrations by three orders of magnitude; and (3) fertilizer-derived nitrogen still persists at high concentrations at Lahaina even though intensive sugarcane cultivation ceased a decade ago in 1999. This is consistent with hydrologic understanding of groundwater flushing times, which are on the order of decades, even without considering storage in soil and the unsaturated zone. For example, Liu (2007) computed a "mean hydraulic residence time for water particles in the Iao aquifer" of West Maui to be 41.5 years. The computation was for the fresh groundwater lens, excluding the unsaturated zone.

Ammonium was detected at the submarine springs and upland well (appendix C, fig. C15) and was an order of magnitude higher in effluent than at Kihei. Sample L2 had a source ammonium of $105 \mu \mathrm{M}$, about 44 percent less than the $189 \mu \mathrm{M}$ in effluent (table 4). Some of that ammonium loss may have been to volatilization or annamox, but some may also have been nitrified to nitrate if sufficient oxygen was present at some points along the water's flowpath. There seems to be some support for this in the data. Samples L1, L2, and L5 have source nitrate+nitrite concentrations that exceed that in effluent despite also having heavy $\delta^{15} \mathrm{~N}$ compositions indicating nitrogen loss by denitrification. This contradiction is explainable if much of the effluent ammonium and organic nitrogen (fig. 22) are available to the total nitrogen pool in the aquifer through transformations. Some ammonium appears to persist in the injection plume under anoxic conditions like those in the Kihei plume, if sample L2 reflects ammonium transport and not simply a transient reduction to ammonium in the benthic subsurface. The spring samples were collected by inverted-funnel sampling at the sea bottom, and this argues for active discharge of ammonium from the effluent plume. Coral cobbles on the bottom had a black mineral coating on their underside, most likely manganese oxide or iron sulfide that would indicate reducing conditions. Similar black coatings were described at the Cape Cod sewage plume and were determined to be manganese oxyhydroxide (Walter and others, 1996). The Lahaina mineral coatings suggest reducing conditions sufficient to mobilize manganese or iron into solution in groundwater, followed by a change from reducing to oxidizing conditions when groundwater contacts oxic seawater as it discharges, causing mineral precipitation. A manganese oxide coating would indicate manganese reduction, the next redox step after nitrate reduction. An iron sulfide coating would indicate iron and sulfate reduction, farther along the redox sequence just short of methanogenesis (McMahon and Chapelle, 2008). Whatever the mineral coating may be, it indicates that conditions in the plume are sufficiently reducing to maintain and transport ammonium from point of injection to the submarine springs. Conditions that reducing would also tend to imply a low-nitrate or nitrate-free zone, where much or all nitrate has been denitrified and only ammonium is present, as at Cape Cod (LeBlanc, 1984; Bohlke and others, 2006). Because our samples did not show this, it could mean that there are zones in which plume geochemistry is substantially different from what was seen in this study. It could also simply mean that it is not possible to observe such chemistry with water-column sampling - for example, if there are last-minute chemical transformations in the shallow benthic layer just before groundwater discharges from the ocean bottom or submarine springs. Ammonium transport in the Cape Cod sand and gravel is retarded by cation exchange, resulting in an ammonium cloud that moves at slower velocity than groundwater flow and conservative constituents, and that has not yet reached its discharge boundary at a nearby lake (Bohlke and others, 2006). That appears not to be the case here, where there is evidence for ammonium transport and discharge at the shore from both the Kihei and Lahaina plumes.

Orthophosphate was high in concentration at the submarine springs and lagoon at Black Rock (appendix C, fig. C16), though it was highest in the upland well at $7.7 \mu \mathrm{M}$, surprisingly higher than in effluent. However, the effluent concentration of $5 \mu \mathrm{M}$ was an order of magnitude less than at Kihei, and spring samples L1 and L5 had nearly as much $\mathrm{PO}_{4}(4.0$ and $4.2 \mu \mathrm{M}$, respectively) as effluent, despite being diluted by seawater to only $24-26$ percent fresh. Source orthophosphate concentrations of $16 \mu \mathrm{M}$ are estimated for these samples (table 4), or more than 3 times the measured concentration in effluent. Possibilities are that effluent was abnormally low in phosphorus on the day sampled, such as from day-to-day variation in removal efficiency during treatment, or that the elevated source concentrations at the springs could reflect desorption of excess phosphorus stored in the aquifer (sorbed to aluminumand iron-oxide mineral coatings; Walter and others, 1996), perhaps as a transient response to some changing condition. There is no way to know from this dataset alone, given transient forcing on tidal and other time scales, samples taken at a single point in time, the highly nonconservative behavior of phosphorus, and a variety of other uncertainties. A clearer situation exists at the south lagoon: source orthophosphate concentration in sample L10 $(2.1 \mu \mathrm{M}$; table 4$)$ is lower than both effluent $(5.0 \mu \mathrm{M})$ and upland well water $(7.7 \mu \mathrm{M}$, sample L11), possibly as a result of phosphorus sorption within the aquifer or lagoon-bottom muds but more likely as a result of $\mathrm{PO}_{4}$ utilization in the bright green phytoplankton bloom within the lagoon. The orthophosphate concentration of $7.7 \mu \mathrm{M}$ in the upland well at Lahaina (table 4) was considerably higher than the 1.6 and $1.9 \mu \mathrm{M}$ in the upgradient and crossgradient wells at Kihei (table 3), again reflecting the additional nutrient contribution from agricultural fertilizers at Lahaina.

Nitrogen-to-phosphorus ratios at Lahaina (appendix C, fig. C17) showed several interesting patterns. The N:P ratio of 74 in Lahaina effluent was much larger than the ratio of 9 in Kihei effluent (appendix B, fig. B17), mainly as a result of the order-of-magnitude lower $\mathrm{PO}_{4}$ concentration at Lahaina (table 3 and table 4). Ratios of 15-21 at submarine spring samples L1, L2, and L5 are greatly reduced from the 74 ratio in the Lahaina effluent, and - if representative - they reflect disproportional attenuation of $\mathrm{N}$ over $\mathrm{P}$ in the Lahaina plume, the opposite sense from the P-over-N attenuation at Kihei. The N:P ratio of 120 in lagoon sample L10 was the highest 
of all samples, some 3.6 times the upland ratio of 33, mainly because $\mathrm{PO}_{4}$ concentration in the lagoon sample is lower by a similar factor while DIN is only slightly lower (table 4).

\section{Hydrogen and Oxygen Stable Isotopes of Water}

Stable-isotope compositions $\delta^{2} \mathrm{H}$ and $\delta^{18} \mathrm{O}$ characterize the hydrogen and oxygen atoms in the water molecule, $\mathrm{H}_{2} \mathrm{O}$. Fractionation of water is a partitioning process whereby isotopic composition is changed mainly during phase changes: during condensation from water vapor or evaporation from liquid water back to vapor. These processes occur in clouds where water precipitates, on the landscape where some fraction of precipitation evaporates, and by evaporation from standing water (lakes, streams, marshes, and ocean). A useful property of water isotopes is that once water infiltrates to the water table, $\delta^{2} \mathrm{H}$ and $\delta^{18} \mathrm{O}$ tend to remain fixed and behave conservatively (not changing, other than by simple mixing) during groundwater transport to destinations farther along the flow system. This makes them good tracers for some waters, although mixing calculations may be needed to make sense of the data. Groundwaters recharged at different elevations in Hawaii obtain distinctive compositions (Scholl and others, $1995,2002)$ that contrast strongly against seawater.

At Kihei, $\delta^{2} \mathrm{H}$ and $\delta^{18} \mathrm{O}$ discriminate effluent from upland groundwater quite well (appendix B, fig. B18, B19). Effluent originates from tap water that is piped in from the north side of the island and that is isotopically heavier than local Kihei groundwater. The heavy signature persists through waste treatment and is retained in the effluent. The large difference of 28 per mil between effluent $\delta^{2} \mathrm{H}(-15.1$ per mil, Table 3$)$ and $\delta^{2} \mathrm{H}$ in the upgradient well ( -43.2 per mil) helps discriminate the two waters. Groundwater from the downgradient well near the plume periphery has an intermediate $\delta^{2} \mathrm{H}$ value of -21.6 per mil that can be interpreted mainly as a mixture of these two terrestrial sources (effluent and upland groundwater). Using the isotopic values in a proportional mixing calculation, one can estimate that the downgradient well contains about 75 percent effluent and 25 percent upland groundwater, and its salinity indicates it contains about 1 percent more seawater than water from the upgradient well.

This type of simple binary discrimination fails along the shore, however, where seawater becomes a major third component, with seawater dilution driving $\delta^{2} \mathrm{H}$ and $\delta^{18} \mathrm{O}$ values toward heavier compositions near zero. Nearshore marinewater samples can be a mixture of all three waters (effluent, upland groundwater, seawater), and without multicomponent mixing calculations one cannot judge whether seawater is blending with effluent, upland water, or an effluent-upland mixture. Beach spring sample K10 is an exception: it is 95 percent fresh and it clearly inherits its $\delta^{2} \mathrm{H}$ value of -43.5 per mil from upland water like that at the nearby crossgradient well $(-44$ per mil). This constrains the southern limit of the effluent plume, in that K10 appears to consist almost entirely of upland water on the basis of its $\delta^{2} \mathrm{H}$ value. The same interpretations could be made using $\delta^{18} \mathrm{O}$ (appendix B, fig. B19), the main difference being a scale factor in the numeric values.

At Lahaina (appendix C, fig. C18, and table 4), $\delta^{2} \mathrm{H}$ only differed by 3.1 per mil between effluent $(-10.9$ per mil) and the upland well ( -14 per mil). The only sample fresh enough to be constrained by those extremes is lagoon sample L10, whose $\delta^{2} \mathrm{H}$ composition of -12.3 per mil is intermediate and somewhat closer to upland water than effluent. All other values are dominated by seawater, and even at the submarine springs (15-26 percent fresh) it is not possible to infer whether the fresh fraction originates more from effluent or upland groundwater on the basis of $\delta^{2} \mathrm{H}$ or $\delta^{18} \mathrm{O}$.

\section{Mixing and Chemical Evolution of Injected Effluent}

Beyond interpreting the data with mapping approaches, certain aspects of effluent evolution and mixing with adjacent groundwaters can be revealed by plotting nutrients and stable isotopes measured in this study on diagrams. Simple mixtures of source waters will often plot along straight lines on such diagrams, which are sometimes referred to as mixing plots. Most of the water samples collected in the field are mixtures of two or more source waters, and mixing plots and calculations can help in comparing samples and interpreting their significance, for example to bound the lateral limits of the injection plumes or to document natural attenuation of nutrients apparently taking place within the plumes.

Known source waters that were sampled or for which compositions can be taken from the literature include wastewater effluent, upland groundwater, and seawater. Additional sources not sampled, but which may be present in some quantity, include groundwater recharged in the lowlands (between sampled wells and the shore, and possibly gaining more nutrients from urban infiltration) and cesspools or leaking sewer lines (which also would contribute additional nutrients if present).

\section{Basis for Mixing Plots and Calculations}

The following discussion makes use of binary (two-component) mixing plots and calculations. Multicomponent calculations might be useful (for example effluent-groundwaterseawater) but are more complex, and favorable circumstances combine so that a binary approach is adequate for many interpretations. The first circumstance is the much greater nutrient content in terrestrial waters than in seawater in the study area, which contains almost no nutrients by comparison. Effluent and groundwater both impart a high-nutrient signal that stands out against a low-nutrient seawater background, and nearly all nutrients can be considered land-derived for our purposes. Secondly, there is good $\delta^{15} \mathrm{~N}$ contrast between effluent and upland groundwater; they can be discriminated readily even 
in marine-water samples, where only a few percent terrestrial water can contribute the bulk of the nitrogen and dominate the $\delta^{15} \mathrm{~N}$ signature. Finally, seawater can be removed as a third component by an unmixing calculation that estimates nutrient source concentrations in the fresh fraction of sampled waters (equation 1 in this report).

Mixing plots were constructed by drawing straight lines between source waters to represent conservative mixing, and lines were computed where necessary for isotopic mixing and fractionation, which do not produce simple straight lines. Proportional mixing of dissolved constituents (nutrients, salinity) was computed as:

$$
C_{m i x}=f_{1} C_{1}+\left(1-f_{1}\right) C_{2}
$$

where:

$C_{m i x}$ is the concentration in the mixture;

$C_{1}$ is the concentration in component 1 ;

$C_{2}$ is the concentration in component 2 ;

$f_{1}$ is the fraction of component 1 ;

$\left(1-f_{1}\right)$ is the fraction of component 2 ; and where:

$f_{1}+\left(1-f_{1}\right)=1$ (simply, the two fractions make up the whole).

For stable isotopes of dissolved constituents, there is the additional complication that isotopic compositions must be weighted by the concentration in the sample (the mass of element dissolved in it). For example, a two-component mixing model for $\delta^{15} \mathrm{~N}$ in dissolved nitrate is:

$$
\begin{gathered}
\delta^{15} \mathrm{~N}_{\operatorname{mix}}=\left(f_{1} C_{1} \delta^{15} \mathrm{~N}_{1}+\right. \\
\left.\left(1-f_{1}\right) C_{2} \delta^{15} \mathrm{~N}_{2}\right) /\left(f_{1} C_{1}+\left(1-f_{1}\right) C_{2}\right)
\end{gathered}
$$

where:

$\delta^{15} \mathrm{~N}_{\text {mix }}$ is the nitrogen-isotope composition of the mixture;

$\delta^{15} \mathrm{~N}_{1}$ is the nitrogen-isotope composition of component 1 ;

$\delta^{15} \mathrm{~N}_{2}$ is the nitrogen-isotope composition of component 2 , and

remaining terms are as defined for equation 3 above.

Denitrification lines were also computed and displayed on the mixing plots to show evolution of $\delta^{15} \mathrm{~N}$ compositions and residual DIN concentrations. Denitrification lines were computed using the Rayleigh fractionation equation (chapter 16 in Kendall and McDonnell, 1998):

$$
\delta^{15} \mathrm{~N}_{\text {residual }}=\delta^{15} \mathrm{~N}_{\text {initial }}+\varepsilon(\ln f)
$$

where:

$\delta^{15} \mathrm{~N}_{\text {initial }}$ is the starting nitrogen-isotope composition of the nitrate pool;

$\delta^{15} \mathrm{~N}_{\text {residual }}$ is the nitrogen-isotope composition in the residual or remaining nitrate pool;

$\varepsilon$ is the enrichment factor, a rate constant associated with the fractionation process;

In is the natural logarithm; and $f$ is the fraction of the original nitrate pool remaining, also expressed as $C / C_{0}$ (residual concentration divided by initial concentration).

The enrichment factor, $\varepsilon$, was determined by trialand-error adjustment until a line was fit connecting effluent composition with a denitrified target composition such as the downgradient well at Kihei or submarine spring sample L5 at Lahaina.

All equations were calculated in a computer spreadsheet, with values calculated at 1-percent mixture intervals in mixing equations and at 1-percent intervals of $\mathrm{f}$ (the fraction of nitrate remaining) in the fractionation equation. DIN was used as the nitrogen species in plots and calculations to account for all relevant inorganic nitrogen (including ammonium, which can be transformed to nitrate readily if sufficient oxygen is present). DIN was used even in fractionation calculations, despite the denitrification process pertaining strictly only to nitrate.

As a result, fitted values of the enrichment factor, $\varepsilon$, may not be entirely accurate. However, denitrification calculations at Lahaina wouldn't be possible if restricted only to nitrate, because source nitrate concentrations in destination samples were higher than in effluent (table 4), not lower. The premise employed here is that much of the ammonium in effluent may be available for nitrification to nitrate at some point and should be included as DIN in the calculations. We don't contend that this is true on theoretical grounds, we simply employ it here as an artifice; otherwise, one cannot make sense of observed changes in $\delta^{15} \mathrm{~N}$ and nitrogen concentration jointly, particularly for the Lahaina effluent that contains a large proportion of ammonium. We are reducing a complex situation in which several processes are at work to one single "denitrification trend" to compare against our data and evaluate the plausibility of the results. Other processes, such as sorption or nitrification of ammonium, may also be affecting the $\delta^{15} \mathrm{~N}$ composition of nitrate in our samples, but we certainly cannot sort those effects out in this reconnaissance-level study.

Another simplification that must be emphasized is that we are comparing samples that do not match up as "contemporaneous" in any genetic sense. That is, although we compare marine-water samples collected in 2008 to effluent sampled in 2008 (Lahaina) and effluent sampled in 2004 (Kihei), neither comparison is strictly correct because for all samples containing an effluent fraction, that source effluent was injected at some prior time that is unknowable and that differs from sample to sample and from the effluent samples used for comparison. For purposes of discussion, we will assume relatively steady effluent compositions and plume conditions over time. Some temporal variation in effluent nutrient concentrations and isotopic compositions is expectable; however, the basic premise for this discussion is that variation in the data mainly reflects spatial plume zonation, because our samples transect across a broad range in geochemical processes and dilution gradients from plume core to outer periphery, blending to ambient groundwater laterally and to saltwater toward the shore. Regarding effluent traveltime, Hunt (2007) estimated 
by modeling the Kihei plume that "discharge from most of the plume is 5 years or younger and is as young as 1 year at the plume apex." Lesser traveltime, on the order of months to several years, might be expected for Lahaina, where injection wells are closer to shore than at Kihei.

\section{Mixing and Effluent Evolution at Kihei}

Stable isotopes of water appear to follow conservative mixing at Kihei and can be used to evaluate possible mixtures in the various samples before considering the nonconservative behavior of nutrients. A "seawater mixing plot" can be made for $\delta^{2} \mathrm{H}$ versus salinity (fig. 31 ), showing three principal endmember components (effluent, upland groundwater, seawater) and with straight lines drawn to represent conservative, binary mixing between any two components. A local seawater composition has been projected from the most saline sample K9 (95 percent seawater), assuming its fresh fraction is effluent. Most samples plot above a line connecting upland water with the zero isotopic composition of VSMOW (Vienna Standard Mean
Ocean Water), suggesting that local seawater is slightly heavier isotopically than SMOW. The local seawater composition is $\delta^{2} \mathrm{H}=+3$ per mil and $\delta^{18} \mathrm{O}=+0.4$ per mil, with $\delta^{18} \mathrm{O}$ estimated from a similar plot (not shown).

The various samples plot relative to the end members in sensible ways, although inference is limited by the close convergence of mixing lines at high salinity. At the left side of the plot, the large isotopic separation between effluent and upland groundwater is clear. The downgradient well has an intermediate composition between effluent and upland water but plots closer to effluent. Hunt (2007) estimated the downgradient well water to be roughly three-quarters effluent on the basis of these isotopes. The downgradient well contains a small amount of seawater ( 5 percent), pulling it slightly to the right of the upland-effluent mixing line. Samples K4 and K7 plot close to the upland-seawater mixing line, consistent with their areal locations well outside the modeled injection plume (fig. 9). Samples K2 and K8 plot close to the effluent-seawater mixing line, consistent with their locations in the core of the plume near K9. Samples K1, K3, K5, and K6 have intermediate

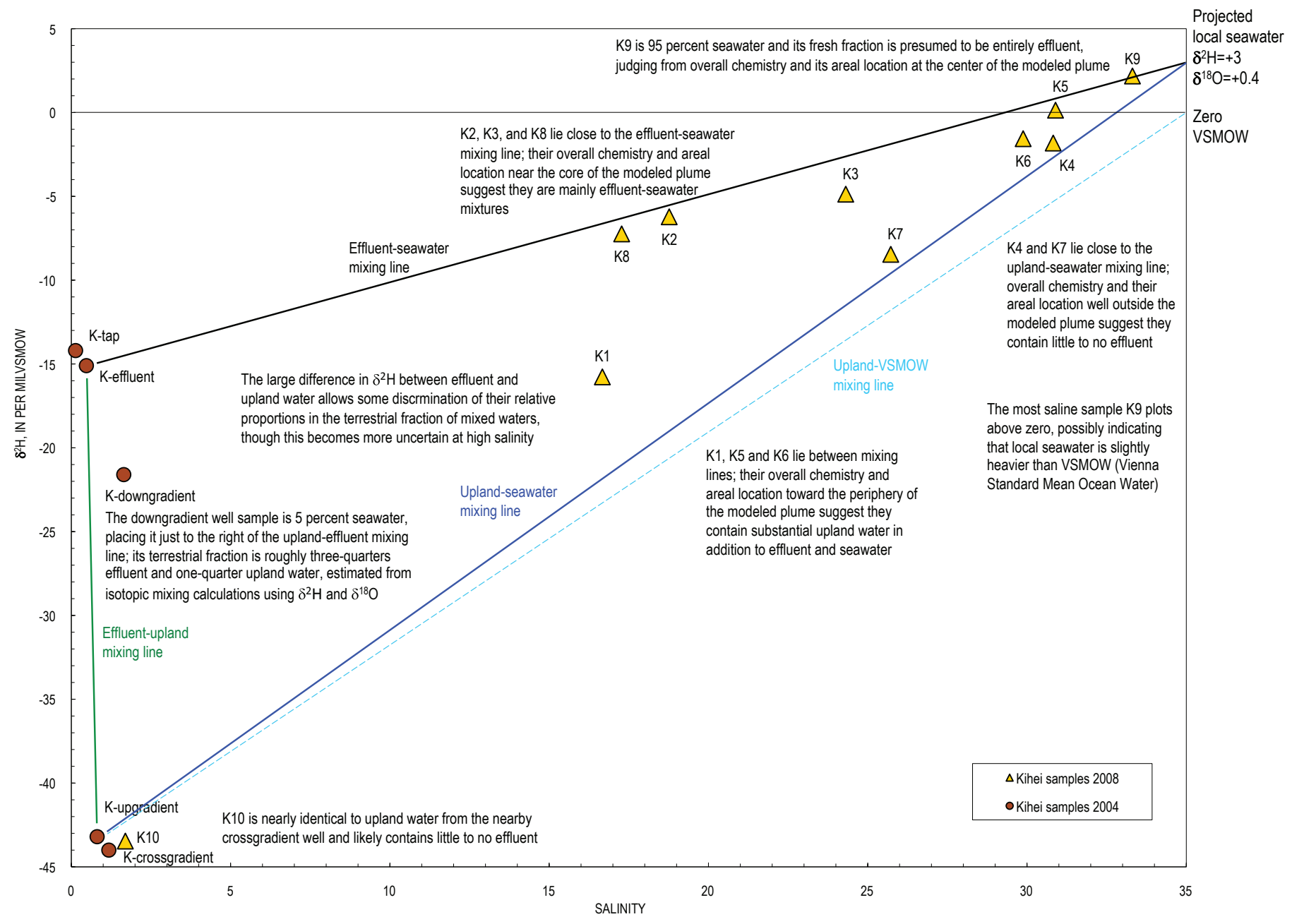

Figure 31. Seawater mixing plot showing $\delta^{2} \mathrm{H}$ versus salinity at Kihei, Hawaii. 
compositions between the mixing lines that are consistent with their locations more to the periphery of the injection plume, where they are expected to contain some upland water mixed in with effluent. Sample K10 plots right next to the nearby crossgradient well, and one can infer that K10 contains very little or no effluent.

Seawater mixing plots for nutrients (fig. 32) show a range of behavior between conservative and nonconservative mixing. For dissolved inorganic nitrogen (fig. 32A), a few samples plot on or close to the effluent-seawater mixing line (K2, K3, $\mathrm{K} 8$ ), indicating that they consist mainly of effluent diluted by seawater. Samples thought to originate entirely from upland water (K4, K5, K7, K10) plot on or near the upland-seawater mixing line, and probable mixtures of effluent and upland water (K1, K6, downgradient well) plot between mixing lines for the most part. However, there are some discrepancies indicating nonconservative loss of nitrogen. Sample K9 plots well below effluent-seawater mixing even though its areal location in the heart of the plume suggests it should be an exclusive mixture of the two. The downgradient well plots less than halfway between upland water and effluent, though it plotted three-quarters of the way toward effluent on the $\delta^{2} \mathrm{H}$ mixing plot. Both of these samples suggest a loss of DIN over and above simple seawater mixing. Orthophosphate loss is even more pronounced (fig. 32B): all samples plot well below the effluent-seawater mixing line, even those whose fresh fraction is presumed from other evidence to be mostly or entirely effluent (K1, K2, K3, K8, downgradient well). Viewed jointly, the DIN and orthophosphate mixing plots reflect net loss of both nutrients from an effluent starting composition and proportionally greater loss of $\mathrm{P}$ over $\mathrm{N}$, as proposed previously by Hunt (2007).

Considering nitrogen more closely, figure 33 reproduces the same seawater mixing plot for DIN in the upper panel A,
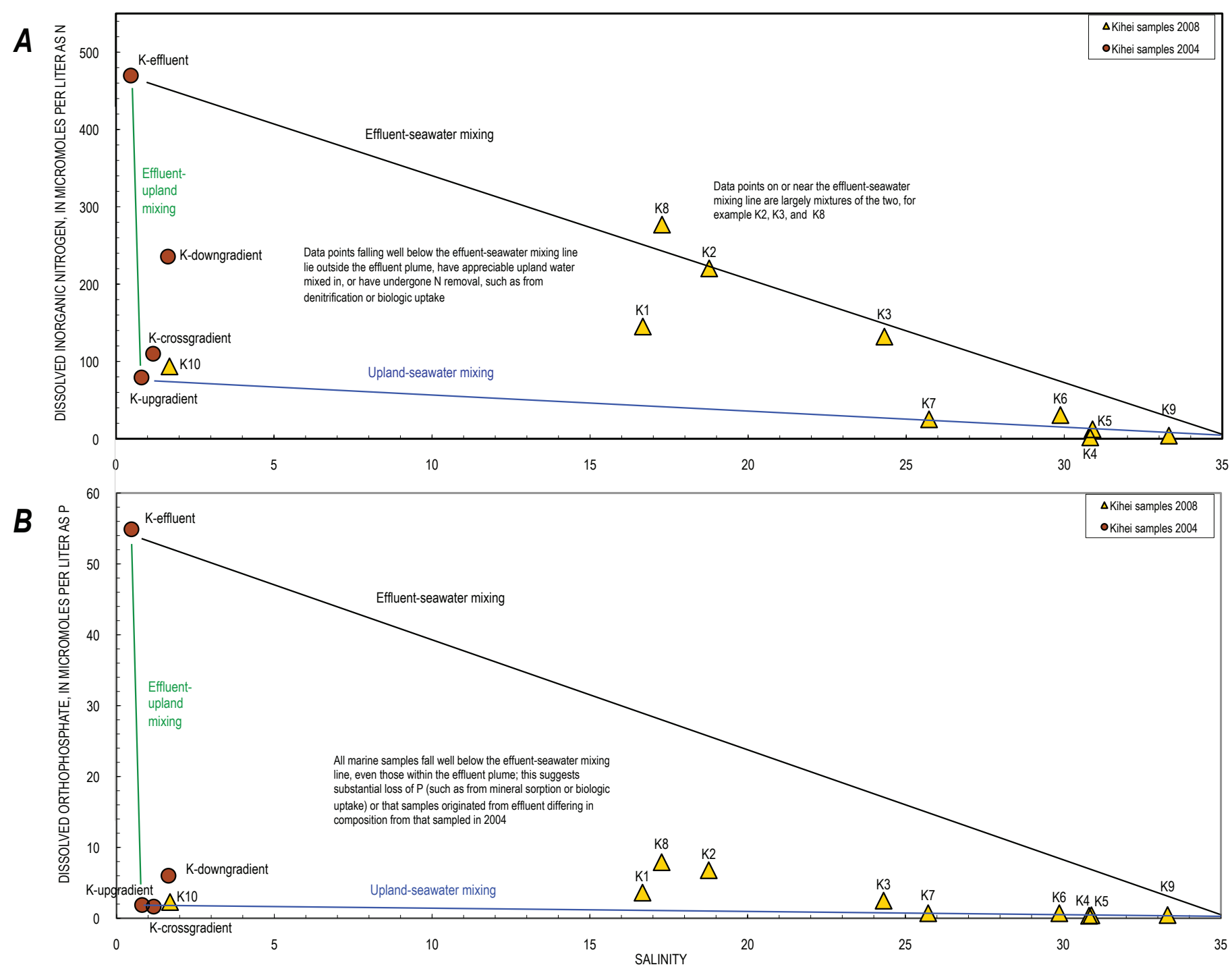

Figure 32. Seawater mixing plots for $(A)$ dissolved inorganic nitrogen, and $(B)$ orthophosphate in water samples at Kihei, Hawaii. 
while the bottom panel B substitutes source DIN concentrations resulting from seawater unmixing calculations (table 3 ). Mixing lines are not drawn in the bottom panel (seawater dilution has been "unmixed" by the calculation), and the main exercise is to compare source DIN concentrations to possible parent concentrations, which are marked by horizontal reference lines. That is, one is most interested in whether data points lie above or below the parent concentrations, and by how much. Source DIN concentrations in samples K2 and $\mathrm{K} 3$ nearly equal effluent DIN, suggesting little modification or net loss of effluent nitrogen in these samples. Sample K8 has a source DIN concentration that far exceeds effluent DIN and plots nearer to effluent TDN. This could indicate that the organic nitrogen component of TDN has been converted to inorganic form, that effluent DIN varied over time and was higher when $\mathrm{K} 8$ effluent was injected in the past, or that additional nitrogen may have been contributed as groundwater flowed under the urban strip from point of injection to the shore. Samples lying in or near the injection plume but with source DIN much less than effluent (K1, K5, K6, K9, downgradient well) may reflect dilution by lower nitrogen upland groundwater, loss of nitrogen by denitrification, or both (although K9 probably contains no upland water, given its central location in the injection plume). Samples K7 and K10 have source DIN concentrations essentially identical to their expected parent upland water. However, sample K4 has far less source DIN than upland water, and this suggests substantial nitrogen loss by biologic uptake, because the sample does not have a heavy $\delta^{15} \mathrm{~N}$ composition that would indicate $\mathrm{N}$ loss by denitrification (fig. 26).

Stable isotopes of nitrate reveal biochemical evolution of injected effluent in the aquifer and nearshore marine zone. Figure $34 A$ shows $\delta^{18} \mathrm{O}$ versus $\delta^{15} \mathrm{~N}$ of dissolved nitrate, the same plot as the nitrate-source classification of figure 24. Hypothetical
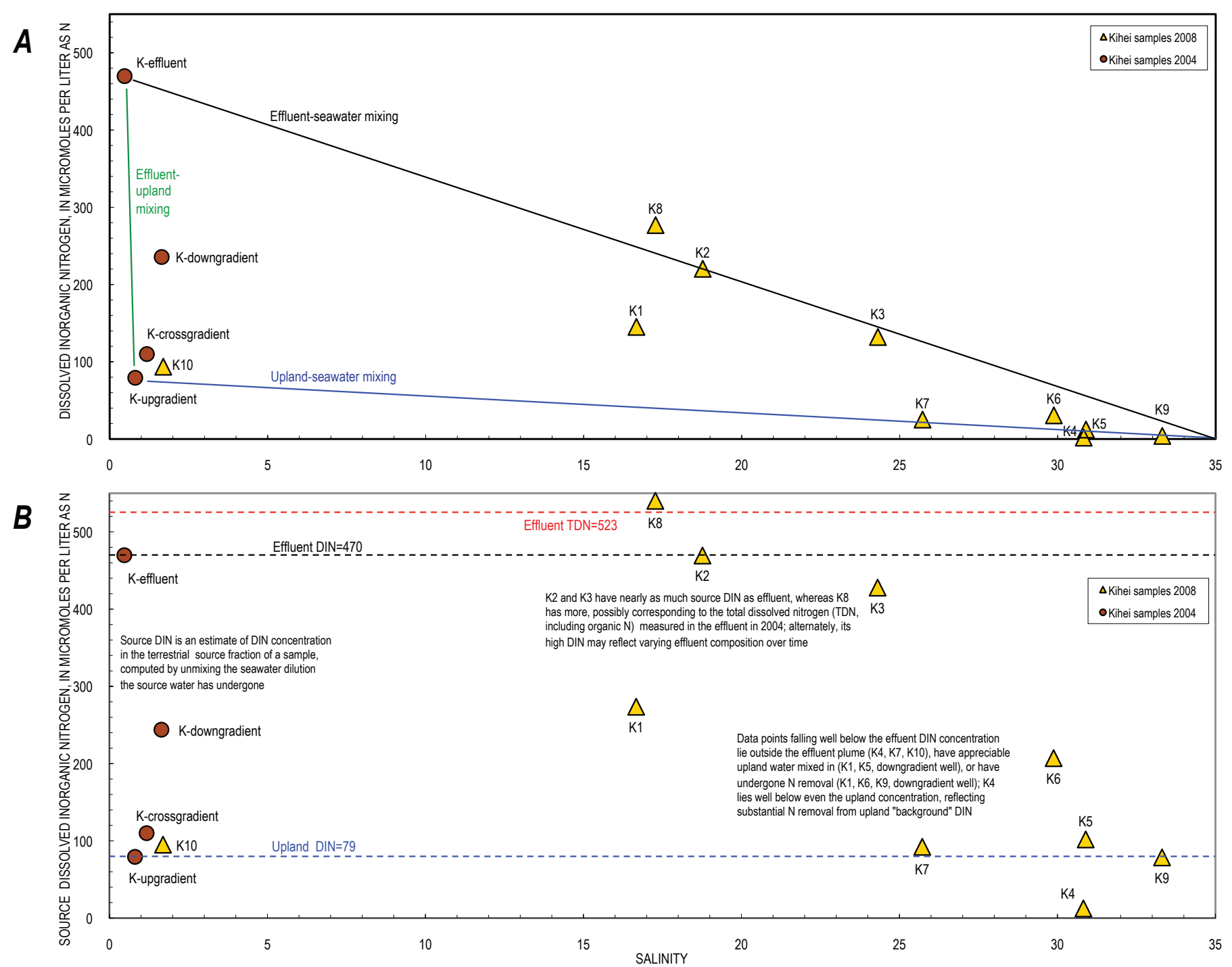

Figure 33. Seawater mixing plots for $(A)$ dissolved inorganic nitrogen (DIN), and ( $B$ ) source DIN (adjusted for seawater dilution) in water samples at Kihei, Hawaii. 
denitrification trends have been drawn from effluent (pink arrow) and upland water (blue arrow), both at a slope of 1:2 $\left(\delta^{18} \mathrm{O}: \delta^{15} \mathrm{~N}\right)$ found to be representative in a number of studies (Kendall and McDonnell, 1998). Computed mixing lines have small dots at 1-percent increments to distinguish them as computed and not simply hand-drawn; increment spacing is nonlinear because compositions are concentration-weighted in equation 4 . In the bottom panel $(B)$ of figure 34 , source DIN (table 3) is plotted on the Y-axis, while $\delta^{15} \mathrm{~N}$ of dissolved nitrate is retained on the $\mathrm{X}$-axis. Both plots can be interpreted in terms of land-derived nitrogen, disregarding seawater for two reasons: (1) the upper panel portrays isotopic compositions of dissolved nitrate, which is overwhelmingly terrestrial in this setting (local seawater contains so little nitrate that it will have negligible influence on these samples); and (2) the lower panel plots source DIN from seawater unmixing equation 1 , thereby removing seawater as a variable (if the unmixing calculation truly works as intended).
In fig. 34A, samples most likely to have evolved through effluent denitrification are $\mathrm{K} 9$ and the downgradient well (the isotopically heaviest sample). Recalling that the downgradient well contained roughly three-quarters effluent and one-quarter upland water, an unmixing calculation (equation 3) was made to remove the upland-water dilution and estimate the "denitrified-effluent fraction" in the sample. This is a hypothetical end member with slightly heavier isotopic composition (orange circle) than the downgradient well, which then plots at the 75-percent point on a mixing line between the denitrified end member and upland water (orange line), as expected. On figure $34 B$, a denitrification trend (pink line) was fit to the denitrified end member using fractionation equation 5 and an enrichment factor $\varepsilon=-22$. From this same equation, the denitrified end member is calculated to have undergone 37 percent denitrification (63 percent of effluent DIN remaining).

Although samples K1, K2, K3, K6, and K8 plot along the orange mixing line in fig. $34 A$, this appears to be only by

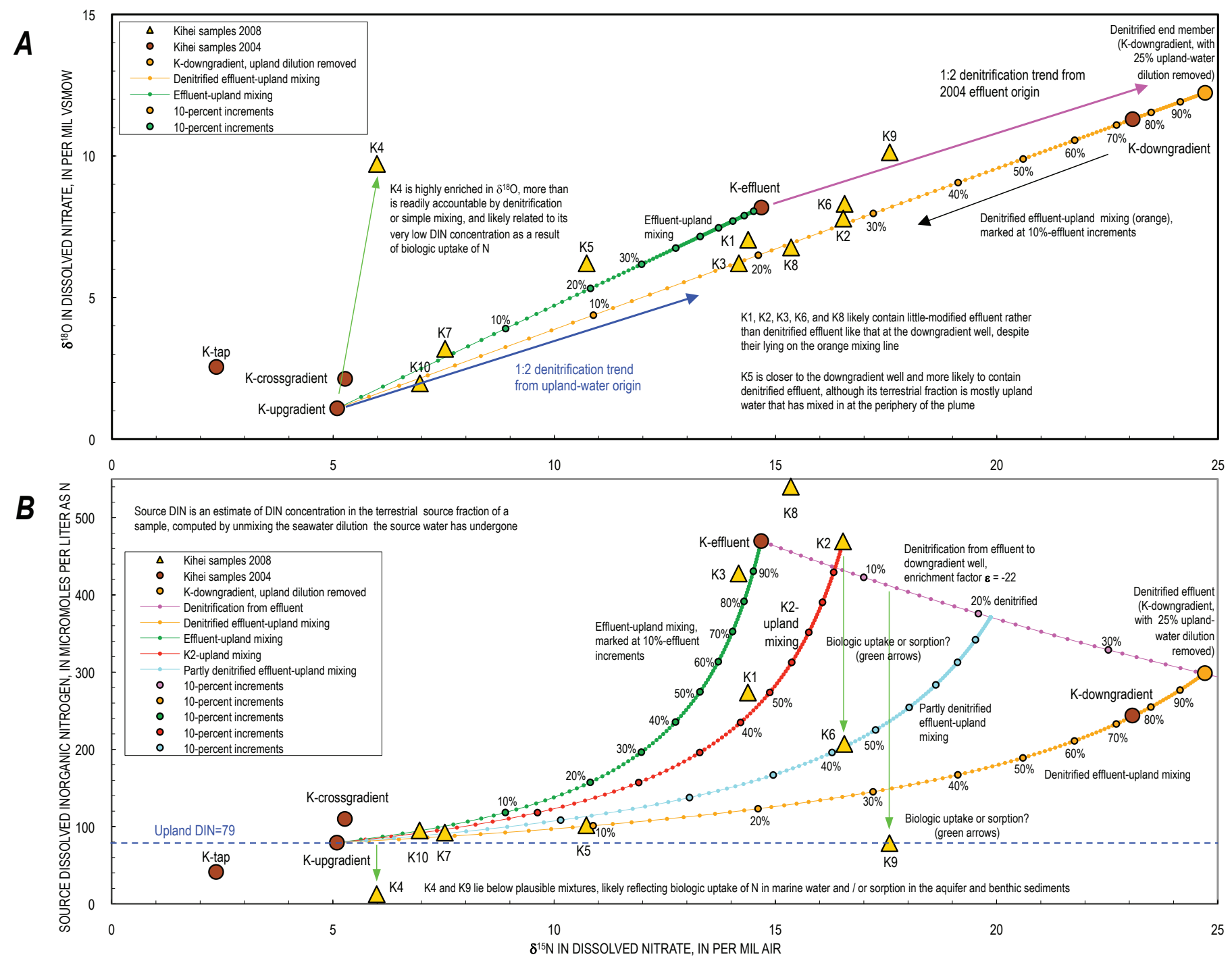

Figure 34. Isotope mixing and evolution plots for $(A) \delta^{18} 0$ versus $\delta^{15} \mathrm{~N}$ of dissolved nitrate, and $(B)$ source DIN versus $\delta^{15} \mathrm{~N}$ of dissolved nitrate in water samples at Kihei, Hawaii. 
coincidence, as they do not plot along the same mixing line in fig. $34 \mathrm{~B}$ and probably are not mixtures of denitrified effluent and upland water. Instead, they appear to contain little-modified effluent differing only slightly from the sampled 2004 effluent composition. K8 contains more source DIN than the 2004 effluent, as mentioned earlier, and K2 appears to be only slightly denitrified, not nearly as much as the downgradient well sample. K2 and K8 lie right next to each other on the map (fig. 9), and together these two samples suggest a plume core of little-modified effluent.

Samples K1, K3, and K6 appear to be variously diluted with upland water, which is expected given their locations at the plume periphery. K3 is consistent with slight dilution of the sampled effluent. Samples K1 and K6 lie close together on the map and may have evolved from the nearby K2 composition: K1 by simple upland-water dilution (fig. 34B, red line) and K6 perhaps largely by biologic uptake (fig. 34B, green arrow) lowering its DIN from that at K2. An alternate hypothesis for K6 is simple upland-water dilution from a 21-percent denitrified effluent composition (blue line). Different evolution mechanisms for samples as close together as K1 and K6 is not out of the question, given the probable heterogeneity of the coastal plain sediments (mud, sand lenses, coralline rubble) through which the plume discharges. Sample K9 offers additional support for biologic uptake: its areal position near the core of the plume largely rules out upland-water dilution as a means of lowering its DIN, leaving the more likely processes of partial denitrification (fig. 34A) and biologic uptake (fig. 34B). Another possibility is that the seawater unmixing calculation used to estimate "source DIN" does not work as well as intended, particularly for such a saline sample (95 percent seawater).

Sample K5 at the plume periphery is a plausible mixture of upland water and 10 percent denitrified effluent of a composition best exemplified by the nearby downgradient well. Samples K7 and K10 plot close to upland water at the upgradient and crossgradient wells, although they may have undergone slight denitrification from upland parent compositions (fig. 34A). Sample K4 from Waipuilani Park far to the north (fig. 9) is so highly enriched in ${ }^{18} \mathrm{O}$ (fig. 34A) and so much lower in source DIN than its expected parent upland water (fig. 34B) that it appears to require a different source of water or modification by unknown processes.

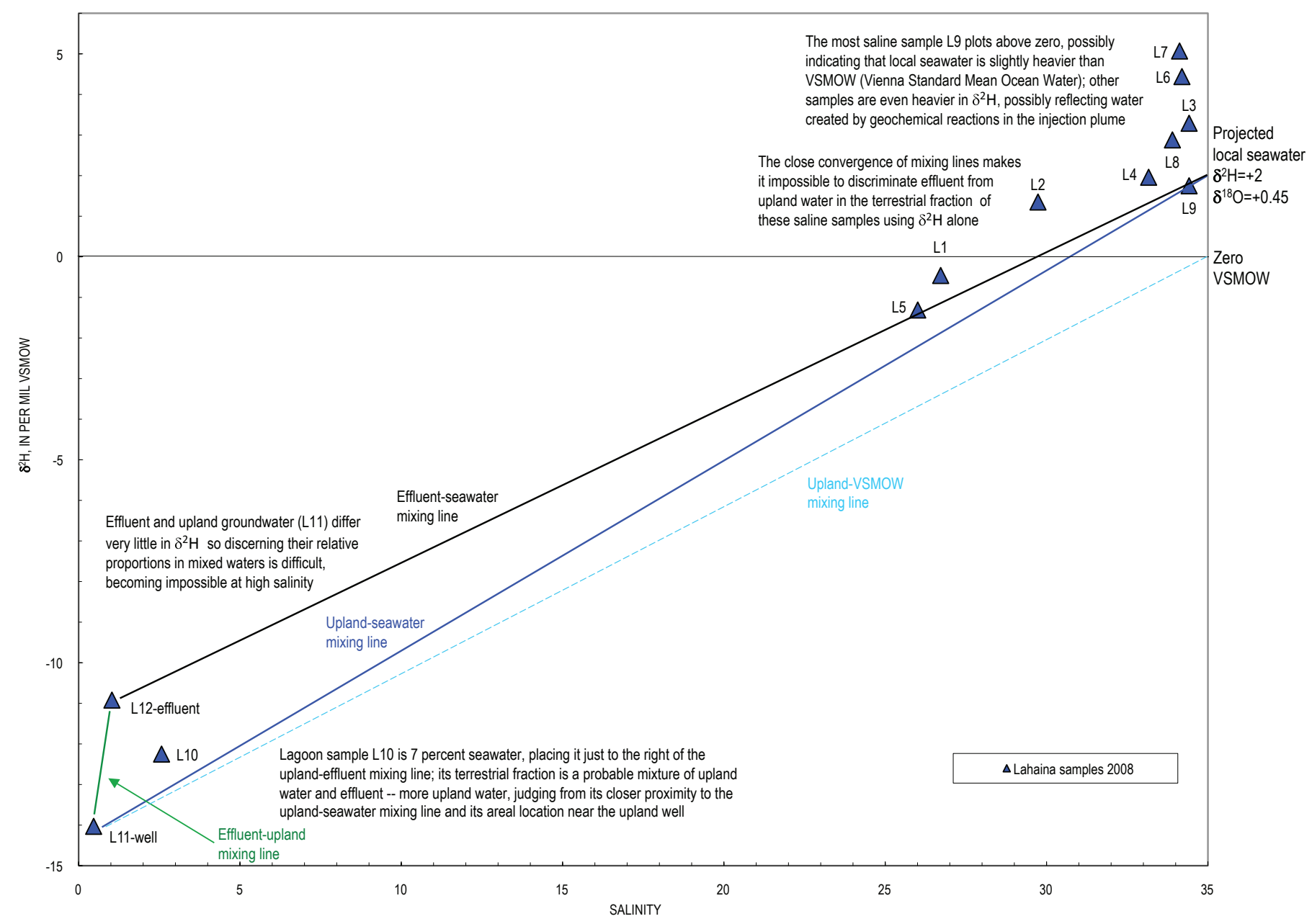

Figure 35. Seawater mixing plot showing $\delta^{2} \mathrm{H}$ versus salinity at Lahaina, Hawaii. 
We have listed a few possible interpretations of nutrient evolution in the coastal aquifer and benthic sediments containing the injected effluent. All interpretations hinge on DIN and $\delta^{15} \mathrm{~N}$ of sources being representative generally, including the effluent sample taken in 2004 and compared against the 2008 marine-water samples collected along the shoreline. Time-variability of DIN or $\delta^{15} \mathrm{~N}$ in the injected effluent would modify these interpretations.

\section{Mixing and Effluent Evolution at Lahaina}

At Lahaina, water isotopes were less useful than at Kihei for discriminating effluent from upland groundwater because of the small isotopic separation between the two (fig. 35). $\delta^{2} \mathrm{H}$ only differed by 3.1 per mil between upland groundwater and effluent, and only lagoon sample L10 was fresh enough to be constrained as a mixture more heavily weighted by upland water than effluent. Remaining samples are much more saline and plot well to the right side of the seawater mixing plot. A local seawater composition was projected from the most saline sample L9 (98 percent seawater), assuming its fresh fraction is mostly upland water; the resulting local seawater composition is $\delta^{2} \mathrm{H}=+2$ per mil and $\delta^{18} \mathrm{O}=+0.45$ per mil, not much different from the local seawater composition estimated for Kihei. All samples plot above an upland-VSMOW mixing line, and most plot above the local seawater mixing lines and show a fairly large spread in $\delta^{2} \mathrm{H}$ values, the significance of which is unclear.

Seawater mixing plots for nutrients at Lahaina (fig. 36) show some notable departures from conservative mixing among end members. Submarine spring samples L1, L2, and L5 are known to be in the heart of the effluent plume from pharmaceuticals and heavy $\delta^{15} \mathrm{~N}$. They plot below the effluent-seawater mixing line for DIN (upper panel, figure 36A), which is expected from denitrification in the plume. But their orthophosphate concentrations (lower panel, figure 36B) plot well above the effluent seawater mixing line, suggesting some
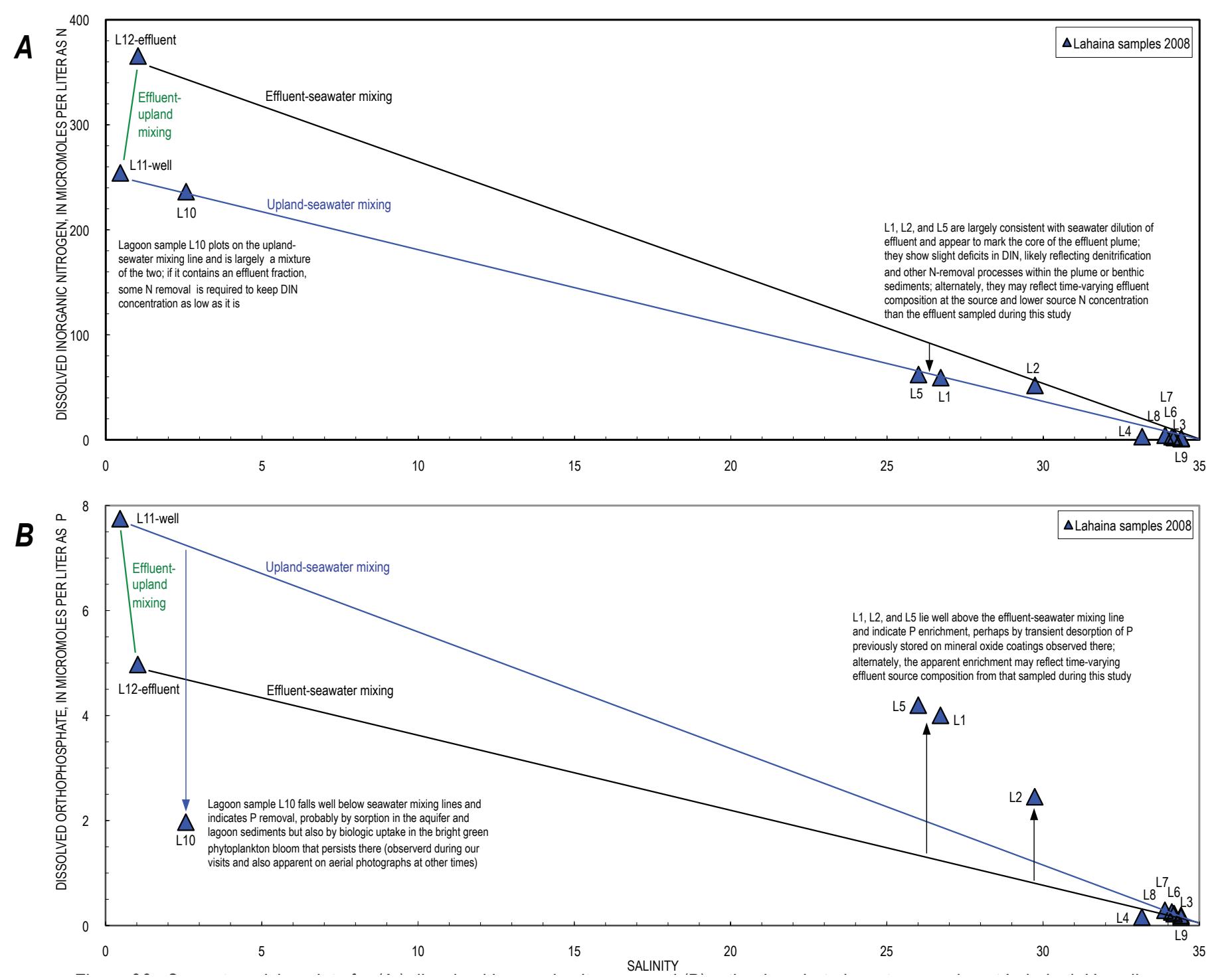

Figure 36. Seawater mixing plots for $(A)$ dissolved inorganic nitrogen, and $(B)$ orthophosphate in water samples at Lahaina, Hawaii. 
addition of $\mathrm{PO}_{4}$ over effluent composition (possibly related to mineral precipitates observed on bed sediments during collection of these samples, as previously detailed), or that they originated from effluent having a higher $\mathrm{PO}_{4}$ concentration than the sampled effluent. Lagoon sample L10 plots on the upland-seawater mixing line for DIN (fig. 36A), which at first glance suggests it is a simple conservative mixture of upland groundwater and seawater. However its heavy $\delta^{15} \mathrm{~N}$ value of about +13 per mil (table 4 and fig. 29) would suggest some denitrification has occurred, which would drop it below the mixing line. The most plausible hypothesis is that sample L10 contains a fraction of denitrified effluent that keeps it on the upland-seawater mixing line while at the same time having $\delta^{15} \mathrm{~N}$ heavier than upland water. The orthophosphate concentration of lagoon sample L10 falls well below seawater mixing lines, requiring substantial loss of $\mathrm{PO}_{4}$ beyond conservative seawater mixing (fig. 36B). As discussed earlier in the section on $\mathrm{N}$ :P ratios, some amount of this loss may occur by biologic uptake in the lagoon phytoplankton bloom, but $\mathrm{P}$ depletion in the aquifer and pond sediments appears to be required as well.

These interpretations are reinforced by unmixing seawater dilution to obtain source DIN concentrations (fig. $37 B$ and table 4). Samples L1 and L5 source DIN concentrations plot well below effluent concentration, indicating greater $\mathrm{N}$ loss than in sample L2, which plots only slightly below effluent. Lagoon sample L10 has essentially the same source DIN concentration as the L11 upland well sample, consistent with L10 plotting on the upland-seawater mixing line in the seawater mixing plot (upper panel, figure 37A). Saline samples at the far right side of figure $37 B$ plot well below effluent and upland water. This indicates that they have undergone substantial loss of $\mathrm{N}$ by denitrification, ammonium sorption, and biologic uptake, or that the seawater unmixing calculation has not worked entirely as intended for these more saline samples.
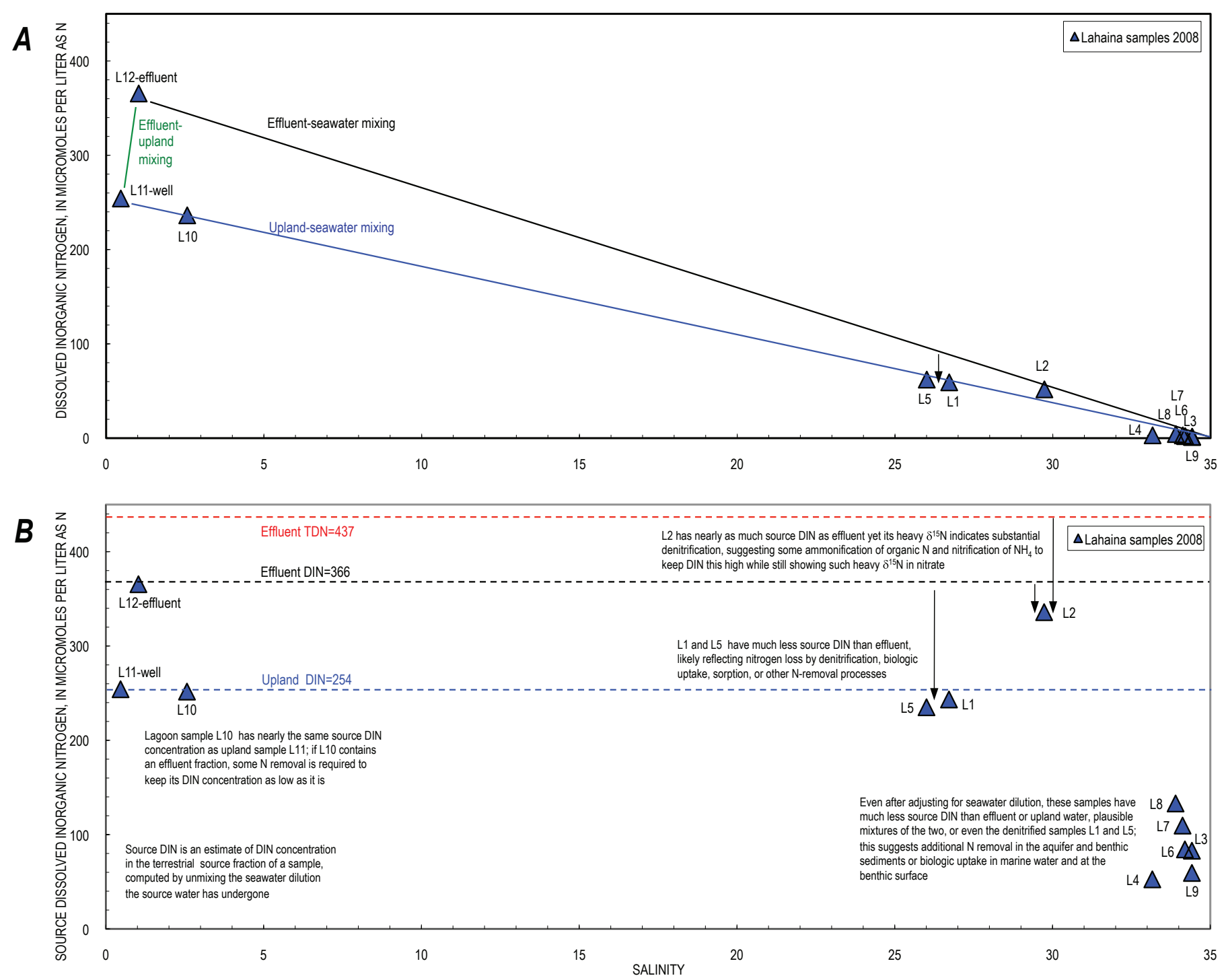

Figure 37. Seawater mixing plots for $(A)$ dissolved inorganic nitrogen (DIN), and (B) source DIN (adjusted for seawater dilution) in water samples at Lahaina, Hawaii. 
Stable-isotope evolution in dissolved nitrate at Lahaina follows expectable mixing and denitrification trends for some samples, but the more saline samples plot well off of expected trend lines (fig. 38). In the upper panel (fig. 38A), hypothetical denitrification trends have been drawn from effluent (pink arrow) and upland water (blue arrow), both at a slope of 1:2. Submarine spring samples L1, L2, and L5 plot in a position roughly consistent with the effluent denitrification trend and are believed to represent conditions in the core of the injection plume. The denitrified effluent-upland mixing line has been computed (equation 4) between upland well sample L11 and the denitrified composition of sample L5 (orange line, figure $38 \mathrm{~A}$, with symbols at 1 -percent mixing increments). The rationale here is that effluent is denitrified within the core of the injection plume and then mixes with upland groundwater at the outer periphery of the plume. Samples L8 and L10 plot along the mixing line, consistent with this hypothesis, and also possibly sample L4. However, L4 isotopic composition could also have evolved by slight denitrification from upland groundwater (blue arrow) without containing any effluent. Samples L3, L6, L7, and L9 plot well off of the expected mixing and denitrification trends and suggest some other process at work, perhaps biologic uptake (green arrows, drawn at the theoretical 1:1 slope for uptake) or some unidentified aquifer process taking place more in the periphery of the plume than at its core.

In the lower panel (fig. 38B), plotting source DIN against $\delta^{15} \mathrm{~N}$ yields a similar pattern of some samples plotting along expected trends and others plotting well off trend. A denitrification trend has been fit to sample L5 with an enrichment factor $\varepsilon=-38$, resulting in an estimate that sample L5 is 36 percent denitrified (64 percent of effluent DIN remaining), nearly the same as the most denitrified sample at Kihei (the downgradient well, with upland-water dilution removed). Sample L2 is difficult to explain: it has a heavy $\delta^{15} \mathrm{~N}$ value, essentially the same as sample L5 (roughly +40 per mil; table 4 and fig. 29) and indicating the same degree of denitrification,
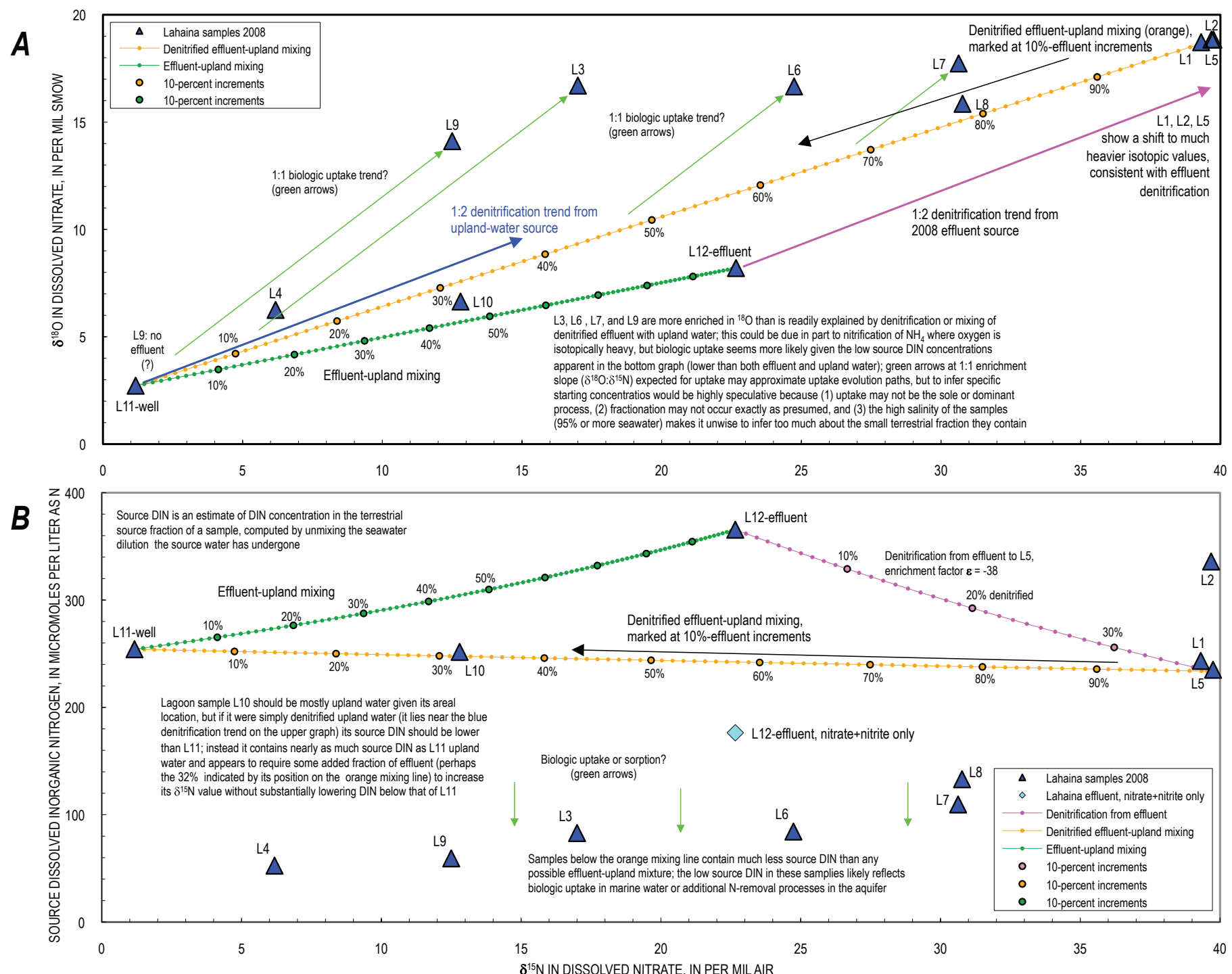

Figure 38. Isotope evolution and mixing plots for $(A) \delta^{18} 0$ versus $\delta^{15} \mathrm{~N}$ of dissolved nitrate, and $(B)$ source DIN versus $\delta^{15} \mathrm{~N}$ of dissolved nitrate in water samples at Lahaina, Hawaii. 
but its source DIN is closer to effluent than to L5 source DIN and has $105 \mu \mathrm{M}$ more ammonium than L5 (table 4). This could reflect the presence of effluent with higher DIN than the sampled effluent, possibly by conversion of organic nitrogen in effluent TDN to inorganic form. However, because L2 and L5 were collected at the same site but hours apart, the difference may simply have to be attributed to sampling variability in an environment of short-term wave and tide forcing. Notably, the nitrate+nitrite concentration in effluent is plotted as a light blue diamond on figure $38 \mathrm{~B}$. This shows the necessity of plotting DIN rather than nitrate on these plots, else many of the other samples would have more nitrate than the effluent does, despite showing very heavy $\delta^{15} \mathrm{~N}$ compositions indicating substantial loss of $\mathrm{N}$ to denitrification.

Lagoon sample L10 falls exactly on the denitrified effluent-upland mixing line (orange line) on figure $38 \mathrm{~B}$, providing additional support for interpreting it as mixture of upland groundwater and denitrified effluent (in a 68-32 percent blend, according to the mixing computation and plot). Reclaimed water is used to irrigate the golf course in that area and might be questioned as the source of an effluent signature there. However, L10 falls exactly on the denitrified effluentupland mixing line (orange) rather than the effluent-upland mixing line (blue) on figure $38 B$, and in the absence of further work the denitrified-effluent hypothesis is favored for now. If reclaimed water were the source of the apparent effluent signature at L10, the data point aligns roughly with a 45-percent-effluent composition on the effluent-upland mixing line, implying nearly equal parts reclaimed water and upland water in the sample.

Remaining samples plot well below the mixing line, indicating additional nitrogen loss beyond that attributable to seawater dilution or the 36 percent denitrification of sample L5. There could be additional denitrification in the aquifer, perhaps in the plume periphery, but this cannot be known without a representative sample of that end member. Biologic uptake in the marine water column and benthic sediments may cause the low source DIN concentrations, but additional porewater and marine chemistry would be needed to confirm this, and the value of the present dataset is simply to point out the anomaly from expected trends. Taken in their entirety, however, these data indicate that (1) there is substantial denitrification in the Lahaina injection plume, as there was at Kihei; and (2) there is evidence for an effluent fraction as far south as lagoon sample L10 at Black Rock.

\section{Comparison of Injection Plumes at Kihei and Lahaina}

The Kihei and Lahaina wastewater injection plumes were successfully detected with our wading surveys employing a multitracer strategy. Prior simulation at Kihei (Hunt, 2007) provided a modeled plume extent against which survey results can be compared. No model of similar sophistication has been applied to the Lahaina injection wells, and past attempts at plume detection were inconclusive until the recent macroalgae surveys of Dailer and others (2008). Results of our wading surveys now allow a more complete conceptual picture to be drawn for the Lahaina plume. Nutrient and isotopic results from our surveys also allow a comparison of nutrient natural attenuation apparently occurring within the plumes.

\section{Extent of the Injection Plumes}

The injection plume at Kihei was found to coincide well with the modeled plume of Hunt (2007), as illustrated by $\delta^{15} \mathrm{~N}$ in algae and water samples (fig. 39; only a select few water samples are shown to extend coverage beyond the algal transect). The plume is well bounded on the south by beach spring sample K10, which has a predominantly upland-water character. The plume is less well defined to the north, where $\delta^{15} \mathrm{~N}$ values oscillate somewhat, but it probably does not extend much beyond Welekahao Road, if at all. As shown earlier, this confirmatory plume delineation by $\delta^{15} \mathrm{~N}$ is also supported by detections or elevated concentrations of pharmaceuticals, waste indicator compounds, and nutrients.

The injection plume at Lahaina was also detected convincingly (fig. 40), although the apparent locus of effluent discharge is not directly offshore from the injection wells but is farther south than expected. We did not detect a strong effluent signature along Honokowai Point, although our surveys were inshore in shallow, wadeable water depths and our results do not preclude effluent discharge farther offshore, for example seaward of the fringing reef. However, prior surveys for injected rhodamine dye and nutrients off Honokowai Point failed to turn up compelling indications of effluent discharge there (Tetra Tech, 1994; Bourke, 1996; Dollar and Andrews, 1997).

The apparent indirect path that effluent takes from the Lahaina injection wells requires some geologic feature or anomaly for explanation. One possibility, shown in figure 40, is a probable buried valley along an ancestral course of Honokowai Stream. Over time, stream channels migrate back and forth where they let out onto flatter terrain in an alluvial fan. Currently, the modern Honokowai Stream flows north of Honokowai Point. A prominent reentrant in offshore bathymetry contours may mark a former southward swing of Honokowai Stream, perhaps during the last glacial lowstand of sea level or at some time since its return to present level about 5,000 years before present (Grossman and Fletcher, 1998). Drowned stream valleys in Hawaii are known to act as hydraulic barriers in the permeable volcanic-rock aquifers: the valleys cut into the underlying aquifer, and subsequent drowning by rising sea level leaves a fill of low-permeability sediments, such as finegrained muds and clay-rich weathered alluvium (Palmer, 1927; Ferrall, 1976; Oki, 2005). Such a valley fill at Honokowai may essentially "wall off" the effluent plume from migrating north and directly west from the Lahaina injection wells. Honokowai Stream has carved the upper part of the volcanicrock aquifer (Wailuku Volcanics, in green on fig. 40) into two 


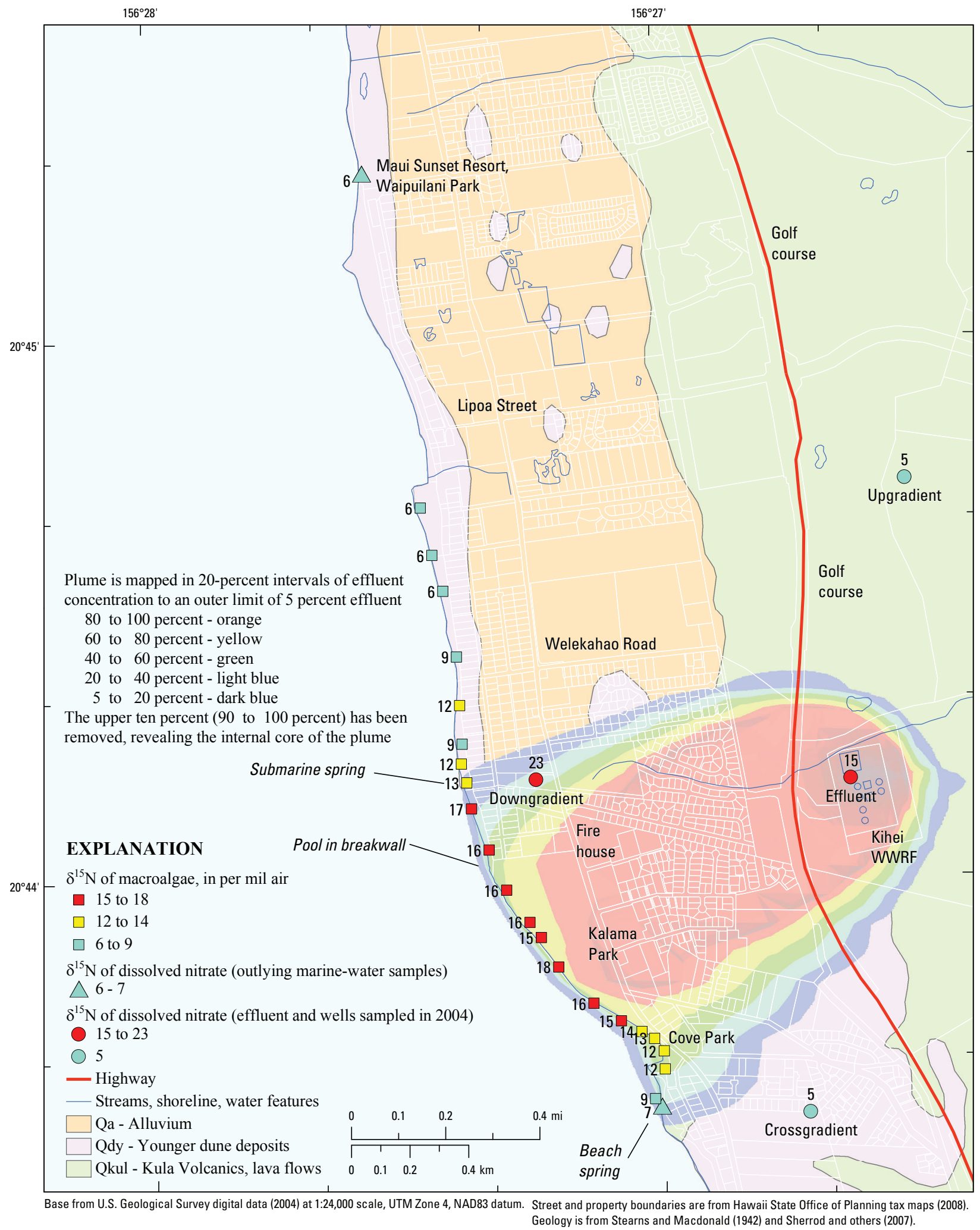

Figure 39. Map showing correspondence between $\delta^{15} \mathrm{~N}$ and the modeled injection plume of Hunt (2007) at Kihei, Hawaii. 


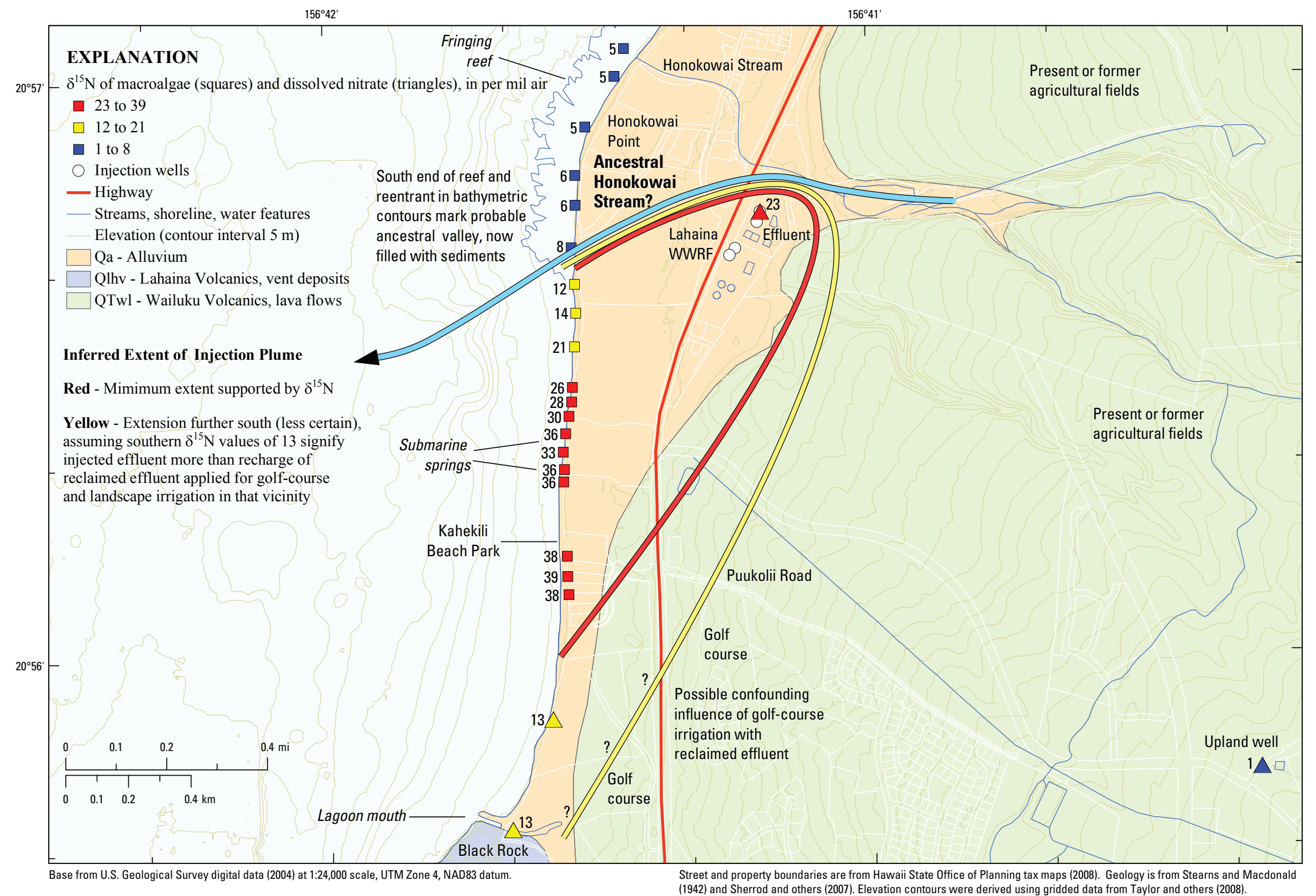

Figure 40. Map showing injection plume inferred from $\delta^{15} \mathrm{~N}$, and southward diversion of the plume by a probable buried ancestral valley at Lahaina, Hawaii. 
lobes, north and south of the valley. Effluent injected south of the postulated valley-fill barrier will rise buoyantly along the "ceiling" of the south lobe, whose geometry will direct it to the southwest, where it will continue to rise buoyantly through the overlying sediments to discharge at the shore. Another possibility is that effluent rises into sediments near the injection wells and is directed southwest within buried stream channels of permeable alluvium or into a sedimentary formation with a longshore orientation, such as a buried coral reef. Regardless of the exact geologic cause, stable-isotope evidence for an effluent fraction in lagoon sample L10 merits drawing the apparent plume as far south as Black Rock, speculatively (fig. 40, yellow arc). If reclaimed-water irrigation is the source of the effluent signature in that area instead, then the smaller plume extent (fig. 40, red arc) is supported, at a minimum, by the $\delta^{15} \mathrm{~N}$ data. For irrigation to be the source of the effluent signature in the south, turf irrigation would have to be at such a heavy rate that it generates a return-recharge flux volumetrically comparable to the ambient flow of upland groundwater, which is about $5 \mathrm{Mgal} /$ day per mile of coast under natural conditions (Shade, 1996).

Our inshore survey results don't preclude effluent discharge farther offshore at Kahekili Beach. Effluent may be detectable in deeper water, and scuba reconnaissance and porewater sampling surveys might be helpful in mapping the offshore extent of effluent discharge. For the time being, our surveys illustrate that effluent discharge is mappable as a distinct spatial pattern next to the beach.

\section{Comparison of Nutrient Attenuation at Kihei and Lahaina}

Once injected into the aquifer at Kihei and Lahaina, wastewater nutrients are subject to natural attenuation by various processes, such as sorption and microbial breakdown. For nitrogen, the major attenuation process envisioned to be occurring in the effluent plumes is denitrification, for which fractionation trends were fitted to the data (fig. 34 and fig. 38). These fractionation trends are reproduced in fig. 41, this time showing the fraction of initial effluent nitrogen lost to denitrification. At Kihei and Lahaina, the maximum denitrification in any sample was 37 and 36 percent, respectively. At Kihei this was for a hypothetical denitrified effluent inferred from the downgradient well by subtracting upland-water dilution. At Lahaina it was in sample L5, although L1 and L2 isotopic compositions result in nearly the same estimate of denitrification degree. At Kihei, there was also evidence of a lesser degree of denitrification in some samples. Sample K9 has an isotopic composition consistent with only 12 percent denitrification, and samples $\mathrm{K} 2$ and $\mathrm{K} 8$ appear to be even less denitrified (fig. 34). So, although some samples indicate substantial loss of nitrogen, the degree of denitrification estimated by Hunt (2007) from the single downgradient-well sample available at the time has now been revealed as not representative of the Kihei plume as a whole (it probably reflects localized conditions more toward the lateral periphery of the plume and in the coastal-plain sediments in which the well is open). In addition to identifiable denitrification losses, there also was evidence for further nitrogen loss by biologic uptake in samples at both locales. Estimation of total nutrient delivery to the coastal ecosystem is therefore a daunting task, and further refinement of nutrient mass fluxes is beyond the scope of the present study.

Phosphorus attenuation first highlighted by Hunt (2007) is supported by our wading samples at Kihei, but not at Lahaina. After unmixing effects of seawater dilution at Kihei (table 3), the maximum source orthophosphate concentrations in samples $\mathrm{K} 2$ and $\mathrm{K} 8$ were still less than one-third the sampled 2004 effluent concentration, not quite the ninefold reduction cited by Hunt for the downgradient well, but still a substantial reduction. At Lahaina (table 4), maximum source orthophosphate concentrations at submarine spring samples L1, L2, and L5 were actually greater than sampled effluent concentration, by a factor of about 3 . This could indicate some temporary desorption of phosphorus from mineral coatings or that the sampled effluent is not representative generally. Notably, very few of the Lahaina water samples had source orthophosphate concentrations much less than the sampled effluent. A tentative conclusion is that phosphorus attenuation at Lahaina appears to be less active than at Kihei.

\section{Summary and Conclusions}

Municipal wastewater injection plumes were successfully detected in the ocean by nearshore wading surveys at Kihei and Lahaina, Maui. Treated wastewater presence was confirmed by multiple "inherent" wastewater tracers, the most conclusive being pharmaceuticals, organic waste indicator compounds, and heavy $\delta^{15} \mathrm{~N}$. Denitrification is active in both plumes, raising $\delta^{15} \mathrm{~N}$ values above those in effluent and providing more contrast with surrounding waters, particularly at Lahaina, where $\delta^{15} \mathrm{~N}$ values nearly double that of effluent were measured at submarine springs and in algae samples.

The pharmaceuticals carbamazepine and sulfamethoxazole were detected at both locales and appear to be particularly persistent through the waste-treatment process and upon release to the environment and subsequent migration through the aquifer. At Lahaina, wastewater presence was further confirmed at submarine springs by tribromomethane, two musk fragrances, a fire retardant, and a plasticizer compound that were all also detected in sampled effluent at the treatment plant. Tribromomethane was also detected in the single Kihei marine-water sample for which it was analyzed. Caffeine detections appear not to be related to treated wastewater effluent but may instead originate from swimmers or be a first indication of raw sewage sources, such as chronically leaking sewer lines or remnant cesspools.

There is evidence of natural attenuation of nutrients within the effluent plumes, presumably from sorption and bacterial degradation in the aquifer, and there is also evidence in 


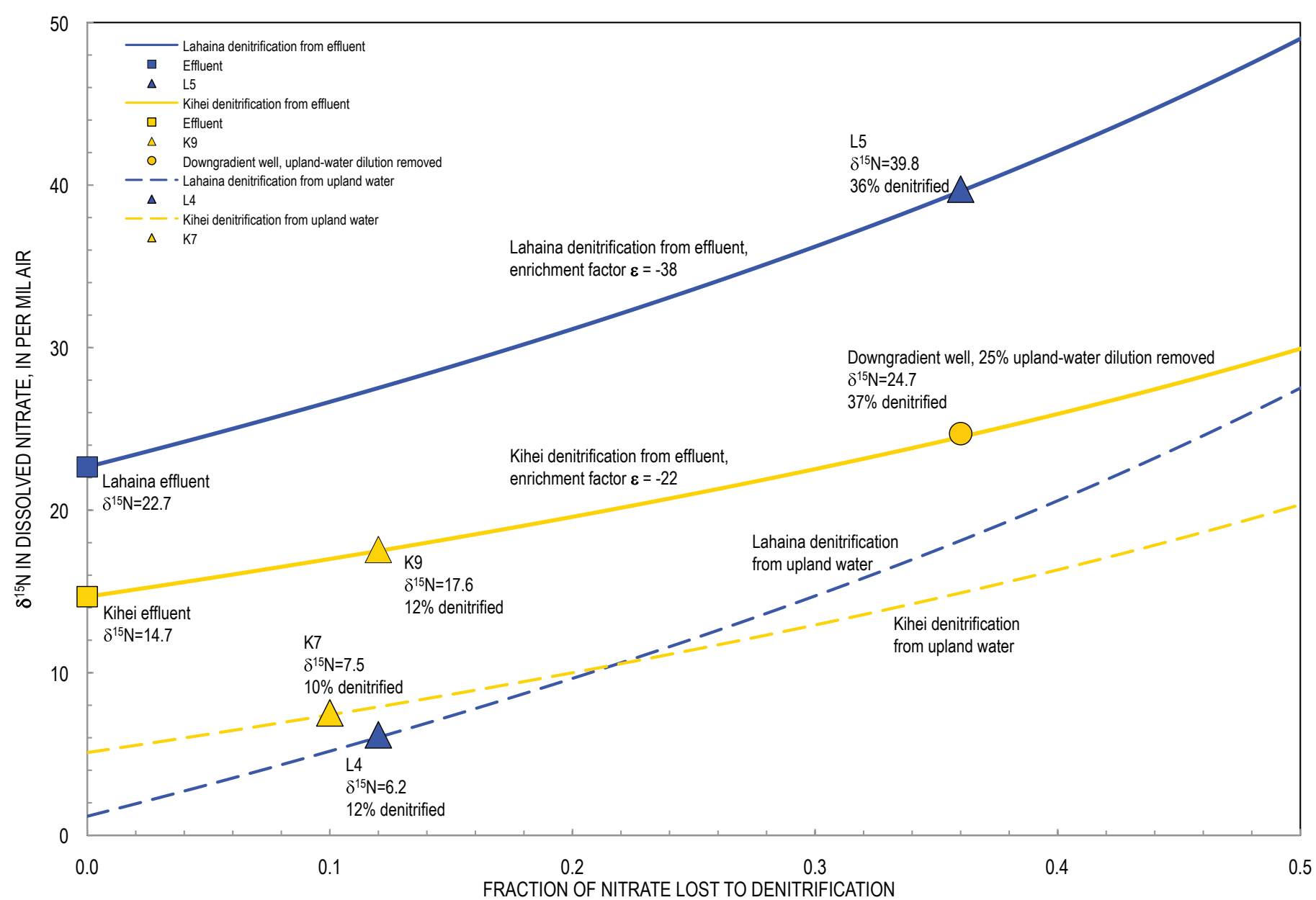

Figure 41. Graph comparing denitrification of effluent and upland groundwater at Kihei and Lahaina, Hawaii.

some marine-water samples of biologic uptake of nutrients in the marine water column or benthic sediments. Despite these nutrient-reducing mechanisms, the effluent plumes constitute large nutrient fluxes to the nearshore environment. Nutrient concentrations are quite high, even in our marine-water samples, although concentrations were highest in the freshest samples and can be expected to decrease farther offshore with further seawater dilution of the emerging groundwaters. Samples from upland wells at Lahaina illustrate that fertilizerderived nutrient concentrations in groundwater can be as high as - or higher than - those in treated wastewater effluent. The effluent plumes are not the sole source of nutrients discharging to the ocean on Maui, of course. Groundwater beneath fertilized fields is similarly high in nitrogen as the effluent, and groundwater nitrogen concentrations beneath even undeveloped forests are two to three orders of magnitude higher than in low-nutrient seawater. Phosphorus concentration is considerably higher in effluent than in forest or agricultural groundwaters, however.

\section{Study Limitations and Opportunities for Future Research}

The numbers of samples submitted for laboratory analysis at each locale were small: about 20 algae samples and 10 water samples, of which only 4 were analyzed for pharmaceuticals and 1 for waste indicator compounds. The small number of samples was adequate for the primary objective of the study, which was detection of wastewater effluent in the ocean. More complex objectives are addressed in this report (delineation and mapping of the effluent plumes; characterization of plume chemistry and nutrient concentrations in plume discharge; inferring geochemical processes and nutrient attenuation in the plumes); however, data adequacy generally decreases with increasing complexity and many of the interpretations should be considered highly tentative. The very small number of pharmaceutical and waste-indicator analyses was a cost consideration, and resampling for a larger number of these analyses would be desirable for a more thorough 
plume characterization. Results of the present study provide a good basis for designing a followup sampling strategy.

The plumes were mapped and delineated quite well by nitrogen isotopes: the core of each plume is marked by heavy $\delta^{15} \mathrm{~N}$ values that decrease to lighter values at the plume periphery. Outer limits of the plume were well defined at Kihei, but at Lahaina the southern limit was less distinct $\left(\delta^{15} \mathrm{~N}\right.$ remained elevated at +13 per mil in the southernmost samples, and there may be a confounding influence from golf-course irrigation with reclaimed effluent). A limited amount of sampling south of Black Rock might constrain the southern limit of the Lahaina plume with more certainty, or it might give more indication of an irrigation source of elevated $\delta^{15} \mathrm{~N}$ in that vicinity.

Our surveys demonstrate that effluent discharge is mappable as a distinct spatial pattern next to the beach but do not preclude effluent discharging farther offshore. Scuba reconnaissance and porewater sampling might be helpful in mapping the offshore extent of effluent discharge, and these could be preceded and guided by a geophysical survey in which an electrical resistivity "streamer" is towed back and forth offshore by boat to map fresher water zones on the sea bottom that could be prioritized for porewater sampling (Mannheim and others, 2002; Johnson and White, 2007; Nyquist and others, 2008).

Our surveys were instantaneous in time (representing the instant our trolling instrument passed by or a water sample was taken), whereas effluent chemistry at the treatment plant varies somewhat day to day and conditions at the ocean bottom fluctuate interannually, seasonally, over the daily tidal cycle, and with wave forcing at periods on the order of 12-20 seconds. Only by recording information over such time scales can that variability be characterized or "average" conditions determined. Although variability at these time scales likely influences our results, there is no way to know how or to what degree without detailed measurement and analysis beyond the scope of our study.
Natural attenuation of nutrients in the effluent plumes is evident in a few water samples, and this topic may warrant further "process-oriented" study by geochemists specializing in nutrient cycling and fate in wastewater plumes. Two new multidepth nests of monitor wells in the Kihei plume can be used for this purpose, and it may be worthwhile to consider installation of additional monitor wells and/or porewater sampling transects along the beach or across the plumes at distances inland with a "push-sampler" rig.

A current assessment of nutrient loads from various land uses could be an aid in making sound nutrient-management decisions and should include forest, agricultural, and urban nutrient loads as well as injected-effluent nutrient loads. We sampled only one well at Lahaina to characterize nutrient concentrations attributable to fertilizers; certainly more wells should be sampled if a fertilizer nutrient flux were to be estimated. Effluent nutrient fluxes can be estimated in similar fashion to Soicher and Peterson (1997) and Hunt (2007), although better characterization of both plumes would be desired first, because some of our water samples indicated substantial natural attenuation of nutrients and other samples indicated little attenuation. One would want to improve estimates of the average or representative nutrient concentrations that would be used in nutrient-flux calculations.

Marine scientists are continuing with their evaluation of the effects of effluent discharge on the marine ecosystem. Results of our study provide a clearer picture of where these two plumes on Maui discharge along the shore and may help marine researchers target specific sites or tailor their observation transects to what is known thus far. Further study of the plumes and their effects will benefit from interdisciplinary interaction among marine and terrestrial scientists, and this ideally would include collaboration at the study-design stage. 


\section{References Cited}

Atkinson, S., Atkinson, M.J., Tarrant, A.M., 2003, Estrogens from sewage in coastal marine environments: Environmental Health Perspectives, v. 111, no. 4, p. 531-535.

Bailey-Brock, J.H., 1976, Habitats of tubicolous polychaetes from the Hawaiian Islands and Johnston Atoll: Pacific Science, v. 30, no.1, p. 69-81.

Barber, L.B., Meyer, M.T., LeBlanc, D.R., Kolpin, D.W., Bradley, P.M., Chapelle, F.H., and Rubio, F., 2008, Subsurface fate and transport of sulfamethoxazole, 4-nonylphenol, and 17B-estradiol, in Trefry, M.G., ed., Groundwater quality 2007-securing groundwater quality in urban and industrial environments: International Association of Hydrological Sciences IAHS Redbook, IAHS Publ. 324, p. 133-139.

Bohlke, J.K., Smith, R.L., and Miller, D.N., 2006, Ammonium transport and reaction in contaminated groundwater-application of isotope tracers and isotope fractionation studies: Water Resources Research, v. 42, W05411, 19 p.

Bourke, R., 1996, Maui algae bloom studies - distribution and abundance: Honolulu, Hawaii, Oceanit Laboratories, Inc.

Buerge, I.J., Poiger, T., Muller, M.D., and Buser, H.-R., 2006, Combined sewer overflows to surface waters detected by the anthropogenic marker caffeine: Environmental Science and Technology, v. 40, no. 13, p. 4096-4102.

Burnham, W.L., Larson, S.P., and Cooper, H.H., Jr., 1977, Distribution of injected wastewater in the saline lava aquifer, Wailuku-Kahului Wastewater Treatment Facility, Kahului, Maui, Hawaii: U.S. Geological Survey Open-File Report 77-469, 58 p.

Cao,Y., Griffith, J.F., and Weisberg, S.B., 2009, Evaluation of optical brightener photodecay characteristics for detection of human fecal contamination: Water Research, v. 43, p. 2273-2279.

Carol, R., 2004, Maui battling seaweed invasion: Honolulu Advertiser, issue of January 5, 2004.

Casciotti, K.L., Trull, T.W., Glover, D.M., and Davies, D., 2008, Constraints on nitrogen cycling at the subtropical North Pacific Station ALOHA from isotopic measurements of nitrate and particulate nitrogen: Deep-Sea Research II, v. 55 , p. $1661-1672$.

Chen, W., Westerhoff, P., Leenheer, J.A., and Booksh, K., 2003, Fluorescence excitation-emission matrix regional integration to quantify spectra for dissolved organic matter: Environmental Science and Technology, v. 37, no. 24, p. 5701-5710.
Childress, C.J., Foreman, W.T., Connor, B.F., and Maloney, T.J., 1999, New reporting procedures based on long-term method detection levels and some considerations for interpretations of water-quality data provided by the U. S. Geological Survey National Water Quality Laboratory: U.S. Geological Survey Open-File Report 99-193, 19 p.

County of Maui, 1983, Lahaina wastewater treatment plant expansion-Final Environmental Impact Statement: State of Hawaii Office of Environmental Quality Control, 139 p.

County of Maui, 2004, Kihei wastewater reclamation facility underground injection control (UIC) injection well status report no. 19: County of Maui Wastewater Reclamation Division, $28 \mathrm{p}$.

Dailer, M., Smith, C.M., Smith, J.E., and Brown, D., 2008, Examining $\delta^{15} \mathrm{~N}$ values of intertidal macroalgae on Maui to identify locations and potential sources of nutrient enrichment, in Integrated Ecosystem Management, Maui (project summary, Celia Smith, Principal Investigator): Honolulu, University of Hawaii, [http:/www.hawaii.edu/ssri/hcri/files/ research/pdf/Smith-FY07-HCRI-NOAA-Report1.doc, last accessed November 4, 2009].

Dollar, S., 1991, An assessment of nonpoint pollution of the marine environment off Kaanapali, Maui, Hawaii: Honolulu, University of Hawaii, Final Report, 35 p.

Dollar, S., 1996, Algal blooms off West Maui_-assessing causal linkages between land and the coastal ocean, in West Maui Watershed Management Project, 1996, Algal blooms - progress report on scientific research: Maui, Hawaii, West Maui Algal Bloom Task Force, 64 p.

Dollar, S., and Andrews, C., 1997, Algal blooms off West Maui-assessing causal linkages between land and the coastal ocean: Honolulu, University of Hawaii, Final Report, $40 \mathrm{p}$.

Dollar, S.J., and Atkinson, M.J., 1992, Effects of nutrient subsidies from groundwater to nearshore marine ecosystems off the island of Hawaii: Estuarine, Coastal and Shelf Science, v. 35 , p. $409-424$.

Dollar, S., Atkinson, M., Atkinson, S., 1999, Investigation on the relation between cesspool nutrients and abundance of Hypnea musciformis in West Maui, Hawaii: Report for State of Hawaii Department of Health, Honolulu, Hawaii.

Drewes, J.E., Heberer, T., Rauch, T., and Reddersen, K., 2003, Fate of pharmaceuticals during ground water recharge: Groundwater Monitoring and Remediation, v. 23, no. 3, p. 64-72. 
Engott, J.A., and Vana, T.T., 2007, Effects of agricultural landuse changes and rainfall on groundwater recharge in central and west Maui, Hawaii, 1926-2004: U.S. Geological Survey Scientific Investigations Report 2007-5103, 56 p. [http:// pubs.usgs.gov/sir/2007/5103/, last accessed November 4, 2009].

Ferrall, C.C., Jr., 1976, Subsurface geology of Waikiki, Moiliili and Kakaako with engineering application: Honolulu, University of Hawaii, M.S. thesis, 175 p.

Fishman, M.J., ed., 1993, Methods of analysis by the U.S. Geological Survey National Water Quality Laboratorydetermination of inorganic and organic constituents in water and fluvial sediments: U.S. Geological Survey Open-File Report 93-125, 217 p.

Focazio, M.J., Kolpin, D.W., Barnes, K.K., Furlong, E.T., Meyer, M.T., Zaugg, S.D., Barber, L.B., and Thurman, E.M., 2008, A national reconnaissance for pharmaceuticals and other organic wastewater contaminants in the United States - II. Untreated drinking water sources: Science of the Total Environment, v. 402, p. 201-216.

Fujioka, R.S., and Byappanahalli, M.D., eds., 2003, Proceedings and report, Tropical Water Quality Indicator Workshop, Honolulu, Hawaii, March 1-2, 2001: Honolulu, University of Hawaii, Water Resources Research Center Special Report SR-2004-01, 60 p. and appendixes [http://www.wrrc. hawaii.edu/tropindworkshop.html, last accessed November 4, 2009].

Furlong, E.T., Werner, S.L., Anderson, B.D., and Cahill, J.D., 2008, Methods of analysis by the U.S. Geological Survey National Water Quality Laboratory - determination of human-health pharmaceuticals in filtered water by chemically modified styrene-divinylbenzene resin-based solidphase extraction and high-performance liquid chromatography/mass spectrometry: U.S. Geological Survey Techniques and Methods, book 5, chap. B5, 56 p.

Gingerich, S.B., 2008, Ground-water availability in the Wailuku area, Maui, Hawai'i: U.S. Geological Survey Scientific Investigations Report 2008-5236, 95 p, [http://pubs.usgs. gov/sir/2008/5236/, last accessed November 4, 2009].

Glassmeyer, S.T., Furlong, E.T., Kolpin, D.W., Cahill, J.D., Zaugg, S.D., Werner, S.L., Meyer, M.T., and Kryak, D.D., 2005, Transport of chemical and microbial compounds from known wastewater discharges - potential for use as indicators of human fecal contamination: Environmental Science and Technology, v. 39, no. 14, p. 5157-5169.

Grossman, E.E., and Fletcher, C.H., 1998, Sea level higher than present 3500 years ago on the Northern Main Hawaiian Islands: Geology, v. 26, no. 4, p. 363-366.
Hagedorn, C., Saluta, M., Hassall, A., and Dickerson, J., 2005, Fluorometric detection of optical brighteners as an indicator of human sources of water pollution-development as a source tracking methodology: Environmental Detection News (Virginia Tech University), v. 2, nos. 3-4, p. 1-13.

Halley, R.B., and Yates, K.K., 2008, Light, calcification, and carbonate sediment production on the Moloka i reef flat, chap. 14 of Field, M.E., Cochran, S.A., Logan, J.B., and Storlazzi C.D., eds., The coral reef of south Moloka i, Hawai $\mathrm{i}$ - portrait of a sediment-threatened fringing reef: U.S. Geological Survey Scientific Investigations Report 2007-5101, p. 117-120 [http://pubs.usgs.gov/ sir/2007/5101/sir2007-5101_chapter14.pdf, last accessed November 4, 2009].

Hannon, J.E., and Böhlke, J.K., 2008, Determination of the $\delta(15 \mathrm{~N} / 14 \mathrm{~N})$ of ammonium $(\mathrm{NH} 4+)$ in water; RSIL lab code 2898, chap. C15 of Révész, K., and Coplen, T.B., eds., Methods of the Reston Stable Isotope Laboratory: U.S. Geological Survey Techniques and Methods, book 10, section C, chapter $15,30 \mathrm{p}$.

Hartel, P.G., Hagedorn, C., and others, 2007, Exposing water samples to ultraviolet light improves fluorometry for detecting human fecal contamination: Water Research, v. 41, p. 3629-3642.

Hawaii Department of Land and Natural Resources, Division of Aquatic Resources, and Hawaii Coral Reef Initiative Research Program, 2007, Status of Maui's Reefs: Fact Sheet, 2 p.

Hawaii State Department of Health, 2004, Hawaii Administrative Rules Title 11 Department of Health Chapter 54 Water Quality Standards: [http://gen.doh.hawaii.gov/sites/har/ AdmRules1/11-54.pdf, last accessed November 4, 2009].

Hawaii State Department of Health, 2006a, Clean Water Branch: [http://www.hawaii.gov/health/environmental/ water/cleanwater/about/aboutcwb.html, last accessed November 4, 2009].

Hawaii State Department of Health, 2006b, Water quality management program: [http://www.hawaii.gov/health/ environmental/env-planning/wqm/wqm.html, last accessed November 4, 2009].

Hawaii State Department of Health, 2006c, State of Hawaii water quality monitoring and assessment report: [http:// hawaii.gov/health/environmental/env-planning/wqm/wqm. html/2006_Integrated_Report/2006_Integrated_Report.pdf, last accessed November 4, 2009].

Hawaii State Office of Planning, 2008, Hawaii Statewide GIS Program, [http://hawaii.gov/dbedt/gis/, last accessed November 4, 2009]. 
Henderson, R.K., Baker, A., Murphy, K.R., Hambly, A., Stuetz, R.M., and Khan, S.J., 2009, Fluorescence as a potential monitoring tool for recycled water system-a review: Water Research, v. 43, no. 4, p. 863-881.

Herzfeld, I., Sansone, F., Colgrove, C., Ross, M., O'Brian, M., and Smith, C., 2006, Diurnal nutrient dynamics associated with a nuisance algal bloom on South Maui, Hawaii [abs.]: Eos (American Geophysical Union Transactions), v. 87(36), Ocean Science Meeting Supplement, session 05, Hawaii [http://www.agu.org/cgi-bin/sessions5?meeting=os06\&part $=\mathrm{OS} 54 \mathrm{~J}$, last accessed November 4, 2009].

Heutmaker, D.L, Peterson, F.L., and Wheatcraft, S.W., 1977, A laboratory study of waste injection into a Ghyben-Herzberg groundwater system under dynamic conditions: Honolulu, University of Hawaii, Water Resources Research Center, Technical Report No. 107, 65 p.

Hodges, M., 1996, Na Makaala volunteer coastal monitoring team final report: Prepared for Hawaii Department of Health and NOAA Hawaiian Islands Humpback Whale National Maritime Sanctuary.

Hunt, C.D., Jr., 2004, Ground-water quality and its relation to land use on Oahu, Hawaii, 2000-01: U.S. Geological Survey Water-Resources Investigations Report 03-4305, 76 p. [http://pubs.usgs.gov/wri/wri034305/, last accessed November 4, 2009].

Hunt, C.D., Jr., 2007, Ground-water nutrient flux to coastal waters and numerical simulation of wastewater injection at Kihei, Maui, Hawaii: U.S. Geological Survey Scientific Investigations Report 2006-5283, 69 p. [http://pubs.usgs. gov/sir/2006/5283/, last accessed November 4, 2009].

Hunt, C.D., Jr., 2008, Wastewater and nutrient source tracking - a reconnaissance mapping approach for beach and watershed monitoring: U.S. Geological Survey project Web page [http://hi.water.usgs.gov/studies/project_source_ tracking.html, last accessed November 4, 2009].

Hurley, T., 2001, Algae gloom spreads along Maui coast: Honolulu Advertiser, issue of June 1, 2001.

Johnson, C.D., and White, E.A., 2007, Marine geophysical investigation of selected sites in Bridgeport, Harbor, Connecticut, 2006: U. S. Geological Survey Scientific Investigations Report 2007-5119, 32 p. [http://pubs.usgs.gov/ sir/2007/5119/, last accessed November 4, 2009].

Jurgens, B.C., McMahon, P.B., Chapelle, F.H., and Eberts, S.M., 2009, An Excel ${ }^{\circledR}$ workbook for identifying redox processes in ground water: U.S. Geological Survey Open-File Report 2009-1004, 8 p.

Kendall, C., and McDonnell, J.J., eds., 1998, Isotope tracers in catchment hydrology: Amsterdam, Elsevier Science, 839 p.
Kolpin, D.W., Furlong, E.T., Meyer, M.T., Thurman, E.M., Zaugg, S.D., Barber, L.B., and Buxton, H.T., 2002, Pharmaceuticals, hormones, and other organic wastewater contaminants in U.S. streams, 1999-2000 — a national reconnaissance: Environmental Science and Technology, v. 36, no. 6, p. $1202-1211$.

Laws, E., Brown, D., and Peace, C., 2004, Coastal water quality in the Kihei and Lahaina districts of the island of Maui, Hawaiian Islands; impacts from physical habitat and groundwater seepage; implications for water quality standards: International Journal of Environment and Pollution, v. 22 , no. 5 , p. 531-546.

LeBlanc, D.R., 1984, Sewage plume in a sand and gravel aquifer, Cape Cod, Massachusetts: U.S. Geological Survey Water-Supply Paper 2218, 28 p.

Limtiaco Consulting Group, 2005, 2004 Hawaii water reuse survey and report - final: Honolulu, Prepared for Hawaii State Department of Land and Natural Resources, Commission on Water Resources Management, 100 p. and Appendixes.

Liu, C.C.K., 2007, RAM2 modeling and the determination of sustainable yields of Hawaii basal aquifers: Honolulu, University of Hawaii, Water Resources Research Center Project Report PR-2008-06, 75 p. and appendix.

Manheim, F.T., Krantz, D.E., Snyder, D.S., and Sturgis, B., 2002, Streamer resistivity surveys in Delmarva Coastal Bays: Proceedings Symposium on the Application of Geophysics to Environmental and Engineering Problems (SAGEEP), 2002, paper 13GSL5, 17 p. [http://woodshole. er.usgs.gov/staffpages/fmanheim/delmarva.htm, last accessed November 4, 2009].

McMahon, P.B., and Chapelle, F.H., 2008, Redox processes and water quality of selected principal aquifer systems: Ground Water, v. 46, no. 2, p. 259-271.

Mengis, M., Schiff, S.L., Harris, M., English, M.C., and others, 1999, Multiple geochemical and isotopic approaches for assessing ground water $\mathrm{NO}_{3}$ - elimination in a riparian zone: Ground Water, v. 37, no. 3, p. 448-457.

Miller, D.N., and Smith, R.L., 2009, Microbial characterization of nitrification in a shallow, nitrogen contaminated aquifer, Cape Cod, Massachusetts and detection of a novel cluster associated with nitrifying Betaproteobacteria: Journal of Contaminant Hydrology, v. 103, p. 182-193.

Nyquist, J.E., Freyer, P.A., and Toran, L., 2008, Stream bottom resistivity tomography to map ground water discharge: Ground Water, v. 46, no. 4, p. 561-569.

Oberdorfer, J.A., and Peterson, F.L, 1982, Wastewater injection well problems, processes, and standards: Honolulu, University of Hawaii, Water Resources Research Center, Technical Report No. 146, 131 p. 
Oki, D.S., 2005, Numerical simulation of the effects of lowpermeability valley-fill barriers and the redistribution of ground-water withdrawals in the Pearl Harbor area, Oahu, Hawaii: U.S. Geological Survey Scientific Investigations Report 2005-5253, 111 p.

Palmer, H.S., 1927, The geology of the Honolulu artesian system: Supplement to the Report of the Honolulu Sewer and Water Commission, 68 p.

Parabicoli, S., 2007, Maui's growing water reuse experience: Proceedings of the Water Environment Federation, WEFTEC 2007, Session 91 through Session 100, p. $7546-7564$.

Patton, C.J., and Kryskalla, J.R., 2003, Methods of analysis by the U.S. Geological Survey National Water Quality Laboratory - evaluation of alkaline persulfate digestion as an alternative to Kjeldahl digestion for determination of total and dissolved nitrogen and phosphorus in water: U.S. Geological Survey Water-Resources Investigations Report 03-4174, $33 \mathrm{p}$.

Peterson, F.L., Williams, J.A., Wheatcraft, S.W., 1978, Waste injection into a two-phase flow field; sand-box and HeleShaw models study: Ground Water, v. 16, no. 6, p. 410-416.

Petty, S., and Peterson, F.L., 1979, Hawaiian waste injection practices and problems: Honolulu, University of Hawaii, Water Resources Research Center, Technical Report No. 123, 104 p.

Phillips, P., and Chalmers, A., 2009, Wastewater effluent, combined sewer overflows, and other sources of organic compounds to Lake Champlain: Journal of the American Water Resources Association, v. 45, no. 1, p. 45-57.

Révész, K., and Casciotti, K., 2007a, Determination of the $\delta\left({ }^{15} \mathrm{~N} / 14 \mathrm{~N}\right)$ of nitrate in water: RSIL Lab Code 2899, chap. C16 of Révész, K., and Coplen, T.B., eds., Methods of the Reston Stable Isotope Laboratory: Reston, Virginia, U.S. Geological Survey, Techniques and Methods, book 10, section $\mathrm{C}$, chap. 16, $24 \mathrm{p}$.

Révész, .K., and Casciotti, K., 2007b, Determination of the $\delta\left({ }^{15} \mathrm{~N} / 14 \mathrm{~N}\right)$ and $\delta(18 \mathrm{O} / 16 \mathrm{O})$ of nitrate in water: RSIL Lab Code 2900, chap. C17 of Révész, K., and Coplen, T.B., eds., Methods of the Reston Stable Isotope Laboratory: Reston, Virginia, U.S. Geological Survey, Techniques and Methods, book 10, section C, chap. 17, 24 p.

Révész, K., and Coplen, T.B., 2008a, Determination of the $\delta(2 \mathrm{H} / 1 \mathrm{H})$ of water: RSIL lab code 1574 , chap. C1 of Révész, K., and Coplen, T.B., eds., Methods of the Reston Stable Isotope Laboratory: U.S. Geological Survey Techniques and Methods book 10, section C, chap. 1, 27 p.
Révész, K., and Coplen, T.B., 2008b, Determination of the $\delta(18 \mathrm{O} / 16 \mathrm{O})$ of water: RSIL lab code 489 , chap. C2 of Révész, K., and Coplen, T.B., eds., Methods of the Reston Stable Isotope Laboratory: U.S. Geological Survey Techniques and Methods, book 10, section C, chap. 2, 28 p.

Révész, K., and Qi, H., 2006, Determination of the $\delta\left({ }^{15} \mathrm{~N} / 14 \mathrm{~N}\right)$ of total nitrogen in solids: RSIL Lab Code 2893, chap. C11 of Révész, K., and Coplen, T.B., eds., Methods of the Reston Stable Isotope Laboratory: Reston, Virginia, U.S. Geological Survey, Techniques and Methods, book 10, section C, chap. 11, 31 p.

Scholl, M.A., Ingebritsen, S.E., Janik, C.J., and Kauahikaua, J.P., 1995, An isotope hydrology study of the Kilauea Volcano area, Hawaii: U.S. Geological Survey Water Resources Investigations Report 95-4213, 44 p.

Scholl, M.A., Gingerich, S.B., and Tribble, G.W., 2002, The influence of microclimates and fog on stable isotope signatures used in interpretation of regional hydrology-East Maui, Hawaii: Journal of Hydrology, v. 264, p. 170-184.

Schwartz, M., ed., 2006, Encyclopedia of coastal science: Berlin, Springer, $1211 \mathrm{p}$.

Seitzinger, S.P., Sanders, R.W, and Styles, R., 2002, Bioavailability of DON from natural and anthropogenic sources to estuarine plankton: Limnology and Oceanography, v. 47, no. 2, p. 353-366.

Shade, P.J., 1996, Water budget for the Lahaina District, Island of Maui, Hawaii: U.S. Geological Survey Water-Resources Investigations Report 96-4238, 27 p.

Sherrod, D.R., Sinton, J.M., Watkins, S.E., and Brunt, K.M., 2007, Geologic map of the State of Hawaii: U.S. Geological Survey Open-File Report 2007-1089, 83 p., 8 plates, scales $1: 100,000$ and 1:250,000, with GIS database.

Smith, C.M., and Smith, J.E., 2007, Algal blooms in north Kihei; an assessment of patterns \& processes relating nutrient dynamics to algal abundance: University of Hawaii, Report to City and County of Maui, 65 p.

Smith, J.E., Runcie, J.W., and Smith, C.M., 2005, Characterization of a large-scale ephemeral bloom of the green alga Cladophora sericea on the coral reefs of West Maui, Hawaii: Marine Ecology Progress Series, v. 302, p. 77-91.

Smith, R.L., and Duff, J.H., 1988, Denitrification in a sand and gravel aquifer: Applied and Environmental Microbiology, v. 54, no. 5, p. 1071-1078.

Smith, R.L., Howes, B.L., and Duff, J.H., 1991, Denitrification in nitrate-contaminated ground water; occurrence in steep vertical geochemical gradients: Geochimica et Cosmochimica Acta, v. 55, no. 7, p. 1815-1825. 
Smith, R.L., Miller, D.N., Brooks, M.H., Widdowson, M.A., and Killingstad, M.W., 2001, In situ stimulation of groundwater denitrification with formate to remediate nitrate contamination: Environmental Science and Technology, v. 35, no. 1, p. 196-203.

Soicher, A.J., and Peterson, F.L., 1997, Terrestrial nutrient and sediment fluxes to the coastal waters of west Maui, Hawaii: Pacific Science, v. 51, no. 3, p. 221-232.

Souza, W.R., 1981, Ground-water status report, Lahaina District, Maui, Hawaii, 1980: U.S. Geological Survey OpenFile Report 81-549, 2 map sheets.

Stearns, H.T., and Macdonald, G.A., 1942, Geology and ground-water resources of the island of Maui, Hawaii: Hawaii (Territory) Division of Hydrography Bulletin 7, 334 p.

Stewart, R.H., 2005, Introduction to physical oceanography: Texas A\&M University, online textbook [http://oceanworld. tamu.edu/home/course_book.htm, last accessed November 4, 2009].

Street, J.H., Knee, K.L., Grossman, E.E., and Paytan, A., 2008, Submarine groundwater discharge and nutrient addition to the coastal zone and coral reefs of leeward Hawaii: Marine Chemistry, v. 109, p. 355-376.

Takasaki, K.J., 1972, Preliminary report on the water resources of Central Maui: State of Hawaii, Department of Land and Natural Resources, Division of Water and Land Development, Circular C62, 59 p. plus appendixes.

Taylor, L.A., Eakins, B.W., Carignan, K.S., Warnken, R.R., Sazonova, T., and Schoolcraft, D.C., 2008, Digital elevation model of Lahaina, Hawaii-procedures, data sources and analysis: Boulder, Colorado, National Geophysical Data Center, NOAA Technical Memorandum NESDIS NGDC$10,19 \mathrm{p}$.

Tetra Tech, Inc., 1993, Preliminary assessment of possible anthropogenic nutrient sources in the Lahaina District of Maui: Prepared for U.S. Environmental Protection Agency Region 9, 115 p. plus appendixes.

Tetra Tech, Inc., 1994, Effluent fate study, Lahaina wastewater reclamation facility, Maui, Hawaii: Prepared for U.S. Environmental Protection Agency Region 9, 73 p. plus appendixes.

Tribble, G.W., Sansone, F.J., and Smith, S.V., 1990, Stoichiometric modeling of anaerobic diagenesis within a coral reef framework: Geochimica et Cosmochimica Acta, v. 54, p. 2439-2449.
Turner Designs, 2004, Aquafluor handheld fluorometer and turbidimeter; user's manual: Sunnyvale, Calif., 36 p. [http:// www.turnerdesigns.com/t2/instruments/aquafluor.html, last accessed November 4, 2009].

Turner Designs, 2007a, CDOM instrumentation and optical kit ordering information: Application note S-0022 [http:// www.turnerdesigns.com/t2/doc/appnotes/S-0022.pdf, last accessed November 4, 2009].

Turner Designs, 2007b, Optical brighteners: Application note S-0080 [http://www.turnerdesigns.com/t2/doc/ appnotes/S-0080.pdf, last accessed November 4, 2009].

U.S. Environmental Protection Agency, 2006, Beach standards, monitoring, and notification: [http://www.epa.gov/ waterscience/beaches/, last accessed November 4, 2009].

U.S. Geological Survey, variously dated, National field manual for the collection of water-quality data: U.S. Geological Survey Techniques of Water-Resources Investigations, book 9, chaps. A1-A9, [http://water.usgs.gov/owq/FieldManual/, last accessed November 4, 2009].

Wagner, R.J., Boulger, R.W., Jr., Oblinger, C.J., and Smith, B.A., 2006, Guidelines and standard procedures for continuous water-quality monitors; station operation, record computation, and data reporting: U.S. Geological Survey Techniques and Methods book 1, section D, chap. 3, 51 p. plus attachments [http://pubs.usgs.gov/tm/2006/tm1D3/, last accessed November 4, 2009].

Walter, D.A., Rea, B.A., Stollenwerk, K.G., and Savoie, J., 1996, Geochemical and hydrologic controls on phosphorus transport in a sewage-contaminated sand and gravel aquifer near Ashumet Pond, Cape Cod, Massachusetts: U.S. Geological Survey Water-Supply Paper 2463, 89 p.

West Maui Watershed Management Project, 1996, Algal blooms - progress report on scientific research: Maui, Hawaii, West Maui Watershed Algal Bloom Task Force, 64 p.

West Maui Watershed Management Advisory Committee, 1997, West Maui watershed owners manual: Maui, Hawaii, $104 \mathrm{p}$.

Wheatcraft, S.W., Peterson, F.L., and Heutmaker, D.L., 1976, Waste injection into the Hawaiian Ghyben-Herzberg aquifer - a laboratory study using a sand-packed hydraulic model: Honolulu, University of Hawaii, Water Resources Research Center, Technical Report No. 96, 69 p. plus appendixes.

Yamanaga, G., and Huxel, C.J., 1969, Preliminary report on the water resources of the Lahaina District, Maui: State of Hawaii, Department of Land and Natural Resources, Division of Water and Land Development, Circular C51, 47 p. 
Zaugg, S.D., Smith, S.G., Schroeder, M.P., Barber, L.B., and Burkhardt, M.R., 2002, Methods of analysis by the U.S. Geological Survey National Water Quality Laboratorydetermination of wastewater compounds by polystyrenedivinylbenzene solid-phase extraction and capillarycolumn gas chromatography/mass spectrometry: U.S. Geological Survey Water-Resources Investigations Report $01-4186,37 \mathrm{p}$. 


\section{Appendix A. \\ Maps and tables of Results from Wading and Kayak Instrument Surveys at Kihei and Lahaina, Hawaii}




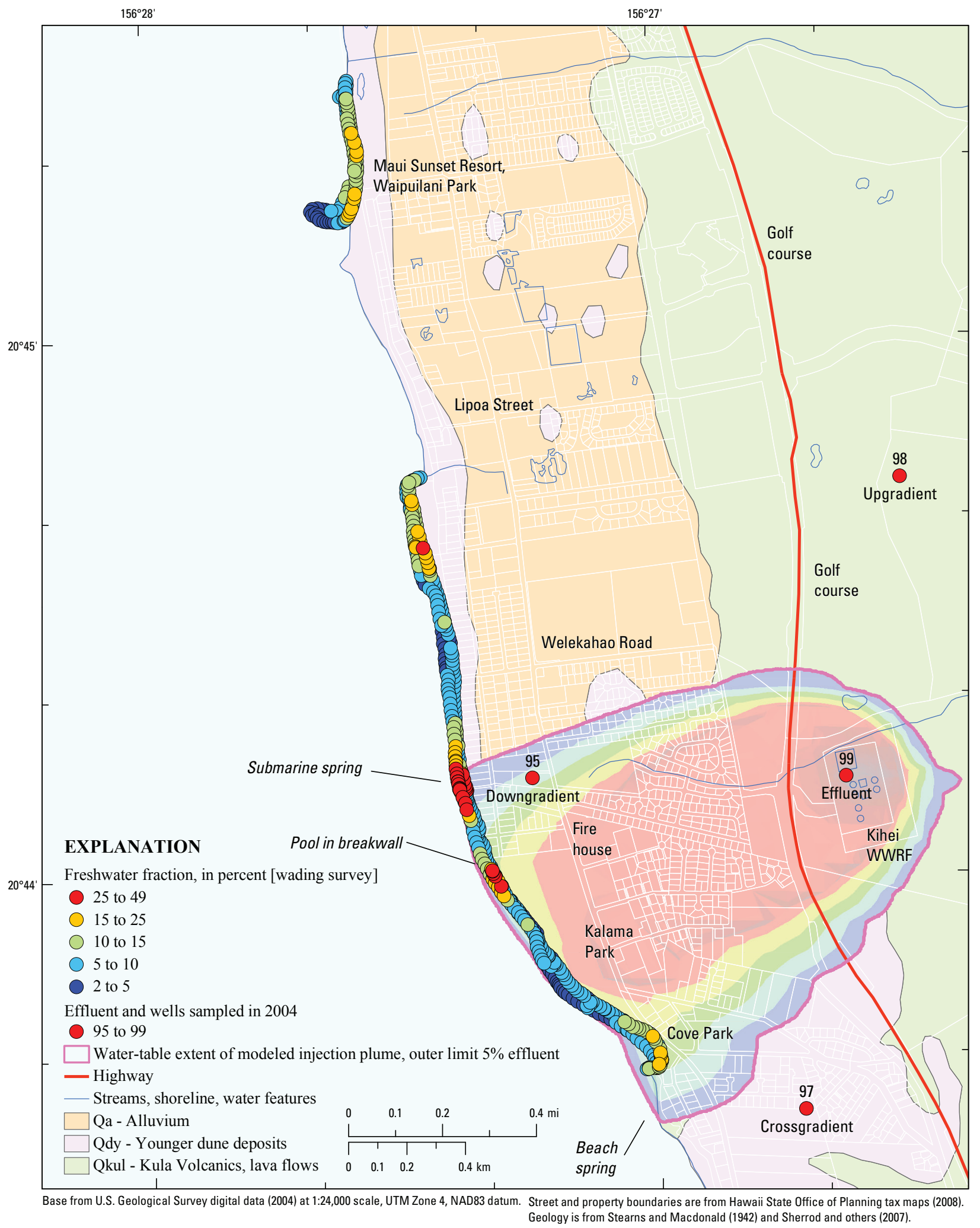

Figure A1. Freshwater fraction (computed from salinity) along wading survey at Kihei, Hawaii. 


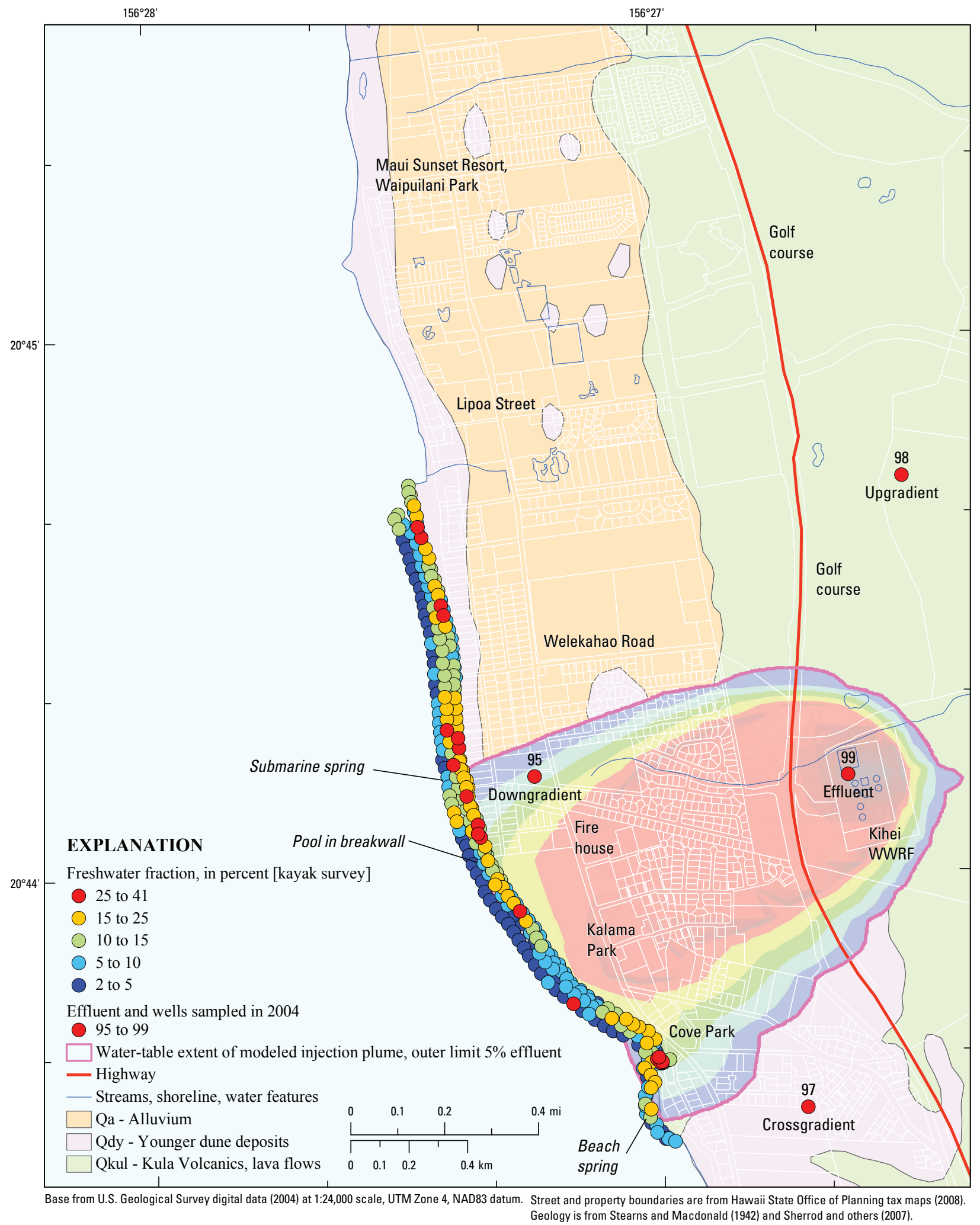

Figure A2. Freshwater fraction (computed from salinity) along kayak survey at Kihei, Hawaii. 


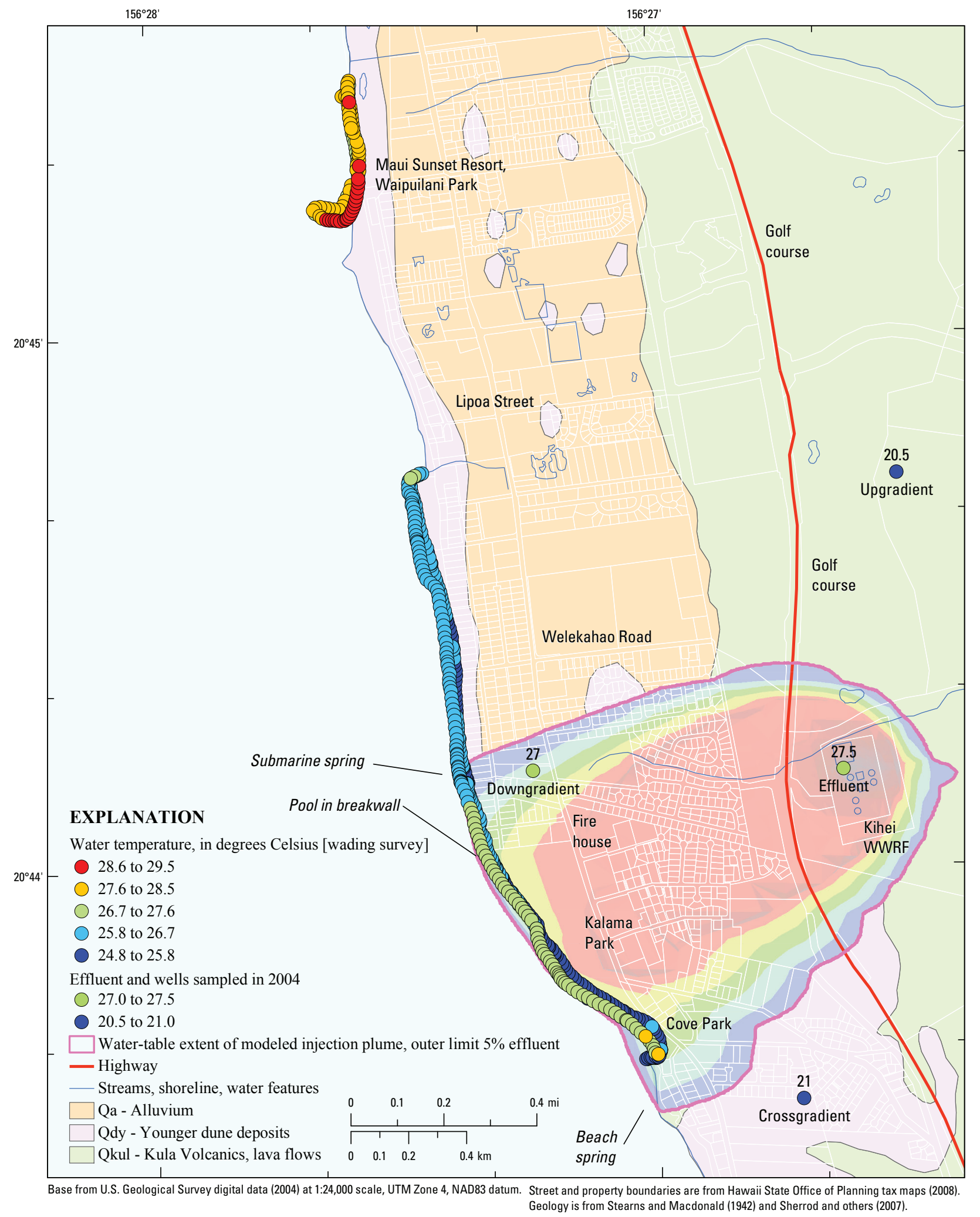

Figure A3. Water temperature along wading survey at Kihei, Hawaii. 


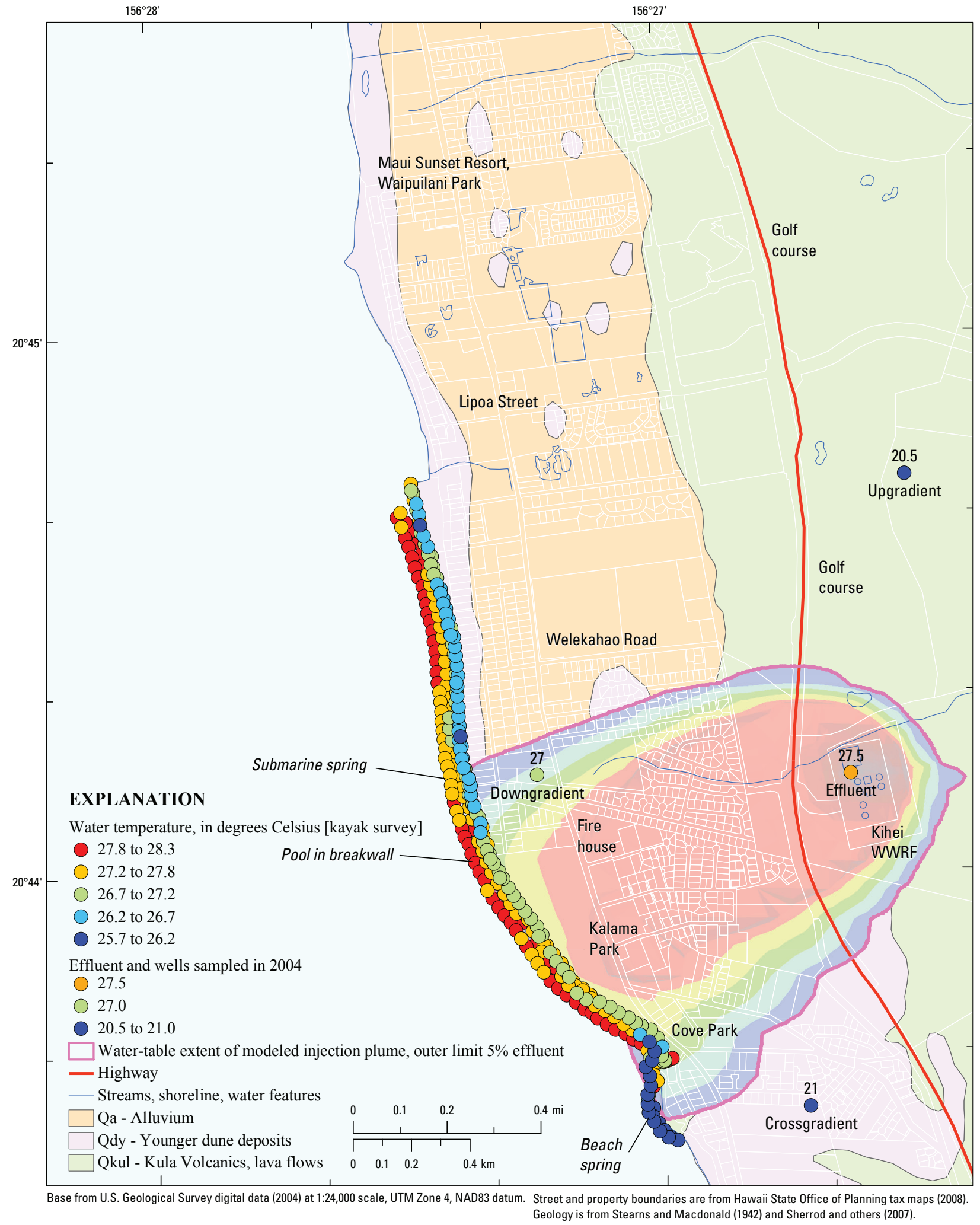

Figure A4. Water temperature along kayak survey at Kihei, Hawaii. 


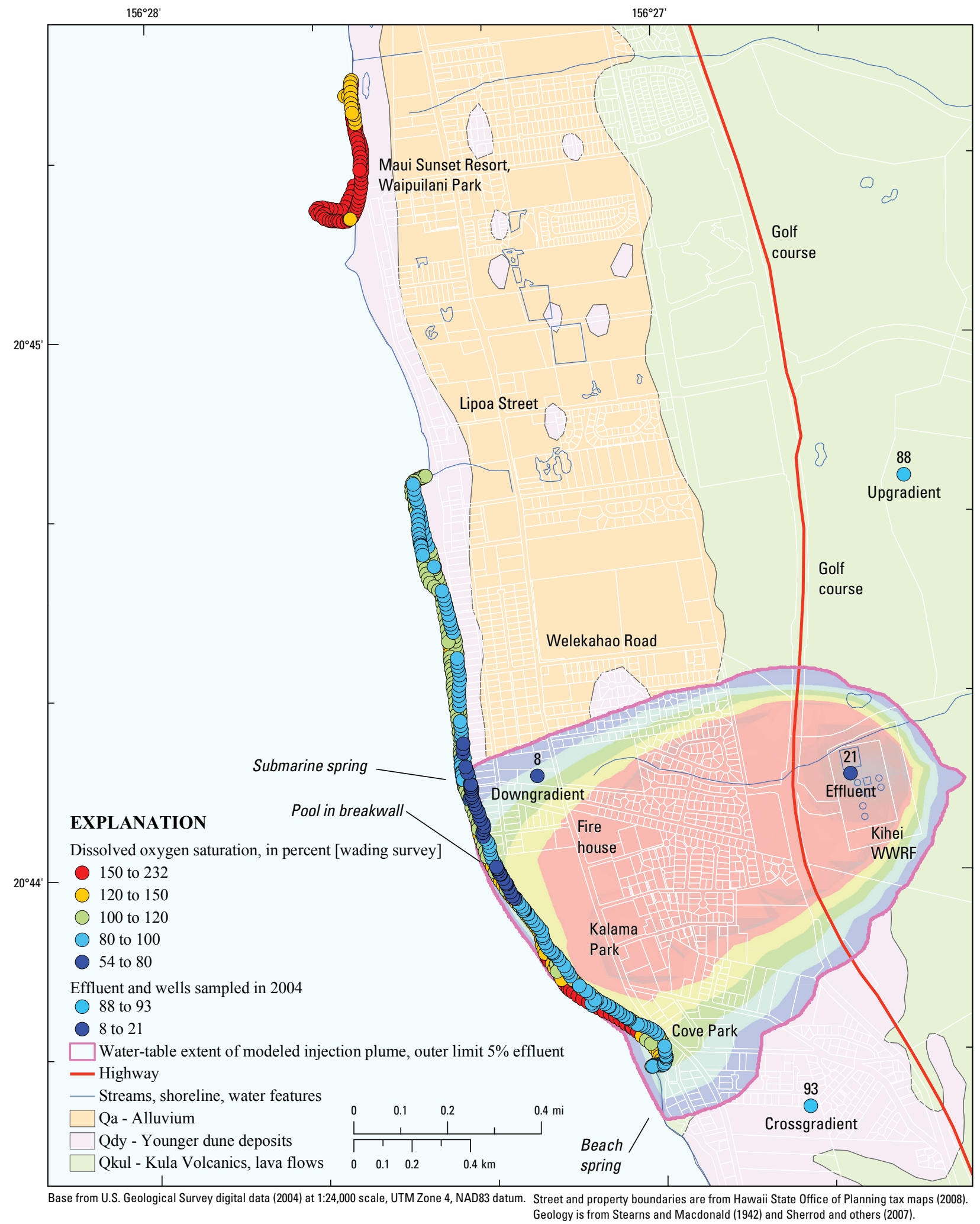

Figure A5. Dissolved oxygen saturation along wading survey at Kihei, Hawaii. 


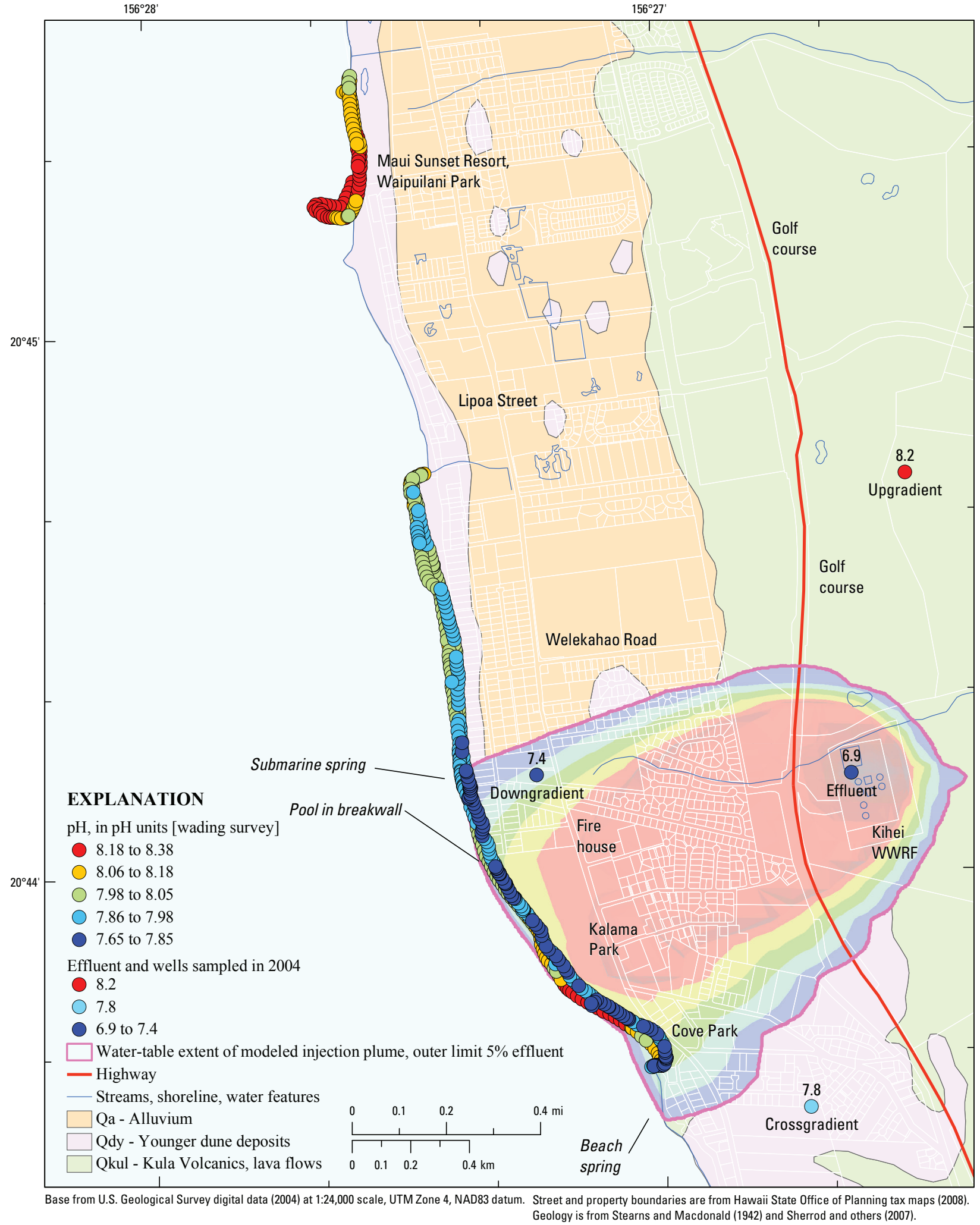

Figure A6. pH along wading survey at Kihei, Hawaii. 


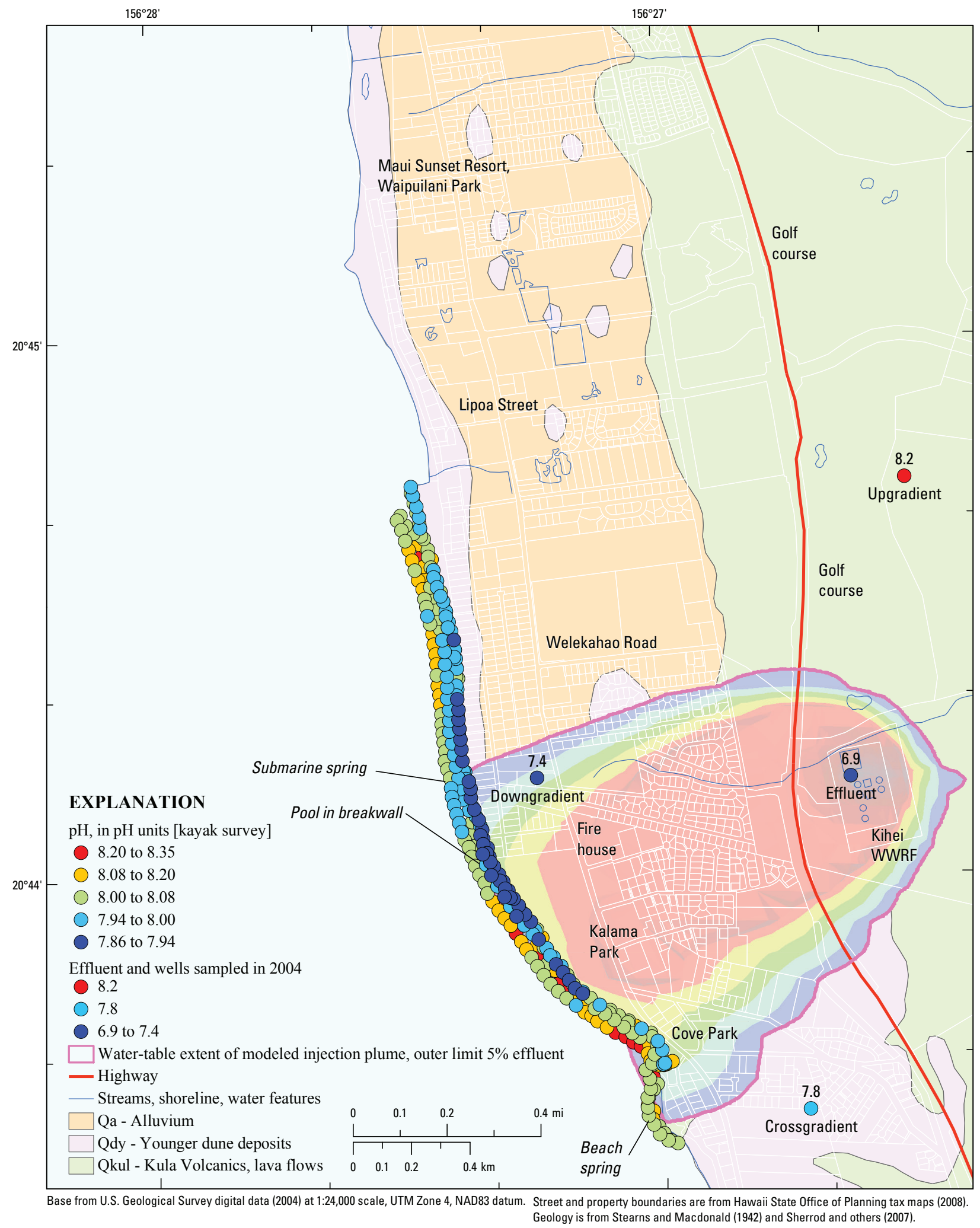

Figure A7. pH along kayak survey at Kihei, Hawaii. 


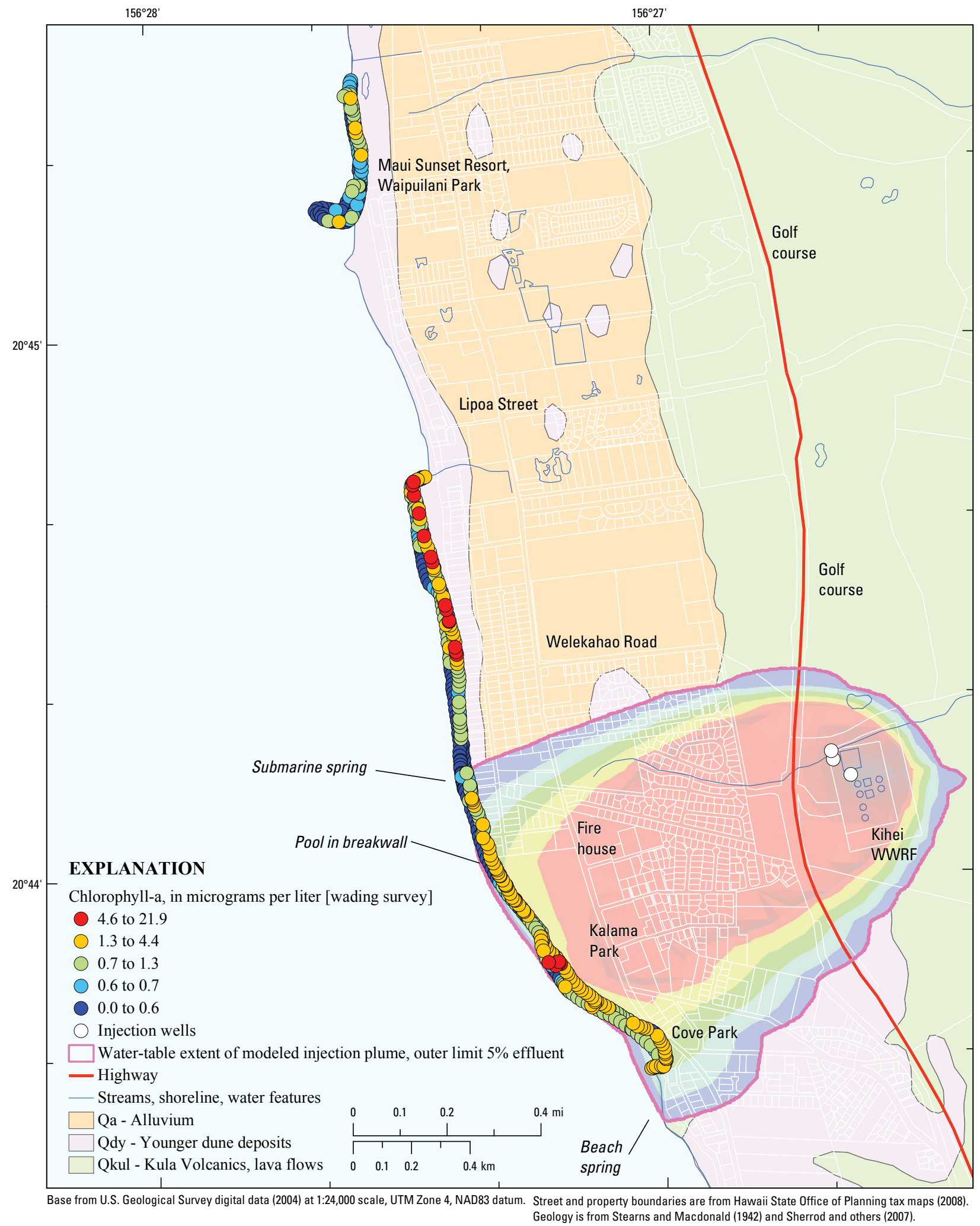

Figure A8. Chlorophyll-a concentration along wading survey at Kihei, Hawaii. 


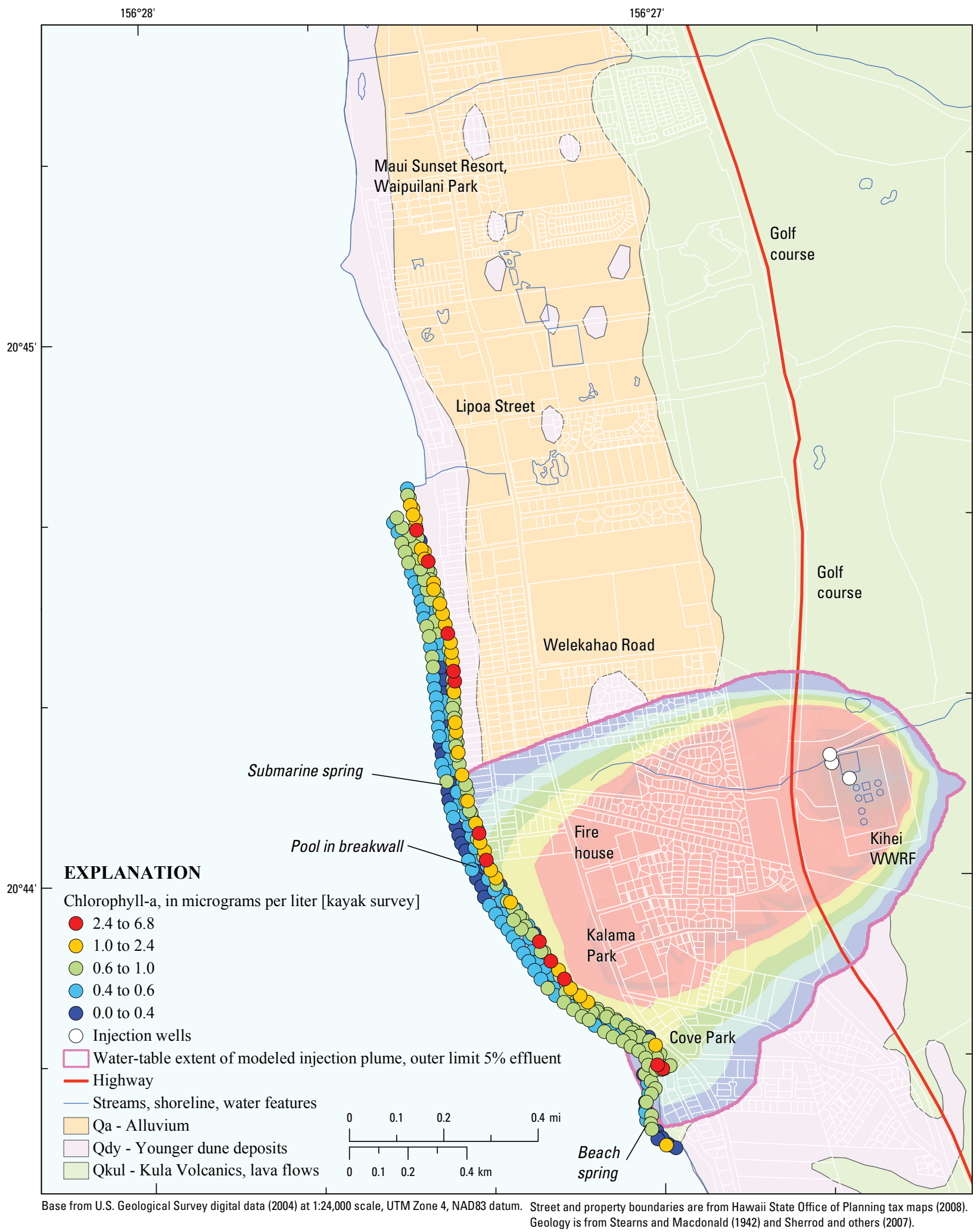

Figure A9. Chlorophyll-a concentration along kayak survey at Kihei, Hawaii. 


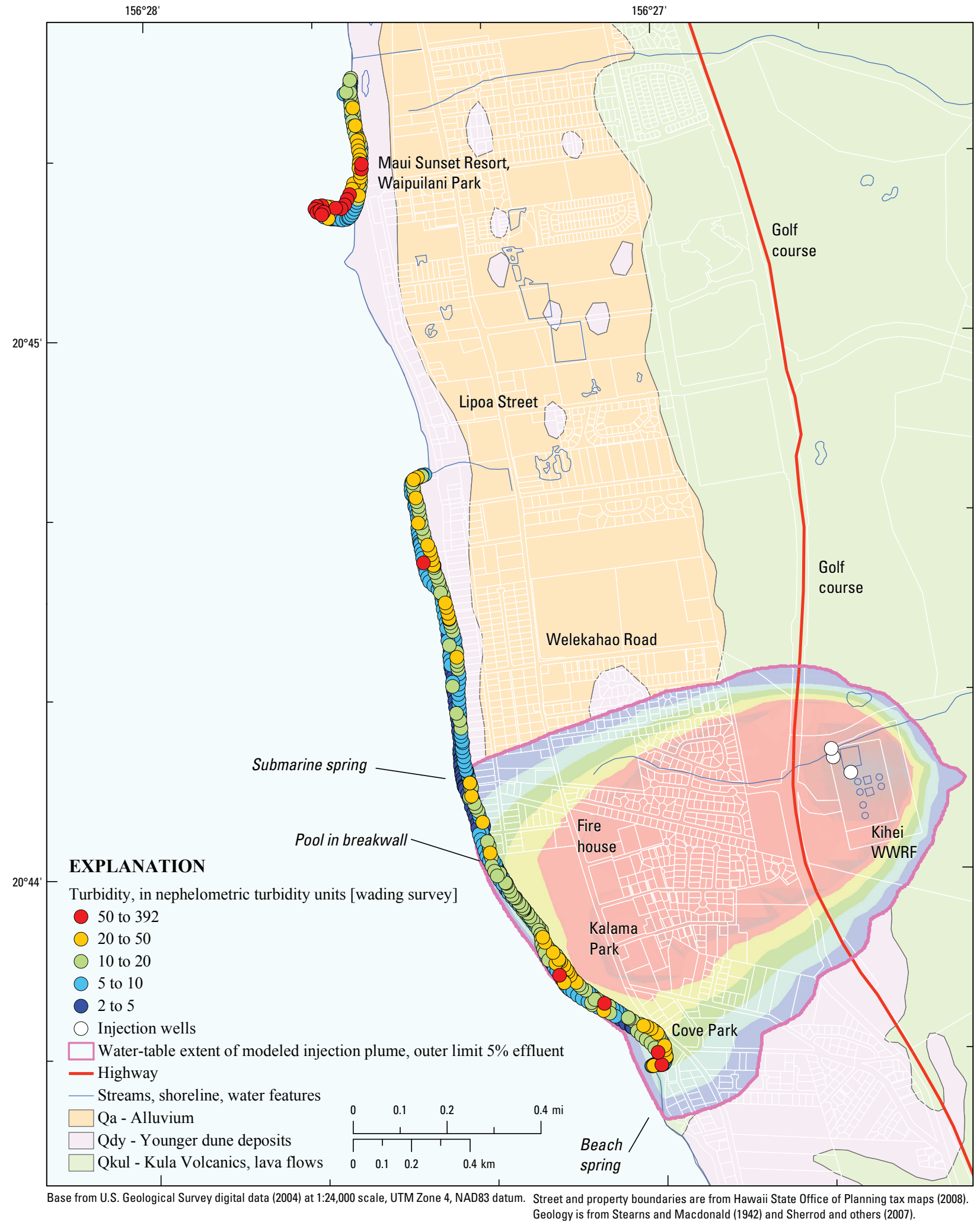

Figure A10. Turbidity along wading survey at Kihei, Hawaii. 


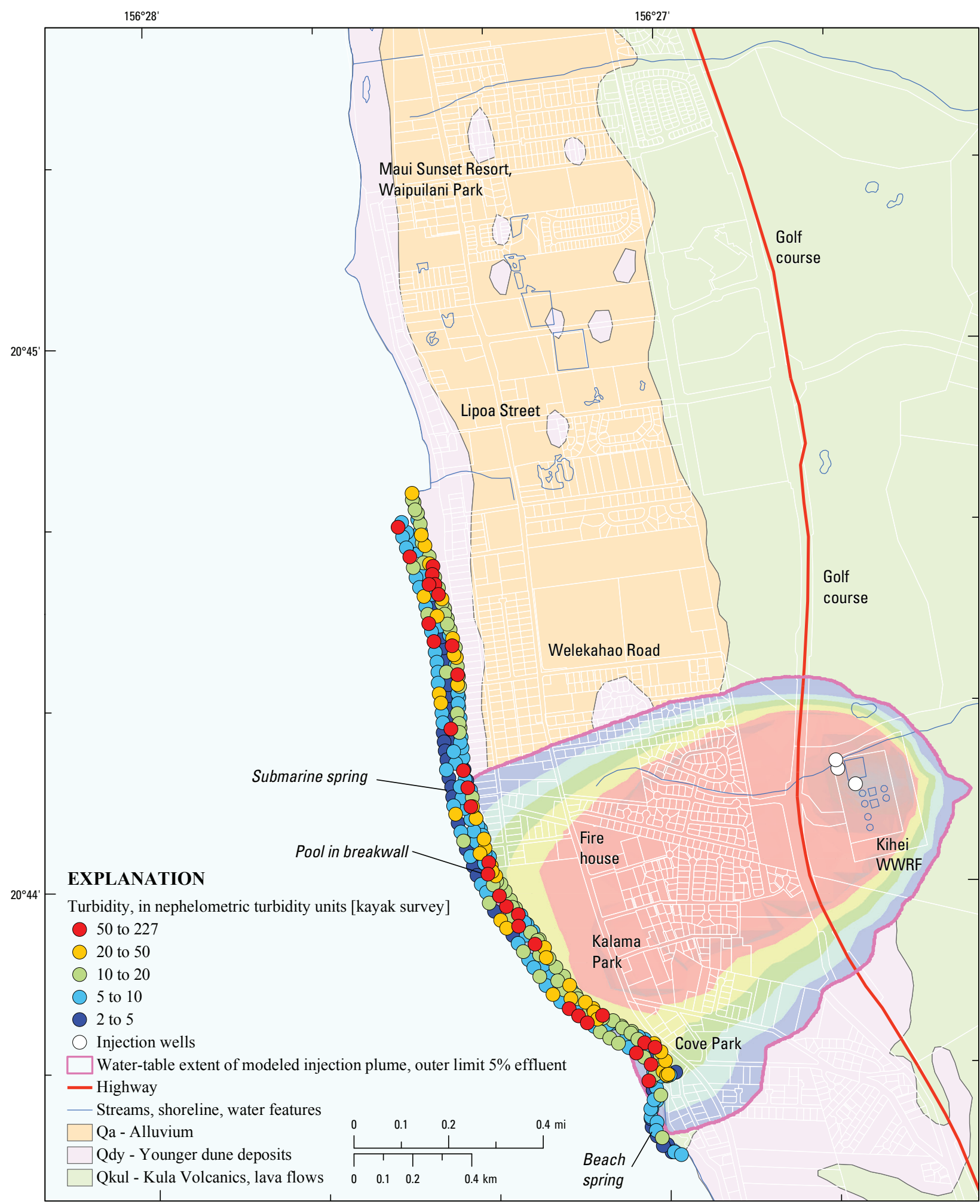

Base from U.S. Geological Survey digital data (2004) at 1:24,000 scale, UTM Zone 4, NAD83 datum. Street and property boundaries are from Hawaii State 0ffice of Planning tax maps (2008) Geology is from Stearns and Macdonald (1942) and Sherrod and others (2007).

Figure A11. Turbidity along kayak survey at Kihei, Hawaii. 


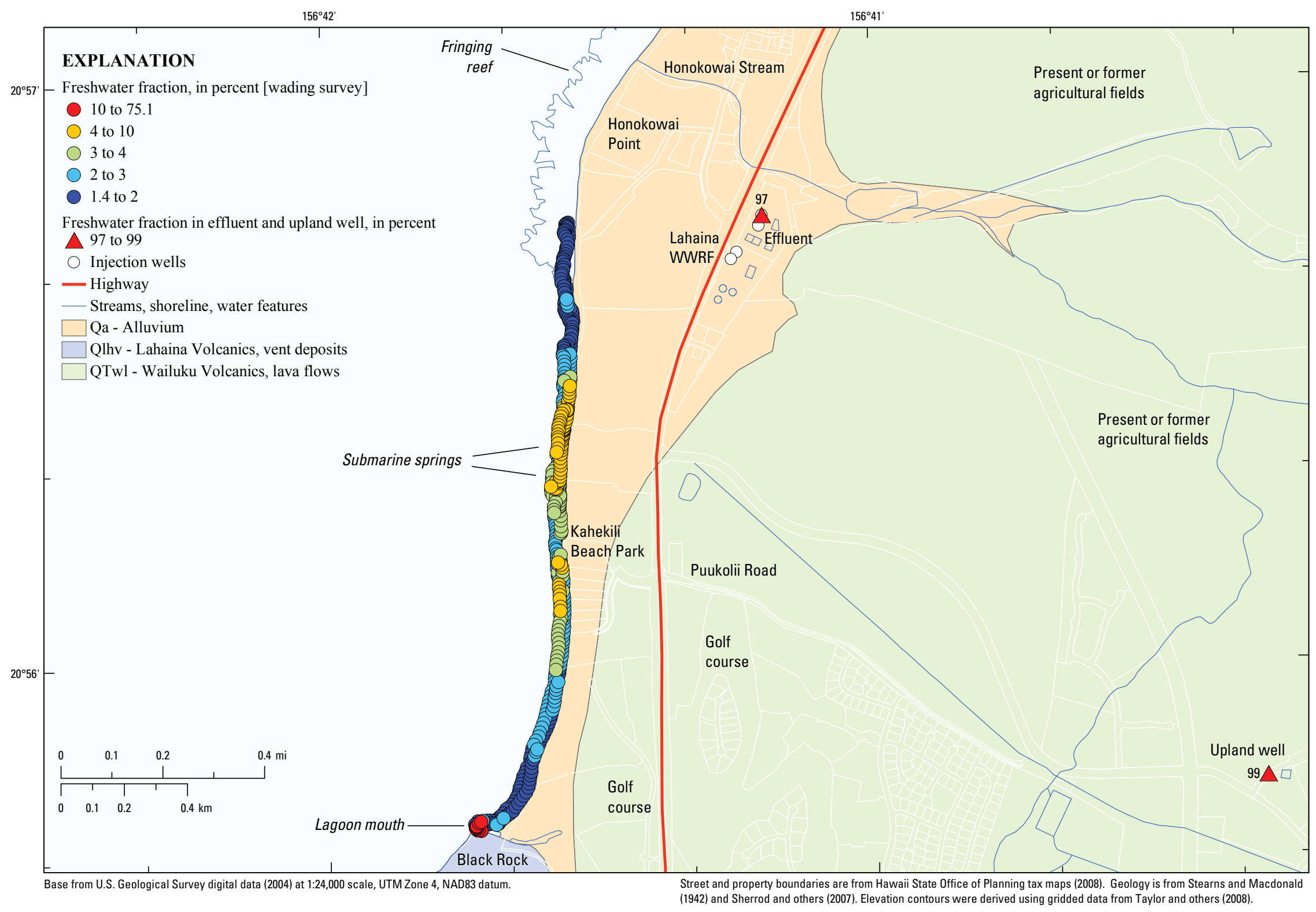

Figure A12. Freshwater fraction (computed from salinity) along wading survey at Lahaina, Hawaii. 


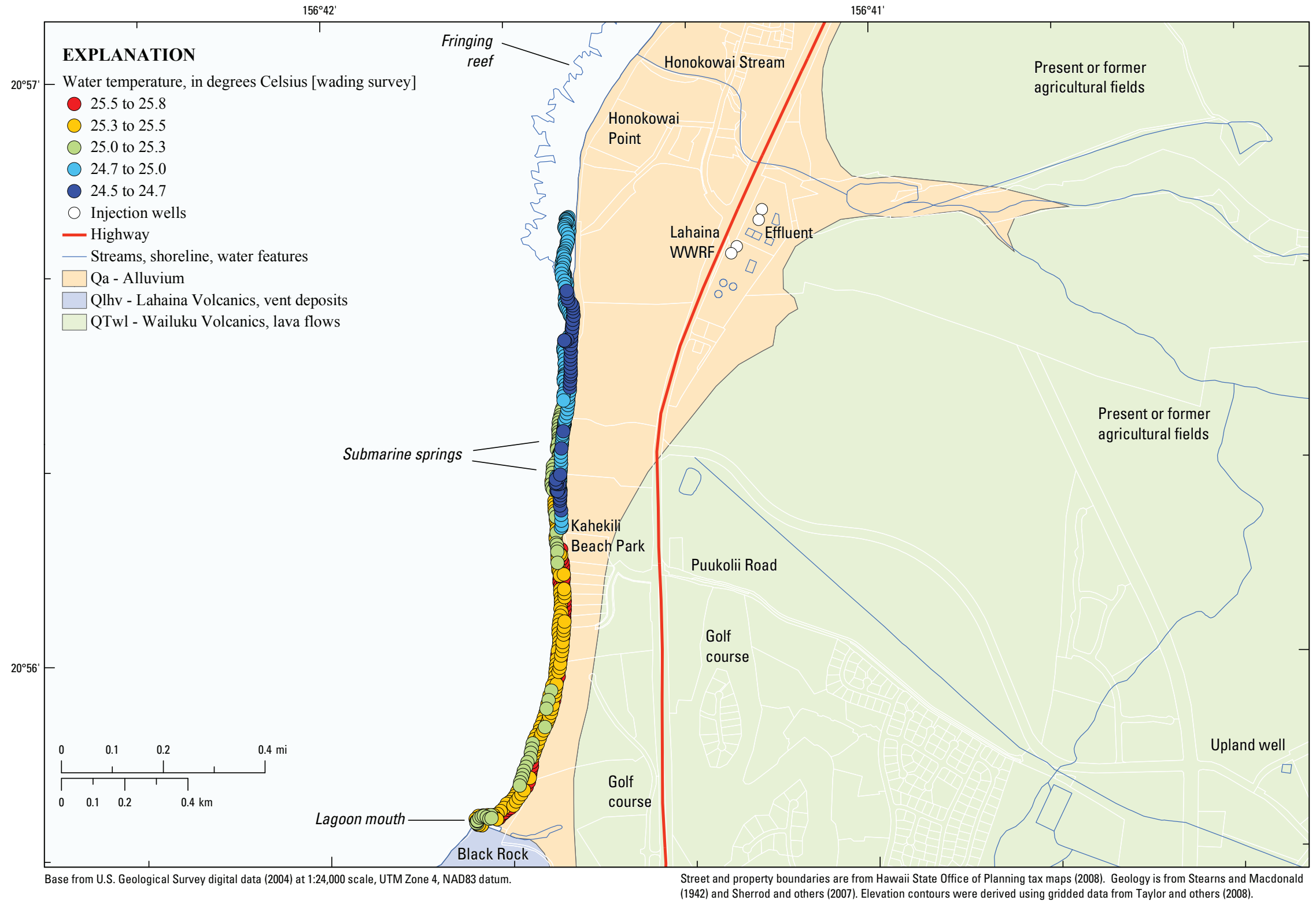

Figure A13. Water temperature along wading survey at Lahaina, Hawaii. 


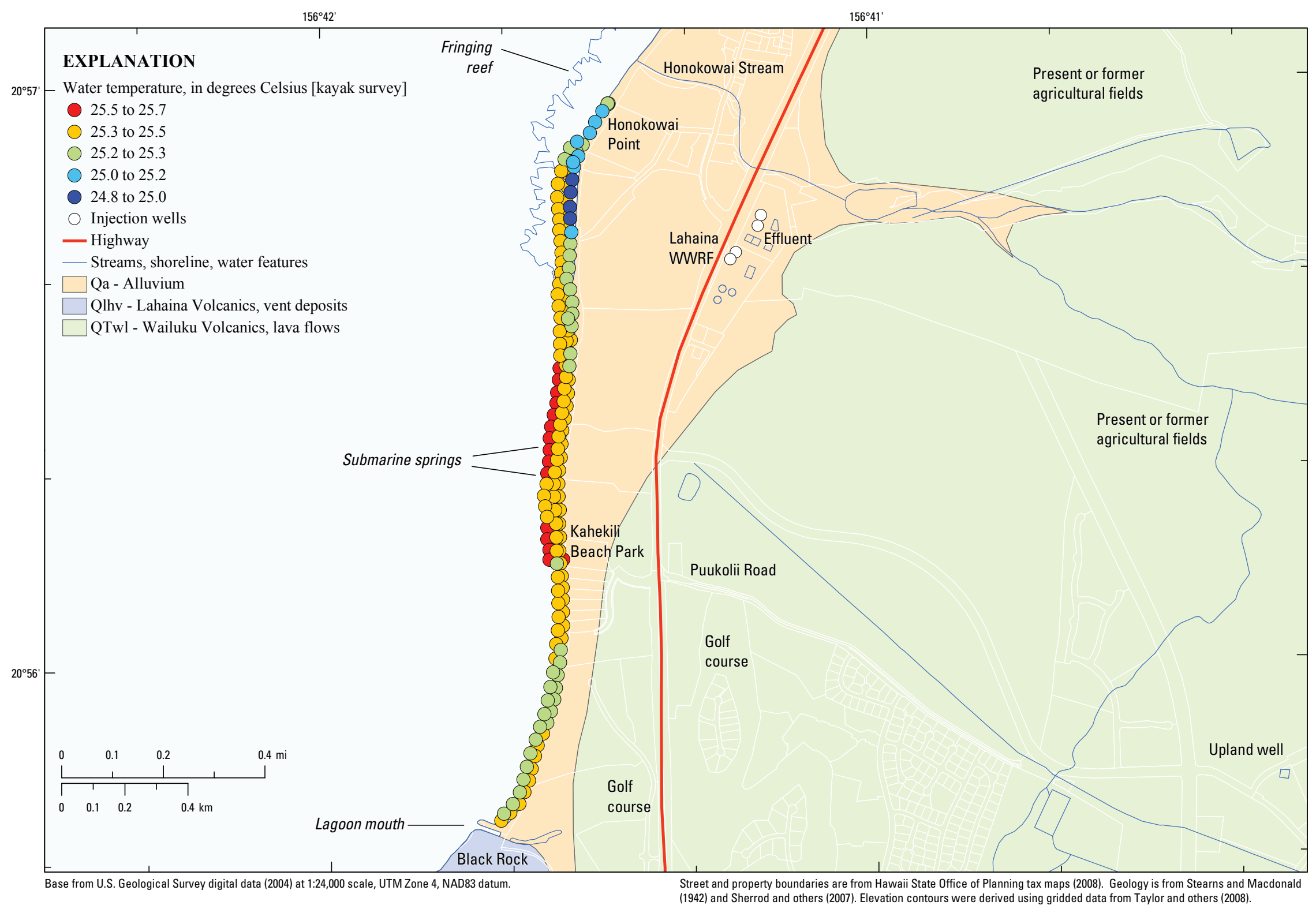

Figure A14. Water temperature along kayak survey at Lahaina, Hawaii. 
$156^{\circ} 42$

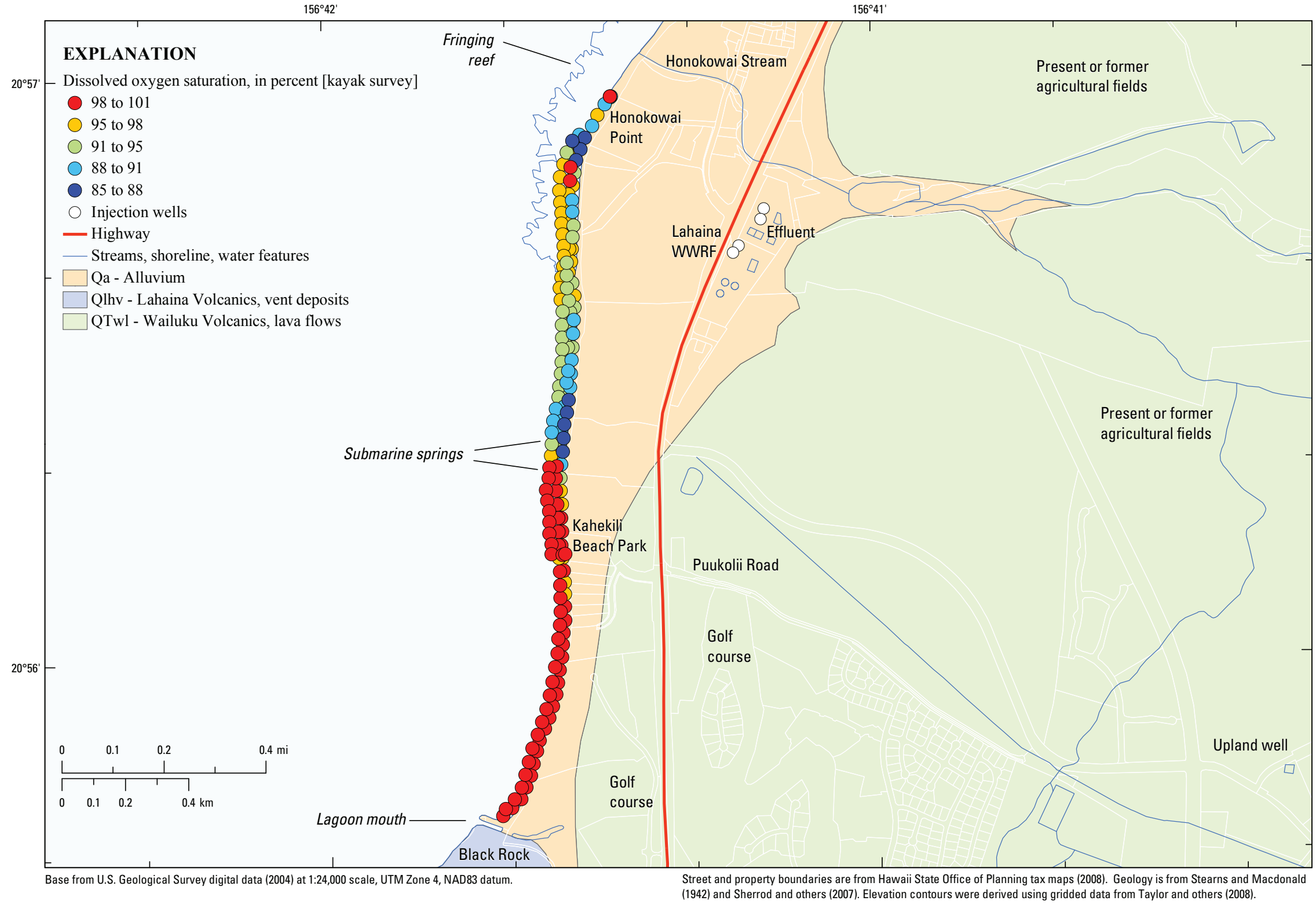

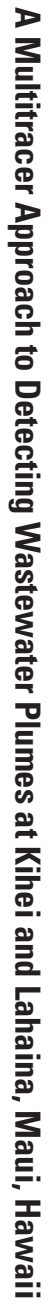

Figure A15. Dissolved oxygen saturation along kayak survey at Lahaina, Hawaii. 


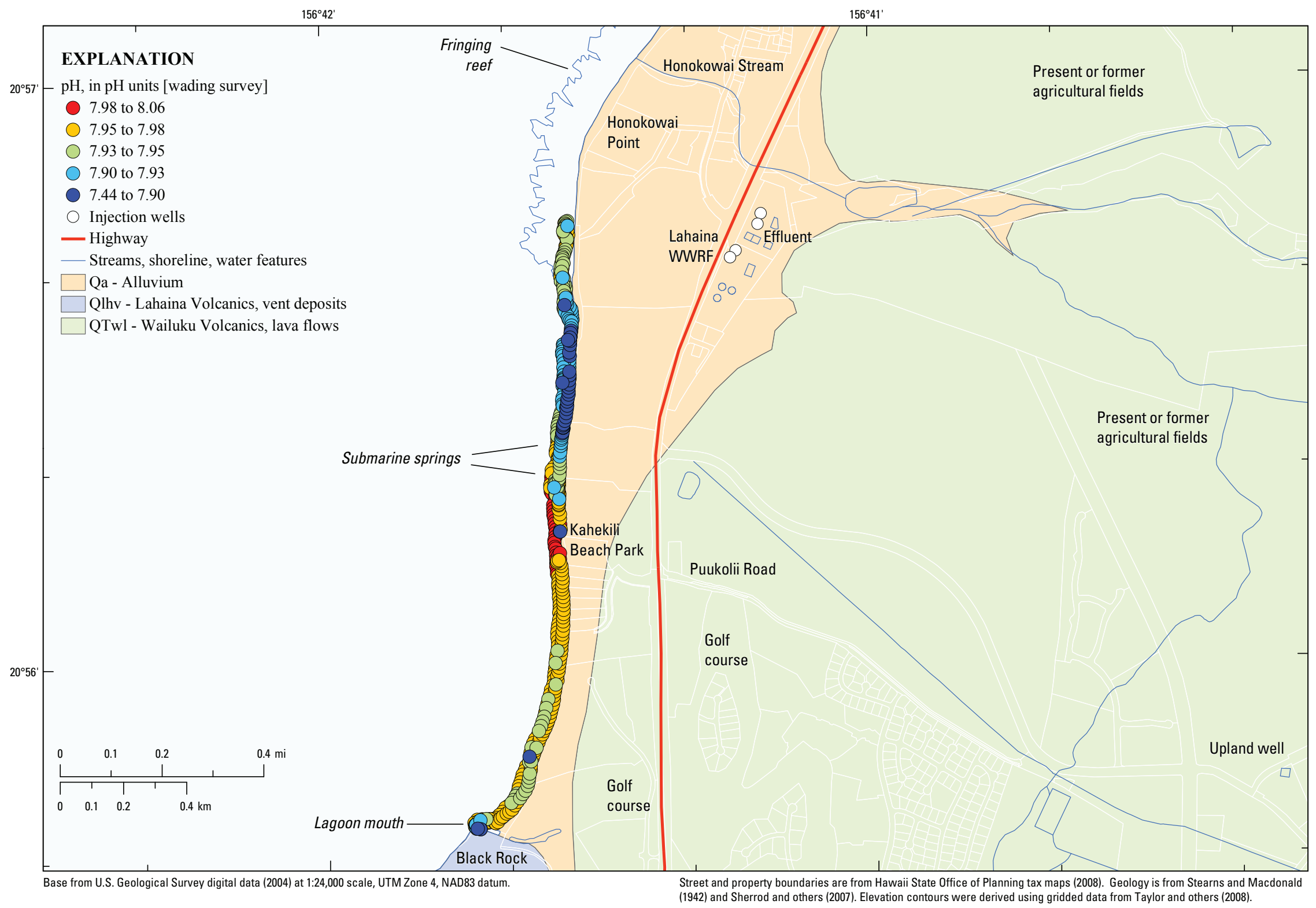

Figure A16. pH along wading survey at Lahaina, Hawaii. 
$156^{\circ} 42^{\prime}$

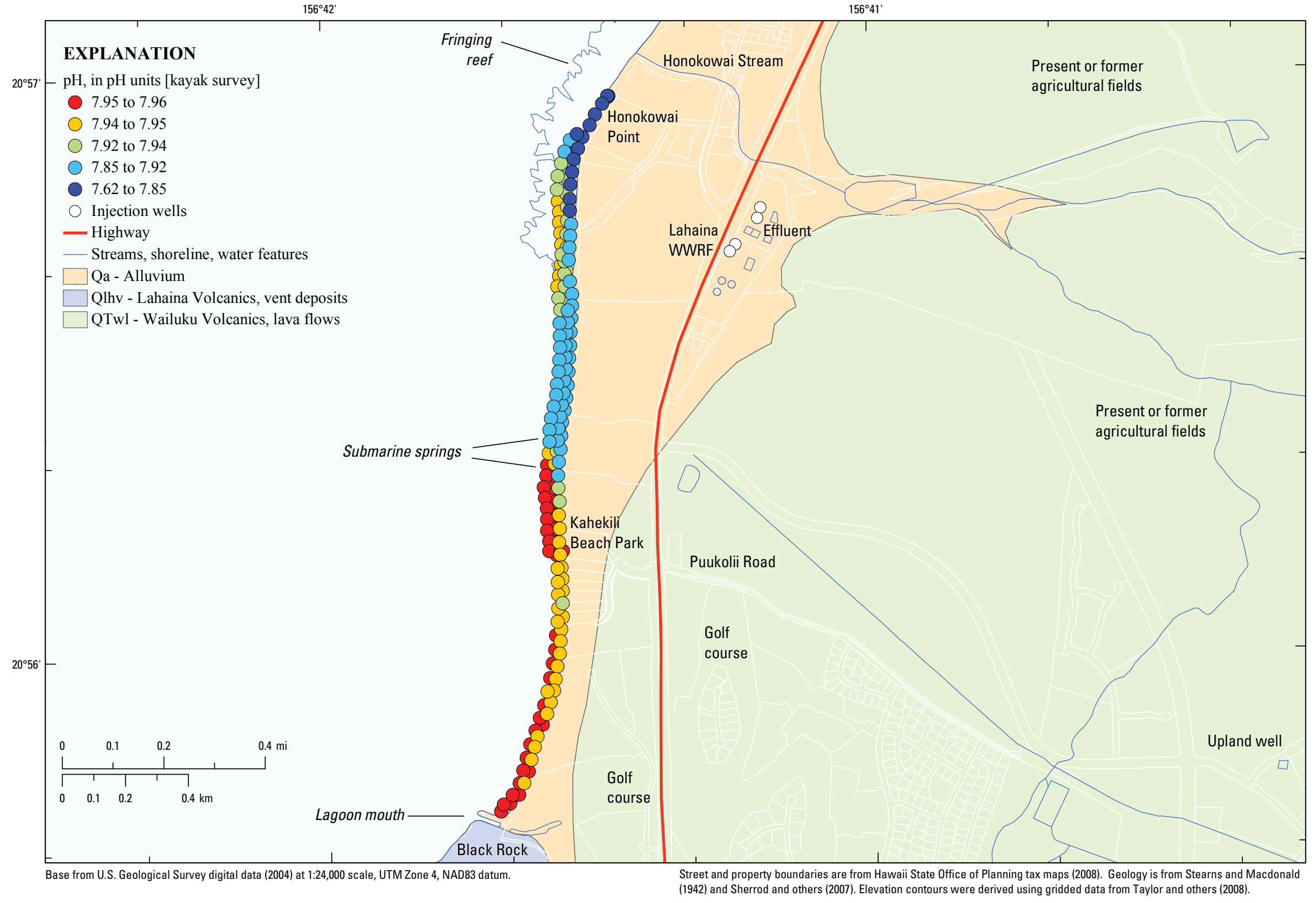

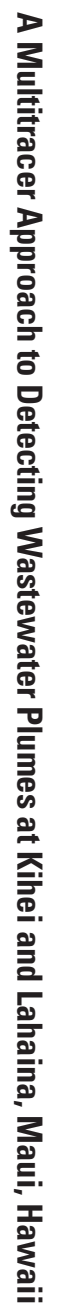

Figure A17. pH along kayak survey at Lahaina, Hawaii. 


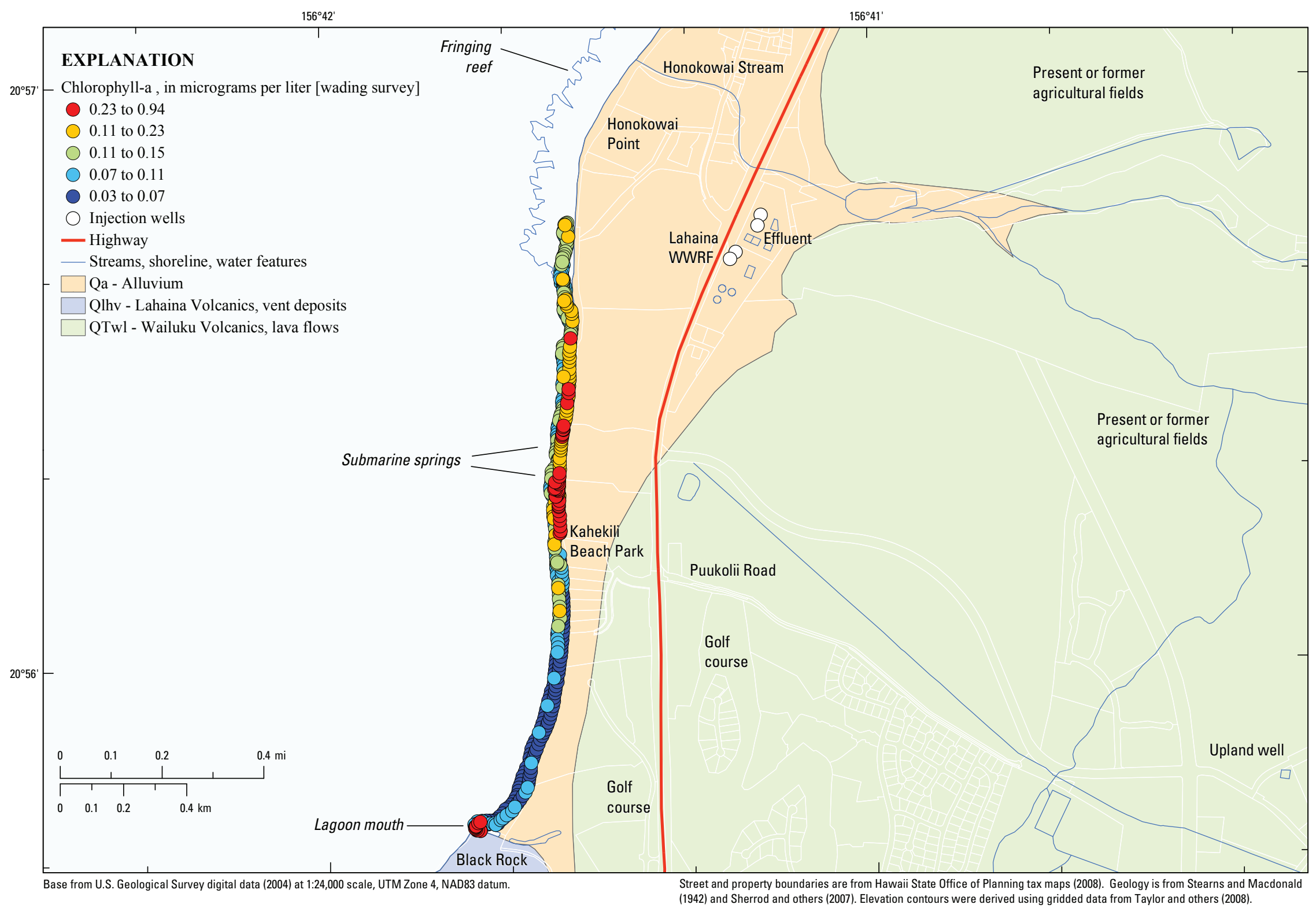

Figure A18. Chlorophyll-a concentration along wading survey at Lahaina, Hawaii. 
$156^{\circ} 42^{\prime}$

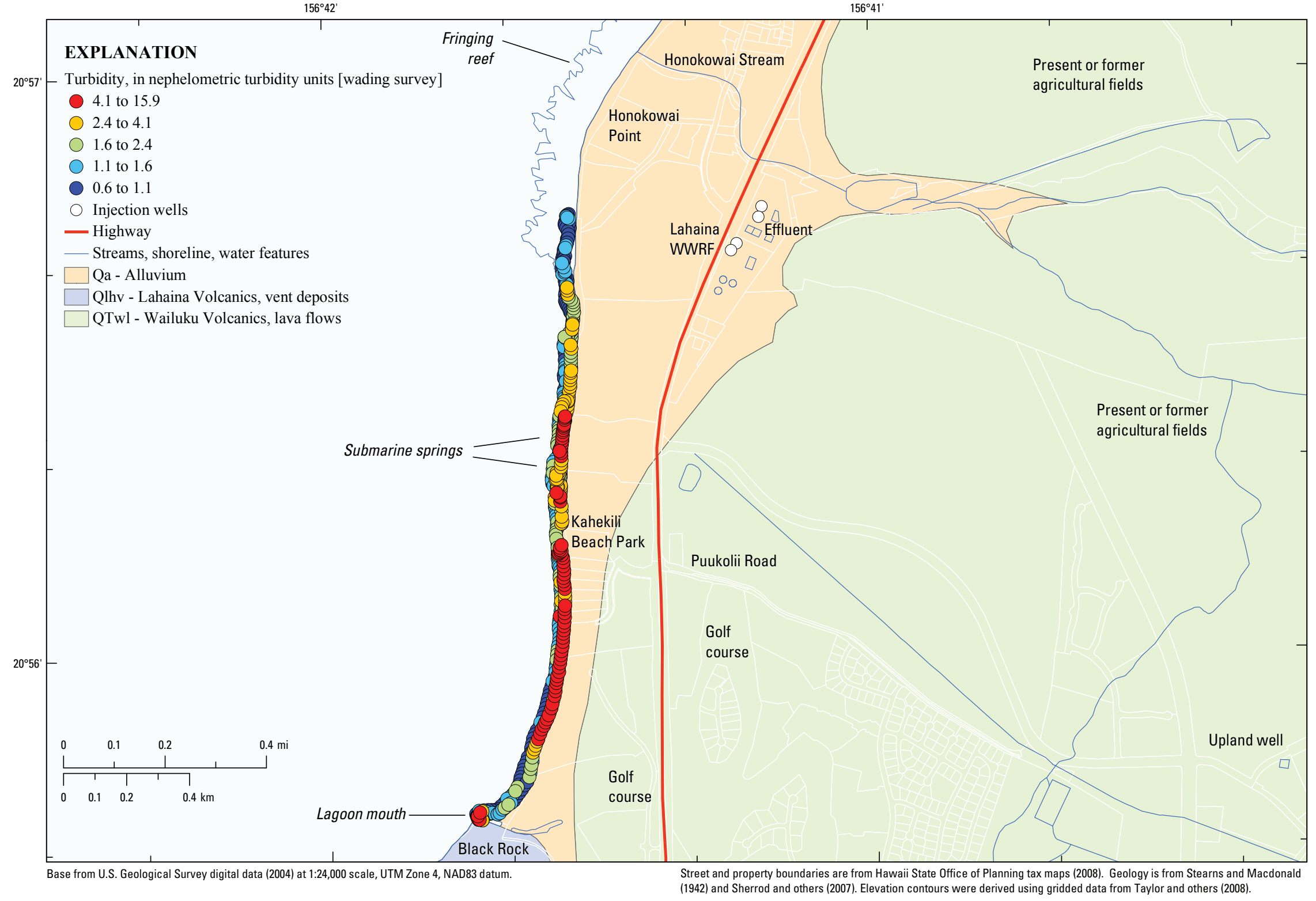

Figure A19. Turbidity along wading survey at Lahaina, Hawaii. 
Table A1. Nearshore wading survey with multiparameter water-quality sonde at Kihei, Hawaii, May 8, 2008.

[Abbreviations: ${ }^{\circ} \mathrm{C}$, degrees Celsius; $\mu \mathrm{S} / \mathrm{cm}$, microSiemens per centimeter; NTU, nephelometric turbidity units; mg/L, milligrams per liter; mm Hg, millimeters mercury; $\mu \mathrm{g} / \mathrm{L}$, micrograms per liter; salinity is dimensionless; chlorophyll values displaying an out-of-range flag were deleted]

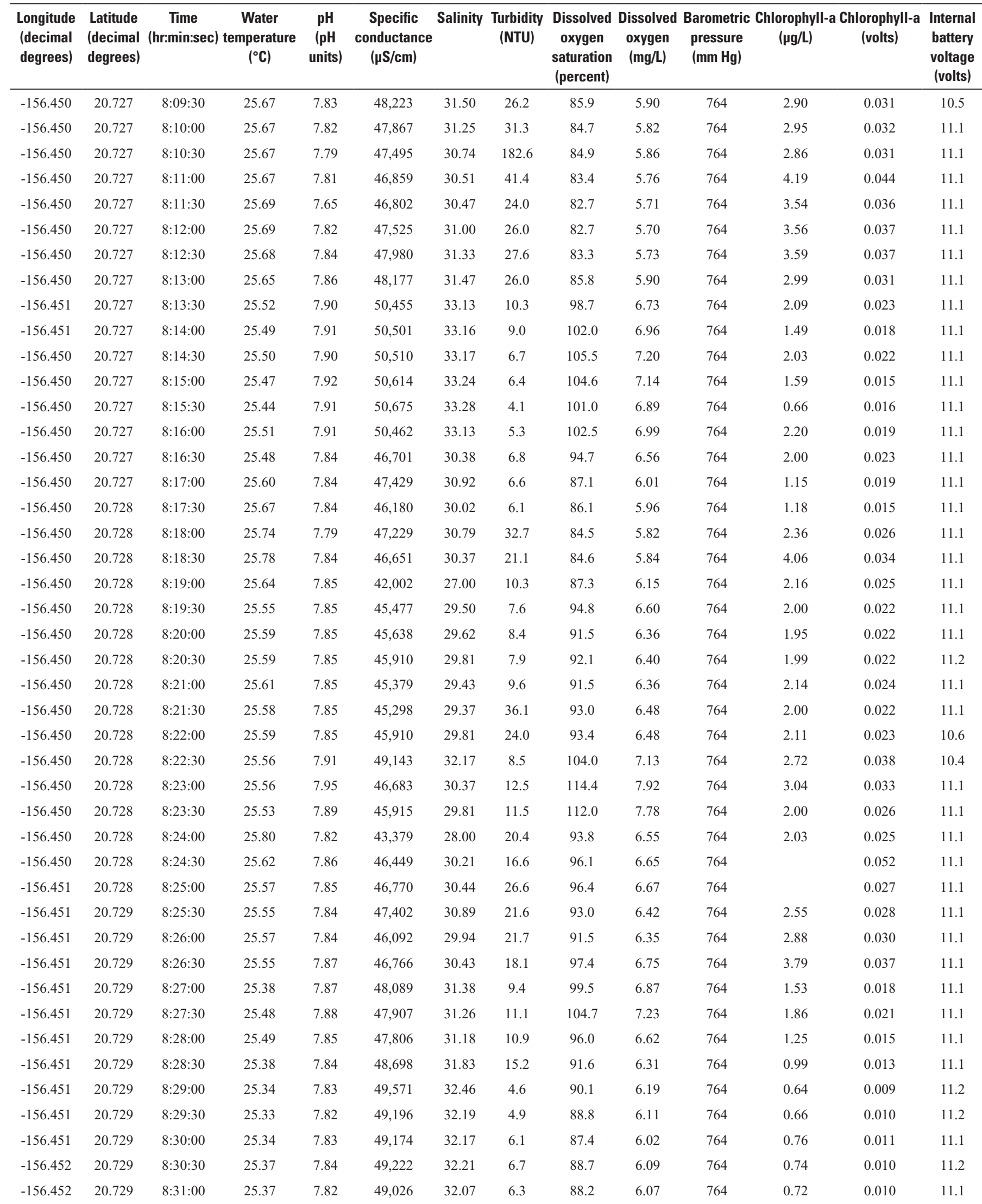


Table A1. Nearshore wading survey with multiparameter water-quality sonde at Kihei, Hawaii, May 8, 2008_Continued.

[Abbreviations: ${ }^{\circ} \mathrm{C}$, degrees Celsius; $\mu \mathrm{S} / \mathrm{cm}$, microSiemens per centimeter; NTU, nephelometric turbidity units; $\mathrm{mg} / \mathrm{L}$, milligrams per liter; mm Hg, millimeters mercury; $\mu \mathrm{g} / \mathrm{L}$, micrograms per liter; salinity is dimensionless; chlorophyll values displaying an out-of-range flag were deleted]

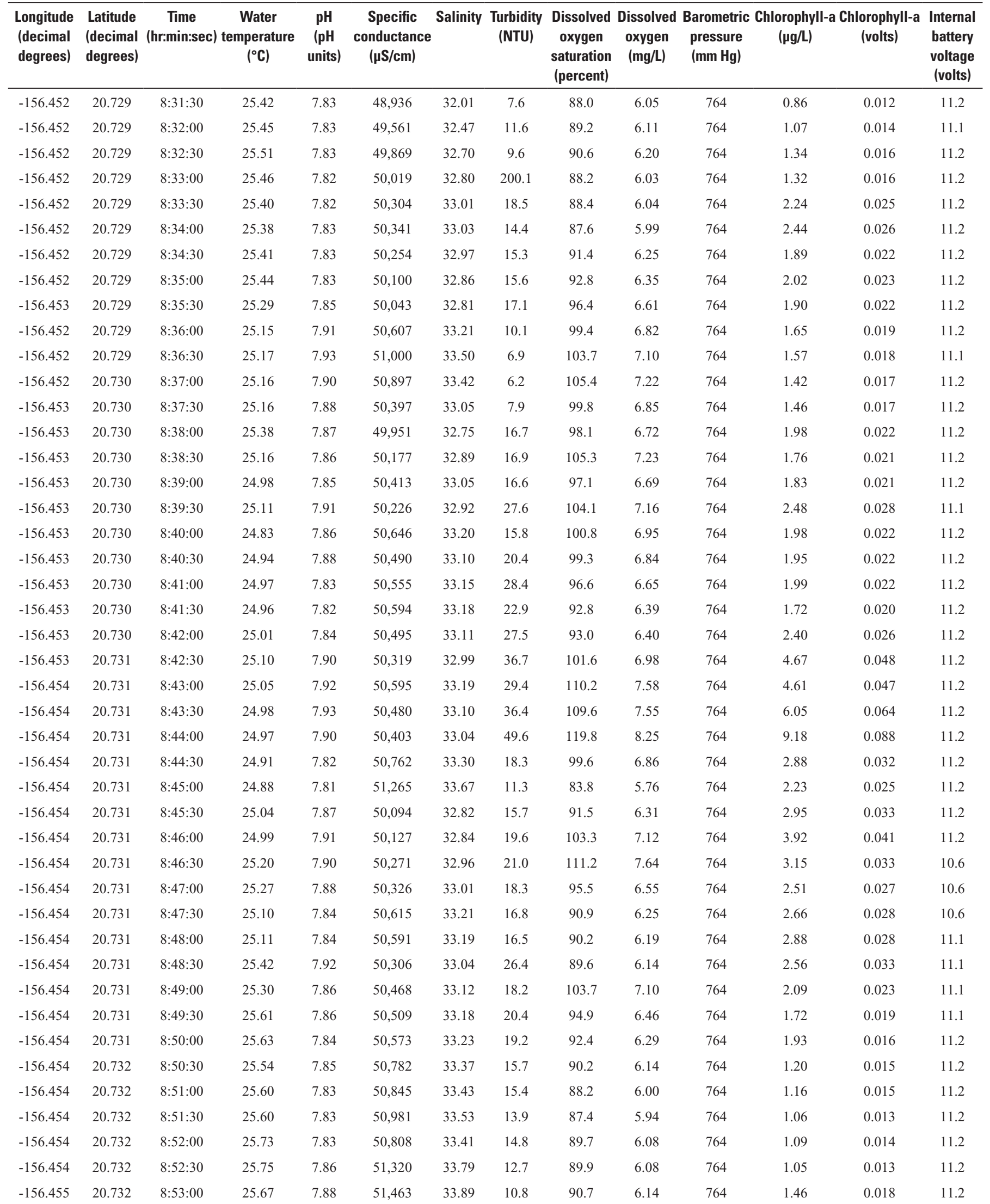


Table A1. Nearshore wading survey with multiparameter water-quality sonde at Kihei, Hawaii, May 8, 2008_Continued.

[Abbreviations: ${ }^{\circ} \mathrm{C}$, degrees Celsius; $\mu \mathrm{S} / \mathrm{cm}$, microSiemens per centimeter; NTU, nephelometric turbidity units; $\mathrm{mg} / \mathrm{L}$, milligrams per liter; mm Hg, millimeters mercury; $\mu \mathrm{g} / \mathrm{L}$, micrograms per liter; salinity is dimensionless; chlorophyll values displaying an out-of-range flag were deleted]

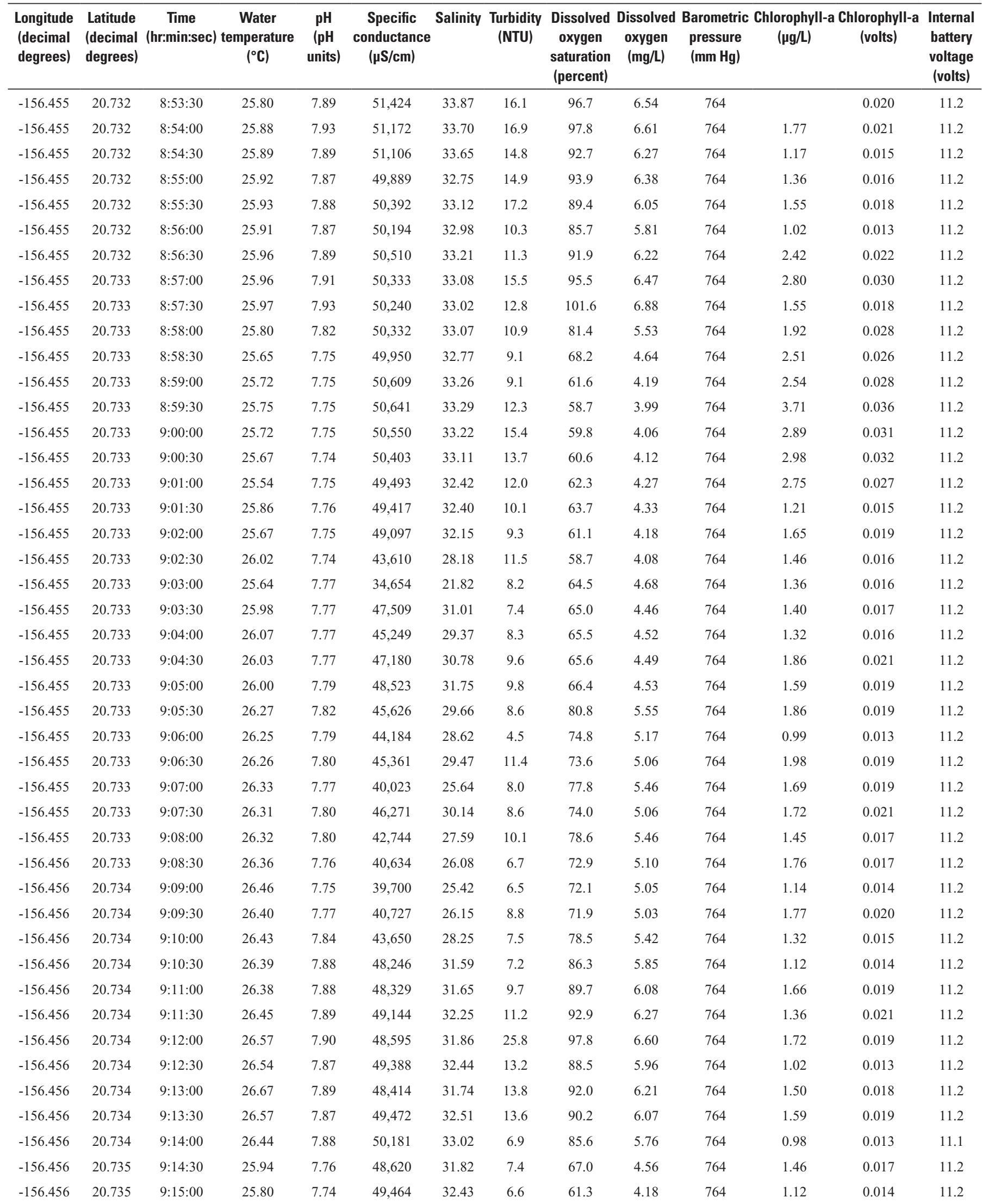


Table A1. Nearshore wading survey with multiparameter water-quality sonde at Kihei, Hawaii, May 8, 2008-Continued.

[Abbreviations: ${ }^{\circ} \mathrm{C}$, degrees Celsius; $\mu \mathrm{S} / \mathrm{cm}$, microSiemens per centimeter; NTU, nephelometric turbidity units; mg/L, milligrams per liter; mm Hg, millimeters mercury; $\mu \mathrm{g} / \mathrm{L}$, micrograms per liter; salinity is dimensionless; chlorophyll values displaying an out-of-range flag were deleted]

\begin{tabular}{|c|c|c|c|c|c|c|c|c|c|c|c|c|c|}
\hline $\begin{array}{l}\text { Longitude } \\
\text { (decimal } \\
\text { degrees) }\end{array}$ & $\begin{array}{l}\text { Latitude } \\
\text { (decimal } \\
\text { degrees) }\end{array}$ & $\begin{array}{c}\text { Time } \\
\text { (hr:min:sec) }\end{array}$ & $\begin{array}{c}\text { Water } \\
\text { temperature } \\
\left({ }^{\circ} \mathrm{C}\right)\end{array}$ & $\begin{array}{c}\text { pH } \\
\text { (pH } \\
\text { units) }\end{array}$ & $\begin{array}{c}\text { Specific } \\
\text { conductance } \\
(\mu \mathrm{S} / \mathrm{cm})\end{array}$ & Salinity & $\begin{array}{l}\text { Turbidity } \\
\text { (NTU) }\end{array}$ & $\begin{array}{l}\text { Dissolved } \\
\text { oxygen } \\
\text { saturation } \\
\text { (percent) }\end{array}$ & $\begin{array}{c}\text { Dissolved } \\
\text { oxygen } \\
\text { (mg/L) }\end{array}$ & $\begin{array}{c}\text { Barometric } \\
\text { pressure } \\
(\mathrm{mm} \mathrm{Hg})\end{array}$ & $\begin{array}{c}\text { Chlorophyll-a } \\
\text { ( } \mu \mathrm{g} / \mathrm{L})\end{array}$ & $\begin{array}{l}\text { a Chlorophyll-a } \\
\text { (volts) }\end{array}$ & $\begin{array}{c}\text { Internal } \\
\text { battery } \\
\text { voltage } \\
\text { (volts) }\end{array}$ \\
\hline-156.456 & 20.735 & 9:15:30 & 25.63 & 7.73 & 49,538 & 32.47 & 4.7 & 62.2 & 4.25 & 764 & 1.01 & 0.013 & 11.3 \\
\hline-156.456 & 20.735 & $9: 16: 00$ & 25.94 & 7.76 & 50,065 & 32.88 & 4.1 & 62.8 & 4.26 & 764 & 0.89 & 0.011 & 11.2 \\
\hline-156.456 & 20.735 & $9: 16: 30$ & 25.87 & 7.73 & 49,090 & 32.16 & 41.1 & 64.3 & 4.38 & 764 & 1.66 & 0.020 & 11.2 \\
\hline-156.456 & 20.735 & $9: 17: 00$ & 25.86 & 7.73 & 49,682 & 32.59 & 9.4 & 65.5 & 4.46 & 764 & 1.19 & 0.015 & 11.2 \\
\hline-156.456 & 20.735 & $9: 17: 30$ & 25.67 & 7.68 & 49,793 & 32.66 & 9.9 & 54.4 & 3.71 & 764 & 1.20 & 0.016 & 11.2 \\
\hline-156.456 & 20.735 & 9:18:00 & 26.17 & 7.80 & 49,012 & 32.13 & 16.9 & 68.3 & 4.63 & 764 & 0.87 & 0.012 & 11.2 \\
\hline-156.456 & 20.735 & $9: 18: 30$ & 25.99 & 7.79 & 48,981 & 32.09 & 11.9 & 73.0 & 4.97 & 764 & 1.01 & 0.013 & 11.2 \\
\hline-156.456 & 20.736 & 9:19:00 & 25.86 & 7.79 & 49,496 & 32.46 & 17.6 & 73.7 & 5.01 & 764 & 1.80 & 0.021 & 11.2 \\
\hline-156.456 & 20.736 & $9: 19: 30$ & 25.94 & 7.82 & 50,022 & 32.85 & 10.0 & 75.7 & 5.13 & 764 & 1.96 & 0.024 & 11.2 \\
\hline-156.456 & 20.736 & 9:20:00 & 26.11 & 7.86 & 50,001 & 32.85 & 8.2 & 89.8 & 6.07 & 764 & 1.30 & 0.016 & 11.2 \\
\hline-156.456 & 20.736 & $9: 20: 30$ & 26.15 & 7.82 & 49,955 & 32.82 & 8.7 & 80.5 & 5.44 & 764 & 1.11 & 0.014 & 11.2 \\
\hline-156.456 & 20.736 & $9: 21: 00$ & 26.20 & 7.84 & 48,253 & 31.57 & 13.3 & 78.9 & 5.37 & 764 & & 0.327 & 11.2 \\
\hline-156.456 & 20.736 & $9: 21: 30$ & 26.13 & 7.81 & 49,207 & 32.27 & 24.0 & 76.9 & 5.22 & 764 & 1.43 & 0.017 & 11.2 \\
\hline-156.456 & 20.736 & 9:22:00 & 26.08 & 7.82 & 49,712 & 32.64 & 4.8 & 73.8 & 5.00 & 764 & 0.62 & 0.009 & 11.2 \\
\hline-156.456 & 20.736 & $9: 22: 30$ & 26.07 & 7.82 & 49,124 & 32.20 & 4.6 & 74.7 & 5.07 & 764 & 0.69 & 0.010 & 11.3 \\
\hline-156.456 & 20.736 & 9:23:00 & 26.16 & 7.82 & 49,162 & 32.24 & 15.9 & 75.2 & 5.10 & 764 & 1.17 & 0.014 & 11.2 \\
\hline-156.456 & 20.736 & $9: 23: 30$ & 26.23 & 7.81 & 44,760 & 29.03 & 11.5 & 74.5 & 5.14 & 764 & 0.84 & 0.012 & 11.2 \\
\hline-156.456 & 20.736 & 9:24:00 & 25.74 & 7.79 & 38,456 & 24.49 & 13.3 & 73.8 & 5.26 & 764 & 454.89 & 4.367 & 11.2 \\
\hline-156.456 & 20.736 & $9: 24: 30$ & 25.64 & 7.77 & 39,288 & 25.07 & 4.6 & 72.9 & 5.20 & 764 & & 0.009 & 11.2 \\
\hline-156.456 & 20.736 & $9: 25: 00$ & 25.72 & 7.78 & 40,396 & 25.86 & 8.2 & 73.2 & 5.18 & 764 & 0.63 & 0.011 & 11.3 \\
\hline-156.456 & 20.736 & $9: 25: 30$ & 25.62 & 7.75 & 37,546 & 23.84 & 34.3 & 72.6 & 5.21 & 764 & & 0.444 & 11.2 \\
\hline-156.456 & 20.736 & $9: 26: 00$ & 25.58 & 7.75 & 37,304 & 23.67 & 20.1 & 72.1 & 5.18 & 764 & 1.03 & 0.013 & 11.2 \\
\hline-156.456 & 20.736 & $9: 26: 30$ & 25.47 & 7.75 & 41,607 & 26.71 & 4.3 & 74.0 & 5.24 & 764 & 0.43 & 0.008 & 11.3 \\
\hline-156.456 & 20.736 & 9:27:00 & 25.33 & 7.80 & 33,105 & 20.72 & 4.5 & 76.2 & 5.60 & 764 & 0.44 & 0.008 & 11.2 \\
\hline-156.456 & 20.736 & $9: 27: 30$ & 25.46 & 7.82 & 36,359 & 22.99 & 6.6 & 89.0 & 6.44 & 764 & 0.62 & 0.009 & 11.3 \\
\hline-156.456 & 20.736 & $9: 28: 00$ & 25.30 & 7.79 & 29,139 & 18.01 & 6.7 & 83.1 & 6.20 & 764 & 0.58 & 0.009 & 11.3 \\
\hline-156.456 & 20.737 & $9: 28: 30$ & 25.21 & 7.73 & 29,956 & 18.56 & 6.4 & 76.2 & 5.68 & 764 & 0.47 & 0.008 & 11.3 \\
\hline-156.456 & 20.737 & 9:29:00 & 25.59 & 7.84 & 42,611 & 27.43 & 6.8 & 80.5 & 5.66 & 764 & 0.61 & 0.009 & 11.3 \\
\hline-156.456 & 20.737 & $9: 29: 30$ & 26.00 & 7.88 & 48,422 & 31.68 & 6.2 & 93.6 & 6.39 & 764 & 0.90 & 0.012 & 11.3 \\
\hline-156.457 & 20.737 & 9:30:00 & 25.97 & 7.86 & 48,797 & 31.95 & 5.1 & 84.9 & 5.78 & 764 & 0.63 & 0.009 & 11.3 \\
\hline-156.457 & 20.737 & 9:30:30 & 26.02 & 7.86 & 47,942 & 31.33 & 8.0 & 79.9 & 5.46 & 764 & 0.50 & 0.009 & 11.2 \\
\hline-156.457 & 20.737 & $9: 31: 00$ & 25.96 & 7.88 & 48,099 & 31.44 & 7.9 & 80.4 & 5.49 & 764 & 0.52 & 0.008 & 11.3 \\
\hline-156.457 & 20.737 & $9: 31: 30$ & 25.93 & 7.87 & 48,492 & 31.73 & 6.8 & 82.7 & 5.64 & 764 & 0.50 & 0.008 & 11.3 \\
\hline-156.457 & 20.737 & 9:32:00 & 25.94 & 7.85 & 48,952 & 32.07 & 7.0 & 78.8 & 5.37 & 764 & 0.45 & 0.008 & 11.3 \\
\hline-156.457 & 20.737 & $9: 32: 30$ & 26.00 & 7.86 & 49,432 & 32.42 & 7.0 & 76.5 & 5.20 & 764 & 0.50 & 0.008 & 11.3 \\
\hline-156.457 & 20.737 & 9:33:00 & 26.01 & 7.85 & 47,905 & 31.30 & 8.9 & 79.1 & 5.41 & 764 & 0.54 & 0.009 & 11.3 \\
\hline-156.457 & 20.737 & $9: 33: 30$ & 26.17 & 7.89 & 48,673 & 31.88 & 7.9 & 86.2 & 5.86 & 764 & & 0.046 & 11.2 \\
\hline-156.457 & 20.738 & $9: 34: 00$ & 26.25 & 7.90 & 48,434 & 31.71 & 9.1 & 88.9 & 6.04 & 764 & 0.95 & 0.012 & 11.3 \\
\hline-156.457 & 20.738 & $9: 34: 30$ & 25.96 & 7.88 & 47,650 & 31.11 & 8.9 & 87.3 & 5.98 & 764 & 0.75 & 0.010 & 11.3 \\
\hline-156.457 & 20.738 & 9:35:00 & 25.98 & 7.90 & 47,789 & 31.22 & 11.5 & 90.5 & 6.19 & 764 & & 0.014 & 11.3 \\
\hline-156.457 & 20.738 & $9: 35: 30$ & 25.89 & 7.91 & 47,294 & 30.85 & 9.2 & 90.4 & 6.21 & 764 & 0.85 & 0.011 & 11.3 \\
\hline-156.457 & 20.738 & $9: 36: 00$ & 25.80 & 7.97 & 48,014 & 31.37 & 10.9 & 97.0 & 6.65 & 764 & 1.09 & 0.014 & 11.3 \\
\hline-156.457 & 20.738 & $9: 36: 30$ & 25.56 & 8.00 & 48,877 & 31.97 & 8.6 & 106.9 & 7.34 & 764 & 0.86 & 0.012 & 11.2 \\
\hline-156.457 & 20.738 & $9: 37: 00$ & 25.51 & 7.99 & 48,454 & 31.66 & 6.9 & 106.8 & 7.35 & 764 & 0.53 & 0.008 & 11.2 \\
\hline
\end{tabular}


Table A1. Nearshore wading survey with multiparameter water-quality sonde at Kihei, Hawaii, May 8, 2008-Continued.

[Abbreviations: ${ }^{\circ} \mathrm{C}$, degrees Celsius; $\mu \mathrm{S} / \mathrm{cm}$, microSiemens per centimeter; NTU, nephelometric turbidity units; $\mathrm{mg} / \mathrm{L}$, milligrams per liter; mm Hg, millimeters mercury; $\mu \mathrm{g} / \mathrm{L}$, micrograms per liter; salinity is dimensionless; chlorophyll values displaying an out-of-range flag were deleted]

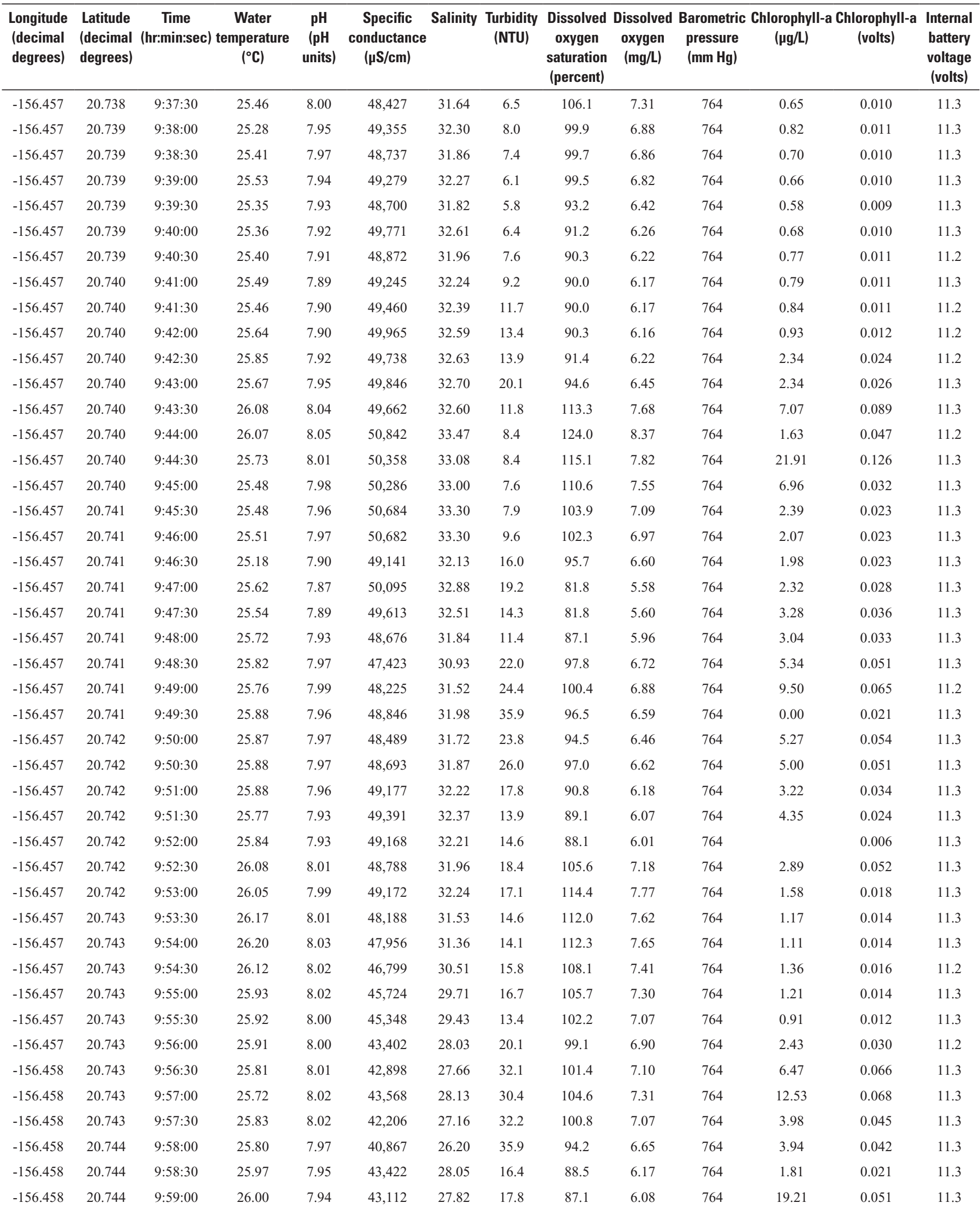


Table A1. Nearshore wading survey with multiparameter water-quality sonde at Kihei, Hawaii, May 8, 2008-Continued.

[Abbreviations: ${ }^{\circ} \mathrm{C}$, degrees Celsius; $\mu \mathrm{S} / \mathrm{cm}$, microSiemens per centimeter; NTU, nephelometric turbidity units; mg/L, milligrams per liter; mm Hg, millimeters mercury; $\mu \mathrm{g} / \mathrm{L}$, micrograms per liter; salinity is dimensionless; chlorophyll values displaying an out-of-range flag were deleted]

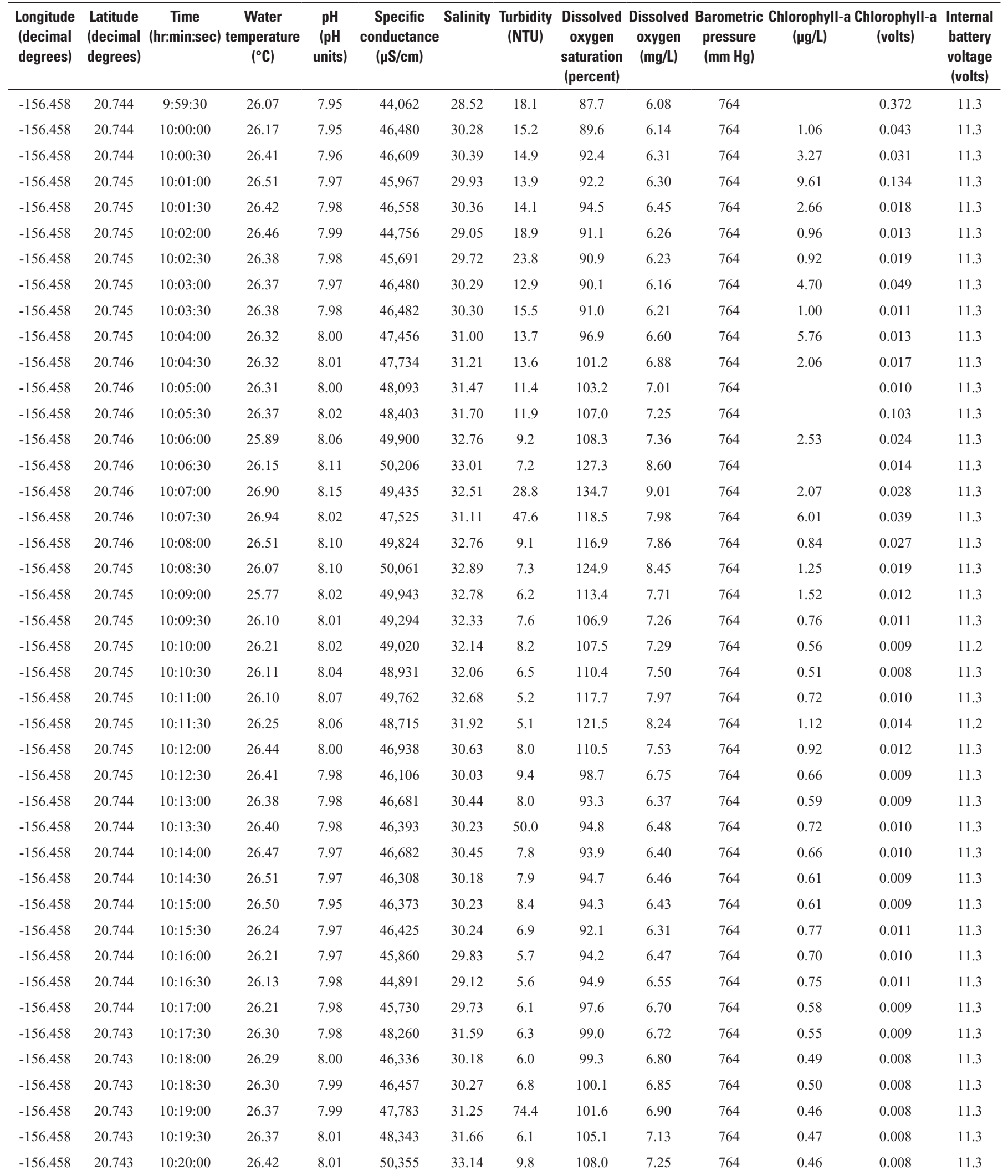


Table A1. Nearshore wading survey with multiparameter water-quality sonde at Kihei, Hawaii, May 8, 2008-Continued.

[Abbreviations: ${ }^{\circ} \mathrm{C}$, degrees Celsius; $\mu \mathrm{S} / \mathrm{cm}$, microSiemens per centimeter; NTU, nephelometric turbidity units; $\mathrm{mg} / \mathrm{L}$, milligrams per liter; mm Hg, millimeters mercury; $\mu \mathrm{g} / \mathrm{L}$, micrograms per liter; salinity is dimensionless; chlorophyll values displaying an out-of-range flag were deleted]

\begin{tabular}{|c|c|c|c|c|c|c|c|c|c|c|c|c|c|}
\hline $\begin{array}{l}\text { Longitude } \\
\text { (decimal } \\
\text { degrees) }\end{array}$ & $\begin{array}{l}\text { Latitude } \\
\text { (decimal } \\
\text { degrees) }\end{array}$ & $\begin{array}{c}\text { Time } \\
\text { (hr:min:sec) }\end{array}$ & $\begin{array}{c}\text { Water } \\
\text { temperature } \\
\left({ }^{\circ} \mathrm{C}\right)\end{array}$ & $\begin{array}{c}\text { pH } \\
\text { (pH } \\
\text { units) }\end{array}$ & $\begin{array}{c}\text { Specific } \\
\text { conductance } \\
(\mu \mathrm{S} / \mathrm{cm})\end{array}$ & Salinity & $\begin{array}{l}\text { Turbidity } \\
\text { (NTU) }\end{array}$ & $\begin{array}{l}\text { Dissolved } \\
\text { oxygen } \\
\text { saturation } \\
\text { (percent) }\end{array}$ & $\begin{array}{c}\text { Dissolved } \\
\text { oxygen } \\
\text { (mg/L) }\end{array}$ & $\begin{array}{c}\text { Barometric } \\
\text { pressure } \\
(\mathrm{mm} \mathrm{Hg})\end{array}$ & $\begin{array}{l}\text { Chlorophyll-a } \\
\text { ( } \mu \mathrm{g} / \mathrm{L})\end{array}$ & $\begin{array}{l}\text { a Chlorophyll-a } \\
\text { (volts) }\end{array}$ & $\begin{array}{c}\text { Internal } \\
\text { battery } \\
\text { voltage } \\
\text { (volts) }\end{array}$ \\
\hline-156.458 & 20.742 & $10: 21: 00$ & 26.38 & 8.04 & 50,491 & 33.24 & 6.6 & 113.7 & 7.64 & 764 & 0.46 & 0.008 & 11.3 \\
\hline-156.457 & 20.742 & $10: 21: 30$ & 26.36 & 8.03 & 50,350 & 33.13 & 8.5 & 116.1 & 7.81 & 764 & 0.61 & 0.009 & 11.3 \\
\hline-156.457 & 20.742 & $10: 23: 00$ & 26.34 & 8.01 & 49,877 & 32.78 & 7.5 & 114.5 & 7.72 & 764 & 0.71 & 0.010 & 11.3 \\
\hline-156.457 & 20.742 & $10: 23: 30$ & 26.30 & 7.98 & 50,003 & 32.87 & 6.4 & 101.7 & 6.86 & 764 & 0.70 & 0.010 & 11.3 \\
\hline-156.457 & 20.742 & $10: 24: 00$ & 26.28 & 7.98 & 49,871 & 32.77 & 6.5 & 102.1 & 6.89 & 764 & 0.95 & 0.012 & 10.6 \\
\hline-156.457 & 20.741 & $10: 24: 30$ & 26.38 & 8.00 & 49,853 & 32.77 & 6.7 & 104.4 & 7.03 & 764 & 0.94 & 0.012 & 10.7 \\
\hline-156.457 & 20.741 & $10: 26: 30$ & 26.18 & 8.01 & 50,655 & 33.34 & 5.5 & 108.7 & 7.33 & 764 & 1.17 & 0.015 & 10.6 \\
\hline-156.457 & 20.740 & $10: 27: 00$ & 26.25 & 8.06 & 50,961 & 33.58 & 11.5 & 125.0 & 8.40 & 764 & 1.72 & 0.015 & 10.6 \\
\hline-156.457 & 20.740 & $10: 27: 30$ & 26.32 & 8.02 & 51,164 & 33.73 & 5.0 & 123.8 & 8.31 & 764 & 1.13 & 0.014 & 10.6 \\
\hline-156.457 & 20.740 & 10:28:00 & 26.42 & 7.98 & 51,376 & 33.90 & 5.3 & 114.7 & 7.67 & 764 & 1.07 & 0.013 & 11.1 \\
\hline-156.457 & 20.740 & $10: 28: 30$ & 26.32 & 7.99 & 51,302 & 33.83 & 5.8 & 111.1 & 7.45 & 764 & 0.84 & 0.011 & 11.2 \\
\hline-156.457 & 20.740 & 10:29:00 & 26.08 & 8.00 & 50,997 & 33.59 & 5.4 & 115.1 & 7.75 & 764 & 0.49 & 0.008 & 11.2 \\
\hline-156.457 & 20.740 & $10: 29: 30$ & 26.14 & 8.01 & 50,787 & 33.44 & 4.1 & 119.5 & 8.05 & 764 & 0.46 & 0.008 & 11.2 \\
\hline-156.457 & 20.740 & 10:30:00 & 26.17 & 7.99 & 50,500 & 33.23 & 3.9 & 119.5 & 8.06 & 764 & 0.44 & 0.008 & 11.2 \\
\hline-156.457 & 20.739 & $10: 30: 30$ & 26.24 & 7.98 & 50,228 & 33.03 & 4.3 & 114.6 & 7.73 & 764 & 0.38 & 0.007 & 11.2 \\
\hline-156.457 & 20.739 & 10:31:00 & 26.33 & 7.97 & 50,060 & 32.92 & 5.8 & 110.2 & 7.42 & 764 & 0.55 & 0.008 & 11.2 \\
\hline-156.457 & 20.738 & $10: 34: 30$ & 26.52 & 7.98 & 48,583 & 31.85 & 3.7 & 113.4 & 7.66 & 764 & 0.37 & 0.008 & 10.5 \\
\hline-156.457 & 20.738 & $10: 35: 00$ & 26.46 & 7.94 & 47,556 & 31.09 & 3.9 & 106.0 & 7.20 & 764 & 0.29 & 0.006 & 10.5 \\
\hline-156.457 & 20.738 & $10: 35: 30$ & 26.46 & 7.94 & 47,062 & 30.73 & 3.3 & 105.1 & 7.15 & 764 & 0.29 & 0.006 & 10.6 \\
\hline-156.457 & 20.738 & 10:36:00 & 26.44 & 7.92 & 45,857 & 29.85 & 3.4 & 102.7 & 7.03 & 764 & 0.29 & 0.006 & 10.6 \\
\hline-156.457 & 20.737 & $10: 36: 30$ & 26.50 & 7.94 & 45,359 & 29.49 & 4.5 & 102.0 & 6.99 & 764 & 0.33 & 0.006 & 10.6 \\
\hline-156.457 & 20.737 & $10: 37: 00$ & 26.50 & 7.95 & 45,421 & 29.53 & 5.0 & 104.0 & 7.12 & 764 & 0.38 & 0.007 & 10.6 \\
\hline-156.457 & 20.737 & $10: 37: 30$ & 26.48 & 7.89 & 45,288 & 29.44 & 4.2 & 102.5 & 7.03 & 764 & 0.32 & 0.006 & 10.6 \\
\hline-156.457 & 20.737 & $10: 38: 00$ & 26.43 & 7.87 & 43,626 & 28.23 & 3.0 & 98.2 & 6.78 & 764 & 0.30 & 0.006 & 10.5 \\
\hline-156.457 & 20.737 & $10: 38: 30$ & 26.37 & 7.87 & 42,371 & 27.32 & 2.3 & 94.9 & 6.59 & 764 & 0.14 & 0.005 & 10.6 \\
\hline-156.457 & 20.737 & 10:39:00 & 26.44 & 7.87 & 43,432 & 28.09 & 2.1 & 96.0 & 6.63 & 764 & 0.34 & 0.006 & 10.6 \\
\hline-156.457 & 20.737 & $10: 39: 30$ & 26.40 & 7.87 & 40,701 & 26.13 & 2.3 & 96.7 & 6.75 & 764 & 0.28 & 0.006 & 10.4 \\
\hline-156.457 & 20.737 & 10:40:00 & 26.37 & 7.87 & 40,763 & 26.17 & 2.2 & 96.7 & 6.76 & 764 & 0.27 & 0.006 & 11.1 \\
\hline-156.457 & 20.737 & $10: 40: 30$ & 26.44 & 7.88 & 41,263 & 26.53 & 2.2 & 97.3 & 6.78 & 764 & 0.23 & 0.006 & 11.1 \\
\hline-156.457 & 20.736 & 10:41:00 & 26.44 & 7.87 & 40,342 & 25.87 & 2.5 & 98.9 & 6.92 & 764 & 0.66 & 0.009 & 11.1 \\
\hline
\end{tabular}


Table A1. Nearshore wading survey with multiparameter water-quality sonde at Kihei, Hawaii, May 8, 2008-Continued.

[Abbreviations: ${ }^{\circ} \mathrm{C}$, degrees Celsius; $\mu \mathrm{S} / \mathrm{cm}$, microSiemens per centimeter; NTU, nephelometric turbidity units; mg/L, milligrams per liter; mm Hg, millimeters mercury; $\mu \mathrm{g} / \mathrm{L}$, micrograms per liter; salinity is dimensionless; chlorophyll values displaying an out-of-range flag were deleted]

\begin{tabular}{|c|c|c|c|c|c|c|c|c|c|c|c|c|c|}
\hline $\begin{array}{l}\text { Longitude } \\
\text { (decimal } \\
\text { degrees) }\end{array}$ & $\begin{array}{l}\text { Latitude } \\
\text { (decimal } \\
\text { degrees) }\end{array}$ & $\begin{array}{c}\text { Time } \\
\text { (hr:min:sec) }\end{array}$ & $\begin{array}{c}\text { Water } \\
\text { temperature } \\
\left({ }^{\circ} \mathrm{C}\right)\end{array}$ & $\begin{array}{c}\text { pH } \\
\text { (pH } \\
\text { units) }\end{array}$ & $\begin{array}{c}\text { Specific } \\
\text { conductance } \\
(\mu \mathrm{S} / \mathrm{cm})\end{array}$ & Salinity & $\begin{array}{l}\text { Turbidity } \\
\text { (NTU) }\end{array}$ & $\begin{array}{l}\text { Dissolved } \\
\text { oxygen } \\
\text { saturation } \\
\text { (percent) }\end{array}$ & $\begin{array}{c}\text { Dissolved } \\
\text { oxygen } \\
\text { (mg/L) }\end{array}$ & $\begin{array}{l}\text { Barometric } \\
\text { pressure } \\
(\mathrm{mm} \mathrm{Hg})\end{array}$ & $\begin{array}{l}\text { Chlorophyll-a } \\
(\mu \mathrm{g} / \mathrm{L})\end{array}$ & $\begin{array}{l}\text { Chlorophyll-a } \\
\text { (volts) }\end{array}$ & $\begin{array}{c}\text { Internal } \\
\text { battery } \\
\text { voltage } \\
\text { (volts) }\end{array}$ \\
\hline-156.457 & 20.736 & $10: 42: 00$ & 26.19 & 7.86 & 37,515 & 23.85 & 2.3 & 98.9 & 7.03 & 764 & 0.25 & 0.006 & 11.2 \\
\hline-156.457 & 20.736 & $10: 42: 30$ & 26.20 & 7.88 & 37,211 & 23.64 & 2.5 & 101.4 & 7.21 & 764 & 0.34 & 0.007 & 11.2 \\
\hline-156.457 & 20.736 & $10: 44: 00$ & 26.41 & 7.90 & 39,587 & 25.33 & 2.9 & 103.5 & 7.27 & 764 & 0.32 & 0.006 & 11.2 \\
\hline-156.457 & 20.736 & $10: 44: 30$ & 26.29 & 7.89 & 39,454 & 25.23 & 2.6 & 102.4 & 7.21 & 764 & 0.26 & 0.006 & 11.2 \\
\hline-156.457 & 20.736 & $10: 45: 00$ & 26.44 & 7.92 & 40,179 & 25.76 & 2.7 & 104.6 & 7.29 & 764 & 0.30 & 0.006 & 10.4 \\
\hline-156.457 & 20.736 & $10: 45: 30$ & 26.39 & 7.92 & 41,167 & 26.46 & 3.3 & 110.4 & 7.71 & 764 & 0.44 & 0.008 & 10.4 \\
\hline-156.456 & 20.736 & $10: 47: 30$ & 26.25 & 7.89 & 38,359 & 24.45 & 3.3 & 103.3 & 7.31 & 764 & 0.32 & 0.006 & 11.2 \\
\hline-156.456 & 20.736 & $10: 48: 00$ & 26.13 & 7.88 & 33,579 & 21.10 & 3.1 & 101.0 & 7.30 & 764 & 0.28 & 0.006 & 11.2 \\
\hline-156.456 & 20.735 & $10: 48: 30$ & 26.35 & 7.90 & 36,887 & 23.42 & 2.5 & 102.1 & 7.25 & 764 & 0.33 & 0.006 & 11.2 \\
\hline-156.456 & 20.735 & 10:49:00 & 26.96 & 7.90 & 44,320 & 28.77 & 3.3 & 103.8 & 7.08 & 764 & 0.37 & 0.007 & 11.2 \\
\hline-156.456 & 20.735 & $10: 49: 30$ & 27.08 & 7.94 & 47,404 & 31.03 & 4.4 & 113.8 & 7.65 & 764 & 0.45 & 0.008 & 11.2 \\
\hline-156.456 & 20.735 & 10:50:00 & 27.00 & 7.97 & 49,119 & 32.28 & 4.4 & 113.0 & 7.55 & 764 & 0.44 & 0.007 & 11.2 \\
\hline-156.456 & 20.735 & $10: 50: 30$ & 27.03 & 7.99 & 49,031 & 32.22 & 3.7 & 119.8 & 8.01 & 764 & 0.50 & 0.008 & 11.2 \\
\hline-156.456 & 20.735 & 10:51:00 & 27.06 & 7.99 & 48,750 & 32.02 & 5.4 & 120.4 & 8.05 & 764 & 0.43 & 0.007 & 10.6 \\
\hline-156.456 & 20.734 & $10: 51: 30$ & 27.09 & 7.99 & 48,407 & 31.77 & 6.4 & 119.5 & 8.00 & 764 & 0.38 & 0.007 & 10.6 \\
\hline-156.456 & 20.734 & 10:52:00 & 27.07 & 7.99 & 48,060 & 31.51 & 6.3 & 119.1 & 7.99 & 764 & 0.44 & 0.007 & 10.6 \\
\hline-156.455 & 20.733 & $10: 55: 30$ & 27.05 & 7.99 & 42,975 & 27.81 & 8.6 & 123.6 & 8.46 & 764 & 0.61 & 0.009 & 11.2 \\
\hline-156.455 & 20.733 & $10: 56: 00$ & 27.04 & 8.01 & 42,487 & 27.45 & 7.0 & 122.2 & 8.39 & 764 & 0.68 & 0.010 & 11.2 \\
\hline-156.455 & 20.733 & $10: 56: 30$ & 27.04 & 8.00 & 42,188 & 27.24 & 4.6 & 118.6 & 8.15 & 764 & 0.62 & 0.010 & 11.2 \\
\hline-156.455 & 20.733 & $10: 57: 00$ & 27.11 & 8.04 & 43,962 & 28.52 & 5.2 & 125.2 & 8.53 & 764 & 0.80 & 0.011 & 11.2 \\
\hline-156.455 & 20.733 & $10: 57: 30$ & 26.96 & 8.03 & 45,968 & 29.97 & 6.0 & 125.1 & 8.48 & 764 & 0.87 & 0.012 & 11.2 \\
\hline-156.455 & 20.732 & 10:58:00 & 26.91 & 8.00 & 48,911 & 32.12 & 8.4 & 120.8 & 8.10 & 764 & 0.97 & 0.013 & 11.2 \\
\hline-156.455 & 20.732 & $10: 58: 30$ & 26.71 & 7.99 & 50,027 & 32.93 & 7.5 & 117.5 & 7.86 & 764 & 0.56 & 0.009 & 10.7 \\
\hline-156.455 & 20.732 & 10:59:00 & 26.70 & 8.00 & 49,809 & 32.77 & 8.8 & 120.7 & 8.09 & 764 & 0.44 & 0.007 & 10.6 \\
\hline-156.455 & 20.732 & 10:59:30 & 26.73 & 8.01 & 49,451 & 32.50 & 6.4 & 125.2 & 8.40 & 764 & 0.57 & 0.009 & 10.6 \\
\hline-156.455 & 20.732 & 11:00:00 & 27.04 & 8.06 & 49,384 & 32.48 & 8.8 & 135.2 & 9.02 & 764 & 0.81 & 0.011 & 10.5 \\
\hline-156.454 & 20.732 & 11:00:30 & 26.69 & 8.03 & 48,019 & 31.45 & 9.7 & 135.2 & 9.12 & 764 & 0.90 & 0.012 & 10.5 \\
\hline-156.454 & 20.732 & 11:01:00 & 26.90 & 8.08 & 49,365 & 32.46 & 10.0 & 134.3 & 8.99 & 764 & 0.82 & 0.011 & 10.4 \\
\hline-156.454 & 20.732 & $11: 01: 30$ & 26.58 & 8.06 & 49,714 & 32.69 & 10.6 & 138.3 & 9.29 & 764 & 1.04 & 0.013 & 10.5 \\
\hline-156.454 & 20.731 & 11:02:00 & 26.79 & 8.10 & 50,192 & 33.06 & 12.3 & 140.5 & 9.39 & 764 & 1.54 & 0.018 & 10.4 \\
\hline-156.454 & 20.731 & $11: 02: 30$ & 26.72 & 8.08 & 50,122 & 33.00 & 13.2 & 134.7 & 9.01 & 764 & 1.31 & 0.016 & 10.5 \\
\hline-156.454 & 20.731 & 11:03:00 & 26.88 & 8.10 & 49,822 & 32.79 & 16.9 & 136.1 & 9.09 & 764 & 1.99 & 0.022 & 10.5 \\
\hline
\end{tabular}


Table A1. Nearshore wading survey with multiparameter water-quality sonde at Kihei, Hawaii, May 8, 2008-Continued.

[Abbreviations: ${ }^{\circ} \mathrm{C}$, degrees Celsius; $\mu \mathrm{S} / \mathrm{cm}$, microSiemens per centimeter; NTU, nephelometric turbidity units; $\mathrm{mg} / \mathrm{L}$, milligrams per liter; mm Hg, millimeters mercury; $\mu \mathrm{g} / \mathrm{L}$, micrograms per liter; salinity is dimensionless; chlorophyll values displaying an out-of-range flag were deleted]

\begin{tabular}{|c|c|c|c|c|c|c|c|c|c|c|c|c|c|}
\hline $\begin{array}{l}\text { Longitude } \\
\text { (decimal } \\
\text { degrees) }\end{array}$ & $\begin{array}{l}\text { Latitude } \\
\text { (decimal } \\
\text { degrees) }\end{array}$ & $\begin{array}{c}\text { Time } \\
\text { (hr:min:sec) }\end{array}$ & $\begin{array}{c}\text { Water } \\
\text { temperature } \\
\left({ }^{\circ} \mathrm{C}\right)\end{array}$ & $\begin{array}{c}\text { pH } \\
\text { (pH } \\
\text { units) }\end{array}$ & $\begin{array}{c}\text { Specific } \\
\text { conductance } \\
(\mu \mathrm{S} / \mathrm{cm})\end{array}$ & Salinity & $\begin{array}{l}\text { Turbidity } \\
\text { (NTU) }\end{array}$ & $\begin{array}{l}\text { Dissolved } \\
\text { oxygen } \\
\text { saturation } \\
\text { (percent) }\end{array}$ & $\begin{array}{c}\text { Dissolved } \\
\text { oxygen } \\
\text { (mg/L) }\end{array}$ & $\begin{array}{l}\text { Barometric } \\
\text { pressure } \\
(\mathrm{mm} \mathrm{Hg})\end{array}$ & $\begin{array}{l}\text { Chlorophyll-a } \\
\qquad(\mu \mathrm{g} / \mathrm{L})\end{array}$ & $\begin{array}{l}\text { a Chlorophyll-a } \\
\text { (volts) }\end{array}$ & $\begin{array}{c}\text { Internal } \\
\text { battery } \\
\text { voltage } \\
\text { (volts) }\end{array}$ \\
\hline-156.454 & 20.731 & 11:04:00 & 26.71 & 8.08 & 50,240 & 33.09 & 12.3 & 141.6 & 9.47 & 764 & 1.38 & 0.016 & 11.1 \\
\hline-156.454 & 20.731 & $11: 04: 30$ & 26.81 & 8.12 & 50,093 & 32.99 & 12.0 & 148.5 & 9.92 & 764 & 1.08 & 0.014 & 11.1 \\
\hline-156.454 & 20.731 & 11:06:00 & 26.87 & 8.08 & 51,568 & 34.09 & 4.7 & 136.2 & 9.03 & 764 & 0.66 & 0.009 & 11.1 \\
\hline-156.454 & 20.730 & $11: 06: 30$ & 26.84 & 8.03 & 51,669 & 34.16 & 5.4 & 125.7 & 8.34 & 764 & 0.54 & 0.008 & 11.1 \\
\hline-156.454 & 20.730 & 11:07:00 & 26.80 & 8.04 & 51,770 & 34.23 & 6.1 & 119.6 & 7.94 & 764 & 0.50 & 0.008 & 11.1 \\
\hline-156.454 & 20.730 & $11: 07: 30$ & 26.97 & 8.13 & 51,475 & 34.03 & 391.5 & 133.4 & 8.84 & 764 & 0.57 & 0.009 & 11.1 \\
\hline-156.453 & 20.730 & 11:09:30 & 26.91 & 8.23 & 50,700 & 33.44 & 6.8 & 193.9 & 12.90 & 764 & 1.35 & 0.014 & 10.6 \\
\hline-156.453 & 20.730 & 11:10:00 & 26.94 & 8.24 & 50,682 & 33.43 & 6.3 & 196.6 & 13.07 & 764 & 1.01 & 0.013 & 11.0 \\
\hline-156.453 & 20.730 & $11: 10: 30$ & 26.95 & 8.25 & 50,734 & 33.47 & 7.0 & 197.9 & 13.15 & 764 & 1.04 & 0.013 & 11.1 \\
\hline-156.453 & 20.730 & 11:11:00 & 27.02 & 8.25 & 50,838 & 33.56 & 6.3 & 189.4 & 12.57 & 764 & 0.98 & 0.013 & 11.1 \\
\hline-156.453 & 20.730 & $11: 11: 30$ & 26.99 & 8.27 & 51,430 & 33.99 & 5.5 & 190.6 & 12.62 & 764 & 0.91 & 0.012 & 11.1 \\
\hline-156.453 & 20.729 & 11:12:00 & 27.10 & 8.27 & 51,416 & 34.00 & 5.7 & 189.6 & 12.53 & 764 & 0.87 & 0.012 & 11.1 \\
\hline-156.453 & 20.729 & $11: 12: 30$ & 27.14 & 8.28 & 51,426 & 34.01 & 6.5 & 198.5 & 13.11 & 764 & 0.99 & 0.013 & 11.2 \\
\hline-156.452 & 20.729 & 11:13:00 & 27.20 & 8.22 & 51,236 & 33.87 & 6.5 & 182.5 & 12.05 & 764 & 0.78 & 0.011 & 11.2 \\
\hline-156.452 & 20.729 & $11: 13: 30$ & 27.18 & 8.26 & 51,327 & 33.94 & 5.4 & 173.6 & 11.47 & 764 & 0.77 & 0.011 & 11.2 \\
\hline-156.452 & 20.729 & $11: 14: 00$ & 27.20 & 8.23 & 50,939 & 33.65 & 25.0 & 188.4 & 12.46 & 764 & 0.88 & 0.012 & 11.2 \\
\hline-156.451 & 20.728 & $11: 17: 30$ & 27.08 & 8.15 & 49,565 & 32.62 & 12.1 & 159.6 & 10.63 & 764 & 0.99 & 0.013 & 11.2 \\
\hline-156.451 & 20.728 & 11:18:00 & 27.31 & 8.03 & 49,394 & 32.52 & 10.5 & 134.2 & 8.92 & 764 & 0.94 & 0.012 & 11.2 \\
\hline-156.451 & 20.728 & 11:18:30 & 27.27 & 8.02 & 49,529 & 32.61 & 12.0 & 114.4 & 7.60 & 764 & 0.96 & 0.010 & 11.2 \\
\hline-156.451 & 20.728 & 11:19:00 & 27.66 & 8.06 & 49,062 & 32.30 & 10.8 & 115.5 & 7.63 & 764 & 0.74 & 0.010 & 11.2 \\
\hline-156.450 & 20.728 & $11: 19: 30$ & 27.15 & 8.08 & 48,951 & 32.17 & 14.4 & 124.2 & 8.28 & 764 & 0.63 & 0.010 & 10.7 \\
\hline-156.450 & 20.728 & $11: 20: 00$ & 26.90 & 8.08 & 48,905 & 32.12 & 80.2 & 135.3 & 9.07 & 764 & 0.71 & 0.010 & 10.6 \\
\hline-156.450 & 20.728 & $11: 20: 30$ & 26.90 & 8.08 & 48,530 & 31.84 & 7.0 & 127.8 & 8.58 & 764 & 0.56 & 0.009 & 10.6 \\
\hline-156.450 & 20.728 & $11: 21: 00$ & 26.83 & 8.08 & 48,615 & 31.90 & 6.6 & 129.1 & 8.68 & 764 & 0.63 & 0.009 & 10.5 \\
\hline-156.450 & 20.727 & $11: 21: 30$ & 27.71 & 8.12 & 44,552 & 29.00 & 5.6 & 141.4 & 9.51 & 764 & 0.63 & 0.010 & 10.4 \\
\hline-156.460 & 20.755 & $12: 07: 30$ & 27.98 & 8.22 & 46,037 & 30.10 & 14.8 & 187.3 & 12.47 & 764 & 0.98 & 0.012 & 10.4 \\
\hline-156.460 & 20.755 & 12:08:00 & 27.88 & 8.22 & 46,344 & 30.32 & 17.0 & 188.5 & 12.56 & 764 & 0.87 & 0.011 & 10.4 \\
\hline-156.460 & 20.755 & $12: 08: 30$ & 27.82 & 8.23 & 46,782 & 30.64 & 33.9 & 188.8 & 12.57 & 764 & 1.02 & 0.013 & 10.4 \\
\hline-156.460 & 20.755 & 12:09:00 & 28.03 & 8.22 & 46,359 & 30.34 & 36.6 & 190.9 & 12.68 & 764 & 0.80 & 0.011 & 10.4 \\
\hline-156.460 & 20.755 & $12: 09: 30$ & 27.88 & 8.23 & 46,670 & 30.56 & 47.5 & 188.8 & 12.56 & 764 & 0.77 & 0.011 & 10.9 \\
\hline-156.460 & 20.754 & $12: 10: 00$ & 27.61 & 8.26 & 47,815 & 31.38 & 68.9 & 194.1 & 12.91 & 764 & 0.63 & 0.009 & 10.9 \\
\hline-156.460 & 20.754 & $12: 10: 30$ & 27.69 & 8.24 & 48,753 & 32.08 & 102.7 & 199.0 & 13.16 & 764 & 0.50 & 0.008 & 11.0 \\
\hline
\end{tabular}


Table A1. Nearshore wading survey with multiparameter water-quality sonde at Kihei, Hawaii, May 8, 2008-Continued.

[Abbreviations: ${ }^{\circ} \mathrm{C}$, degrees Celsius; $\mu \mathrm{S} / \mathrm{cm}$, microSiemens per centimeter; NTU, nephelometric turbidity units; mg/L, milligrams per liter; mm Hg, millimeters mercury; $\mu \mathrm{g} / \mathrm{L}$, micrograms per liter; salinity is dimensionless; chlorophyll values displaying an out-of-range flag were deleted]

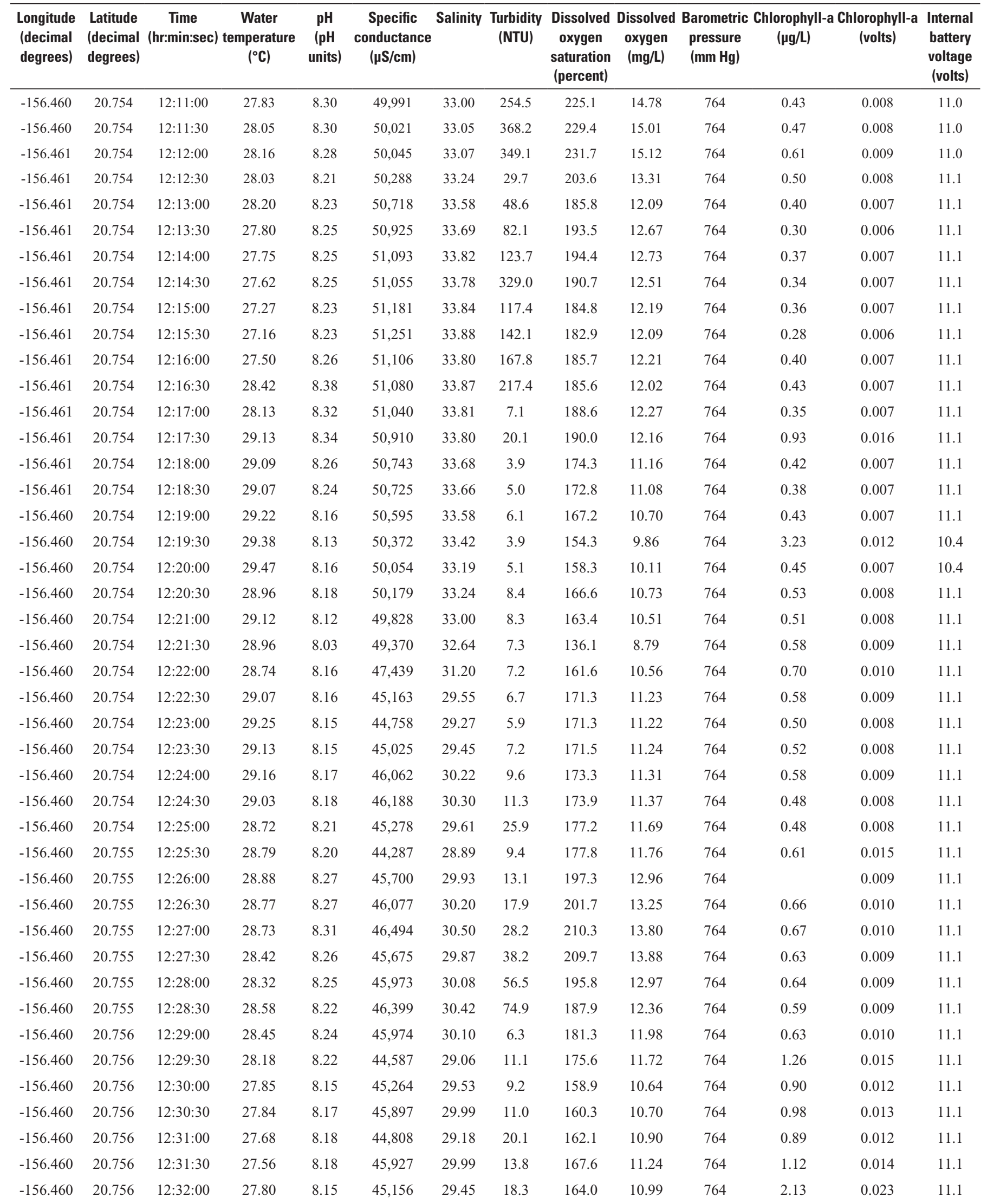


Table A1. Nearshore wading survey with multiparameter water-quality sonde at Kihei, Hawaii, May 8, 2008-Continued.

[Abbreviations: ${ }^{\circ} \mathrm{C}$, degrees Celsius; $\mu \mathrm{S} / \mathrm{cm}$, microSiemens per centimeter; $\mathrm{NTU}$, nephelometric turbidity units; $\mathrm{mg} / \mathrm{L}$, milligrams per liter; mm Hg, millimeters mercury; $\mu \mathrm{g} / \mathrm{L}$, micrograms per liter; salinity is dimensionless; chlorophyll values displaying an out-of-range flag were deleted]

\begin{tabular}{|c|c|c|c|c|c|c|c|c|c|c|c|c|c|}
\hline $\begin{array}{l}\text { Longitude } \\
\text { (decimal } \\
\text { degrees) }\end{array}$ & $\begin{array}{l}\text { Latitude } \\
\text { (decimal } \\
\text { degrees) }\end{array}$ & $\begin{array}{c}\text { Time } \\
\text { (hr:min:sec) }\end{array}$ & $\begin{array}{c}\text { Water } \\
\text { temperature } \\
\left({ }^{\circ} \mathrm{C}\right)\end{array}$ & $\begin{array}{c}\text { pH } \\
\text { (pH } \\
\text { units) }\end{array}$ & $\begin{array}{c}\text { Specific } \\
\text { conductance } \\
(\mu \mathrm{S} / \mathrm{cm})\end{array}$ & Salinity & $\begin{array}{c}\text { Turbidity } \\
\text { (NTU) }\end{array}$ & $\begin{array}{l}\text { Dissolved } \\
\text { oxygen } \\
\text { saturation } \\
\text { (percent) }\end{array}$ & $\begin{array}{c}\text { Dissolved } \\
\text { oxygen } \\
\text { (mg/L) }\end{array}$ & $\begin{array}{l}\text { Barometric } \\
\text { pressure } \\
(\mathrm{mm} \mathrm{Hg})\end{array}$ & $\begin{array}{c}\text { Chlorophyll-a } \\
\text { ( } \mu g / L)\end{array}$ & $\begin{array}{l}\text {-a Chlorophyll-a } \\
\text { (volts) }\end{array}$ & $\begin{array}{r}\text { Internal } \\
\text { battery } \\
\text { voltage } \\
\text { (volts) }\end{array}$ \\
\hline-156.460 & 20.757 & $12: 33: 00$ & 27.99 & 8.11 & 45,558 & 29.75 & 19.6 & 144.8 & 9.66 & 764 & & 0.012 & 11.1 \\
\hline-156.460 & 20.757 & $12: 33: 30$ & 28.00 & 8.10 & 45,724 & 29.88 & 15.5 & 148.4 & 9.89 & 764 & 0.89 & 0.012 & 11.1 \\
\hline-156.460 & 20.757 & $12: 34: 30$ & 28.04 & 8.07 & 46,542 & 30.48 & 34.1 & 136.4 & 9.05 & 764 & 0.92 & 0.012 & 11.1 \\
\hline-156.460 & 20.757 & $12: 35: 00$ & 28.28 & 8.07 & 46,729 & 30.64 & 12.9 & 140.3 & 9.27 & 764 & 0.96 & 0.013 & 11.1 \\
\hline-156.460 & 20.757 & $12: 35: 30$ & 28.55 & 8.06 & 47,175 & 30.99 & 14.4 & 134.7 & 8.84 & 764 & 0.98 & 0.012 & 11.1 \\
\hline-156.460 & 20.757 & $12: 36: 00$ & 28.33 & 8.09 & 47,698 & 31.35 & 13.4 & 133.3 & 8.76 & 764 & 1.26 & 0.010 & 11.1 \\
\hline-156.460 & 20.758 & $12: 36: 30$ & 28.25 & 8.08 & 48,010 & 31.58 & 11.4 & 136.2 & 8.96 & 764 & 0.83 & 0.011 & 11.1 \\
\hline-156.460 & 20.758 & $12: 38: 30$ & 27.27 & 8.15 & 48,307 & 31.71 & 5.6 & 155.0 & 10.35 & 764 & 0.49 & 0.008 & 11.1 \\
\hline-156.460 & 20.758 & $12: 39: 00$ & 27.21 & 8.15 & 48,643 & 31.95 & 3.7 & 162.7 & 10.86 & 764 & 0.45 & 0.008 & 11.1 \\
\hline-156.460 & 20.758 & $12: 39: 30$ & 27.42 & 8.12 & 48,106 & 31.57 & 4.0 & 158.5 & 10.57 & 764 & 0.53 & 0.008 & 11.1 \\
\hline-156.460 & 20.758 & $12: 40: 00$ & 28.45 & 8.04 & 48,341 & 31.84 & 11.4 & 136.8 & 8.95 & 764 & 0.66 & 0.010 & 11.1 \\
\hline-156.460 & 20.758 & $12: 40: 30$ & 28.25 & 8.07 & 48,334 & 31.82 & 10.3 & 136.5 & 8.96 & 764 & 0.67 & 0.010 & 11.1 \\
\hline-156.460 & 20.758 & $12: 41: 00$ & 28.52 & 8.05 & 48,500 & 31.96 & 9.8 & 127.1 & 8.30 & 764 & 0.63 & 0.009 & 11.1 \\
\hline-156.460 & 20.758 & $12: 41: 30$ & 28.24 & 8.03 & 48,804 & 32.16 & 9.5 & 124.1 & 8.14 & 764 & 0.66 & 0.010 & 11.1 \\
\hline-156.460 & 20.757 & $12: 42: 00$ & 27.91 & 8.09 & 48,367 & 31.81 & 8.1 & 140.9 & 9.30 & 764 & 0.61 & 0.009 & 11.1 \\
\hline-156.460 & 20.757 & $12: 42: 30$ & 27.84 & 8.09 & 48,185 & 31.67 & 9.4 & 146.5 & 9.69 & 764 & 0.67 & 0.010 & 11.1 \\
\hline-156.460 & 20.756 & $12: 46: 00$ & 27.61 & 8.16 & 46,815 & 30.64 & 15.1 & 161.3 & 10.77 & 764 & 0.55 & 0.009 & 11.1 \\
\hline-156.460 & 20.756 & $12: 46: 30$ & 27.60 & 8.17 & 46,822 & 30.65 & 26.0 & 163.8 & 10.94 & 764 & 0.58 & 0.009 & 11.1 \\
\hline-156.460 & 20.756 & $12: 47: 00$ & 27.61 & 8.17 & 46,984 & 30.77 & 32.9 & 167.9 & 11.20 & 764 & 0.64 & 0.009 & 11.1 \\
\hline-156.460 & 20.756 & $12: 47: 30$ & 27.57 & 8.19 & 47,360 & 31.04 & 49.9 & 171.9 & 11.46 & 764 & 0.61 & 0.009 & 11.1 \\
\hline-156.460 & 20.756 & $12: 48: 00$ & 27.57 & 8.20 & 47,401 & 31.07 & 26.2 & 179.4 & 11.96 & 764 & 0.60 & 0.009 & 11.1 \\
\hline-156.460 & 20.756 & $12: 48: 30$ & 27.60 & 8.22 & 47,308 & 31.00 & 33.4 & 183.1 & 12.21 & 764 & 0.63 & 0.009 & 11.2 \\
\hline-156.460 & 20.755 & $12: 49: 00$ & 27.63 & 8.21 & 47,399 & 31.07 & 24.9 & 185.1 & 12.33 & 764 & 0.56 & 0.008 & 11.2 \\
\hline-156.460 & 20.755 & $12: 49: 30$ & 27.77 & 8.24 & 46,992 & 30.79 & 34.1 & 187.9 & 12.51 & 764 & 0.56 & 0.009 & 11.1 \\
\hline
\end{tabular}


Table A2. Nearshore kayak survey with multiparameter water-quality sonde at Kihei, Hawaii, May 22, 2008.

[Abbreviations: ${ }^{\circ} \mathrm{C}$, degrees Celsius; $\mu \mathrm{S} / \mathrm{cm}$, microSiemens per centimeter; NTU, nephelometric turbidity units; $\mathrm{mg} / \mathrm{L}$, milligrams per liter; mm Hg, millimeters mercury; $\mu \mathrm{g} / \mathrm{L}$, micrograms per liter; salinity is dimensionless; deleted data include chlorophyll values displaying an out-of-range flag and the entire dissolved oxygen record, for which the calibration was in error]

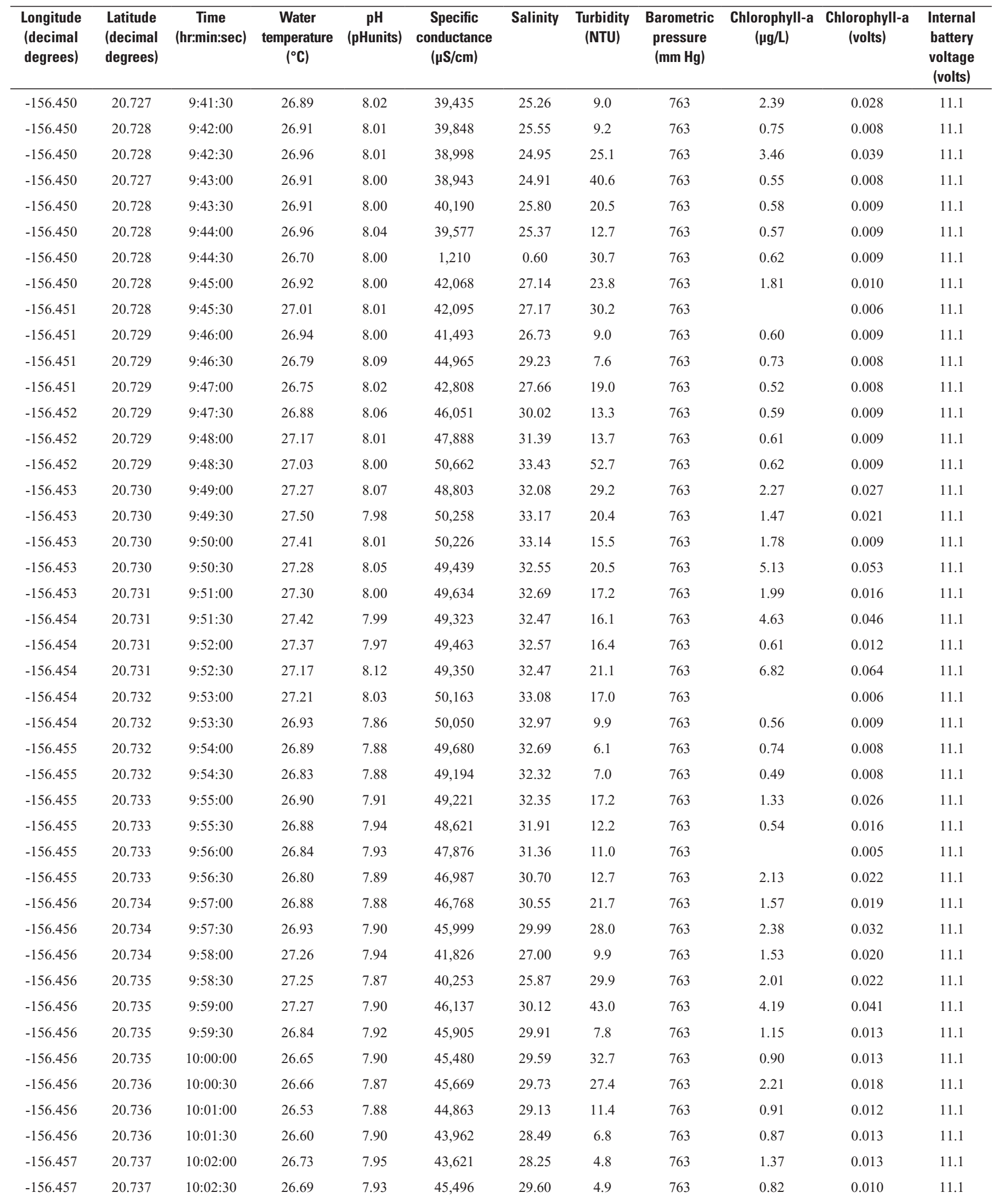


Table A2. Nearshore kayak survey with multiparameter water-quality sonde at Kihei, Hawaii, May 22, 2008-Continued.

[Abbreviations: ${ }^{\circ} \mathrm{C}$, degrees Celsius; $\mu \mathrm{S} / \mathrm{cm}$, microSiemens per centimeter; NTU, nephelometric turbidity units; $\mathrm{mg} / \mathrm{L}$, milligrams per liter; mm Hg, millimeters mercury; $\mu \mathrm{g} / \mathrm{L}$, micrograms per liter; salinity is dimensionless; deleted data include chlorophyll values displaying an out-of-range flag and the entire dissolved oxygen record, for which the calibration was in error]

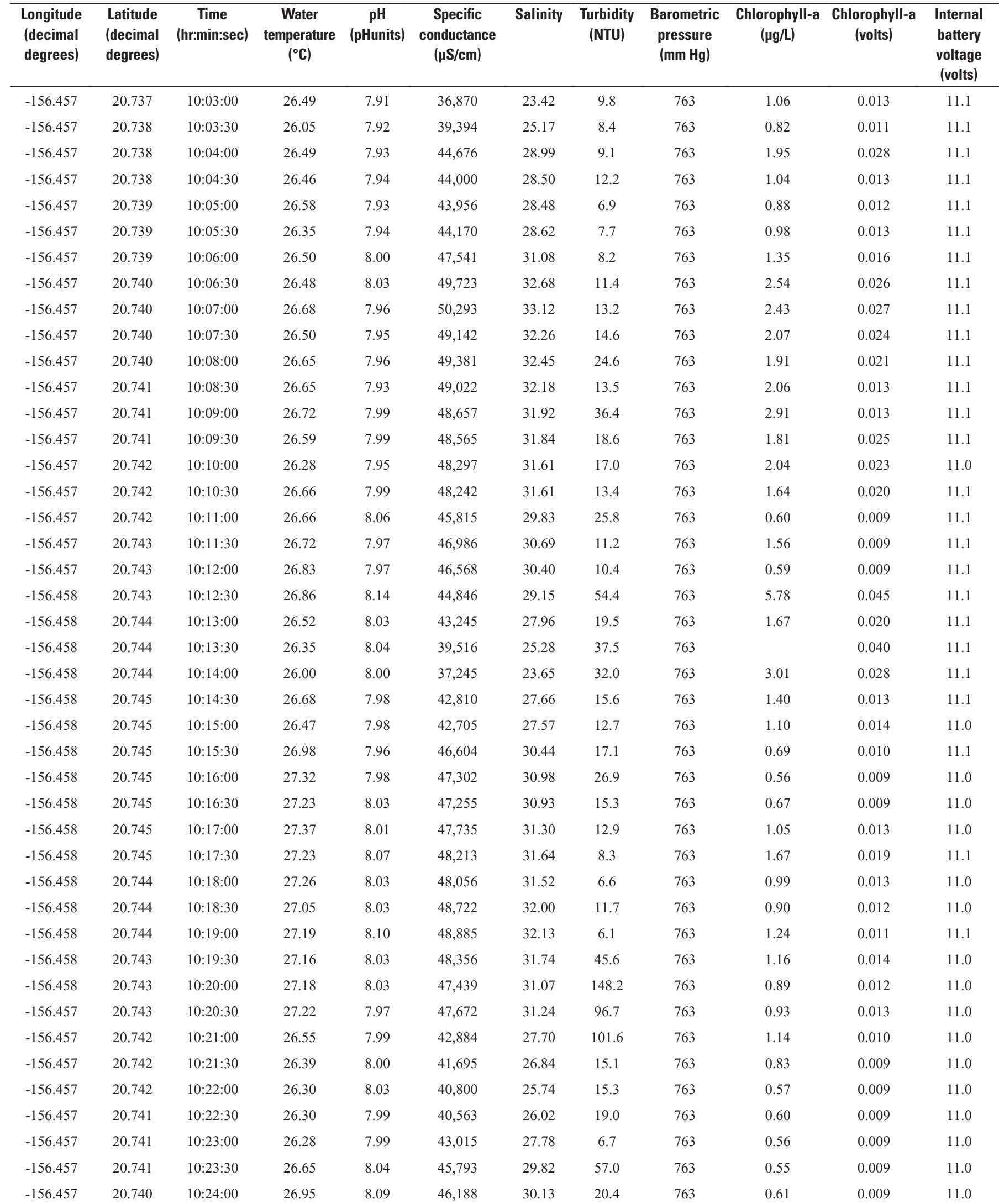


Table A2. Nearshore kayak survey with multiparameter water-quality sonde at Kihei, Hawaii, May 22, 2008-Continued.

[Abbreviations: ${ }^{\circ} \mathrm{C}$, degrees Celsius; $\mu \mathrm{S} / \mathrm{cm}$, microSiemens per centimeter; NTU, nephelometric turbidity units; $\mathrm{mg} / \mathrm{L}$, milligrams per liter; mm Hg, millimeters mercury; $\mu \mathrm{g} / \mathrm{L}$, micrograms per liter; salinity is dimensionless; deleted data include chlorophyll values displaying an out-of-range flag and the entire dissolved oxygen record, for which the calibration was in error]

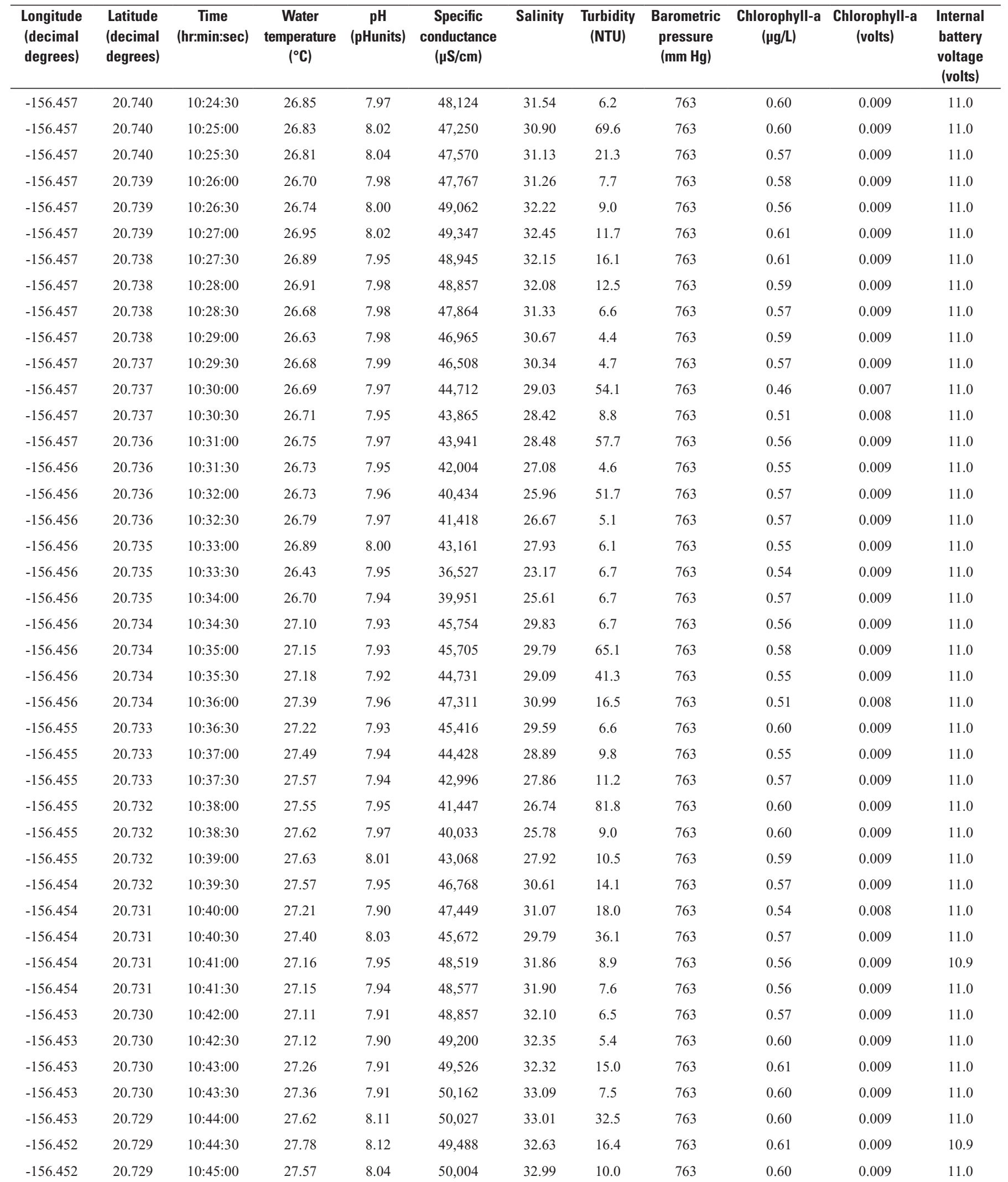


Table A2. Nearshore kayak survey with multiparameter water-quality sonde at Kihei, Hawaii, May 22, 2008-Continued.

[Abbreviations: ${ }^{\circ} \mathrm{C}$, degrees Celsius; $\mu \mathrm{S} / \mathrm{cm}$, microSiemens per centimeter; NTU, nephelometric turbidity units; mg/L, milligrams per liter; mm Hg, millimeters mercury; $\mu \mathrm{g} / \mathrm{L}$, micrograms per liter; salinity is dimensionless; deleted data include chlorophyll values displaying an out-of-range flag and the entire dissolved oxygen record, for which the calibration was in error]

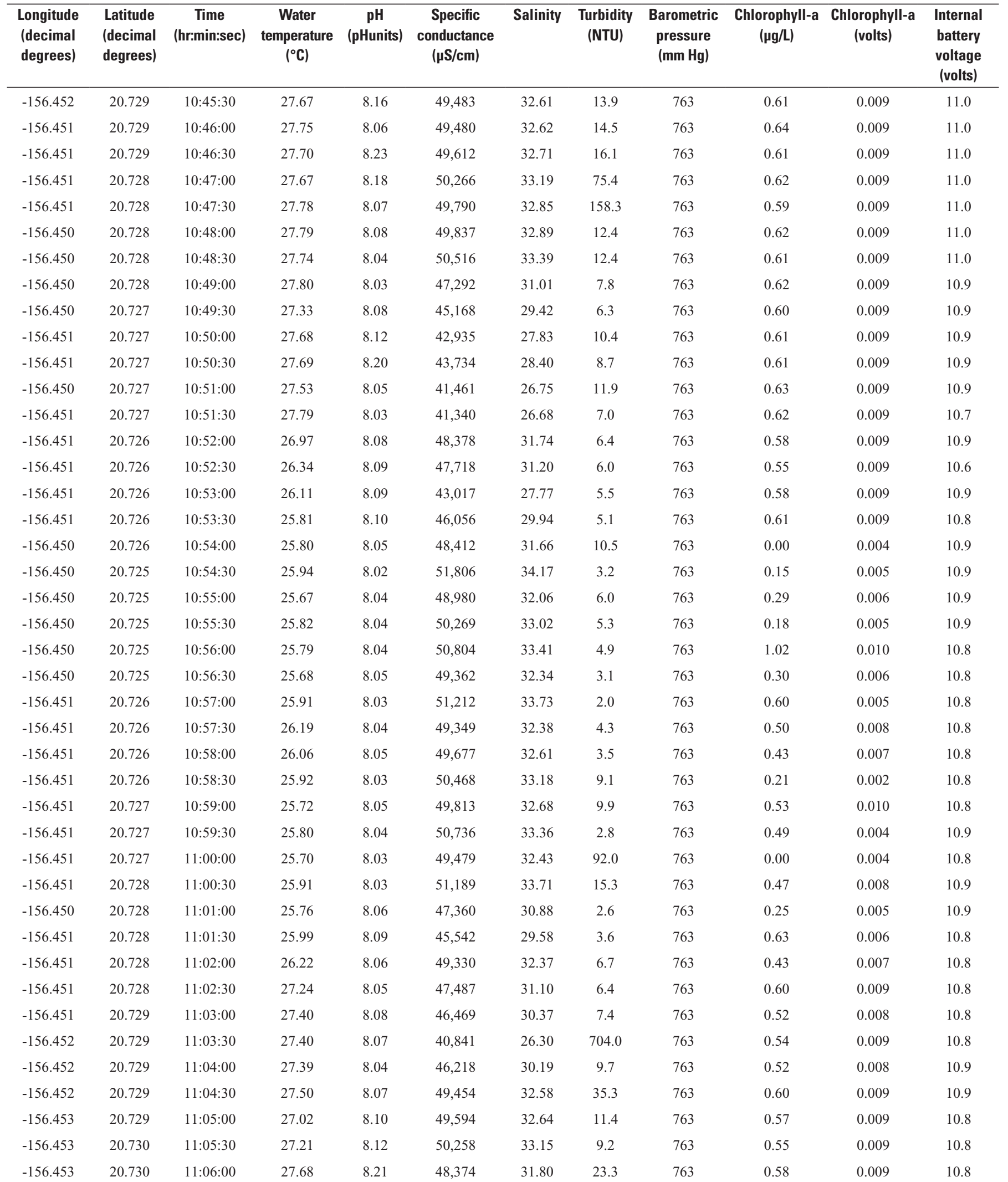


Table A2. Nearshore kayak survey with multiparameter water-quality sonde at Kihei, Hawaii, May 22, 2008-Continued.

[Abbreviations: ${ }^{\circ} \mathrm{C}$, degrees Celsius; $\mu \mathrm{S} / \mathrm{cm}$, microSiemens per centimeter; NTU, nephelometric turbidity units; $\mathrm{mg} / \mathrm{L}$, milligrams per liter; mm Hg, millimeters mercury; $\mu \mathrm{g} / \mathrm{L}$, micrograms per liter; salinity is dimensionless; deleted data include chlorophyll values displaying an out-of-range flag and the entire dissolved oxygen record, for which the calibration was in error]

\begin{tabular}{|c|c|c|c|c|c|c|c|c|c|c|c|}
\hline $\begin{array}{l}\text { Longitude } \\
\text { (decimal } \\
\text { degrees) }\end{array}$ & $\begin{array}{l}\text { Latitude } \\
\text { (decimal } \\
\text { degrees) }\end{array}$ & $\begin{array}{c}\text { Time } \\
\text { (hr:min:sec) }\end{array}$ & $\begin{array}{c}\text { Water } \\
\text { temperature } \\
\left({ }^{\circ} \mathrm{C}\right)\end{array}$ & $\begin{array}{c}\mathrm{pH} \\
\text { (pHunits) }\end{array}$ & $\begin{array}{c}\text { Specific } \\
\text { conductance } \\
(\mu \mathrm{S} / \mathrm{cm})\end{array}$ & Salinity & $\begin{array}{l}\text { Turbidity } \\
\text { (NTU) }\end{array}$ & $\begin{array}{c}\text { Barometric } \\
\text { pressure } \\
(\mathrm{mm} \mathrm{Hg})\end{array}$ & $\begin{array}{c}\text { Chlorophyll-a } \\
(\mu \mathrm{g} / \mathrm{L})\end{array}$ & $\begin{array}{l}\text { Chlorophyll-a } \\
\text { (volts) }\end{array}$ & $\begin{array}{c}\text { Internal } \\
\text { battery } \\
\text { voltage } \\
\text { (volts) }\end{array}$ \\
\hline-156.454 & 20.730 & 11:07:00 & 27.78 & 8.18 & 50,173 & 33.13 & 10.5 & 763 & 0.54 & 0.008 & 10.8 \\
\hline-156.454 & 20.731 & 11:07:30 & 27.81 & 8.03 & 50,098 & 33.08 & 7.7 & 763 & 0.55 & 0.009 & 10.8 \\
\hline-156.454 & 20.732 & 11:09:00 & 27.72 & 7.99 & 49,878 & 32.91 & 129.0 & 763 & 0.53 & 0.008 & 10.9 \\
\hline-156.455 & 20.732 & 11:09:30 & 27.76 & 7.95 & 51,061 & 33.79 & 8.5 & 763 & 0.59 & 0.009 & 10.8 \\
\hline-156.455 & 20.732 & 11:10:00 & 27.74 & 7.93 & 50,637 & 33.48 & 88.7 & 763 & 0.57 & 0.009 & 10.8 \\
\hline-156.455 & 20.732 & $11: 10: 30$ & 27.58 & 7.98 & 49,555 & 32.66 & 10.3 & 763 & 0.53 & 0.008 & 10.8 \\
\hline-156.456 & 20.734 & $11: 12: 30$ & 27.55 & 7.99 & 49,365 & 32.52 & 180.7 & 763 & 0.37 & 0.007 & 10.8 \\
\hline-156.456 & 20.734 & 11:13:00 & 27.49 & 7.92 & 49,256 & 32.43 & 5.7 & 763 & 0.47 & 0.006 & 10.8 \\
\hline-156.456 & 20.734 & $11: 13: 30$ & 27.52 & 8.02 & 47,551 & 31.18 & 22.0 & 763 & 0.56 & 0.009 & 10.8 \\
\hline-156.456 & 20.735 & 11:14:00 & 27.81 & 8.04 & 45,505 & 29.70 & 7.5 & 763 & 0.53 & 0.008 & 10.8 \\
\hline-156.456 & 20.735 & 11:14:30 & 27.71 & 8.02 & 46,111 & 30.14 & 8.3 & 763 & 0.53 & 0.008 & 10.8 \\
\hline-156.456 & 20.735 & 11:15:00 & 27.59 & 8.00 & 47,461 & 31.12 & 5.3 & 763 & 0.51 & 0.008 & 10.8 \\
\hline-156.457 & 20.736 & $11: 15: 30$ & 27.69 & 7.99 & 46,851 & 30.68 & 9.8 & 763 & 0.50 & 0.008 & 10.8 \\
\hline-156.457 & 20.736 & 11:16:00 & 27.68 & 7.98 & 47,088 & 30.85 & 6.9 & 763 & 0.45 & 0.007 & 10.8 \\
\hline-156.457 & 20.736 & $11: 16: 30$ & 27.72 & 7.98 & 46,946 & 30.75 & 5.3 & 763 & 0.40 & 0.007 & 10.8 \\
\hline-156.457 & 20.739 & 11:20:00 & 27.31 & 7.96 & 45,087 & 29.36 & 4.8 & 763 & 0.55 & 0.009 & 10.8 \\
\hline-156.457 & 20.739 & $11: 20: 30$ & 27.35 & 7.97 & 46,496 & 30.39 & 4.8 & 763 & 0.33 & 0.007 & 10.8 \\
\hline-156.457 & 20.740 & $11: 21: 00$ & 27.32 & 7.97 & 45,088 & 29.92 & 4.6 & 763 & 0.33 & 0.007 & 10.8 \\
\hline-156.457 & 20.740 & $11: 21: 30$ & 27.28 & 7.99 & 45,851 & 29.91 & 16.6 & 763 & 0.36 & 0.006 & 10.8 \\
\hline-156.457 & 20.740 & $11: 22: 00$ & 27.26 & 8.00 & 46,237 & 30.19 & 3.8 & 763 & 0.51 & 0.008 & 10.8 \\
\hline-156.457 & 20.741 & $11: 22: 30$ & 27.28 & 7.99 & 45,969 & 30.00 & 4.7 & 763 & 0.49 & 0.008 & 10.8 \\
\hline-156.457 & 20.741 & $11: 23: 00$ & 27.29 & 8.02 & 46,321 & 30.26 & 4.0 & 763 & 0.57 & 0.009 & 10.8 \\
\hline-156.457 & 20.741 & $11: 23: 30$ & 27.26 & 8.01 & 45,519 & 29.67 & 8.6 & 763 & 0.57 & 0.009 & 10.8 \\
\hline-156.457 & 20.742 & $11: 24: 00$ & 27.27 & 8.01 & 47,479 & 31.10 & 29.1 & 763 & 0.56 & 0.009 & 10.8 \\
\hline-156.457 & 20.742 & $11: 24: 30$ & 27.32 & 8.02 & 48,356 & 31.75 & 4.0 & 763 & 0.61 & 0.009 & 10.8 \\
\hline-156.458 & 20.742 & $11: 25: 00$ & 27.38 & 8.02 & 48,911 & 32.16 & 5.5 & 763 & 0.58 & 0.009 & 10.8 \\
\hline-156.458 & 20.743 & $11: 25: 30$ & 27.65 & 8.10 & 49,833 & 32.87 & 61.4 & 763 & 0.58 & 0.009 & 10.8 \\
\hline-156.458 & 20.743 & 11:26:00 & 27.82 & 8.18 & 50,076 & 33.07 & 7.2 & 763 & 0.63 & 0.009 & 10.8 \\
\hline-156.458 & 20.743 & $11: 26: 30$ & 27.82 & 8.20 & 49,252 & 32.46 & 15.4 & 763 & 0.60 & 0.009 & 10.8 \\
\hline-156.458 & 20.744 & $11: 27: 00$ & 27.91 & 8.14 & 49,740 & 32.83 & 6.4 & 763 & 0.61 & 0.009 & 10.8 \\
\hline-156.458 & 20.744 & $11: 27: 30$ & 28.12 & 8.04 & 50,049 & 33.07 & 8.4 & 763 & 0.62 & 0.009 & 10.8 \\
\hline
\end{tabular}


Table A2. Nearshore kayak survey with multiparameter water-quality sonde at Kihei, Hawaii, May 22, 2008-Continued.

[Abbreviations: ${ }^{\circ} \mathrm{C}$, degrees Celsius; $\mu \mathrm{S} / \mathrm{cm}$, microSiemens per centimeter; NTU, nephelometric turbidity units; $\mathrm{mg} / \mathrm{L}$, milligrams per liter; mm Hg, millimeters mercury; $\mu \mathrm{g} / \mathrm{L}$, micrograms per liter; salinity is dimensionless; deleted data include chlorophyll values displaying an out-of-range flag and the entire dissolved oxygen record, for which the calibration was in error]

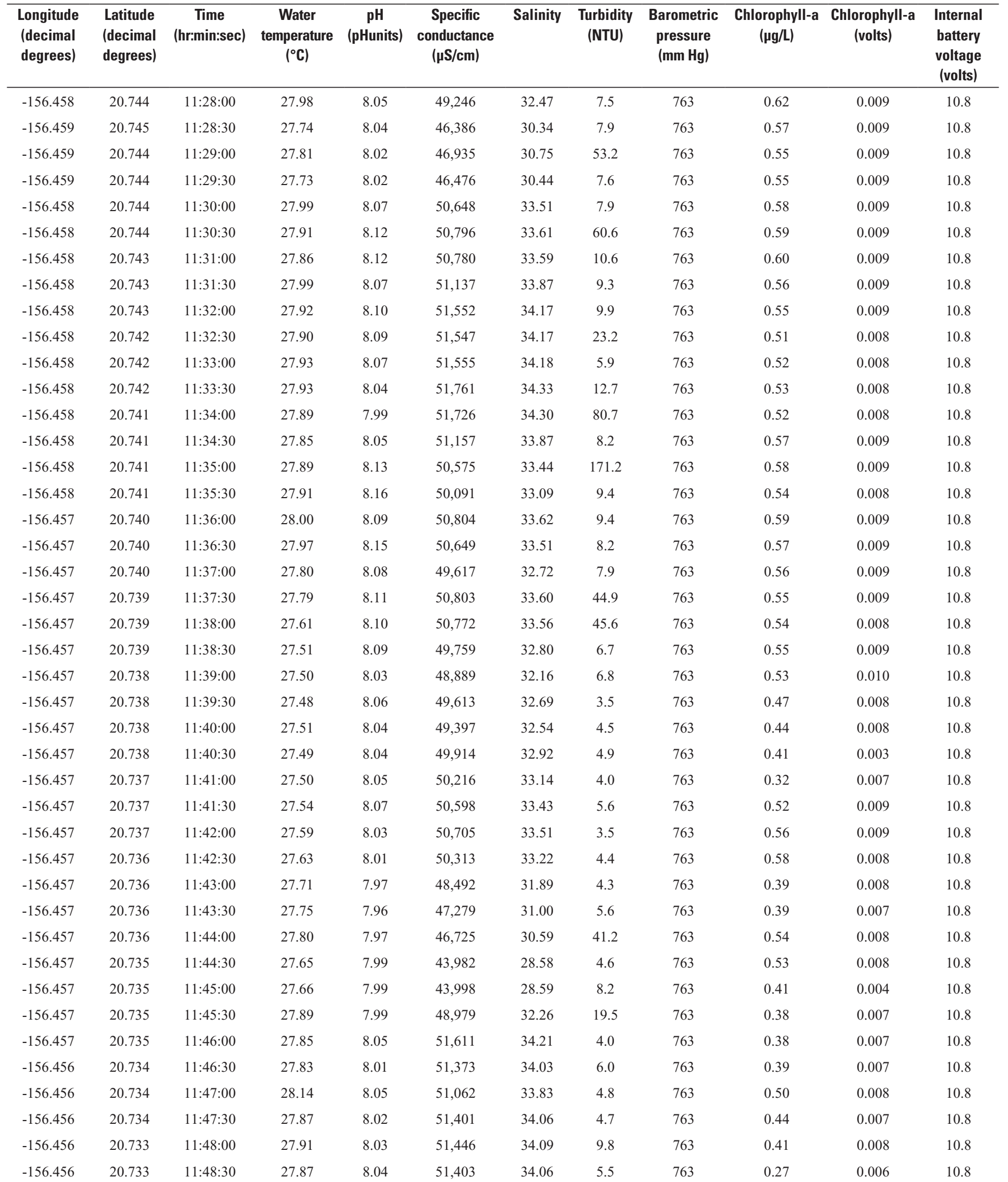


Table A2. Nearshore kayak survey with multiparameter water-quality sonde at Kihei, Hawaii, May 22, 2008-Continued.

[Abbreviations: ${ }^{\circ} \mathrm{C}$, degrees Celsius; $\mu \mathrm{S} / \mathrm{cm}$, microSiemens per centimeter; NTU, nephelometric turbidity units; $\mathrm{mg} / \mathrm{L}$, milligrams per liter; mm Hg, millimeters mercury; $\mu \mathrm{g} / \mathrm{L}$, micrograms per liter; salinity is dimensionless; deleted data include chlorophyll values displaying an out-of-range flag and the entire dissolved oxygen record, for which the calibration was in error]

\begin{tabular}{|c|c|c|c|c|c|c|c|c|c|c|c|}
\hline $\begin{array}{l}\text { Longitude } \\
\text { (decimal } \\
\text { degrees) }\end{array}$ & $\begin{array}{l}\text { Latitude } \\
\text { (decimal } \\
\text { degrees) }\end{array}$ & $\begin{array}{c}\text { Time } \\
\text { (hr:min:sec) }\end{array}$ & $\begin{array}{c}\text { Water } \\
\text { temperature } \\
\left({ }^{\circ} \mathrm{C}\right)\end{array}$ & $\begin{array}{c}\mathrm{pH} \\
\text { (pHunits) }\end{array}$ & $\begin{array}{c}\text { Specific } \\
\text { conductance } \\
\text { ( } \mathrm{S} / \mathrm{cm})\end{array}$ & Salinity & $\begin{array}{c}\text { Turbidity } \\
\text { (NTU) }\end{array}$ & $\begin{array}{c}\text { Barometric } \\
\text { pressure } \\
(\mathrm{mm} \mathrm{Hg})\end{array}$ & $\begin{array}{c}\text { Chlorophyll-a } \\
(\mu \mathrm{g} / \mathrm{L})\end{array}$ & $\begin{array}{l}\text { Chlorophyll-a } \\
\text { (volts) }\end{array}$ & $\begin{array}{c}\text { Internal } \\
\text { battery } \\
\text { voltage } \\
\text { (volts) }\end{array}$ \\
\hline-156.456 & 20.733 & 11:49:00 & 27.73 & 8.04 & 51,342 & 34.00 & 14.6 & 763 & 0.38 & 0.008 & 10.8 \\
\hline-156.456 & 20.733 & $11: 49: 30$ & 27.93 & 8.10 & 50,715 & 33.55 & 4.3 & 763 & 0.54 & 0.008 & 10.8 \\
\hline-156.456 & 20.732 & $11: 50: 00$ & 27.95 & 8.11 & 50,344 & 33.28 & 36.2 & 763 & 0.53 & 0.008 & 10.8 \\
\hline-156.455 & 20.732 & $11: 50: 30$ & 27.97 & 8.11 & 50,892 & 33.69 & 46.6 & 763 & 0.53 & 0.008 & 10.8 \\
\hline-156.455 & 20.732 & $11: 51: 00$ & 27.92 & 8.15 & 50,866 & 33.66 & 4.9 & 763 & 0.53 & 0.008 & 10.8 \\
\hline-156.455 & 20.732 & $11: 51: 30$ & 27.97 & 8.20 & 51,112 & 33.85 & 7.8 & 763 & 0.52 & 0.008 & 10.8 \\
\hline-156.455 & 20.731 & $11: 52: 00$ & 27.72 & 8.16 & 51,170 & 33.87 & 13.6 & 763 & 0.52 & 0.008 & 10.8 \\
\hline-156.455 & 20.731 & $11: 52: 30$ & 27.76 & 8.10 & 50,951 & 33.71 & 6.2 & 763 & 0.51 & 0.008 & 10.8 \\
\hline-156.454 & 20.731 & $11: 53: 00$ & 27.63 & 8.05 & 51,153 & 33.85 & 6.6 & 763 & 0.54 & 0.008 & 10.8 \\
\hline-156.454 & 20.731 & $11: 53: 30$ & 27.63 & 8.08 & 51,092 & 33.80 & 16.1 & 763 & 0.52 & 0.008 & 10.8 \\
\hline-156.454 & 20.730 & $11: 54: 00$ & 27.68 & 8.06 & 51,079 & 33.80 & 7.4 & 763 & 0.55 & 0.009 & 10.8 \\
\hline-156.454 & 20.730 & $11: 54: 30$ & 27.77 & 8.02 & 49,299 & 32.49 & 45.2 & 763 & 0.60 & 0.009 & 10.8 \\
\hline-156.454 & 20.730 & $11: 55: 00$ & 27.92 & 8.01 & 50,320 & 33.26 & 7.5 & 763 & 0.56 & 0.009 & 10.8 \\
\hline-156.453 & 20.730 & $11: 55: 30$ & 28.13 & 8.07 & 50,552 & 33.45 & 227.4 & 763 & 0.60 & 0.009 & 10.8 \\
\hline-156.453 & 20.729 & $11: 56: 00$ & 27.86 & 7.98 & 33,069 & 20.84 & 101.0 & 763 & 0.61 & 0.009 & 10.8 \\
\hline-156.453 & 20.729 & $11: 56: 30$ & 28.14 & 8.16 & 50,951 & 33.75 & 129.8 & 763 & 0.62 & 0.009 & 10.8 \\
\hline-156.453 & 20.729 & $11: 57: 00$ & 28.10 & 8.18 & 50,233 & 33.21 & 7.2 & 763 & 0.60 & 0.009 & 10.8 \\
\hline-156.452 & 20.729 & $11: 57: 30$ & 28.27 & 8.10 & 50,363 & 33.32 & 14.8 & 763 & 0.55 & 0.009 & 10.8 \\
\hline-156.452 & 20.729 & $11: 58: 00$ & 28.04 & 8.16 & 50,946 & 33.73 & 15.7 & 763 & 0.60 & 0.009 & 10.8 \\
\hline-156.452 & 20.728 & $11: 58: 30$ & 28.11 & 8.30 & 50,441 & 33.36 & 14.9 & 763 & 0.61 & 0.009 & 10.8 \\
\hline-156.451 & 20.728 & $11: 59: 00$ & 28.04 & 8.34 & 50,637 & 33.50 & 9.4 & 763 & 0.62 & 0.009 & 10.8 \\
\hline-156.451 & 20.728 & $11: 59: 30$ & 28.01 & 8.25 & 2,326 & 1.20 & 70.1 & 763 & 0.62 & 0.009 & 10.8 \\
\hline-156.451 & 20.728 & $12: 00: 00$ & 27.87 & 8.26 & 50,389 & 33.30 & 14.3 & 763 & 0.62 & 0.009 & 10.8 \\
\hline-156.451 & 20.728 & $12: 00: 30$ & 27.27 & 8.15 & 47,640 & 31.22 & 51.5 & 763 & 0.61 & 0.009 & 10.8 \\
\hline-156.451 & 20.728 & $12: 01: 00$ & 27.16 & 8.10 & 49,066 & 32.26 & 8.7 & 763 & 0.65 & 0.009 & 10.8 \\
\hline-156.450 & 20.727 & $12: 01: 30$ & 27.37 & 8.13 & 48,728 & 32.03 & 7.6 & 763 & 0.62 & 0.009 & 10.8 \\
\hline-156.450 & 20.727 & $12: 02: 00$ & 27.36 & 8.10 & 48,562 & 31.91 & 7.7 & 763 & 0.61 & 0.009 & 10.8 \\
\hline-156.450 & 20.727 & $12: 02: 30$ & 27.62 & 8.14 & 47,603 & 31.22 & 13.1 & 763 & 0.61 & 0.009 & 10.8 \\
\hline-156.450 & 20.727 & $12: 03: 00$ & 27.97 & 8.15 & 45,758 & 29.90 & 7.6 & 763 & 0.62 & 0.009 & 10.7 \\
\hline-156.450 & 20.728 & $12: 03: 30$ & 27.77 & 8.10 & 46,626 & 30.52 & 4.5 & 763 & 0.57 & 0.009 & 10.8 \\
\hline
\end{tabular}


Table A3. Nearshore wading survey with multiparameter water-quality sonde at Lahaina, Hawaii, May 6, 2008.

[Abbreviations: ${ }^{\circ} \mathrm{C}$, degrees Celsius; $\mu \mathrm{S} / \mathrm{cm}$, microSiemens per centimeter; NTU, nephelometric turbidity units; $\mathrm{mg} / \mathrm{L}$, milligrams per liter; $\mathrm{mm}$ Hg, millimeters mercury; $\mu \mathrm{g} / \mathrm{L}$, micrograms per liter; salinity is dimensionless; the entire dissolved oxygen record was deleted because the calibration was in error]

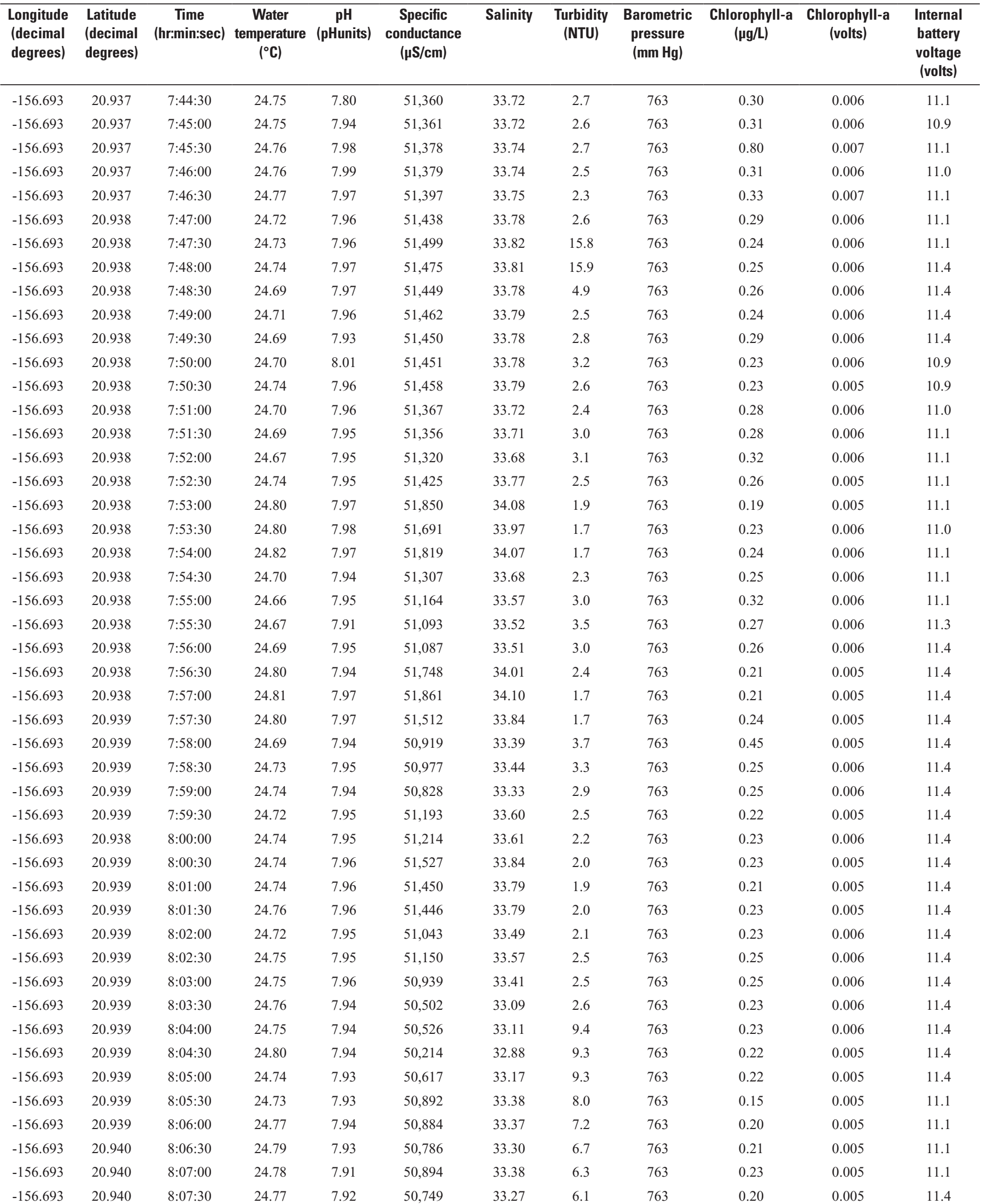


Table A3. Nearshore wading survey with multiparameter water-quality sonde at Lahaina, Hawaii, May 6, 2008-Continued.

[Abbreviations: ${ }^{\circ} \mathrm{C}$, degrees Celsius; $\mu \mathrm{S} / \mathrm{cm}$, microSiemens per centimeter; NTU, nephelometric turbidity units; mg/L, milligrams per liter; mm Hg, millimeters mercury; $\mu \mathrm{g} / \mathrm{L}$, micrograms per liter; salinity is dimensionless; the entire dissolved oxygen record was deleted because the calibration was in error]

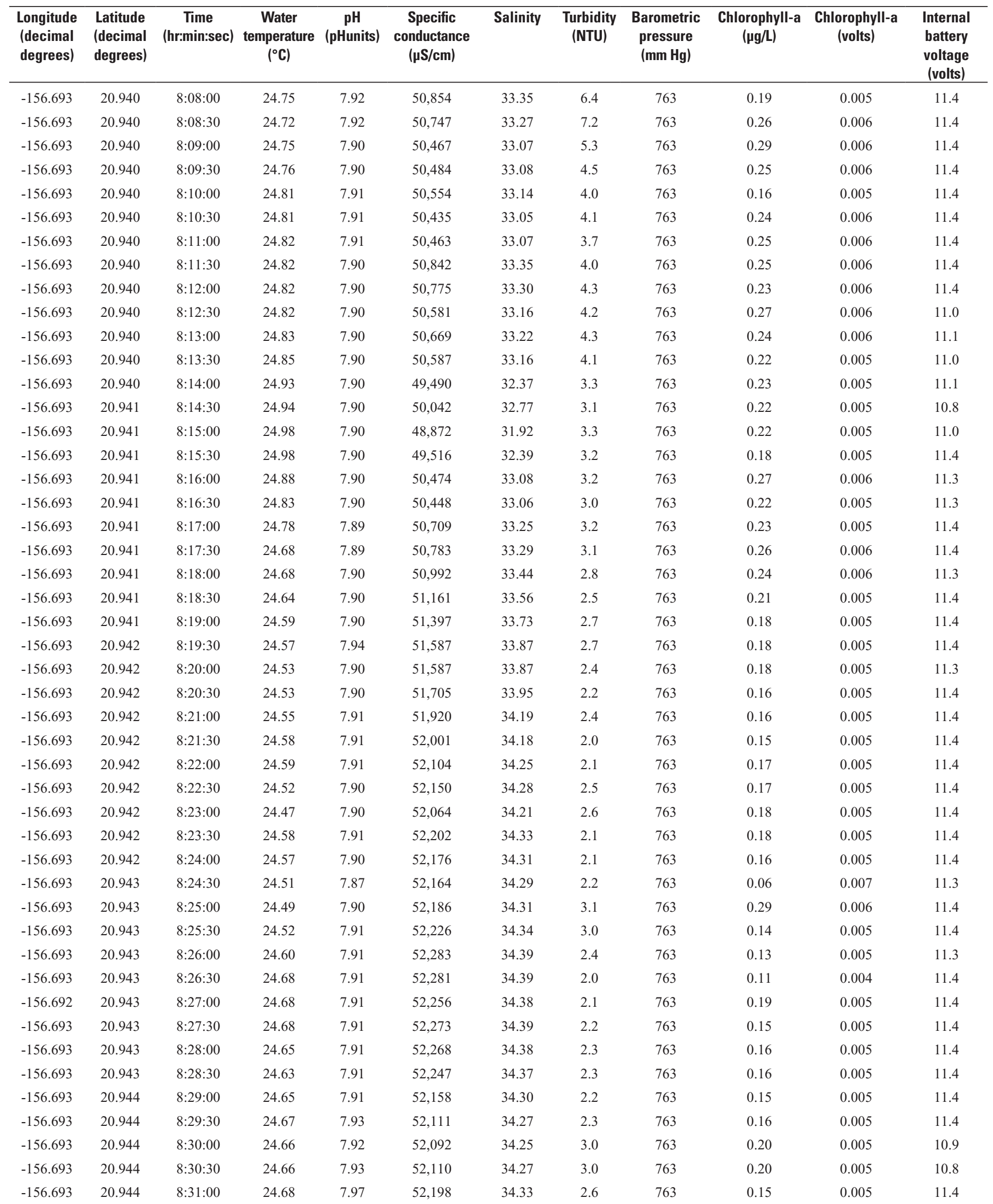


Table A3. Nearshore wading survey with multiparameter water-quality sonde at Lahaina, Hawaii, May 6, 2008_ Continued.

[Abbreviations: ${ }^{\circ} \mathrm{C}$, degrees Celsius; $\mu \mathrm{S} / \mathrm{cm}$, microSiemens per centimeter; NTU, nephelometric turbidity units; $\mathrm{mg} / \mathrm{L}$, milligrams per liter; mm Hg, millimeters mercury; $\mu \mathrm{g} / \mathrm{L}$, micrograms per liter; salinity is dimensionless; the entire dissolved oxygen record was deleted because the calibration was in error]

\begin{tabular}{|c|c|c|c|c|c|c|c|c|c|c|c|}
\hline $\begin{array}{l}\text { Longitude } \\
\text { (decimal } \\
\text { degrees) }\end{array}$ & $\begin{array}{l}\text { Latitude } \\
\text { (decimal } \\
\text { degrees) }\end{array}$ & $\begin{array}{c}\text { Time } \\
\text { (hr:min:sec) }\end{array}$ & $\begin{array}{c}\text { Water } \\
\text { temperature } \\
\left({ }^{\circ} \mathrm{C}\right)\end{array}$ & $\begin{array}{c}\mathrm{pH} \\
\text { (pHunits) }\end{array}$ & $\begin{array}{c}\text { Specific } \\
\text { conductance } \\
\text { ( } \mathrm{SS} / \mathrm{cm})\end{array}$ & Salinity & $\begin{array}{l}\text { Turbidity } \\
\text { (NTU) }\end{array}$ & $\begin{array}{c}\text { Barometric } \\
\text { pressure } \\
(\mathrm{mm} \mathrm{Hg})\end{array}$ & $\begin{array}{c}\text { Chlorophyll-a } \\
\text { ( } \mu \mathrm{g} / \mathrm{L})\end{array}$ & $\begin{array}{l}\text { Chlorophyll-a } \\
\text { (volts) }\end{array}$ & $\begin{array}{c}\text { Internal } \\
\text { battery } \\
\text { voltage } \\
\text { (volts) } \\
\end{array}$ \\
\hline-156.693 & 20.944 & 8:32:00 & 24.83 & 7.94 & 52,353 & 34.46 & 1.1 & 763 & 0.11 & 0.004 & 11.4 \\
\hline-156.693 & 20.944 & $8: 32: 30$ & 24.84 & 7.94 & 52,354 & 34.47 & 1.0 & 763 & 0.08 & 0.004 & 11.4 \\
\hline-156.693 & 20.944 & 8:33:00 & 24.82 & 7.95 & 52,317 & 34.44 & 1.0 & 763 & 0.12 & 0.004 & 11.4 \\
\hline-156.693 & 20.944 & $8: 34: 00$ & 24.76 & 7.95 & 52,229 & 34.36 & 1.6 & 763 & 0.12 & 0.005 & 11.4 \\
\hline-156.693 & 20.944 & $8: 34: 30$ & 24.84 & 7.95 & 52,347 & 34.46 & 1.4 & 763 & 0.12 & 0.005 & 11.4 \\
\hline-156.693 & 20.944 & 8:35:00 & 24.86 & 7.96 & 52,392 & 34.50 & 1.1 & 763 & 0.14 & 0.005 & 11.3 \\
\hline-156.693 & 20.944 & $8: 35: 30$ & 24.81 & 7.93 & 52,299 & 34.42 & 1.1 & 763 & 0.16 & 0.005 & 11.4 \\
\hline-156.693 & 20.944 & $8: 36: 00$ & 24.82 & 7.95 & 52,285 & 34.41 & 1.3 & 763 & 0.11 & 0.004 & 11.4 \\
\hline-156.693 & 20.945 & 8:38:00 & 24.85 & 7.96 & 52,391 & 34.49 & 1.1 & 763 & 0.11 & 0.004 & 11.4 \\
\hline-156.693 & 20.945 & $8: 38: 30$ & 24.85 & 7.97 & 52,417 & 34.51 & 0.9 & 763 & 0.13 & 0.005 & 11.4 \\
\hline-156.693 & 20.945 & 8:39:00 & 24.86 & 7.96 & 52,410 & 34.51 & 0.9 & 763 & 0.14 & 0.005 & 11.4 \\
\hline-156.693 & 20.945 & $8: 39: 30$ & 24.86 & 7.96 & 52,392 & 34.50 & 0.9 & 763 & 0.14 & 0.005 & 11.4 \\
\hline-156.693 & 20.945 & 8:40:00 & 24.85 & 7.96 & 52,375 & 34.48 & 1.0 & 763 & 0.14 & 0.005 & 11.4 \\
\hline-156.693 & 20.945 & $8: 40: 30$ & 24.84 & 7.94 & 52,380 & 34.49 & 1.0 & 763 & 0.15 & 0.005 & 11.4 \\
\hline-156.693 & 20.946 & 8:41:00 & 24.84 & 7.97 & 52,363 & 34.47 & 1.1 & 763 & 0.16 & 0.005 & 11.4 \\
\hline-156.693 & 20.946 & $8: 41: 30$ & 24.84 & 7.96 & 52,355 & 34.47 & 1.1 & 763 & 0.15 & 0.005 & 11.4 \\
\hline-156.693 & 20.946 & 8:42:00 & 24.84 & 7.93 & 52,381 & 34.49 & 1.2 & 763 & 0.15 & 0.005 & 11.4 \\
\hline-156.693 & 20.946 & $8: 42: 30$ & 24.85 & 7.95 & 52,323 & 34.44 & 1.1 & 763 & 0.13 & 0.005 & 11.4 \\
\hline-156.693 & 20.946 & $8: 46: 30$ & 24.89 & 7.95 & 52,382 & 34.49 & 1.1 & 763 & 0.13 & 0.005 & 11.0 \\
\hline-156.693 & 20.946 & $8: 47: 00$ & 24.89 & 7.97 & 52,407 & 34.51 & 1.1 & 763 & 0.11 & 0.004 & 11.0 \\
\hline-156.693 & 20.946 & $8: 47: 30$ & 24.88 & 7.97 & 52,406 & 34.51 & 1.0 & 763 & 0.08 & 0.004 & 11.1 \\
\hline-156.693 & 20.946 & 8:48:00 & 24.87 & 7.97 & 52,414 & 34.51 & 0.9 & 763 & 0.09 & 0.004 & 11.4 \\
\hline-156.693 & 20.945 & 8:48:30 & 24.89 & 7.96 & 52,407 & 34.51 & 0.9 & 763 & 0.09 & 0.004 & 11.4 \\
\hline-156.693 & 20.945 & 8:49:00 & 24.88 & 7.96 & 52,413 & 34.51 & 1.0 & 763 & 0.11 & 0.004 & 11.4 \\
\hline-156.693 & 20.945 & $8: 49: 30$ & 24.85 & 7.95 & 52,348 & 34.46 & 1.1 & 763 & 0.14 & 0.005 & 11.4 \\
\hline-156.693 & 20.945 & 8:50:00 & 24.81 & 7.95 & 52,349 & 34.46 & 1.2 & 763 & 0.13 & 0.005 & 11.1 \\
\hline-156.693 & 20.945 & $8: 50: 30$ & 24.89 & 7.95 & 52,373 & 34.48 & 1.2 & 763 & 0.13 & 0.005 & 11.1 \\
\hline-156.693 & 20.945 & 8:51:00 & 24.88 & 7.95 & 52,389 & 34.50 & 1.1 & 763 & 0.12 & 0.004 & 11.1 \\
\hline-156.693 & 20.945 & $8: 51: 30$ & 24.90 & 7.95 & 52,351 & 34.47 & 1.0 & 763 & 0.11 & 0.004 & 11.1 \\
\hline-156.693 & 20.945 & 8:52:00 & 24.90 & 7.95 & 52,383 & 34.49 & 1.1 & 763 & 0.13 & 0.005 & 11.1 \\
\hline-156.693 & 20.945 & $8: 52: 30$ & 24.88 & 7.95 & 52,406 & 34.51 & 0.9 & 763 & 0.10 & 0.004 & 11.0 \\
\hline-156.693 & 20.945 & 8:53:00 & 24.88 & 7.94 & 52,389 & 34.50 & 0.9 & 763 & 0.09 & 0.004 & 11.0 \\
\hline-156.693 & 20.945 & $8: 53: 30$ & 24.89 & 7.96 & 52,401 & 34.51 & 0.9 & 763 & 0.08 & 0.004 & 11.0 \\
\hline-156.693 & 20.945 & 8:54:00 & 24.90 & 7.95 & 52,383 & 34.49 & 0.9 & 763 & 0.10 & 0.004 & 11.0 \\
\hline-156.693 & 20.944 & $8: 54: 30$ & 24.89 & 7.98 & 52,382 & 34.49 & 1.0 & 763 & 0.09 & 0.004 & 11.0 \\
\hline
\end{tabular}


Table A3. Nearshore wading survey with multiparameter water-quality sonde at Lahaina, Hawaii, May 6, 2008-Continued.

[Abbreviations: ${ }^{\circ} \mathrm{C}$, degrees Celsius; $\mu \mathrm{S} / \mathrm{cm}$, microSiemens per centimeter; NTU, nephelometric turbidity units; mg/L, milligrams per liter; mm Hg, millimeters mercury; $\mu \mathrm{g} / \mathrm{L}$, micrograms per liter; salinity is dimensionless; the entire dissolved oxygen record was deleted because the calibration was in error]

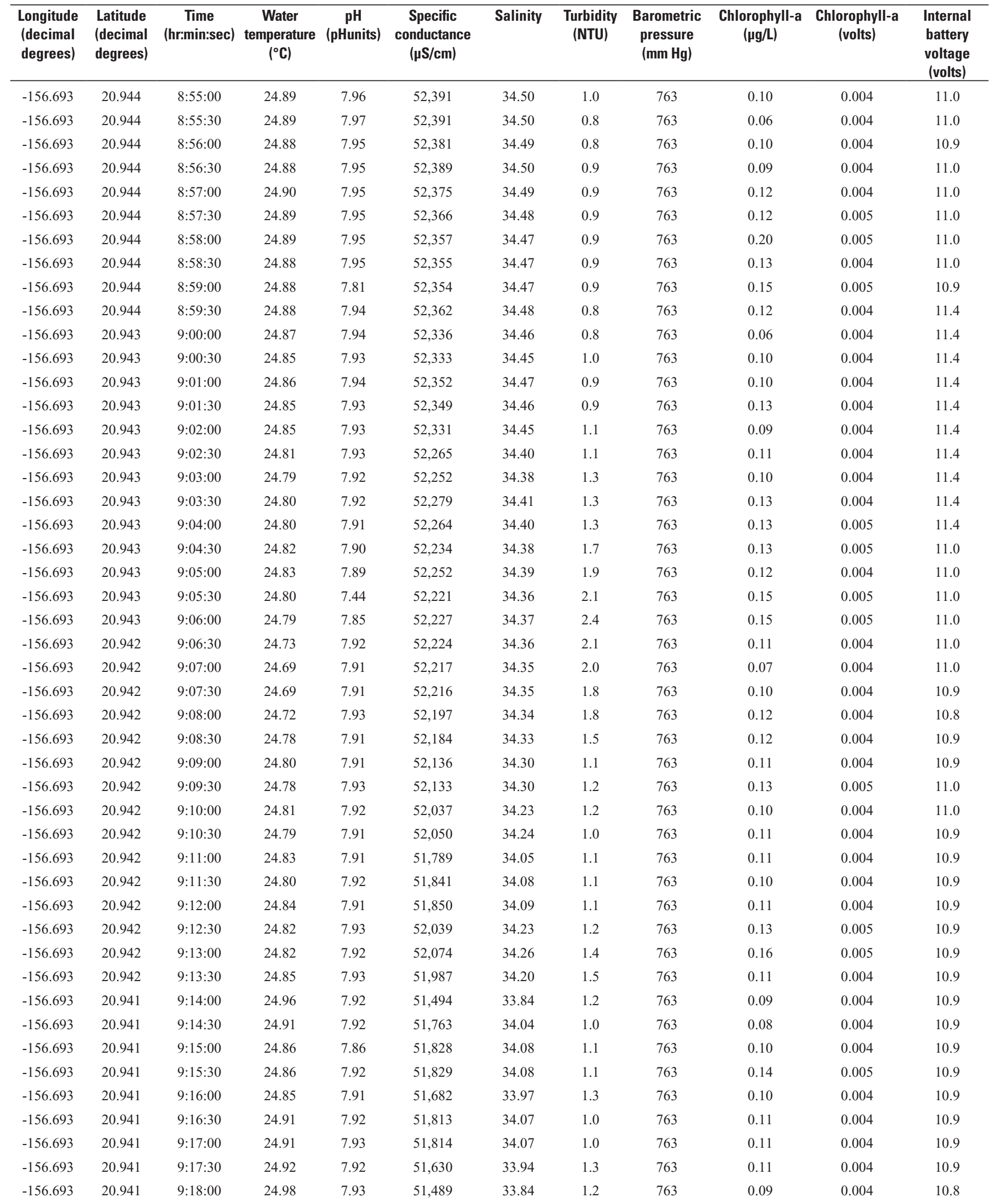


Table A3. Nearshore wading survey with multiparameter water-quality sonde at Lahaina, Hawaii, May 6, 2008 —Continued.

[Abbreviations: ${ }^{\circ} \mathrm{C}$, degrees Celsius; $\mu \mathrm{S} / \mathrm{cm}$, microSiemens per centimeter; NTU, nephelometric turbidity units; $\mathrm{mg} / \mathrm{L}$, milligrams per liter; $\mathrm{mm}$ Hg, millimeters mercury; $\mu \mathrm{g} / \mathrm{L}$, micrograms per liter; salinity is dimensionless; the entire dissolved oxygen record was deleted because the calibration was in error]

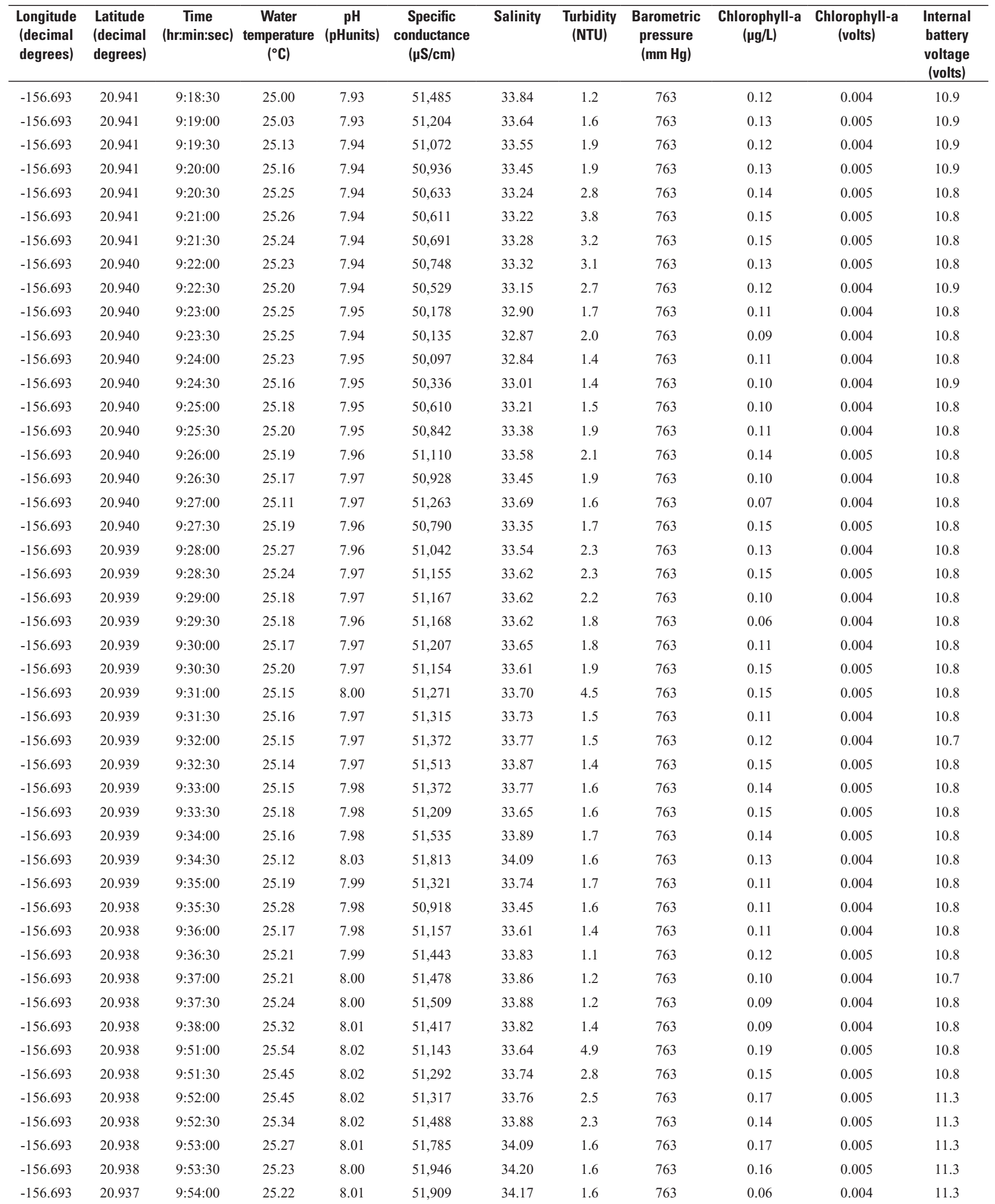


Table A3. Nearshore wading survey with multiparameter water-quality sonde at Lahaina, Hawaii, May 6, 2008 —Continued.

[Abbreviations: ${ }^{\circ} \mathrm{C}$, degrees Celsius; $\mu \mathrm{S} / \mathrm{cm}$, microSiemens per centimeter; NTU, nephelometric turbidity units; mg/L, milligrams per liter; mm Hg, millimeters mercury; $\mu \mathrm{g} / \mathrm{L}$, micrograms per liter; salinity is dimensionless; the entire dissolved oxygen record was deleted because the calibration was in error]

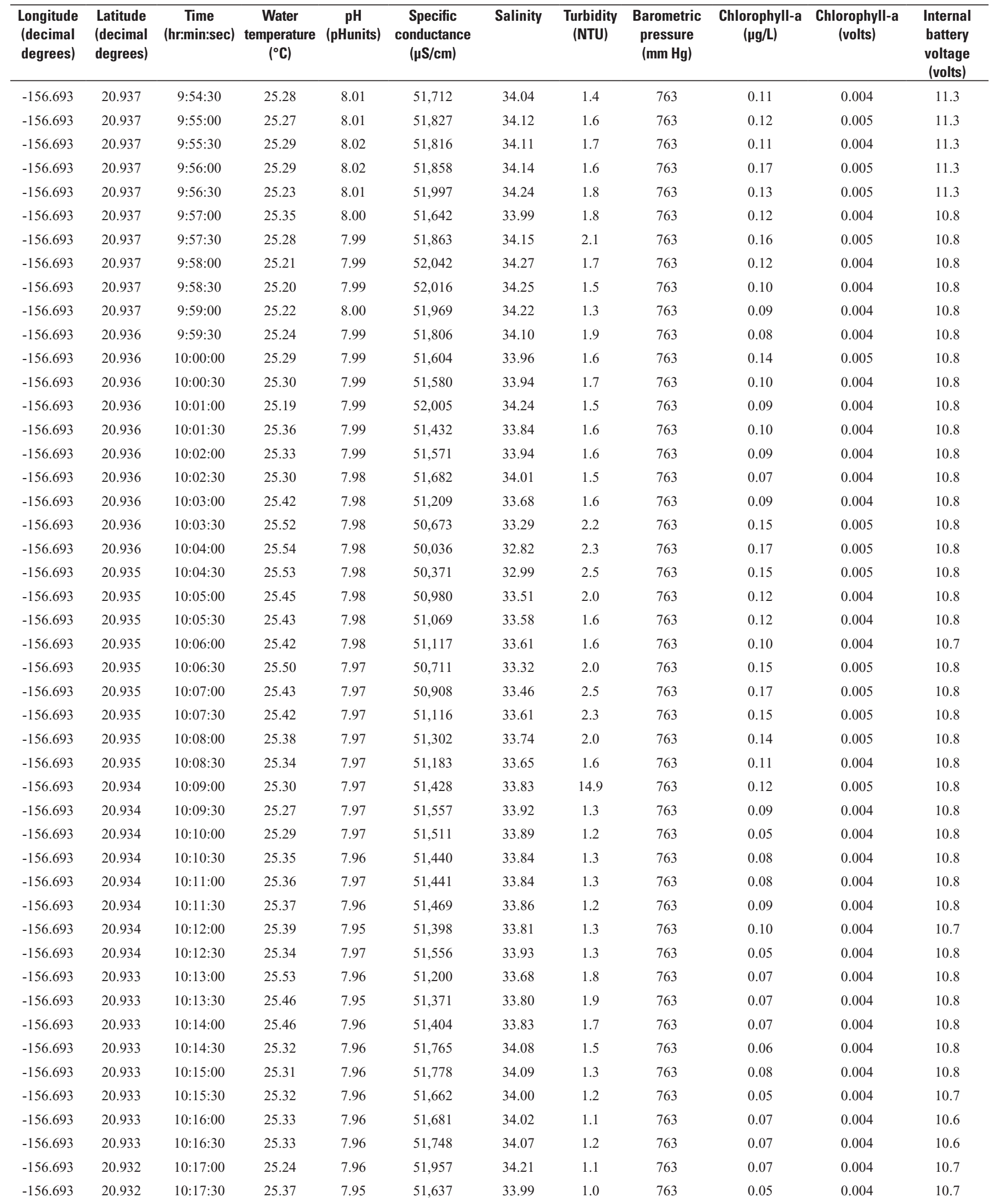


Table A3. Nearshore wading survey with multiparameter water-quality sonde at Lahaina, Hawaii, May 6, 2008 —Continued.

[Abbreviations: ${ }^{\circ} \mathrm{C}$, degrees Celsius; $\mu \mathrm{S} / \mathrm{cm}$, microSiemens per centimeter; NTU, nephelometric turbidity units; $\mathrm{mg} / \mathrm{L}$, milligrams per liter; $\mathrm{mm}$ Hg, millimeters mercury; $\mu \mathrm{g} / \mathrm{L}$, micrograms per liter; salinity is dimensionless; the entire dissolved oxygen record was deleted because the calibration was in error]

\begin{tabular}{|c|c|c|c|c|c|c|c|c|c|c|c|}
\hline $\begin{array}{l}\text { Longitude } \\
\text { (decimal } \\
\text { degrees) }\end{array}$ & $\begin{array}{l}\text { Latitude } \\
\text { (decimal } \\
\text { degrees) }\end{array}$ & $\begin{array}{c}\text { Time } \\
\text { (hr:min:sec) }\end{array}$ & $\begin{array}{c}\text { Water } \\
\text { temperature } \\
\left({ }^{\circ} \mathrm{C}\right)\end{array}$ & $\begin{array}{c}\mathrm{pH} \\
\text { (pHunits) }\end{array}$ & $\begin{array}{c}\text { Specific } \\
\text { conductance } \\
\text { ( } \mathrm{SS} / \mathrm{cm})\end{array}$ & Salinity & $\begin{array}{l}\text { Turbidity } \\
\text { (NTU) }\end{array}$ & $\begin{array}{c}\text { Barometric } \\
\text { pressure } \\
(\mathrm{mm} \mathrm{Hg})\end{array}$ & $\begin{array}{c}\text { Chlorophyll-a } \\
\text { ( } \mu \mathrm{g} / \mathrm{L})\end{array}$ & $\begin{array}{c}\text { Chlorophyll-a } \\
\text { (volts) }\end{array}$ & $\begin{array}{c}\text { Internal } \\
\text { battery } \\
\text { voltage } \\
\text { (volts) } \\
\end{array}$ \\
\hline-156.693 & 20.932 & $10: 18: 30$ & 25.35 & 7.95 & 51,693 & 34.03 & 1.0 & 763 & 0.06 & 0.004 & 10.7 \\
\hline-156.693 & 20.932 & 10:19:00 & 25.25 & 7.96 & 51,933 & 34.20 & 1.0 & 763 & 0.06 & 0.004 & 10.8 \\
\hline-156.694 & 20.932 & 10:19:30 & 25.30 & 7.95 & 51,868 & 34.15 & 1.0 & 763 & 0.07 & 0.004 & 10.8 \\
\hline-156.694 & 20.932 & $10: 20: 30$ & 25.35 & 7.95 & 51,693 & 34.03 & 1.1 & 763 & 0.07 & 0.004 & 10.7 \\
\hline-156.694 & 20.931 & $10: 21: 00$ & 25.30 & 7.95 & 51,851 & 34.14 & 1.2 & 763 & 0.08 & 0.004 & 10.7 \\
\hline-156.694 & 20.931 & $10: 21: 30$ & 25.29 & 7.96 & 51,934 & 34.20 & 1.0 & 763 & 0.07 & 0.004 & 10.7 \\
\hline-156.694 & 20.931 & $10: 22: 00$ & 25.26 & 7.96 & 51,995 & 34.24 & 0.9 & 763 & 0.06 & 0.004 & 10.7 \\
\hline-156.694 & 20.931 & $10: 22: 30$ & 25.26 & 7.96 & 52,021 & 34.26 & 0.8 & 763 & 0.06 & 0.004 & 10.4 \\
\hline-156.694 & 20.931 & $10: 24: 30$ & 25.23 & 7.95 & 52,098 & 34.32 & 0.9 & 763 & 0.07 & 0.004 & 10.7 \\
\hline-156.694 & 20.930 & $10: 25: 00$ & 25.17 & 7.96 & 52,171 & 34.36 & 0.8 & 763 & 0.07 & 0.004 & 10.7 \\
\hline-156.694 & 20.930 & $10: 25: 30$ & 25.14 & 7.96 & 52,232 & 34.41 & 0.7 & 763 & 0.07 & 0.004 & 10.7 \\
\hline-156.694 & 20.930 & $10: 26: 00$ & 25.13 & 7.96 & 52,220 & 34.40 & 0.7 & 763 & 0.07 & 0.004 & 10.5 \\
\hline-156.694 & 20.930 & $10: 26: 30$ & 25.13 & 7.96 & 52,230 & 34.40 & 0.6 & 763 & 0.06 & 0.004 & 10.7 \\
\hline-156.694 & 20.930 & $10: 27: 00$ & 25.21 & 7.96 & 52,094 & 34.31 & 0.6 & 763 & 0.07 & 0.004 & 10.7 \\
\hline-156.694 & 20.930 & $10: 27: 30$ & 25.23 & 7.96 & 52,081 & 34.30 & 0.7 & 763 & 0.07 & 0.004 & 10.7 \\
\hline-156.694 & 20.930 & $10: 28: 00$ & 25.22 & 7.96 & 52,155 & 34.36 & 0.7 & 763 & 0.07 & 0.004 & 10.7 \\
\hline-156.694 & 20.930 & $10: 28: 30$ & 25.40 & 7.95 & 52,134 & 34.36 & 1.0 & 763 & 0.07 & 0.004 & 10.7 \\
\hline-156.694 & 20.930 & 10:29:00 & 25.51 & 7.95 & 52,167 & 34.39 & 1.8 & 763 & 0.07 & 0.004 & 10.7 \\
\hline-156.695 & 20.929 & $10: 33: 00$ & 25.27 & 7.96 & 52,275 & 34.45 & 0.9 & 763 & 0.07 & 0.004 & 10.7 \\
\hline-156.695 & 20.929 & $10: 33: 30$ & 25.21 & 7.96 & 52,280 & 34.45 & 0.8 & 763 & 0.07 & 0.004 & 10.7 \\
\hline-156.695 & 20.929 & $10: 34: 00$ & 25.27 & 7.95 & 52,267 & 34.45 & 0.7 & 763 & 0.06 & 0.004 & 10.6 \\
\hline-156.695 & 20.929 & $10: 34: 30$ & 25.40 & 7.96 & 51,897 & 34.18 & 1.2 & 763 & 0.09 & 0.004 & 10.7 \\
\hline-156.696 & 20.929 & $10: 35: 00$ & 25.36 & 7.96 & 52,040 & 34.29 & 1.2 & 763 & 0.08 & 0.004 & 10.7 \\
\hline-156.696 & 20.929 & $10: 35: 30$ & 25.33 & 7.96 & 52,068 & 34.30 & 1.0 & 763 & 0.08 & 0.004 & 10.7 \\
\hline-156.696 & 20.929 & $10: 36: 00$ & 25.22 & 7.96 & 52,223 & 34.41 & 1.1 & 763 & 0.08 & 0.004 & 10.6 \\
\hline-156.696 & 20.929 & $10: 36: 30$ & 25.19 & 7.96 & 52,257 & 34.43 & 0.9 & 763 & 0.09 & 0.004 & 10.7 \\
\hline-156.696 & 20.929 & $10: 37: 00$ & 25.08 & 7.95 & 45,132 & 29.21 & 0.9 & 763 & 0.14 & 0.005 & 10.7 \\
\hline-156.696 & 20.929 & $10: 37: 30$ & 25.30 & 7.94 & 28,848 & 17.81 & 1.9 & 763 & 0.49 & 0.008 & 10.6 \\
\hline-156.696 & 20.929 & 10:38:00 & 25.30 & 7.96 & 20,741 & 12.41 & 3.1 & 763 & 0.59 & 0.009 & 10.6 \\
\hline-156.696 & 20.929 & $10: 38: 30$ & 25.26 & 7.92 & 35,589 & 22.44 & 3.4 & 763 & 0.54 & 0.008 & 10.6 \\
\hline-156.696 & 20.929 & 10:39:00 & 25.43 & 7.98 & 16,350 & 9.58 & 3.2 & 763 & 0.65 & 0.010 & 10.6 \\
\hline-156.696 & 20.929 & $10: 39: 30$ & 25.27 & 7.90 & 34,231 & 21.50 & 4.0 & 763 & 0.69 & 0.010 & 10.6 \\
\hline-156.696 & 20.929 & 10:40:00 & 25.47 & 7.96 & 14,949 & 8.70 & 3.9 & 763 & 0.87 & 0.012 & 10.6 \\
\hline-156.696 & 20.929 & $10: 40: 30$ & 25.42 & 7.95 & 18,292 & 10.83 & 4.5 & 763 & 0.88 & 0.012 & 10.6 \\
\hline-156.696 & 20.929 & $10: 41: 00$ & 25.37 & 7.90 & 25,857 & 15.80 & 4.6 & 763 & 0.94 & 0.012 & 11.1 \\
\hline
\end{tabular}


Table A3. Nearshore wading survey with multiparameter water-quality sonde at Lahaina, Hawaii, May 6, 2008—Continued.

[Abbreviations: ${ }^{\circ} \mathrm{C}$, degrees Celsius; $\mu \mathrm{S} / \mathrm{cm}$, microSiemens per centimeter; NTU, nephelometric turbidity units; mg/L, milligrams per liter; mm Hg, millimeters mercury; $\mu \mathrm{g} / \mathrm{L}$, micrograms per liter; salinity is dimensionless; the entire dissolved oxygen record was deleted because the calibration was in error]

\begin{tabular}{|c|c|c|c|c|c|c|c|c|c|c|c|}
\hline $\begin{array}{l}\text { Longitude } \\
\text { (decimal } \\
\text { degrees) }\end{array}$ & $\begin{array}{l}\text { Latitude } \\
\text { (decimal } \\
\text { degrees) }\end{array}$ & $\begin{array}{c}\text { Time } \\
\text { (hr:min:sec) }\end{array}$ & $\begin{array}{c}\text { Water } \\
\text { temperature } \\
\left({ }^{\circ} \mathrm{C}\right)\end{array}$ & $\begin{array}{c}\mathrm{pH} \\
\text { (pHunits) }\end{array}$ & $\begin{array}{c}\text { Specific } \\
\text { conductance } \\
(\mu \mathrm{S} / \mathrm{cm})\end{array}$ & Salinity & $\begin{array}{l}\text { Turbidity } \\
\text { (NTU) }\end{array}$ & $\begin{array}{c}\text { Barometric } \\
\text { pressure } \\
(\mathrm{mm} \mathrm{Hg})\end{array}$ & $\begin{array}{c}\text { Chlorophyll-a } \\
(\mu \mathrm{g} / \mathrm{L})\end{array}$ & $\begin{array}{l}\text { Chlorophyll-a } \\
\text { (volts) }\end{array}$ & $\begin{array}{c}\text { Internal } \\
\text { battery } \\
\text { voltage } \\
\text { (volts) } \\
\end{array}$ \\
\hline-156.696 & 20.929 & $10: 42: 00$ & 25.08 & 7.97 & 35,025 & 22.04 & 121.6 & 763 & 0.51 & 0.002 & 11.2 \\
\hline-156.696 & 20.929 & $10: 42: 30$ & 25.30 & 7.95 & 22,851 & 13.80 & 3.3 & 763 & 0.62 & 0.009 & 11.2 \\
\hline-156.696 & 20.929 & 10:43:00 & 25.22 & 7.91 & 40,800 & 26.73 & 4.3 & 763 & 0.49 & 0.008 & 11.3 \\
\hline-156.696 & 20.929 & $10: 44: 00$ & 25.21 & 7.92 & 42,310 & 27.19 & 2.9 & 763 & 0.54 & 0.008 & 11.3 \\
\hline-156.696 & 20.929 & $10: 48: 00$ & 25.17 & 7.97 & 33,390 & 20.91 & 5.2 & 763 & 0.32 & 0.007 & 11.2 \\
\hline-156.696 & 20.929 & $10: 48: 30$ & 25.22 & 7.92 & 39,889 & 25.47 & 2.5 & 763 & 0.55 & 0.009 & 11.3 \\
\hline-156.695 & 20.929 & 10:49:00 & 25.17 & 7.96 & 52,288 & 34.45 & 1.4 & 763 & 0.08 & 0.004 & 11.3 \\
\hline-156.695 & 20.929 & $10: 49: 30$ & 25.14 & 7.96 & 52,308 & 34.46 & 1.4 & 763 & 0.07 & 0.004 & 11.3 \\
\hline-156.695 & 20.929 & $10: 51: 30$ & 25.30 & 7.96 & 51,758 & 34.07 & 1.3 & 763 & 0.11 & 0.004 & 10.7 \\
\hline-156.695 & 20.929 & $10: 52: 00$ & 25.47 & 7.97 & 51,736 & 34.07 & 1.2 & 763 & 0.11 & 0.004 & 10.7 \\
\hline-156.695 & 20.929 & $10: 52: 30$ & 25.62 & 7.96 & 52,031 & 34.31 & 1.3 & 763 & 0.07 & 0.004 & 10.7 \\
\hline-156.695 & 20.929 & 10:53:00 & 25.59 & 7.96 & 52,032 & 34.30 & 1.6 & 763 & 0.08 & 0.004 & 10.7 \\
\hline-156.695 & 20.929 & $10: 53: 30$ & 25.67 & 7.96 & 51,983 & 34.27 & 1.7 & 763 & 0.09 & 0.004 & 10.7 \\
\hline-156.695 & 20.929 & $10: 54: 00$ & 25.59 & 7.96 & 52,050 & 34.32 & 1.7 & 763 & 0.08 & 0.004 & 11.1 \\
\hline-156.695 & 20.929 & $10: 54: 30$ & 25.59 & 7.96 & 52,134 & 34.38 & 1.4 & 763 & 0.06 & 0.004 & 11.2 \\
\hline-156.695 & 20.929 & 10:55:00 & 25.27 & 7.96 & 52,292 & 34.46 & 0.8 & 763 & 0.09 & 0.004 & 11.2 \\
\hline-156.694 & 20.929 & $10: 55: 30$ & 25.28 & 7.96 & 52,294 & 34.47 & 0.8 & 763 & 0.09 & 0.004 & 11.3 \\
\hline-156.694 & 20.929 & 10:56:00 & 25.41 & 7.96 & 52,289 & 34.48 & 0.9 & 763 & 0.06 & 0.004 & 11.3 \\
\hline-156.694 & 20.930 & 11:00:00 & 25.59 & 7.96 & 52,067 & 34.33 & 1.2 & 763 & 0.07 & 0.004 & 10.8 \\
\hline-156.694 & 20.930 & $11: 00: 30$ & 25.61 & 7.96 & 52,047 & 34.32 & 2.1 & 763 & 0.06 & 0.004 & 10.8 \\
\hline-156.694 & 20.931 & 11:01:00 & 25.46 & 7.95 & 52,080 & 34.33 & 3.4 & 763 & 0.08 & 0.004 & 10.7 \\
\hline-156.694 & 20.931 & 11:01:30 & 25.29 & 7.96 & 52,137 & 34.35 & 4.1 & 763 & 0.04 & 0.004 & 10.7 \\
\hline-156.694 & 20.931 & 11:02:00 & 25.33 & 7.96 & 52,035 & 34.28 & 3.3 & 763 & 0.05 & 0.004 & 10.7 \\
\hline-156.694 & 20.931 & $11: 02: 30$ & 25.42 & 7.96 & 51,946 & 34.22 & 3.9 & 763 & 0.06 & 0.004 & 10.7 \\
\hline-156.694 & 20.931 & 11:03:00 & 25.37 & 7.95 & 52,001 & 34.26 & 4.2 & 763 & 0.05 & 0.004 & 10.7 \\
\hline-156.694 & 20.931 & 11:03:30 & 25.27 & 7.96 & 52,166 & 34.37 & 6.4 & 763 & 0.05 & 0.004 & 10.5 \\
\hline-156.694 & 20.931 & 11:04:00 & 25.34 & 7.96 & 52,122 & 34.34 & 5.6 & 763 & 0.05 & 0.004 & 10.7 \\
\hline-156.694 & 20.931 & 11:04:30 & 25.34 & 7.96 & 52,172 & 34.38 & 7.0 & 763 & 0.06 & 0.004 & 10.7 \\
\hline-156.694 & 20.931 & 11:05:00 & 25.22 & 7.96 & 52,298 & 34.46 & 5.4 & 763 & 0.07 & 0.004 & 10.7 \\
\hline-156.693 & 20.932 & $11: 05: 30$ & 25.35 & 7.96 & 52,167 & 34.38 & 5.3 & 763 & 0.06 & 0.004 & 10.7 \\
\hline-156.693 & 20.932 & 11:06:00 & 25.39 & 7.96 & 52,156 & 34.37 & 4.4 & 763 & 0.07 & 0.004 & 10.6 \\
\hline-156.693 & 20.932 & $11: 06: 30$ & 25.30 & 7.97 & 52,189 & 34.39 & 3.9 & 763 & 0.06 & 0.004 & 10.7 \\
\hline-156.693 & 20.932 & 11:07:00 & 25.45 & 7.96 & 52,085 & 34.33 & 4.5 & 763 & 0.05 & 0.004 & 10.7 \\
\hline-156.693 & 20.932 & 11:07:30 & 25.37 & 7.96 & 52,111 & 34.34 & 4.2 & 763 & 0.06 & 0.004 & 10.7 \\
\hline-156.693 & 20.932 & 11:08:00 & 25.46 & 7.96 & 52,003 & 34.27 & 3.8 & 763 & 0.05 & 0.004 & 10.7 \\
\hline
\end{tabular}


Table A3. Nearshore wading survey with multiparameter water-quality sonde at Lahaina, Hawaii, May 6, 2008 —Continued.

[Abbreviations: ${ }^{\circ} \mathrm{C}$, degrees Celsius; $\mu \mathrm{S} / \mathrm{cm}$, microSiemens per centimeter; NTU, nephelometric turbidity units; $\mathrm{mg} / \mathrm{L}$, milligrams per liter; $\mathrm{mm}$ Hg, millimeters mercury; $\mu \mathrm{g} / \mathrm{L}$, micrograms per liter; salinity is dimensionless; the entire dissolved oxygen record was deleted because the calibration was in error]

\begin{tabular}{|c|c|c|c|c|c|c|c|c|c|c|c|}
\hline $\begin{array}{l}\text { Longitude } \\
\text { (decimal } \\
\text { degrees) }\end{array}$ & $\begin{array}{l}\text { Latitude } \\
\text { (decimal } \\
\text { degrees) }\end{array}$ & $\begin{array}{c}\text { Time } \\
\text { (hr:min:sec) }\end{array}$ & $\begin{array}{c}\text { Water } \\
\text { temperature } \\
\left({ }^{\circ} \mathrm{C}\right)\end{array}$ & $\begin{array}{c}\text { pH } \\
\text { (pHunits) }\end{array}$ & $\begin{array}{c}\text { Specific } \\
\text { conductance } \\
(\mu \mathrm{S} / \mathrm{cm})\end{array}$ & Salinity & $\begin{array}{c}\text { Turbidity } \\
\text { (NTU) }\end{array}$ & $\begin{array}{c}\text { Barometric } \\
\text { pressure } \\
(\mathrm{mm} \mathrm{Hg})\end{array}$ & $\begin{array}{c}\text { Chlorophyll-a } \\
(\mu \mathrm{g} / \mathrm{L})\end{array}$ & $\begin{array}{c}\text { Chlorophyll-a } \\
\text { (volts) }\end{array}$ & $\begin{array}{c}\text { Internal } \\
\text { battery } \\
\text { voltage } \\
\text { (volts) }\end{array}$ \\
\hline-156.693 & 20.932 & 11:09:00 & 25.48 & 7.96 & 51,976 & 34.25 & 12.2 & 763 & 0.05 & 0.004 & 10.6 \\
\hline-156.693 & 20.932 & 11:09:30 & 25.45 & 7.96 & 51,884 & 34.18 & 10.5 & 763 & 0.06 & 0.004 & 10.6 \\
\hline-156.693 & 20.933 & 11:10:00 & 25.44 & 7.96 & 51,906 & 34.19 & 9.5 & 763 & 0.06 & 0.004 & 10.6 \\
\hline-156.693 & 20.933 & 11:11:00 & 25.53 & 7.95 & 51,749 & 34.09 & 8.7 & 763 & 0.05 & 0.004 & 10.6 \\
\hline-156.693 & 20.933 & $11: 11: 30$ & 25.54 & 7.96 & 51,852 & 34.16 & 8.1 & 763 & 0.05 & 0.004 & 10.6 \\
\hline-156.693 & 20.933 & $11: 12: 00$ & 25.41 & 7.96 & 52,036 & 34.29 & 7.6 & 763 & 0.04 & 0.004 & 10.6 \\
\hline-156.693 & 20.933 & $11: 12: 30$ & 25.41 & 7.96 & 52,035 & 34.29 & 7.7 & 763 & 0.05 & 0.004 & 10.6 \\
\hline-156.693 & 20.933 & 11:13:00 & 25.41 & 7.96 & 52,068 & 34.31 & 7.5 & 763 & 0.04 & 0.004 & 10.6 \\
\hline-156.693 & 20.934 & 11:15:00 & 25.45 & 7.96 & 51,917 & 34.20 & 5.3 & 763 & 0.06 & 0.004 & 10.6 \\
\hline-156.693 & 20.934 & $11: 15: 30$ & 25.42 & 7.96 & 51,945 & 34.22 & 5.2 & 763 & 0.05 & 0.004 & 10.6 \\
\hline-156.693 & 20.934 & $11: 16: 00$ & 25.54 & 7.96 & 51,759 & 34.10 & 5.1 & 763 & 0.06 & 0.004 & 10.6 \\
\hline-156.693 & 20.934 & $11: 16: 30$ & 25.50 & 7.96 & 51,895 & 34.19 & 5.8 & 763 & 0.06 & 0.004 & 10.6 \\
\hline-156.693 & 20.934 & 11:17:00 & 25.49 & 7.96 & 51,960 & 34.24 & 6.1 & 763 & 0.06 & 0.004 & 10.6 \\
\hline-156.693 & 20.934 & $11: 17: 30$ & 25.47 & 7.97 & 51,930 & 34.22 & 5.7 & 763 & 0.06 & 0.004 & 10.6 \\
\hline-156.693 & 20.934 & 11:18:00 & 25.50 & 7.96 & 51,852 & 34.16 & 5.1 & 763 & 0.06 & 0.004 & 10.6 \\
\hline-156.693 & 20.935 & $11: 18: 30$ & 25.55 & 7.96 & 51,779 & 34.11 & 5.0 & 763 & 0.05 & 0.004 & 10.6 \\
\hline-156.693 & 20.935 & 11:19:00 & 25.62 & 7.96 & 51,709 & 34.07 & 4.9 & 763 & 0.07 & 0.004 & 10.6 \\
\hline-156.693 & 20.935 & $11: 19: 30$ & 25.60 & 7.97 & 51,662 & 34.03 & 4.5 & 763 & 0.05 & 0.004 & 10.6 \\
\hline-156.693 & 20.936 & $11: 23: 30$ & 25.65 & 7.97 & 51,529 & 33.94 & 4.6 & 763 & 0.08 & 0.004 & 10.6 \\
\hline-156.693 & 20.936 & $11: 24: 00$ & 25.48 & 7.98 & 51,823 & 34.14 & 4.7 & 763 & 0.08 & 0.004 & 10.6 \\
\hline-156.693 & 20.936 & $11: 24: 30$ & 25.64 & 7.98 & 51,334 & 33.79 & 5.0 & 763 & 0.09 & 0.004 & 10.6 \\
\hline-156.693 & 20.936 & $11: 25: 00$ & 25.72 & 7.97 & 51,157 & 33.67 & 5.0 & 763 & 0.10 & 0.004 & 10.6 \\
\hline-156.693 & 20.936 & $11: 25: 30$ & 25.75 & 7.98 & 51,010 & 33.56 & 5.4 & 763 & 0.11 & 0.004 & 10.6 \\
\hline-156.693 & 20.936 & $11: 26: 00$ & 25.75 & 7.98 & 50,985 & 33.54 & 5.5 & 763 & 0.13 & 0.005 & 10.6 \\
\hline-156.693 & 20.936 & $11: 26: 30$ & 25.73 & 7.98 & 51,108 & 33.63 & 5.3 & 763 & 0.14 & 0.005 & 10.5 \\
\hline-156.693 & 20.936 & $11: 27: 00$ & 25.78 & 7.98 & 50,958 & 33.53 & 5.4 & 763 & 0.13 & 0.005 & 10.6 \\
\hline-156.693 & 20.936 & $11: 27: 30$ & 25.64 & 7.98 & 51,333 & 33.79 & 5.4 & 763 & 0.11 & 0.004 & 10.4 \\
\hline-156.693 & 20.936 & $11: 28: 00$ & 25.48 & 8.00 & 51,729 & 34.03 & 4.6 & 763 & 0.07 & 0.004 & 10.6 \\
\hline-156.693 & 20.936 & $11: 28: 30$ & 25.51 & 7.99 & 51,635 & 34.00 & 4.4 & 763 & 0.08 & 0.004 & 10.6 \\
\hline-156.693 & 20.936 & $11: 29: 00$ & 25.59 & 7.99 & 51,297 & 33.76 & 4.7 & 763 & 0.08 & 0.004 & 10.6 \\
\hline-156.693 & 20.937 & $11: 29: 30$ & 25.53 & 7.99 & 51,521 & 33.92 & 5.4 & 763 & 0.09 & 0.004 & 10.6 \\
\hline
\end{tabular}


Table A4. Nearshore kayak survey with multiparameter water-quality sonde at Lahaina, Hawaii, May 23, 2008.

[Abbreviations: ${ }^{\circ} \mathrm{C}$, degrees Celsius; $\mathrm{mg} / \mathrm{L}$, milligrams per liter; $\mathrm{mm} \mathrm{Hg}$, millimeters mercury; $\mu \mathrm{g} / \mathrm{L}$, micrograms per liter; deleted data include chlorophyll values displaying an out-of-range flag and the entire records of specific conductance, salinity, and turbidity, for which highly erratic readings indicated the sensors did not remain fully submerged]

\begin{tabular}{|c|c|c|c|c|c|c|c|c|c|c|}
\hline $\begin{array}{l}\text { Longitude } \\
\text { (decimal } \\
\text { degrees) }\end{array}$ & $\begin{array}{l}\text { Latitude } \\
\text { (decimal } \\
\text { degrees) }\end{array}$ & $\begin{array}{c}\text { Time } \\
\text { (hr:min:sec) }\end{array}$ & $\begin{array}{c}\text { Water } \\
\text { temperature } \\
\left({ }^{\circ} \mathrm{C}\right)\end{array}$ & $\begin{array}{c}\mathrm{pH} \\
\text { (pH units) }\end{array}$ & $\begin{array}{l}\text { Dissolved } \\
\text { oxygen } \\
\text { saturation } \\
\text { (percent) }\end{array}$ & $\begin{array}{c}\text { Dissolved } \\
\text { oxygen } \\
\text { (mg/L) }\end{array}$ & $\begin{array}{c}\text { Barometric } \\
\text { pressure } \\
(\mathrm{mm} \mathrm{Hg})\end{array}$ & $\begin{array}{l}\text { Chlorophyll-a } \\
(\mu \mathrm{g} / \mathrm{L})\end{array}$ & $\begin{array}{l}\text { Chlorophyll-a } \\
\text { (volts) }\end{array}$ & $\begin{array}{c}\text { Internal } \\
\text { battery } \\
\text { voltage } \\
\text { (volts) }\end{array}$ \\
\hline-156.691 & 20.949 & 7:36:00 & 25.17 & 7.80 & 91.3 & 6.22 & 763 & 0.41 & 0.007 & 10.1 \\
\hline-156.691 & 20.949 & $7: 36: 30$ & 25.18 & 7.81 & 90.6 & 6.17 & 763 & 0.43 & 0.007 & 10.1 \\
\hline-156.691 & 20.949 & 7:37:00 & 25.17 & 7.82 & 90.4 & 6.16 & 763 & 0.43 & 0.007 & 10.1 \\
\hline-156.692 & 20.949 & $7: 38: 30$ & 25.10 & 7.81 & 91.1 & 7.46 & 763 & 0.85 & 0.011 & 10.1 \\
\hline-156.692 & 20.948 & 7:39:00 & 25.16 & 7.82 & 85.4 & 7.04 & 763 & 0.34 & 0.005 & 10.1 \\
\hline-156.692 & 20.948 & $7: 39: 30$ & 25.14 & 7.81 & 85.1 & 6.21 & 763 & 0.17 & 0.007 & 10.1 \\
\hline-156.692 & 20.948 & 7:40:00 & 25.04 & 7.82 & 88.1 & 6.01 & 763 & 0.53 & 0.008 & 10.1 \\
\hline-156.692 & 20.947 & $7: 40: 30$ & 24.84 & 7.84 & 94.3 & 6.45 & 763 & 0.63 & 0.009 & 10.2 \\
\hline-156.693 & 20.945 & 7:43:00 & 25.16 & 7.88 & 93.8 & 6.84 & 763 & 0.42 & 0.007 & 10.1 \\
\hline-156.693 & 20.945 & $7: 43: 30$ & 25.22 & 7.90 & 96.0 & 6.56 & 763 & 0.27 & 0.002 & 10.1 \\
\hline-156.693 & 20.945 & 7:44:00 & 25.27 & 7.90 & 96.7 & 7.01 & 763 & 0.13 & 0.009 & 10.1 \\
\hline-156.693 & 20.944 & $7: 44: 30$ & 25.30 & 7.92 & 96.4 & 7.93 & 763 & 0.01 & 0.007 & 10.1 \\
\hline-156.693 & 20.944 & $7: 45: 00$ & 25.26 & 7.90 & 94.3 & 7.49 & 763 & 0.37 & 0.002 & 10.1 \\
\hline-156.693 & 20.944 & $7: 45: 30$ & 25.26 & 7.90 & 94.9 & 6.65 & 763 & & 0.004 & 10.1 \\
\hline-156.693 & 20.943 & 7:46:00 & 25.24 & 7.89 & 93.2 & 6.62 & 763 & & 0.003 & 10.1 \\
\hline-156.693 & 20.943 & $7: 46: 30$ & 25.28 & 7.89 & 91.2 & 6.19 & 763 & & 0.010 & 10.1 \\
\hline-156.693 & 20.943 & 7:47:00 & 25.33 & 7.89 & 91.0 & 7.48 & 763 & 0.18 & 0.005 & 10.1 \\
\hline-156.693 & 20.942 & $7: 47: 30$ & 25.27 & 7.89 & 92.1 & 7.59 & 763 & 0.37 & 0.005 & 10.1 \\
\hline-156.693 & 20.942 & 7:48:00 & 25.28 & 7.89 & 90.5 & 6.67 & 763 & 0.11 & 0.009 & 10.1 \\
\hline-156.693 & 20.939 & $7: 52: 30$ & 25.42 & 7.91 & 93.9 & 6.83 & 763 & 1.06 & 0.004 & 10.1 \\
\hline-156.693 & 20.938 & 7:53:00 & 25.40 & 7.92 & 95.4 & 7.78 & 763 & 0.60 & 0.009 & 10.1 \\
\hline-156.693 & 20.938 & $7: 53: 30$ & 25.42 & 7.93 & 97.1 & 6.96 & 763 & 0.43 & 0.007 & 10.1 \\
\hline-156.693 & 20.937 & 7:54:00 & 25.42 & 7.94 & 98.9 & 7.35 & 763 & 0.31 & 0.011 & 10.1 \\
\hline-156.693 & 20.937 & $7: 54: 30$ & 25.46 & 7.94 & 98.7 & 6.94 & 763 & 0.65 & 0.009 & 10.1 \\
\hline-156.693 & 20.937 & $7: 55: 00$ & 25.46 & 7.94 & 98.3 & 8.06 & 763 & 0.35 & 0.006 & 10.1 \\
\hline-156.693 & 20.936 & $7: 55: 30$ & 25.42 & 7.94 & 97.3 & 7.43 & 763 & 0.47 & 0.011 & 10.1 \\
\hline-156.693 & 20.936 & 7:56:00 & 25.41 & 7.94 & 98.2 & 7.14 & 763 & 0.25 & 0.006 & 10.1 \\
\hline-156.693 & 20.936 & $7: 56: 30$ & 25.41 & 7.94 & 97.1 & 7.29 & 763 & 0.30 & 0.009 & 10.1 \\
\hline-156.693 & 20.935 & 7:57:00 & 25.38 & 7.94 & 97.6 & 6.63 & 763 & 0.28 & 0.006 & 10.1 \\
\hline-156.693 & 20.935 & $7: 57: 30$ & 25.35 & 7.93 & 97.9 & 7.48 & 763 & 0.27 & 0.006 & 10.1 \\
\hline-156.693 & 20.934 & 7:58:00 & 25.36 & 7.94 & 98.0 & 7.08 & 763 & 0.31 & 0.006 & 10.1 \\
\hline-156.693 & 20.934 & $7: 58: 30$ & 25.35 & 7.94 & 98.4 & 7.79 & 763 & 0.17 & 0.005 & 10.1 \\
\hline-156.693 & 20.934 & 7:59:00 & 25.31 & 7.94 & 98.4 & 6.83 & 763 & 0.26 & 0.006 & 10.1 \\
\hline-156.693 & 20.933 & $7: 59: 30$ & 25.30 & 7.94 & 98.4 & 8.03 & 763 & & 0.003 & 10.1 \\
\hline-156.693 & 20.933 & 8:00:00 & 25.29 & 7.94 & 98.5 & 8.07 & 763 & 0.16 & 0.005 & 10.1 \\
\hline-156.693 & 20.933 & 8:00:30 & 25.30 & 7.94 & 98.4 & 6.71 & 763 & 0.19 & 0.004 & 10.1 \\
\hline-156.693 & 20.932 & 8:01:00 & 25.30 & 7.94 & 98.6 & 7.04 & 763 & 0.04 & 0.005 & 10.1 \\
\hline
\end{tabular}


Table A4. Nearshore kayak survey with multiparameter water-quality sonde at Lahaina, Hawaii, May 23, 2008 —Continued.

[Abbreviations: ${ }^{\circ} \mathrm{C}$, degrees Celsius; $\mathrm{mg} / \mathrm{L}$, milligrams per liter; $\mathrm{mm} \mathrm{Hg}$, millimeters mercury; $\mu \mathrm{g} / \mathrm{L}$, micrograms per liter; deleted data include chlorophyll values displaying an out-of-range flag and the entire records of specific conductance, salinity, and turbidity, for which highly erratic readings indicated the sensors did not remain fully submerged]

\begin{tabular}{|c|c|c|c|c|c|c|c|c|c|c|}
\hline $\begin{array}{l}\text { Longitude } \\
\text { (decimal } \\
\text { degrees) }\end{array}$ & $\begin{array}{l}\text { Latitude } \\
\text { (decimal } \\
\text { degrees) }\end{array}$ & $\begin{array}{c}\text { Time } \\
\text { (hr:min:sec) }\end{array}$ & $\begin{array}{c}\text { Water } \\
\text { temperature } \\
\left({ }^{\circ} \mathrm{C}\right)\end{array}$ & $\begin{array}{c}\mathrm{pH} \\
\text { (pH units) }\end{array}$ & $\begin{array}{l}\text { Dissolved } \\
\text { oxygen } \\
\text { saturation } \\
\text { (percent) }\end{array}$ & $\begin{array}{c}\text { Dissolved } \\
\text { oxygen } \\
\text { (mg/L) }\end{array}$ & $\begin{array}{c}\text { Barometric } \\
\text { pressure } \\
(\mathrm{mm} \mathrm{Hg})\end{array}$ & $\begin{array}{c}\text { Chlorophyll-a } \\
(\mu \mathrm{g} / \mathrm{L})\end{array}$ & $\begin{array}{c}\text { Chlorophyll-a } \\
\text { (volts) }\end{array}$ & $\begin{array}{c}\text { Internal } \\
\text { battery } \\
\text { voltage } \\
\text { (volts) }\end{array}$ \\
\hline-156.693 & 20.932 & 8:02:00 & 25.30 & 7.94 & 98.7 & 7.26 & 763 & 0.26 & 0.005 & 10.1 \\
\hline-156.694 & 20.931 & $8: 02: 30$ & 25.33 & 7.95 & 98.7 & 6.69 & 763 & 0.00 & 0.003 & 10.1 \\
\hline-156.694 & 20.931 & 8:03:00 & 25.33 & 7.94 & 99.0 & 6.73 & 763 & 0.19 & 0.005 & 10.1 \\
\hline-156.694 & 20.930 & 8:04:30 & 25.35 & 7.95 & 99.0 & 7.15 & 763 & 0.27 & 0.006 & 10.1 \\
\hline-156.694 & 20.930 & 8:05:00 & 25.37 & 7.94 & 99.5 & 6.75 & 763 & 0.56 & 0.007 & 10.1 \\
\hline-156.694 & 20.929 & $8: 05: 30$ & 25.35 & 7.95 & 98.6 & 6.73 & 763 & 0.32 & 0.006 & 10.1 \\
\hline-156.695 & 20.929 & 8:06:00 & 25.33 & 7.95 & 99.0 & 6.71 & 763 & 0.25 & 0.006 & 10.1 \\
\hline-156.695 & 20.929 & 8:06:30 & 25.33 & 7.95 & 99.1 & 6.72 & 763 & 0.37 & 0.007 & 10.1 \\
\hline-156.694 & 20.930 & 8:09:00 & 25.32 & 7.95 & 99.1 & 6.72 & 763 & 0.44 & 0.004 & 10.1 \\
\hline-156.694 & 20.931 & 8:09:30 & 25.32 & 7.95 & 98.9 & 6.71 & 763 & 0.48 & 0.007 & 10.1 \\
\hline-156.694 & 20.931 & $8: 10: 00$ & 25.30 & 7.95 & 99.0 & 7.15 & 763 & 0.37 & 0.012 & 10.1 \\
\hline-156.694 & 20.932 & $8: 10: 30$ & 25.30 & 7.95 & 99.1 & 6.72 & 763 & 0.23 & 0.002 & 10.1 \\
\hline-156.694 & 20.932 & $8: 11: 00$ & 25.30 & 7.95 & 98.8 & 6.71 & 763 & 0.31 & 0.002 & 10.1 \\
\hline-156.693 & 20.932 & $8: 11: 30$ & 25.32 & 7.94 & 98.9 & 6.71 & 763 & 0.10 & 0.004 & 10.1 \\
\hline-156.693 & 20.933 & $8: 12: 00$ & 25.30 & 7.95 & 98.9 & 8.14 & 763 & 0.36 & 0.006 & 10.1 \\
\hline-156.693 & 20.933 & $8: 12: 30$ & 25.32 & 7.95 & 99.1 & 7.26 & 763 & & 0.003 & 10.1 \\
\hline-156.693 & 20.934 & $8: 13: 00$ & 25.33 & 7.95 & 99.0 & 8.03 & 763 & & 0.005 & 10.1 \\
\hline-156.693 & 20.934 & $8: 13: 30$ & 25.34 & 7.95 & 98.7 & 6.84 & 763 & 0.36 & 0.006 & 10.1 \\
\hline-156.693 & 20.934 & $8: 14: 00$ & 25.35 & 7.94 & 98.8 & 8.12 & 763 & 0.43 & 0.007 & 10.1 \\
\hline-156.693 & 20.938 & $8: 18: 30$ & 25.39 & 7.95 & 100.2 & 6.80 & 763 & 0.27 & 0.006 & 10.1 \\
\hline-156.693 & 20.938 & $8: 19: 00$ & 25.40 & 7.95 & 100.6 & 6.82 & 763 & 0.38 & 0.007 & 10.1 \\
\hline-156.693 & 20.939 & $8: 19: 30$ & 25.43 & 7.95 & 100.5 & 6.82 & 763 & 0.44 & 0.008 & 10.1 \\
\hline-156.693 & 20.939 & 8:20:00 & 25.44 & 7.94 & 98.7 & 6.70 & 763 & 0.43 & 0.007 & 10.1 \\
\hline-156.693 & 20.939 & $8: 20: 30$ & 25.44 & 7.92 & 96.5 & 6.55 & 763 & 0.41 & 0.007 & 10.1 \\
\hline-156.693 & 20.940 & $8: 21: 00$ & 25.43 & 7.90 & 92.3 & 6.27 & 763 & 0.38 & 0.007 & 10.1 \\
\hline-156.693 & 20.940 & $8: 21: 30$ & 25.43 & 7.90 & 90.4 & 6.14 & 763 & 0.31 & 0.006 & 10.1 \\
\hline-156.693 & 20.940 & $8: 22: 00$ & 25.42 & 7.89 & 89.8 & 6.29 & 763 & 0.32 & 0.006 & 10.1 \\
\hline-156.693 & 20.941 & $8: 22: 30$ & 25.43 & 7.90 & 88.7 & 6.04 & 763 & 0.23 & 0.006 & 10.1 \\
\hline-156.693 & 20.941 & $8: 23: 00$ & 25.40 & 7.91 & 91.6 & 6.22 & 763 & 0.25 & 0.006 & 10.1 \\
\hline-156.693 & 20.941 & $8: 23: 30$ & 25.39 & 7.90 & 90.5 & 6.15 & 763 & 0.26 & 0.005 & 10.1 \\
\hline-156.693 & 20.942 & $8: 24: 00$ & 25.39 & 7.90 & 91.2 & 6.19 & 763 & 0.04 & 0.004 & 10.1 \\
\hline-156.693 & 20.942 & $8: 24: 30$ & 25.39 & 7.90 & 91.9 & 6.24 & 763 & 0.40 & 0.010 & 10.1 \\
\hline-156.693 & 20.942 & $8: 25: 00$ & 25.36 & 7.90 & 92.1 & 6.25 & 763 & 0.36 & 0.007 & 10.1 \\
\hline-156.693 & 20.943 & $8: 25: 30$ & 25.40 & 7.91 & 92.1 & 6.24 & 763 & 0.17 & 0.006 & 10.1 \\
\hline-156.693 & 20.943 & $8: 26: 00$ & 25.41 & 7.91 & 92.4 & 7.53 & 763 & 0.13 & 0.008 & 10.1 \\
\hline-156.693 & 20.943 & $8: 26: 30$ & 25.32 & 7.91 & 92.1 & 6.25 & 763 & 1.02 & 0.008 & 10.1 \\
\hline-156.693 & 20.944 & $8: 27: 00$ & 25.37 & 7.92 & 92.1 & 6.24 & 763 & 0.40 & 0.007 & 10.1 \\
\hline
\end{tabular}


Table A4. Nearshore kayak survey with multiparameter water-quality sonde at Lahaina, Hawaii, May 23, 2008_-Continued.

[Abbreviations: ${ }^{\circ} \mathrm{C}$, degrees Celsius; $\mathrm{mg} / \mathrm{L}$, milligrams per liter; $\mathrm{mm} \mathrm{Hg}$, millimeters mercury; $\mu \mathrm{g} / \mathrm{L}$, micrograms per liter; deleted data include chlorophyll values displaying an out-of-range flag and the entire records of specific conductance, salinity, and turbidity, for which highly erratic readings indicated the sensors did not remain fully submerged]

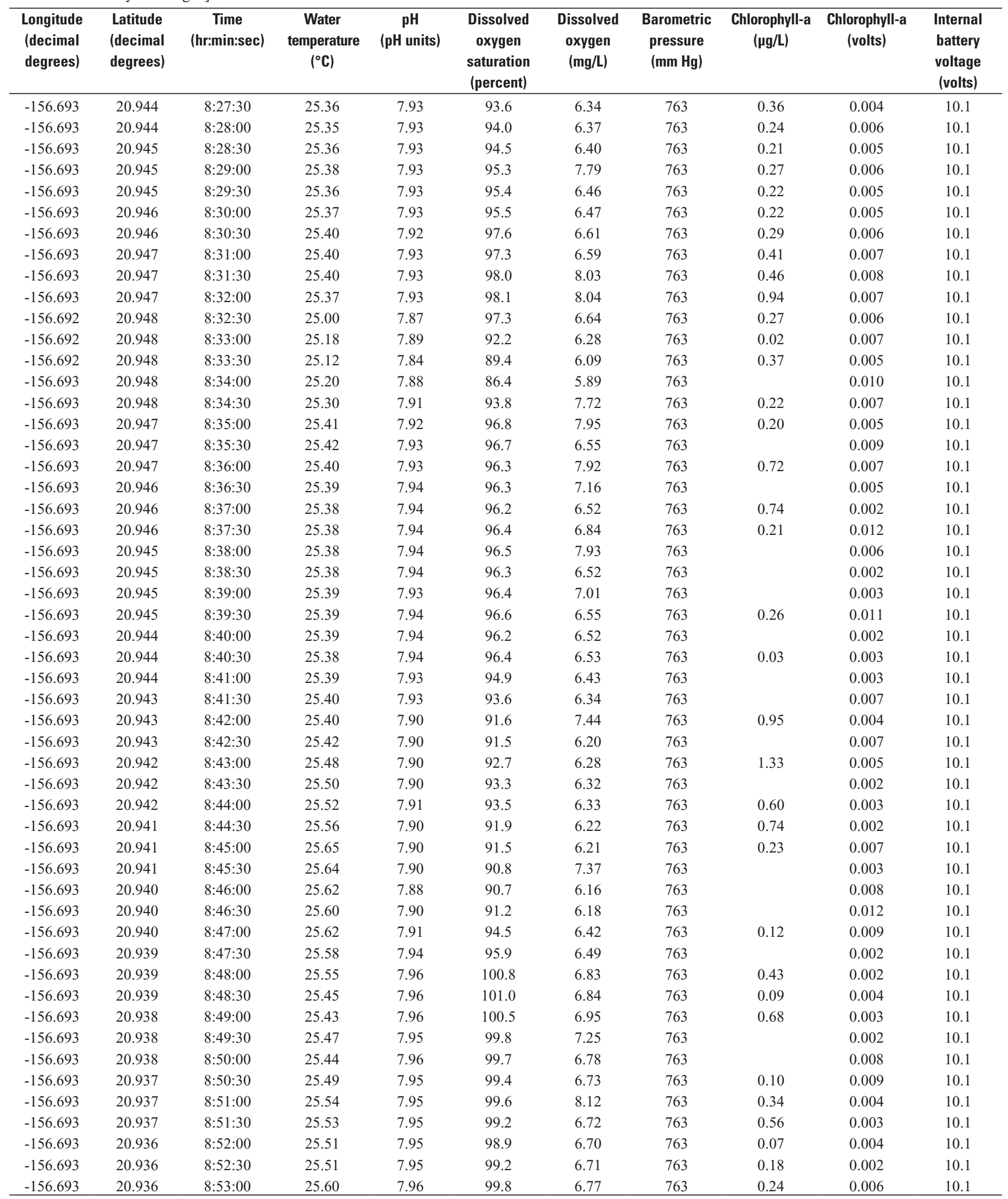




\section{Appendix B. \\ Maps of Laboratory Results and Field Measurements for Water and Algae Samples at Kihei, Hawaii}




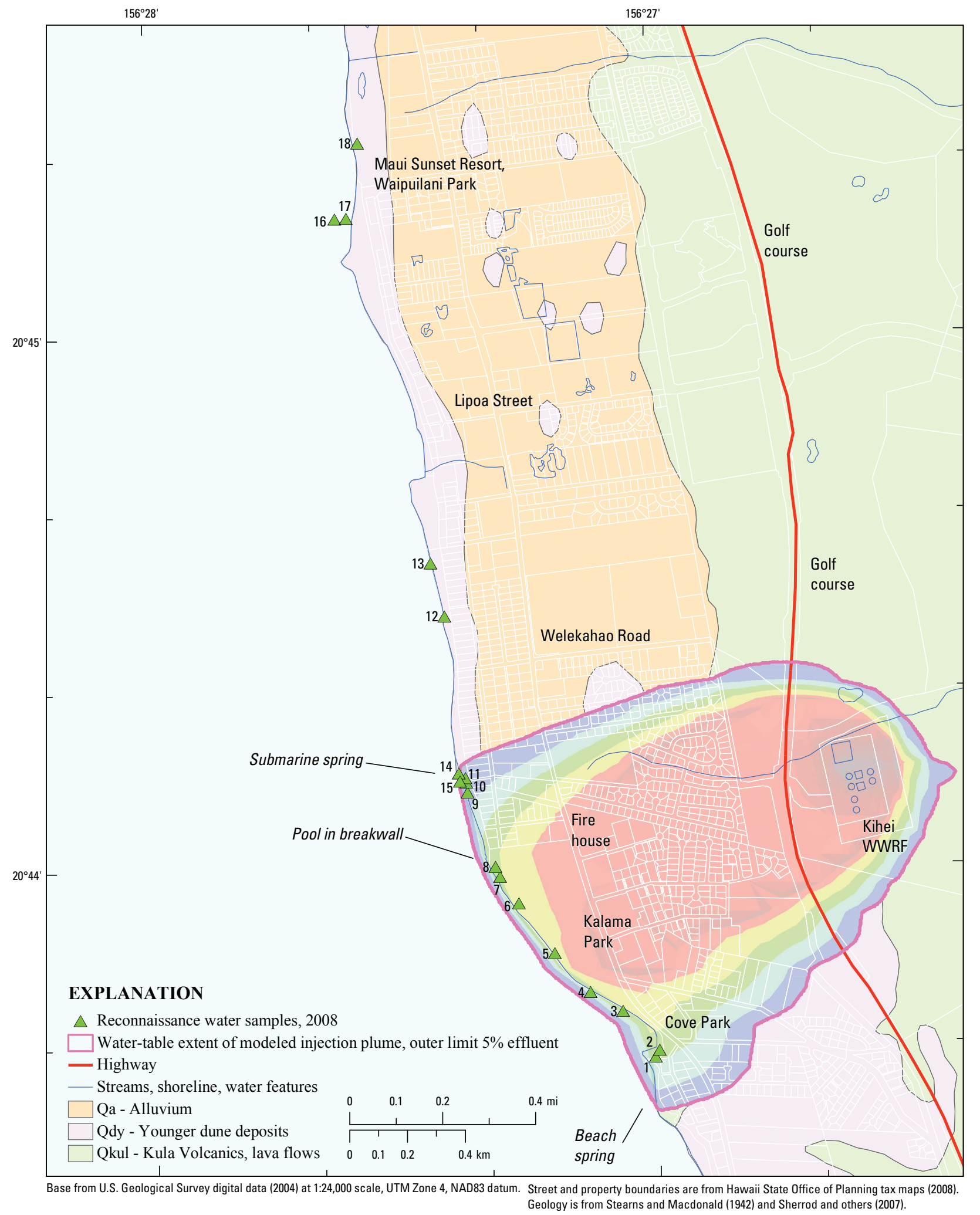

Figure B1. Reconnaissance water samples at Kihei, Hawaii. 


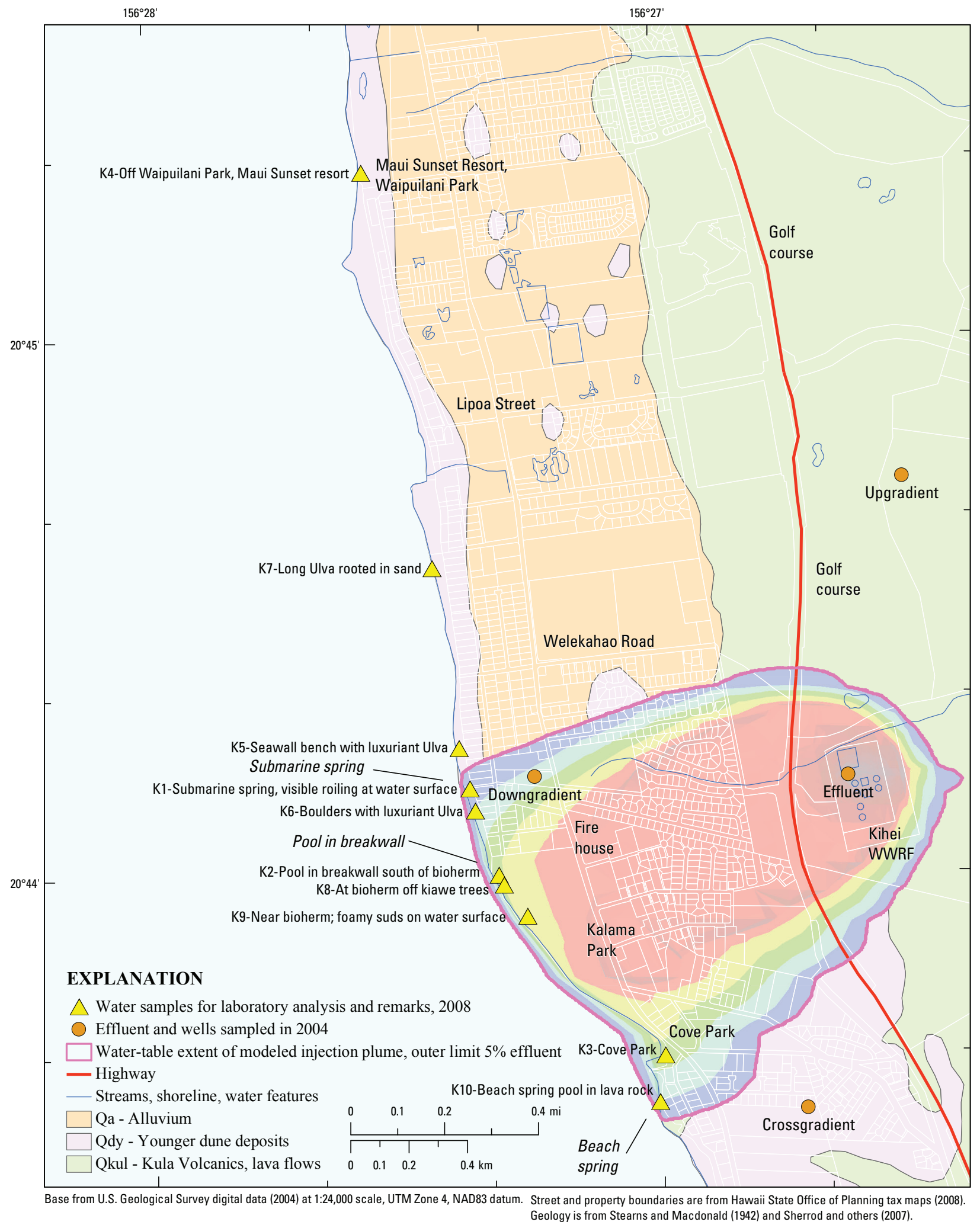

Figure B2. Laboratory water samples (with remarks on their field context) at Kihei, Hawaii. 


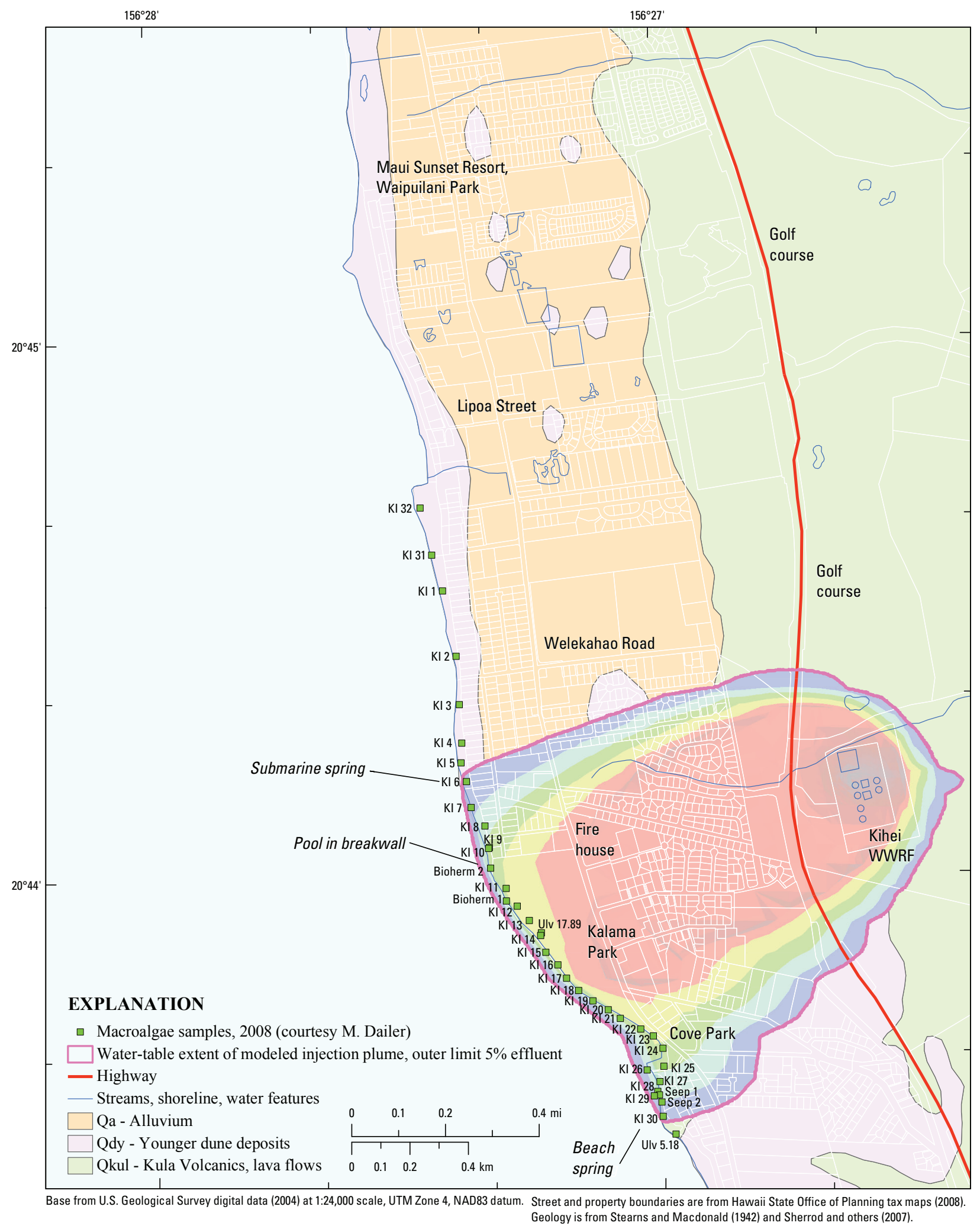

Figure B3. Macroalgae samples at Kihei, Hawaii. 


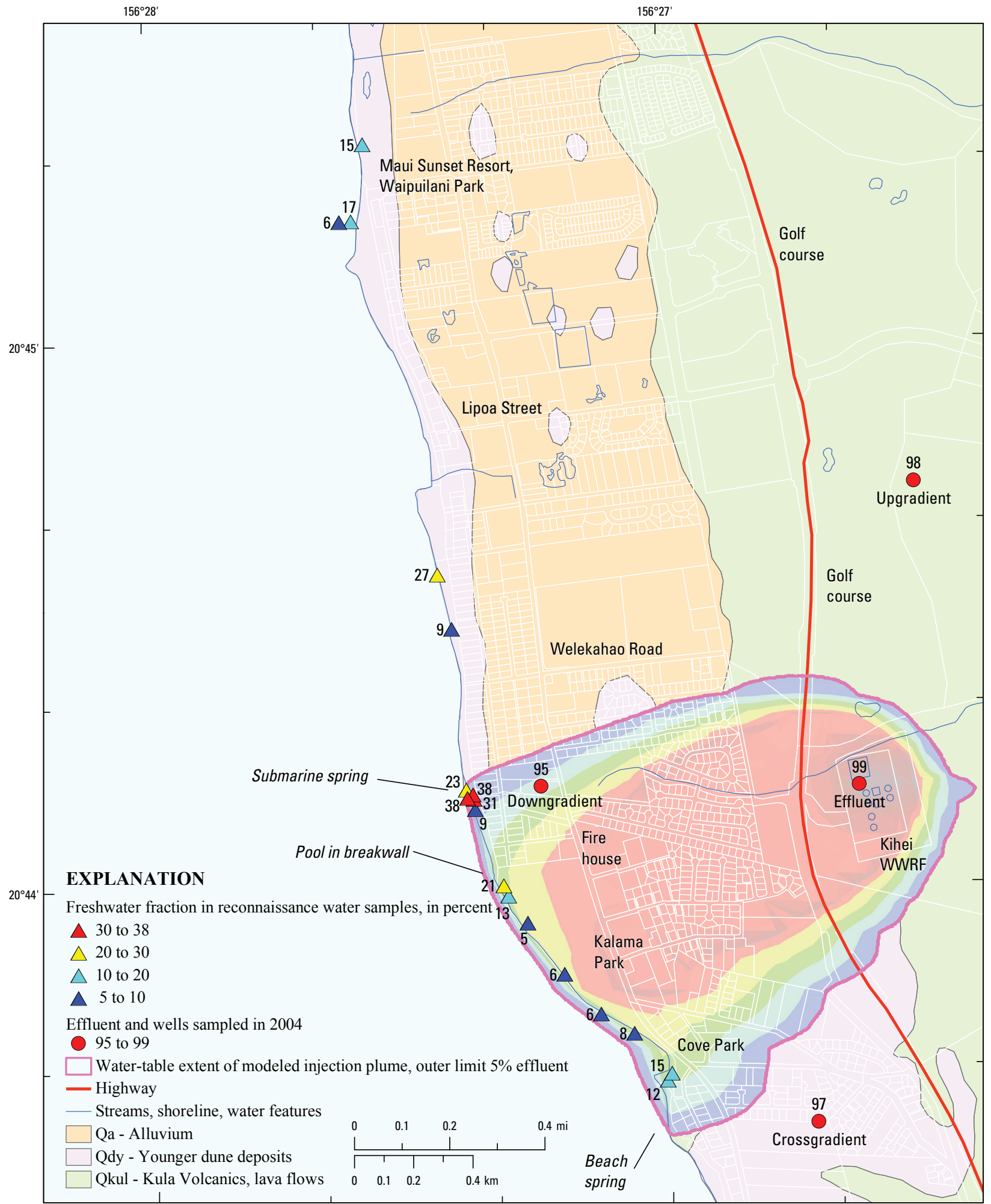

Base from U.S. Geological Survey digital data (2004) at 1:24,000 scale, UTM Zone 4, NAD83 datum. Street and property boundaries are from Hawaii State 0ffice of Planning tax maps (2008). Geology is from Stearns and Macdonald (1942) and Sherrod and others (2007).

Figure B4. Freshwater fraction (computed from salinity) in reconnaissance water samples at Kihei, Hawaii. 


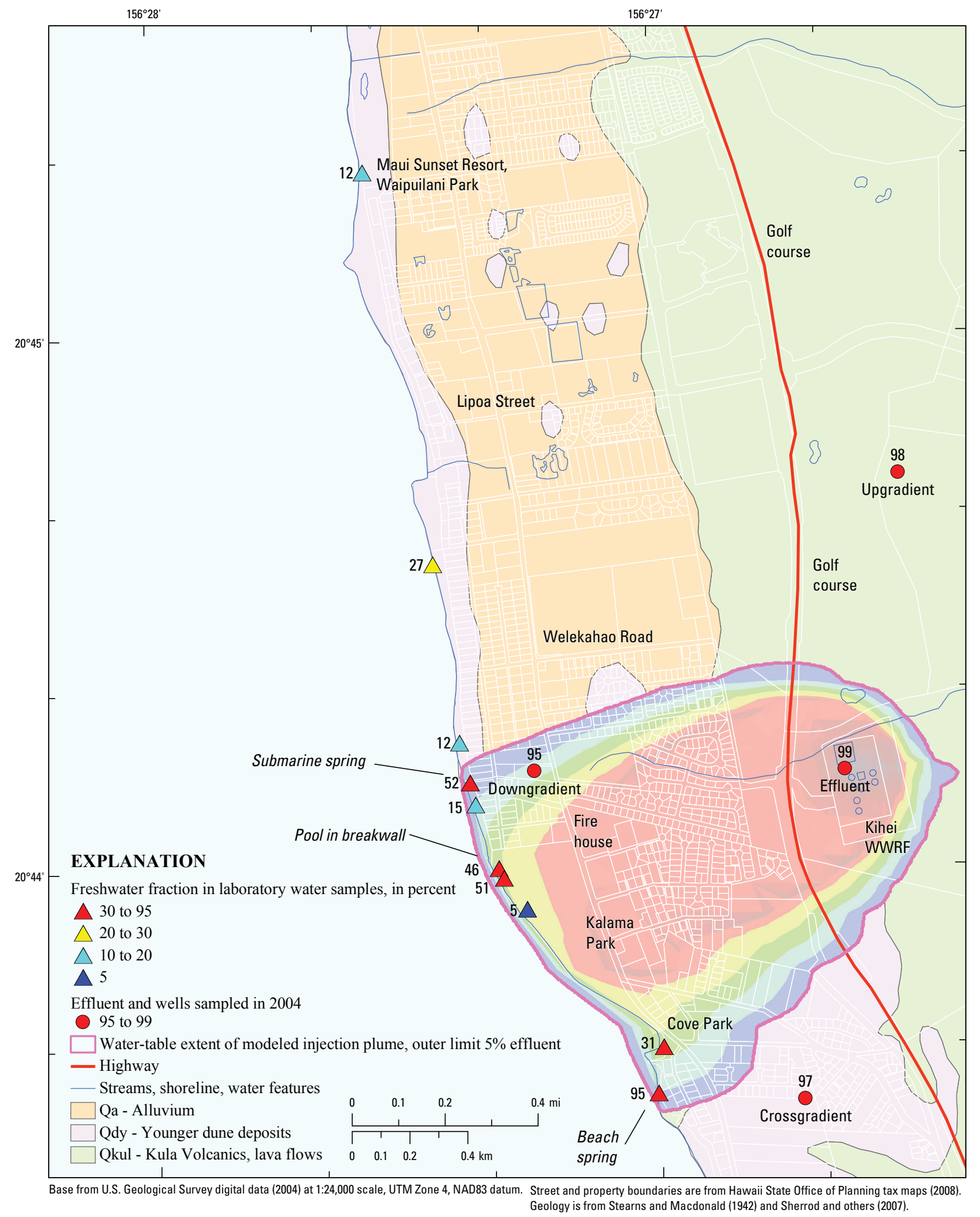

Figure B5. Freshwater fraction (computed from salinity) in laboratory water samples at Kihei, Hawaii. 


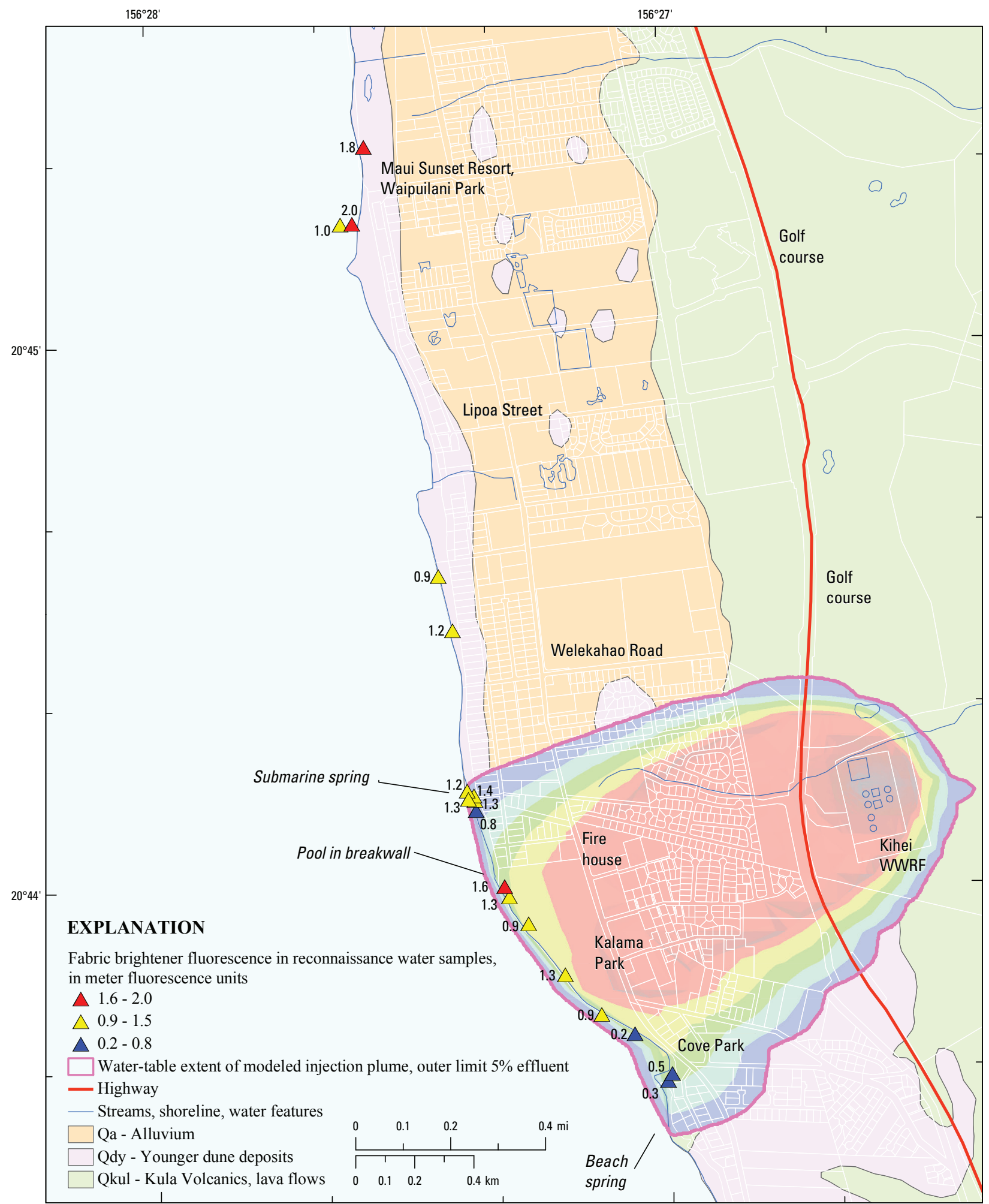

Base from U.S. Geological Survey digital data (2004) at 1:24,000 scale, UTM Zone 4, NAD83 datum. Street and property boundaries are from Hawaii State 0ffice of Planning tax maps (2008). Geology is from Stearns and Macdonald (1942) and Sherrod and others (2007).

Figure B6. Fabric brightener fluorescence in reconnaissance water samples at Kihei, Hawaii. 


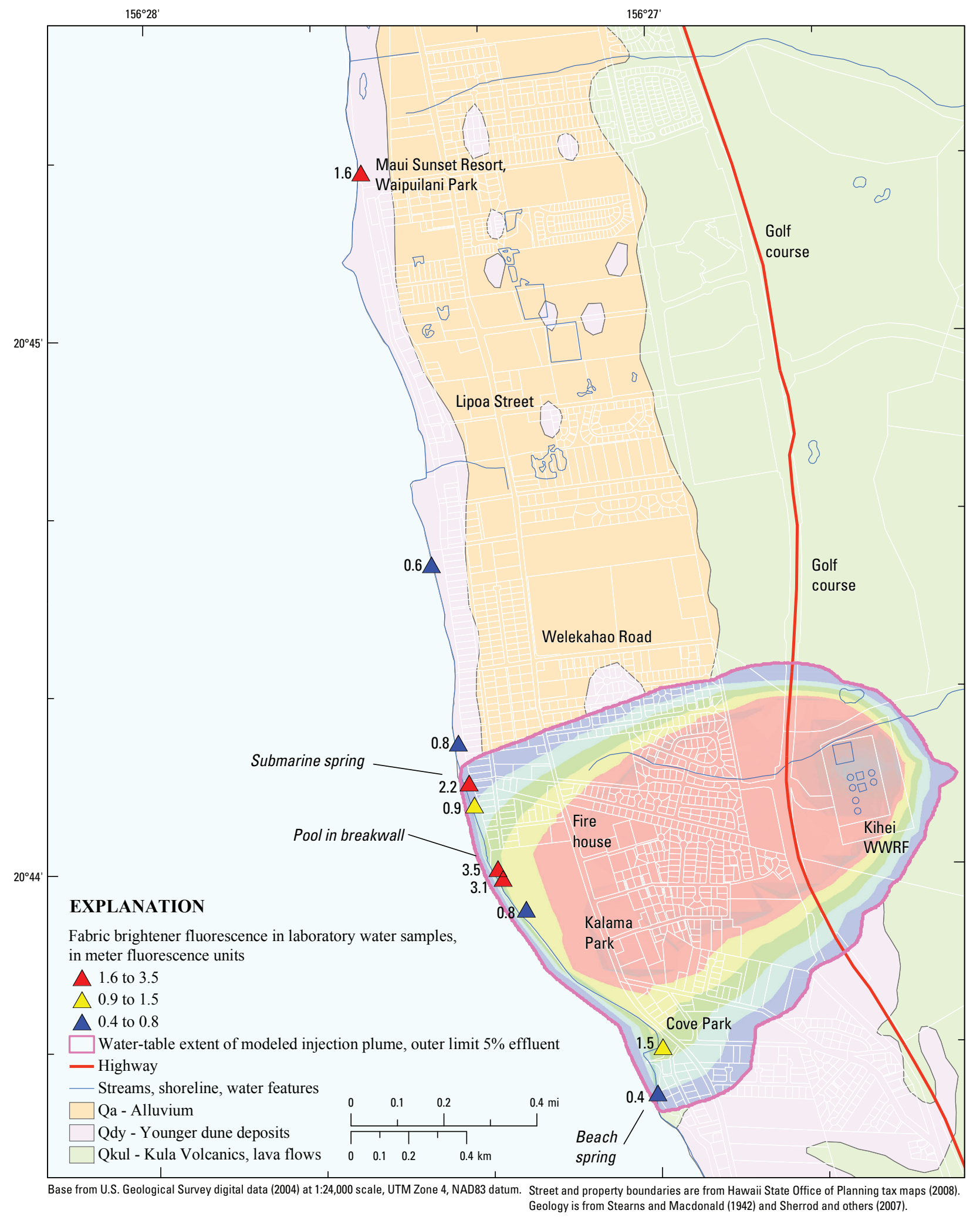

Figure B7. Fabric brightener fluorescence in laboratory water samples at Kihei, Hawaii. 


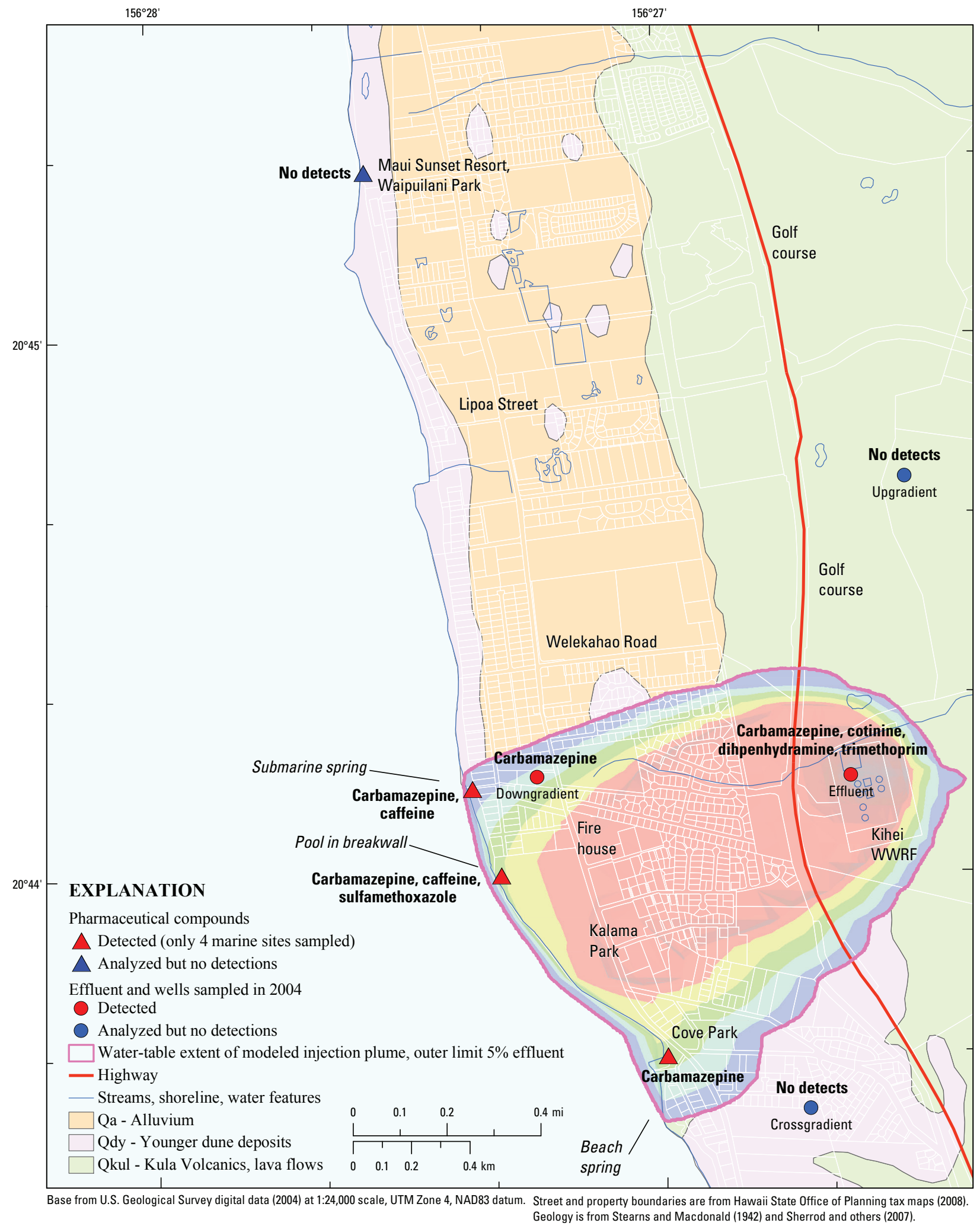

Figure B8. Pharmaceutical compounds in water at Kihei, Hawaii. 


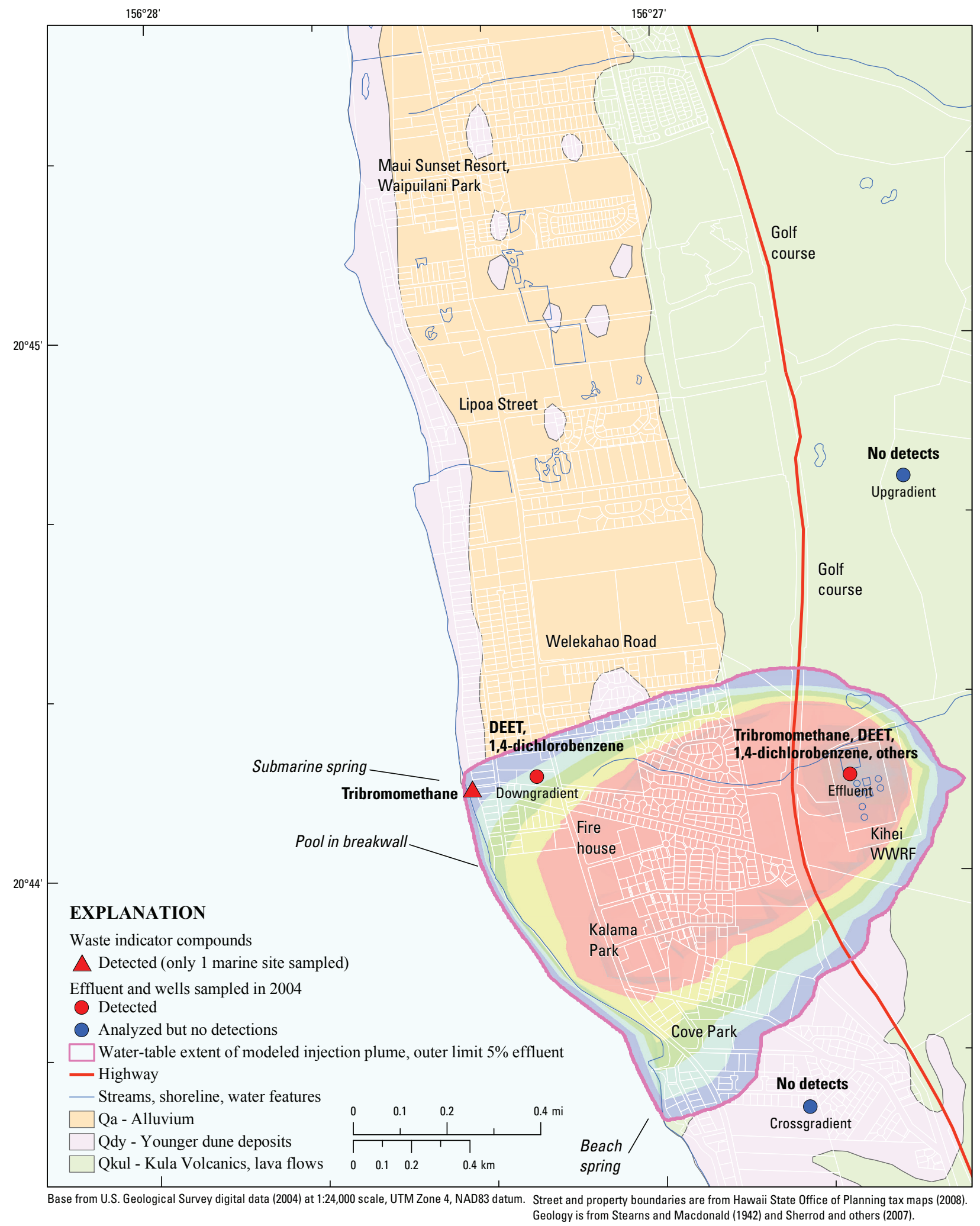

Figure B9. Waste indicator compounds in water at Kihei, Hawaii. 


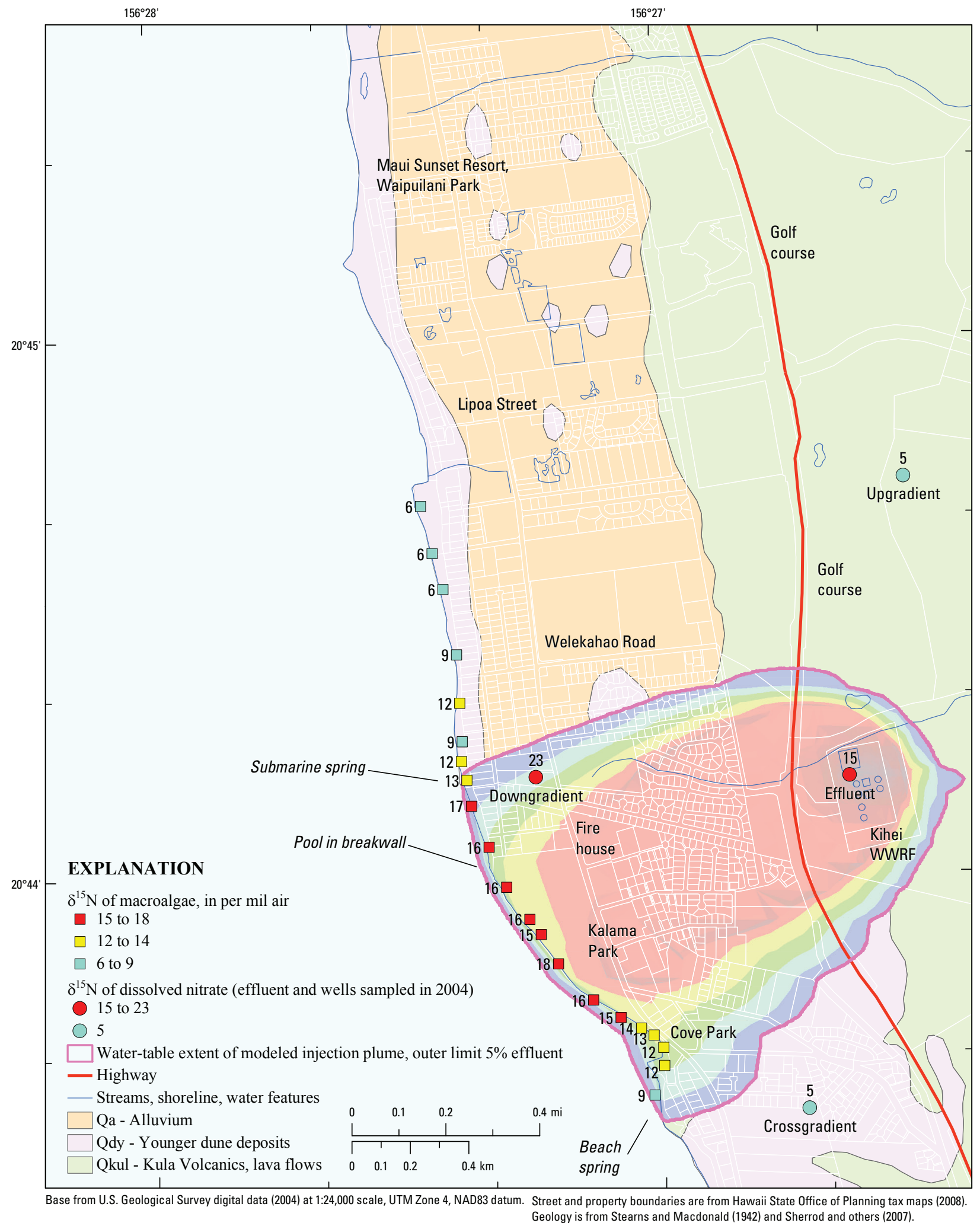

Figure B10. $\delta^{15} \mathrm{~N}$ isotopic composition of macroalgae at Kihei, Hawaii. 


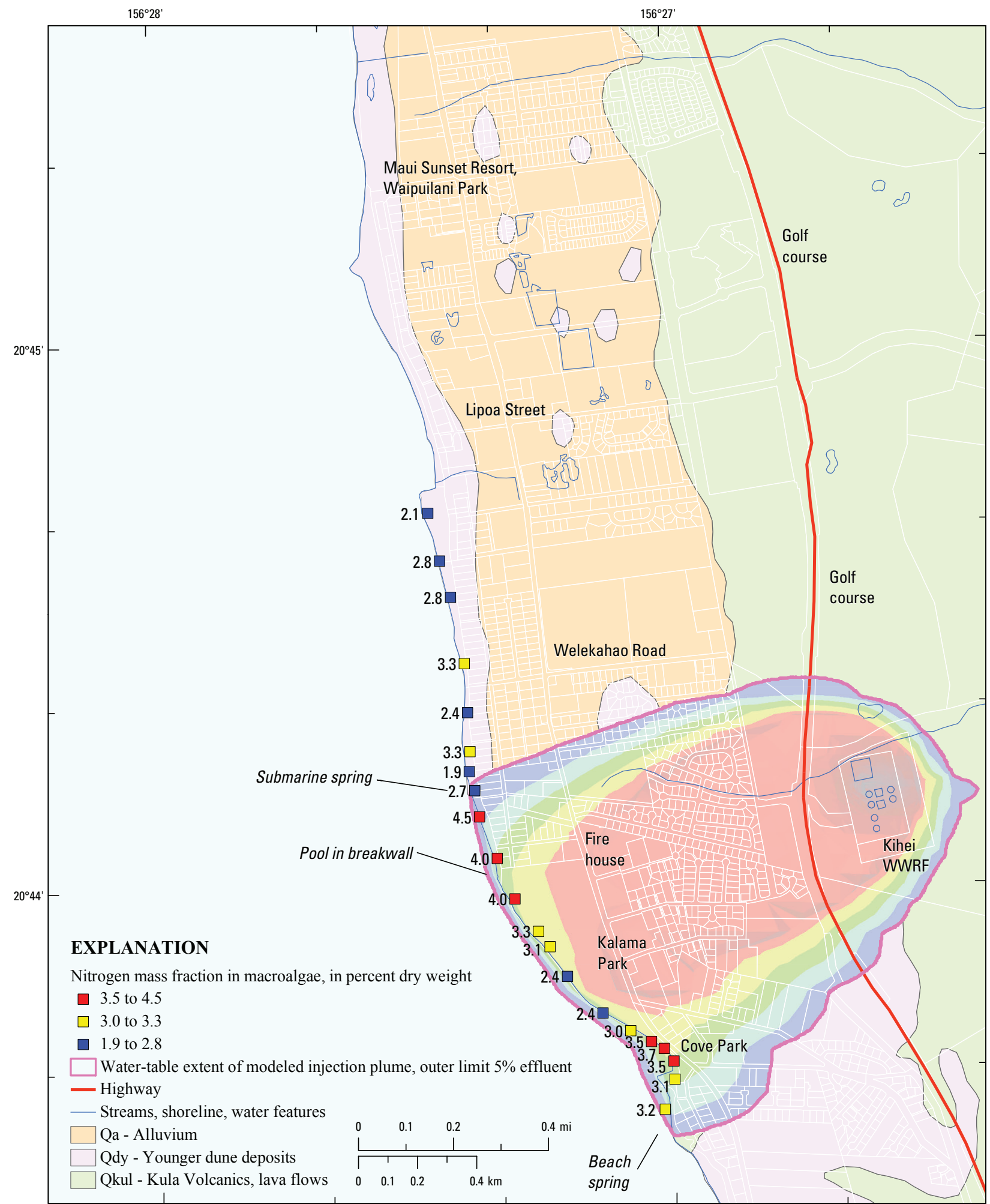

Base from U.S. Geological Survey digital data (2004) at 1:24,000 scale, UTM Zone 4, NAD83 datum. Street and property boundaries are from Hawaii State 0ffice of Planning tax maps (2008). Geology is from Stearns and Macdonald (1942) and Sherrod and others (2007).

Figure B11. Nitrogen mass fraction in macroalgae at Kihei, Hawaii. 


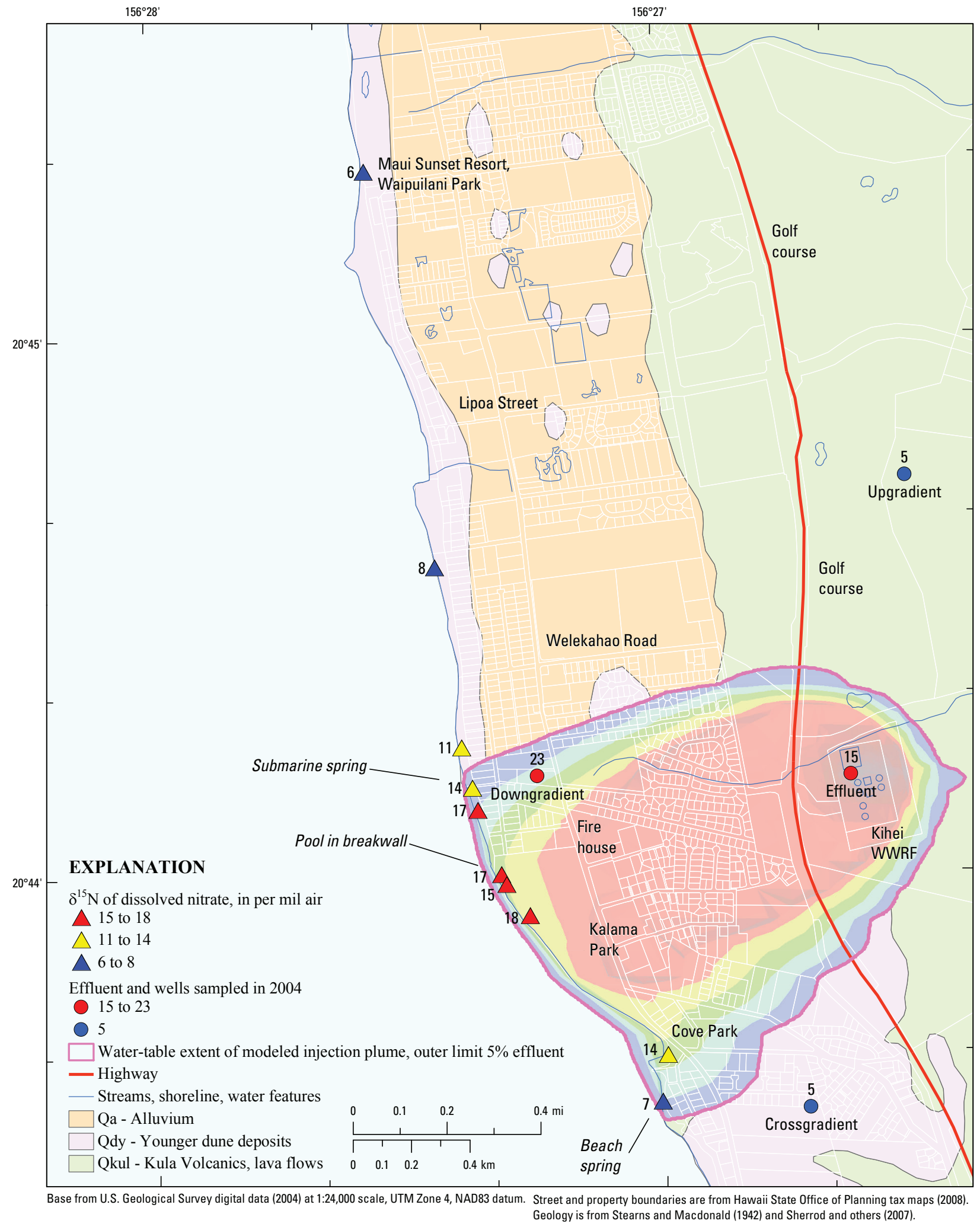

Figure B12. $\delta^{15} \mathrm{~N}$ isotopic composition of dissolved nitrate at Kihei, Hawaii. 


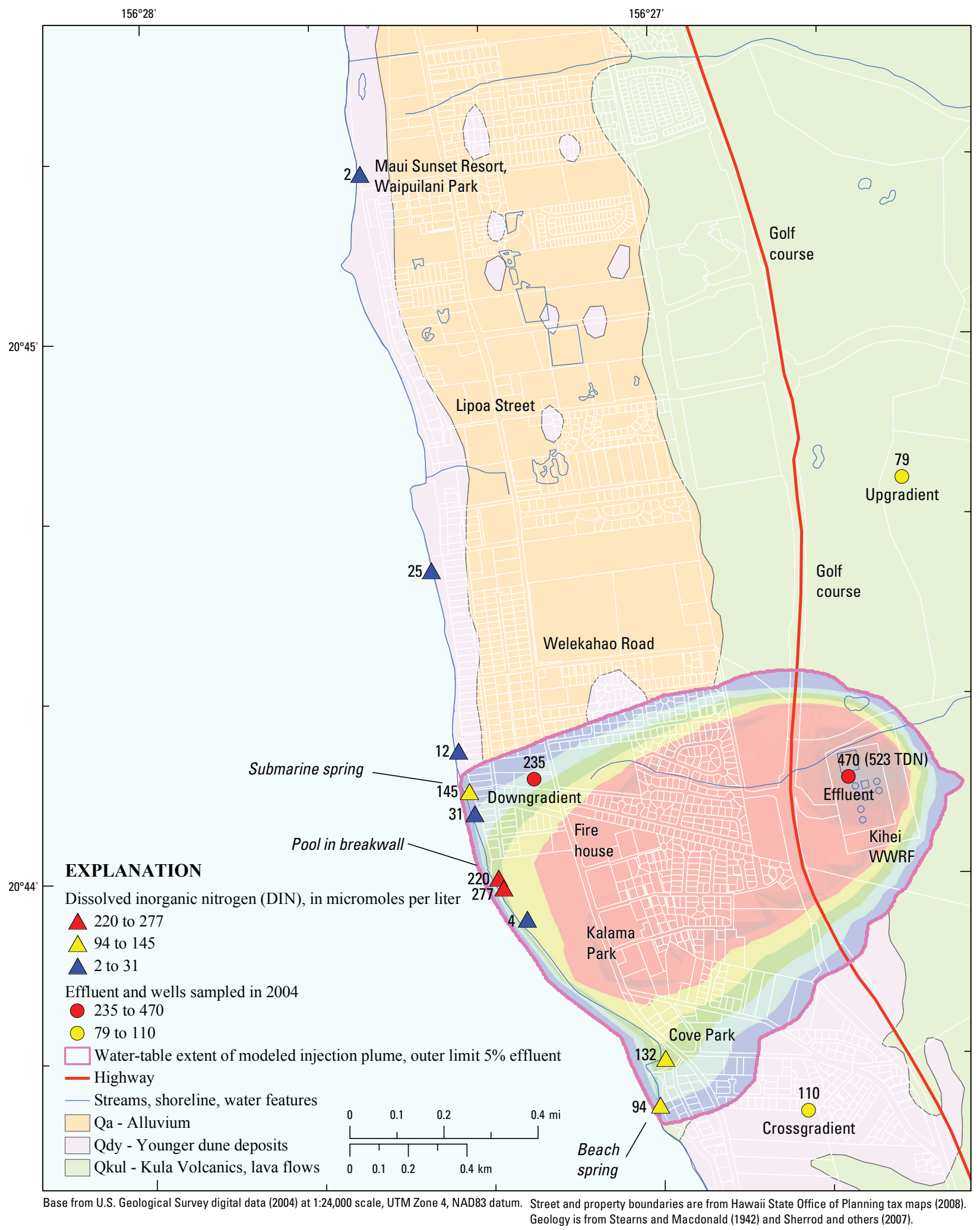

Figure B13. Dissolved inorganic nitrogen (DIN) in water at Kihei, Hawaii. 


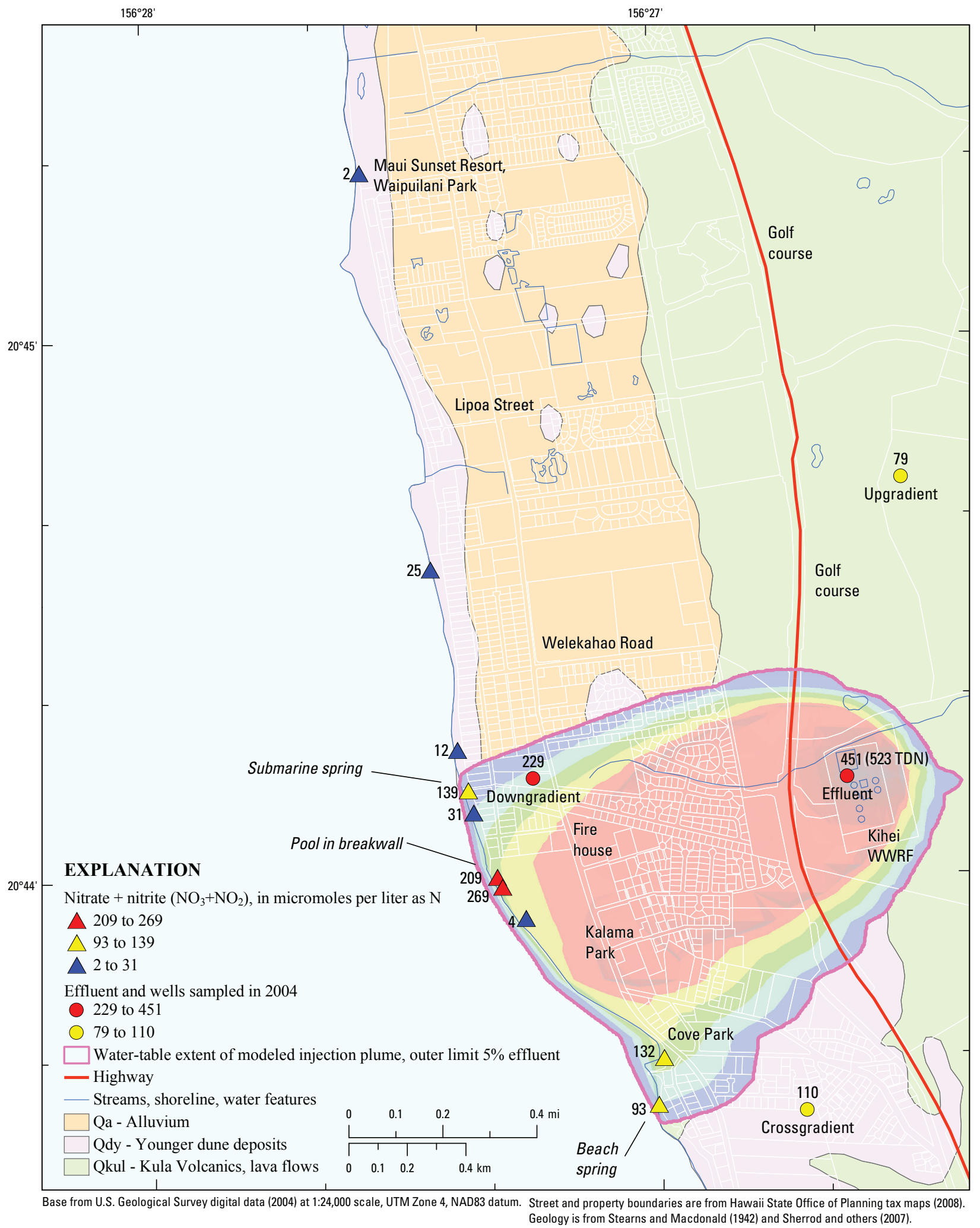

Figure B14. Nitrate plus nitrite in water at Kihei, Hawaii. 


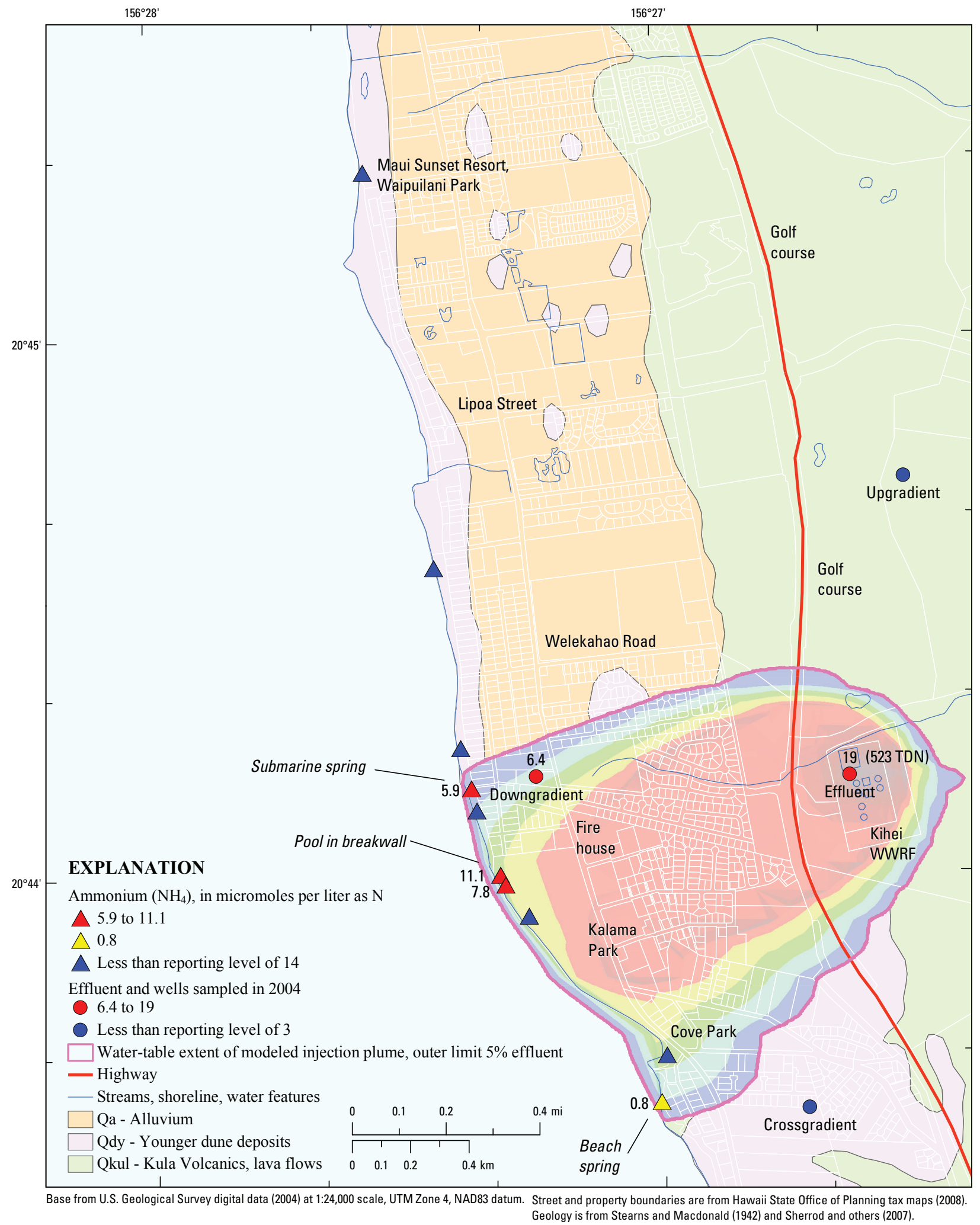

Figure B15. Ammonium in water at Kihei, Hawaii. 


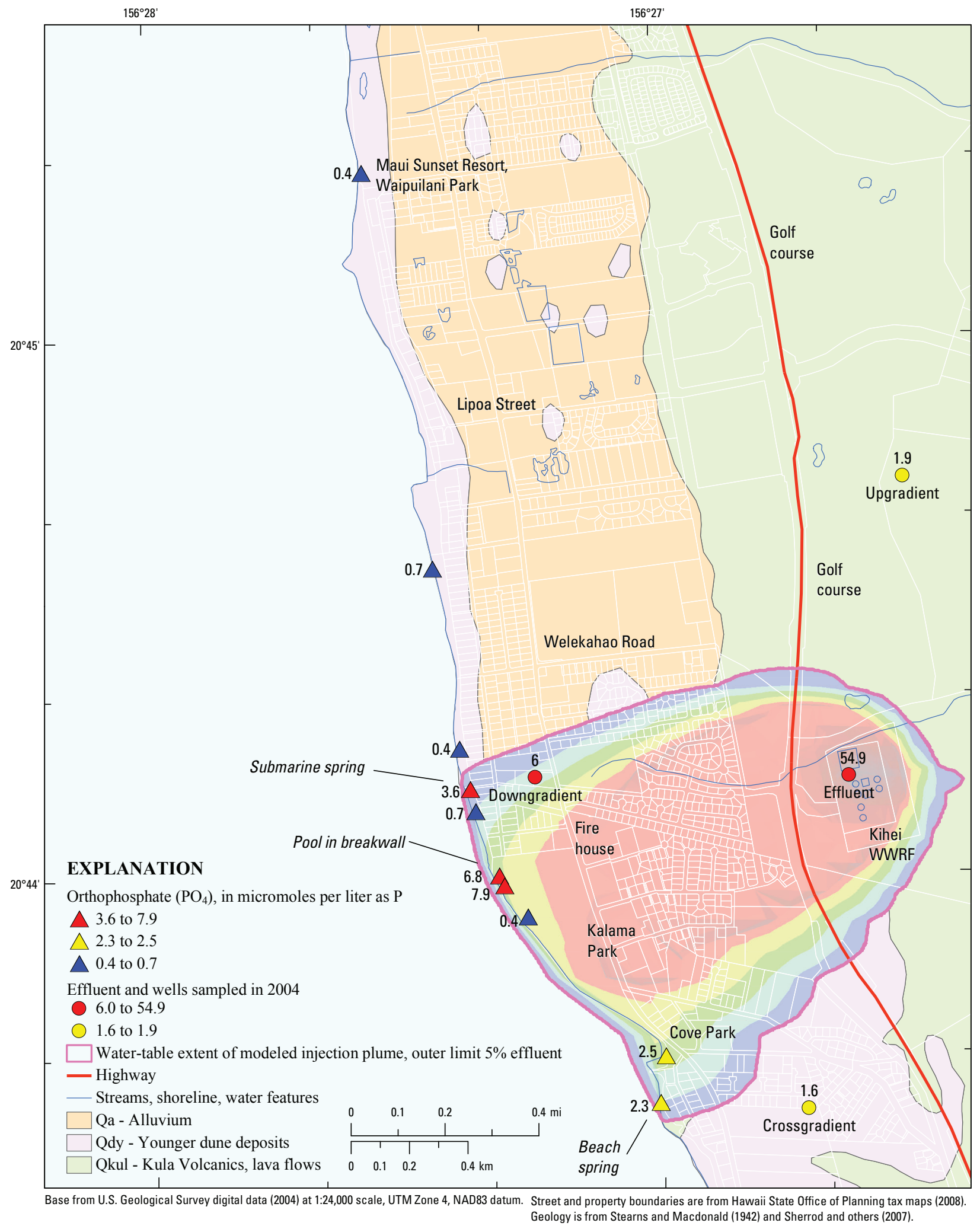

Figure B16. Orthophosphate in water at Kihei, Hawaii. 


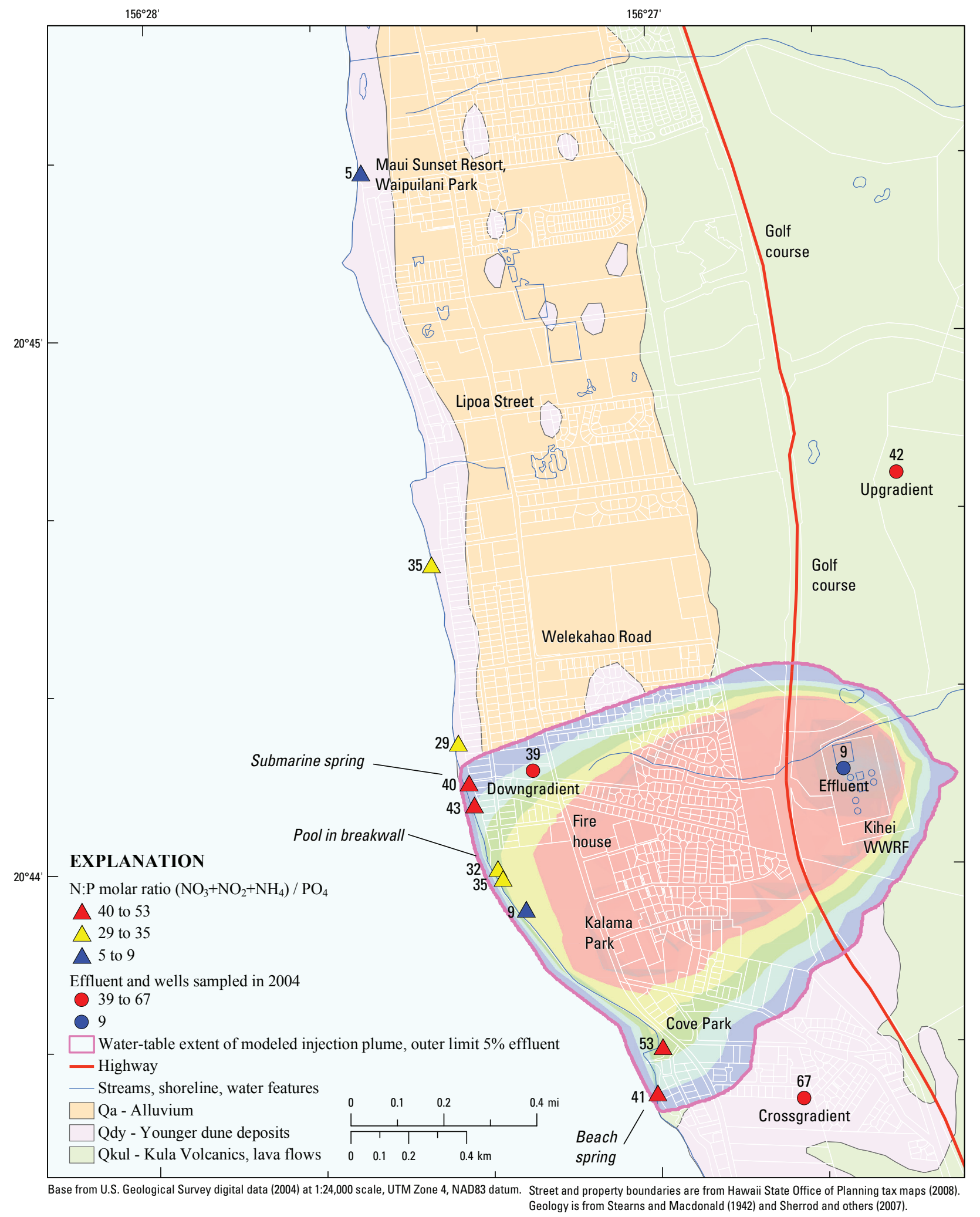

Figure B17. Nitrogen-to-phosphorus molar ratio in water at Kihei, Hawaii. 


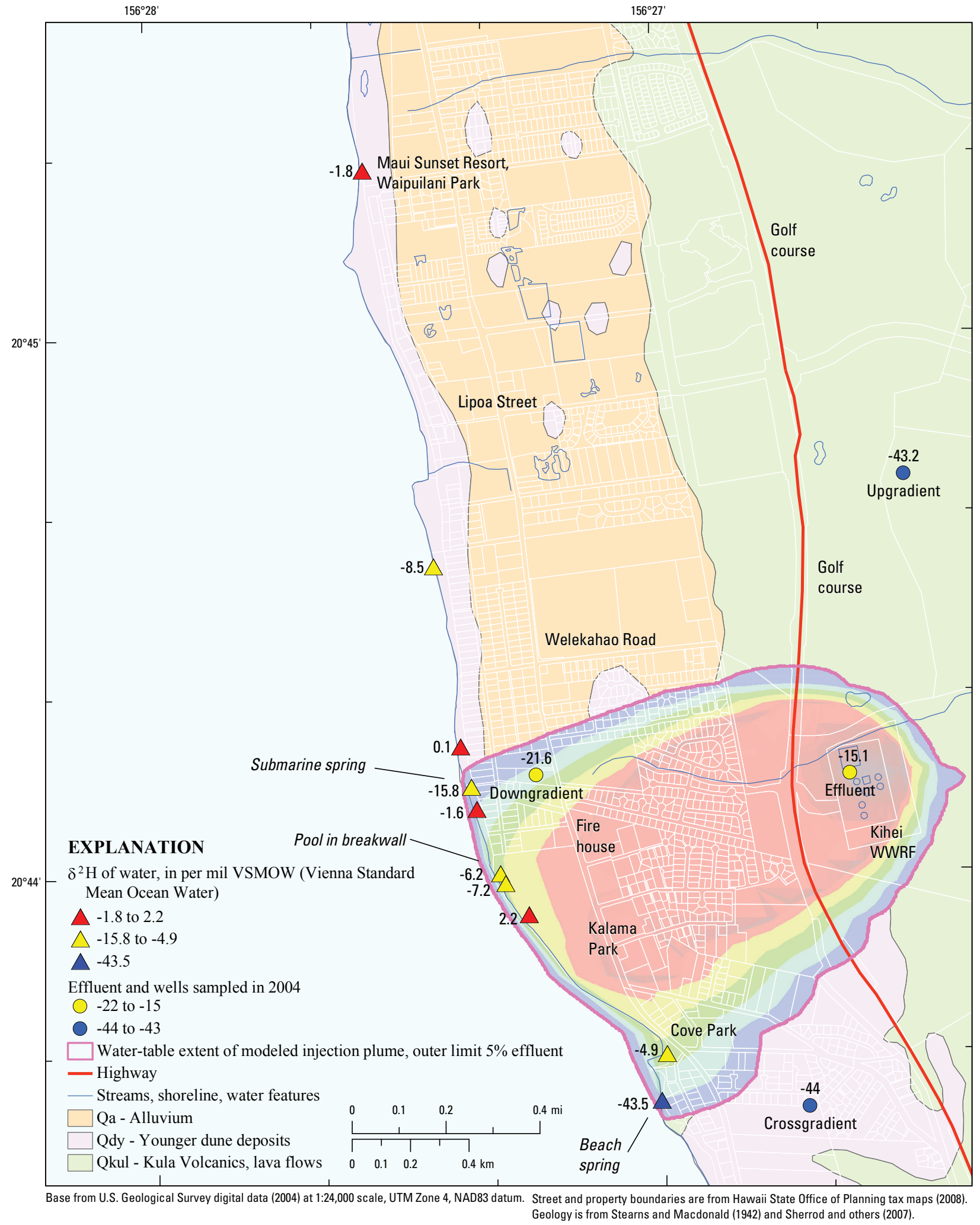

Figure B18. $\delta^{2} \mathrm{H}$ isotopic composition of water at Kihei, Hawaii. 


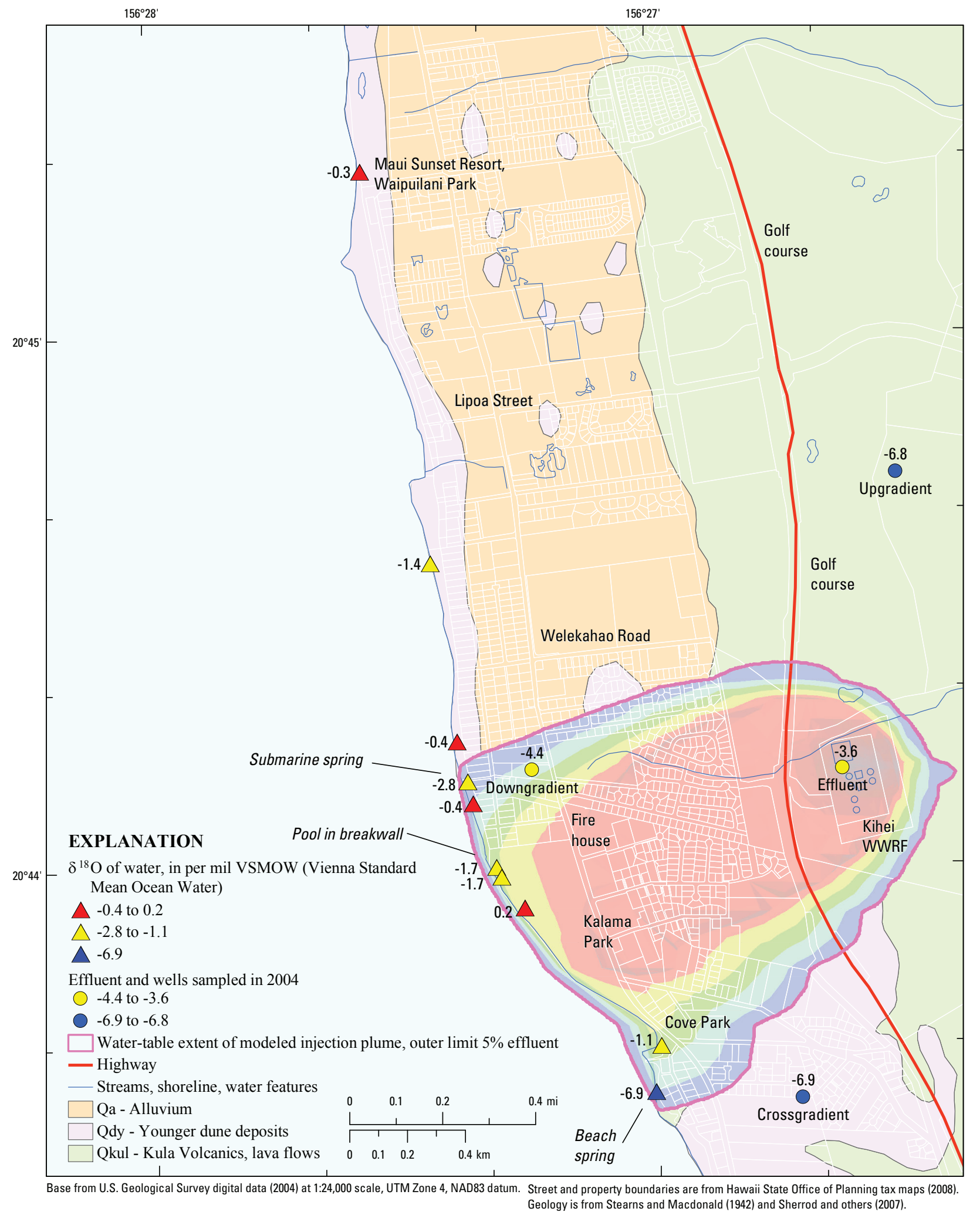

Figure B19. $\delta^{18} \mathrm{O}$ isotopic composition of water at Kihei, Hawaii. 


\section{Appendix C.}

Maps of Laboratory Results and Field Measurements for Water and Algae Samples at Lahaina, Hawaii 


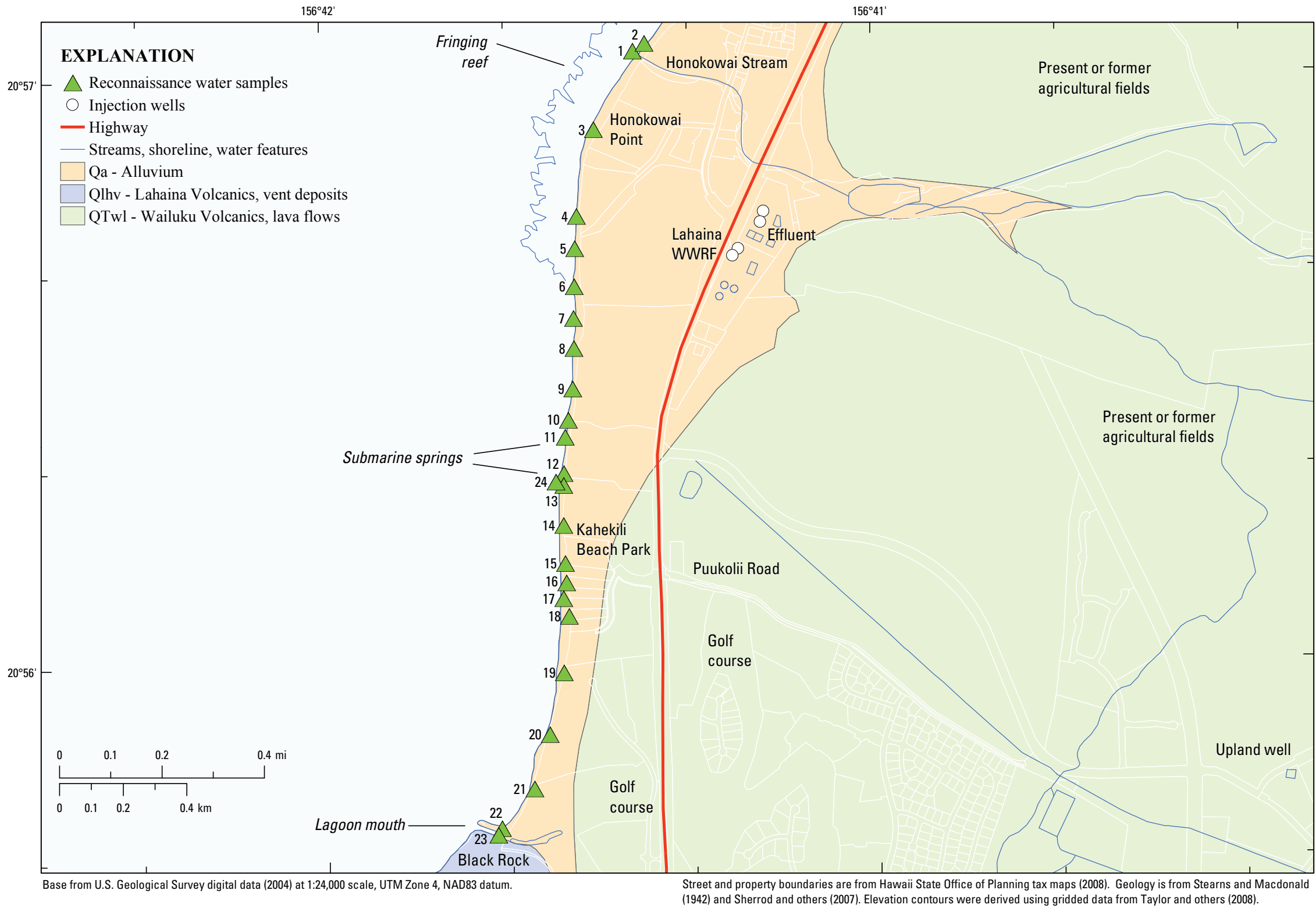

Figure C1. Reconnaissance water samples at Lahaina, Hawaii. 


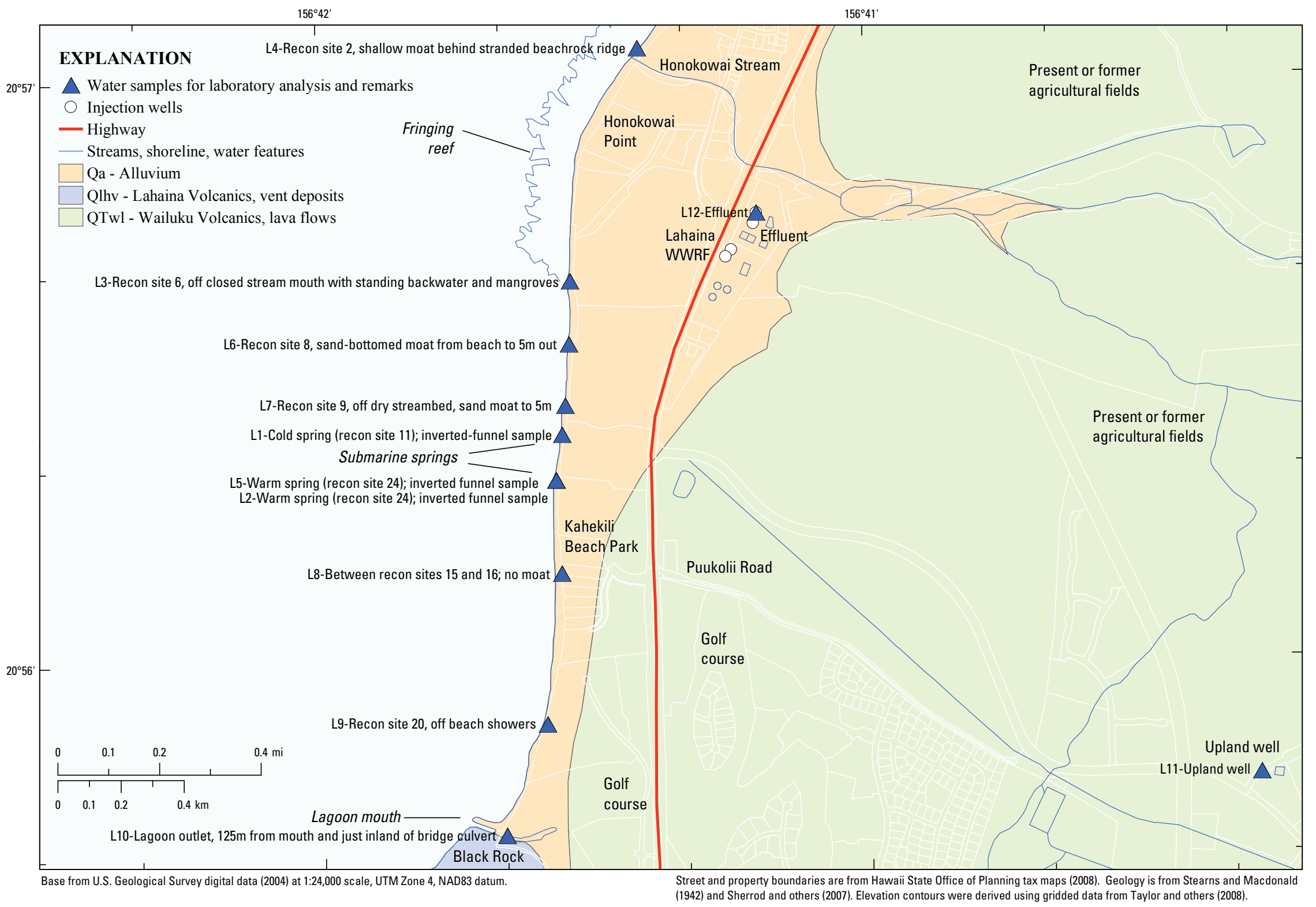

Figure C2. Laboratory water samples (with remarks on their field context) at Lahaina, Hawaii. 


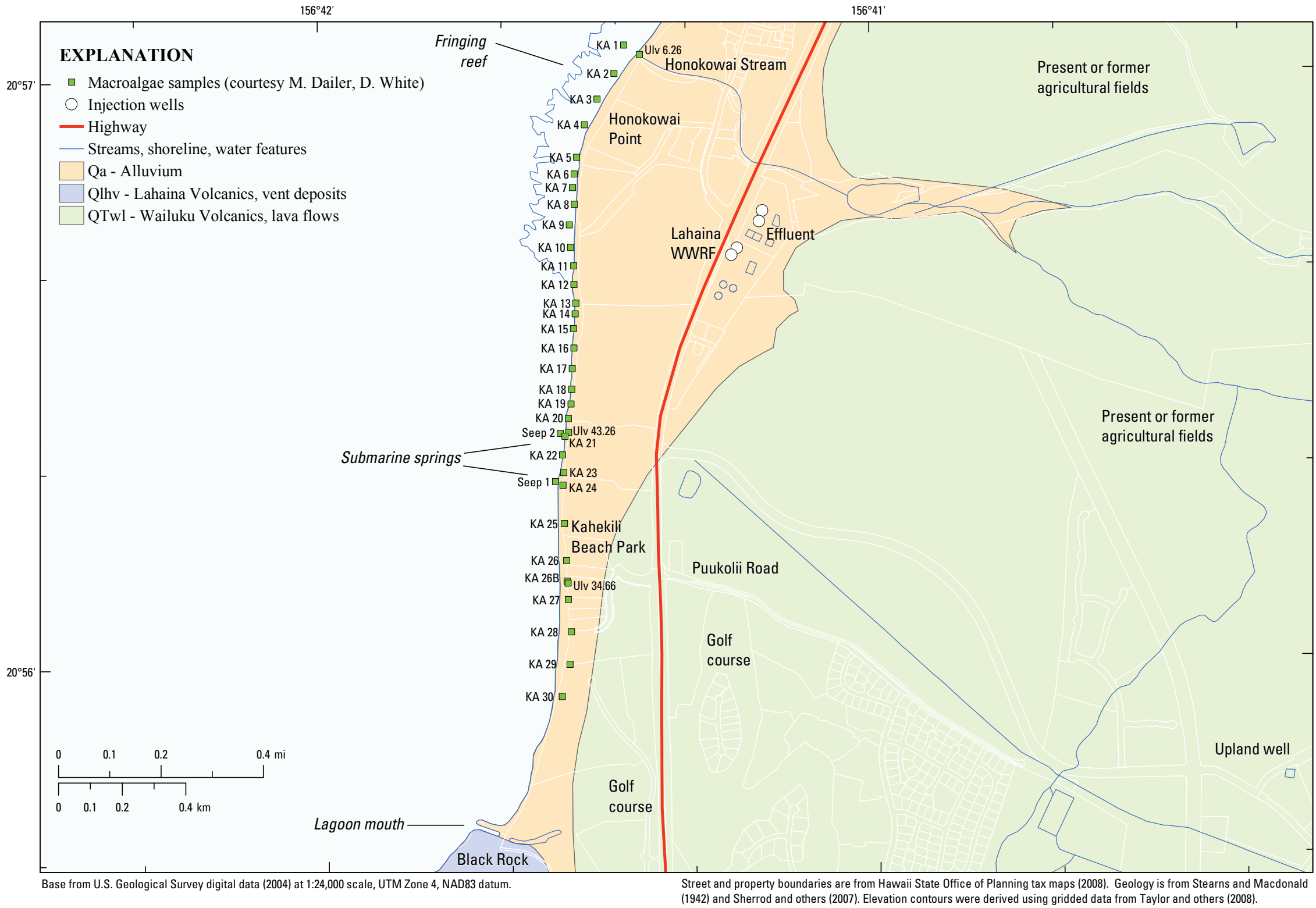

Figure C3. Macroalgae samples at Lahaina, Hawaii. 


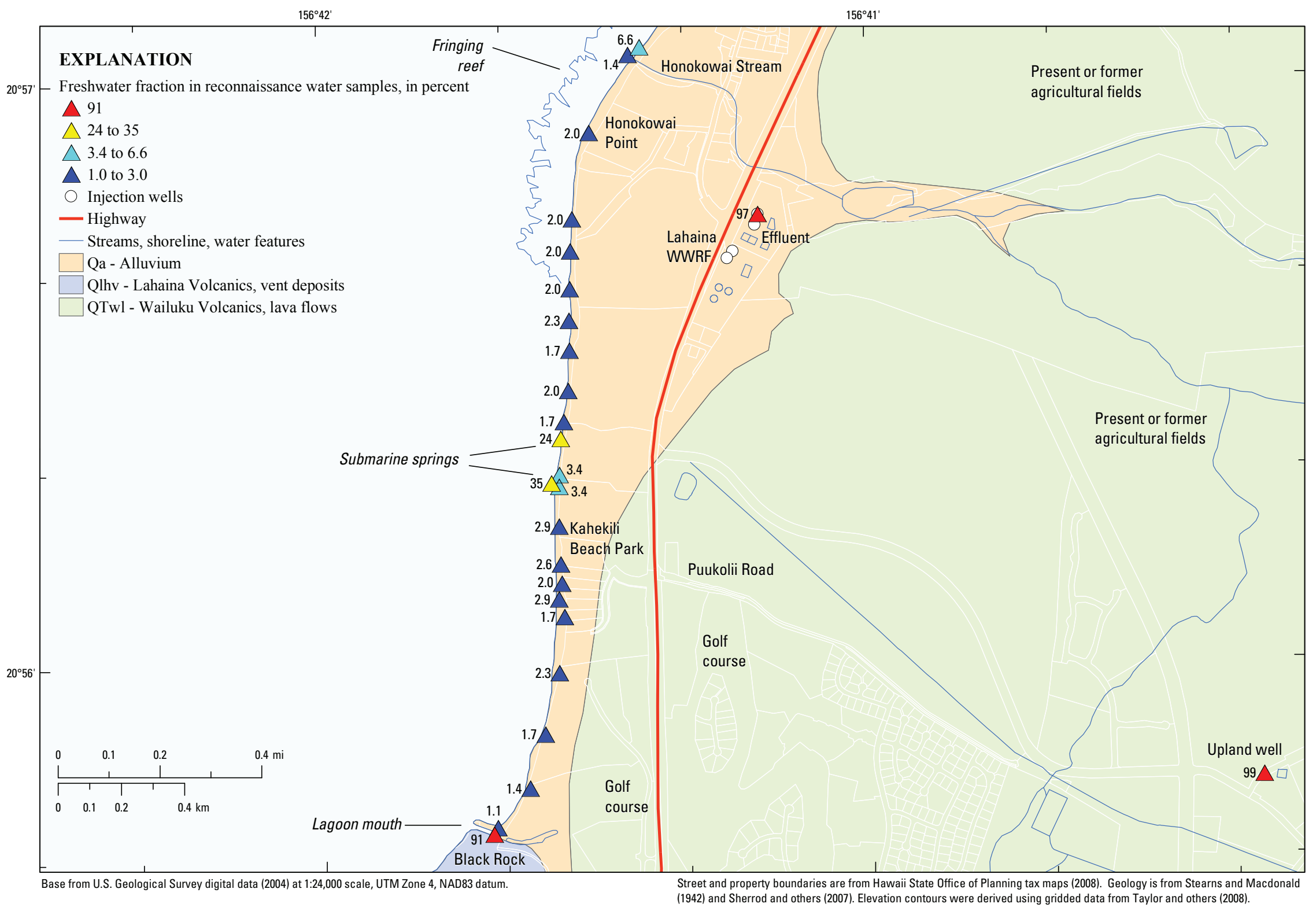

Figure C4. Freshwater fraction (computed from salinity) in reconnaissance water samples at Lahaina, Hawaii. 


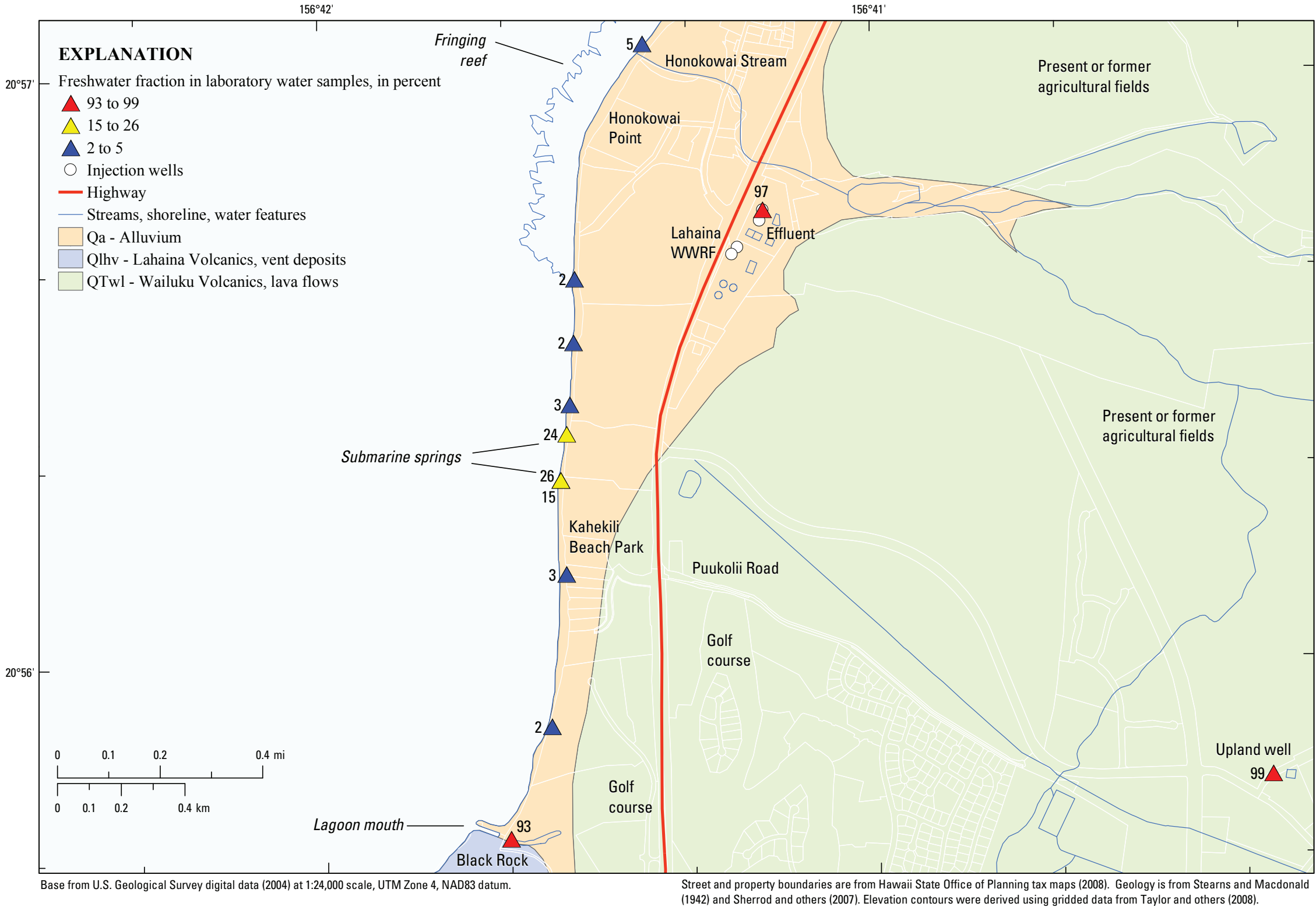

Figure C5. Freshwater fraction (computed from salinity) in laboratory water samples at Lahaina, Hawaii. 


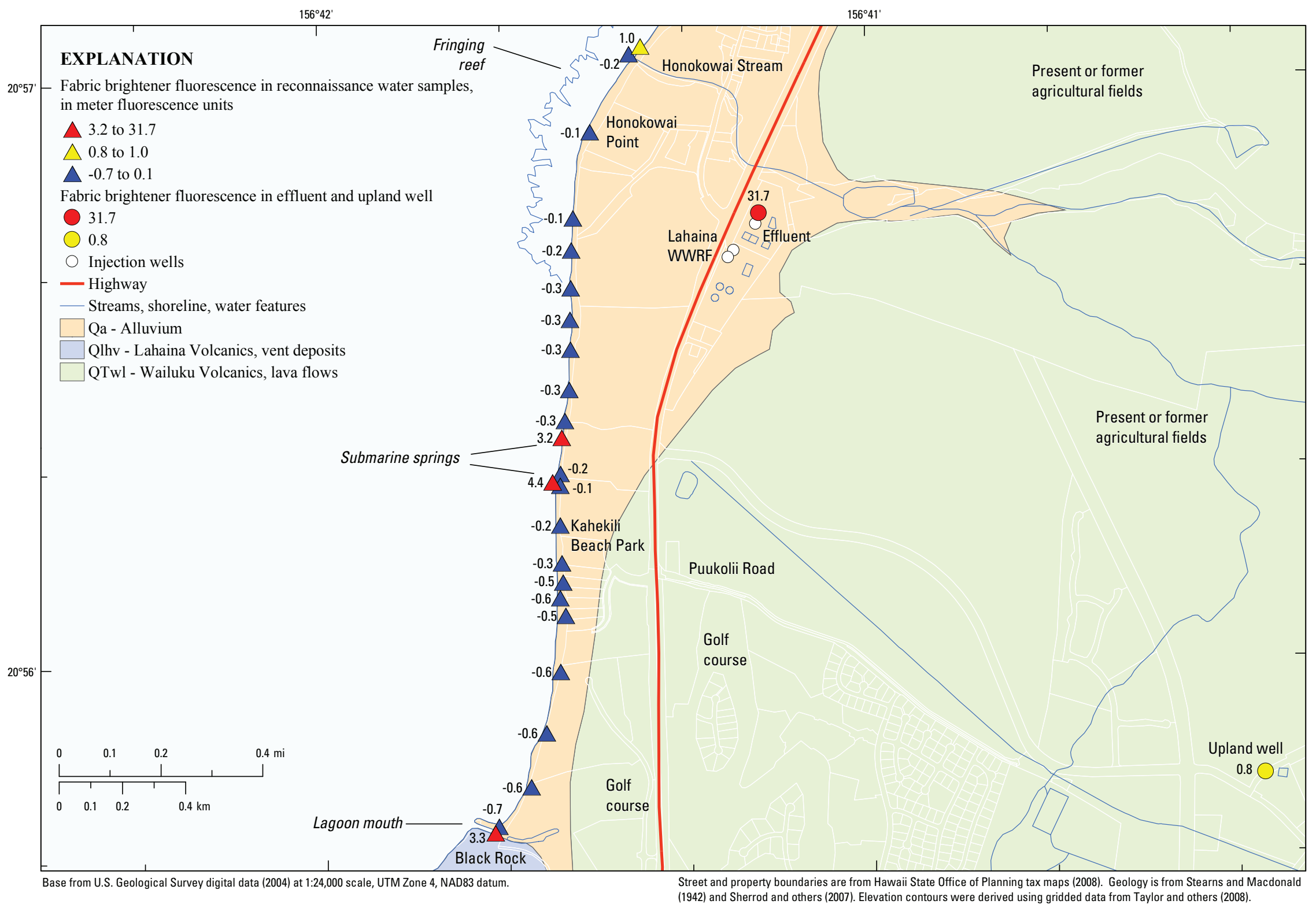

Figure C6. Fabric brightener fluorescence in reconnaissance water samples at Lahaina, Hawaii. 


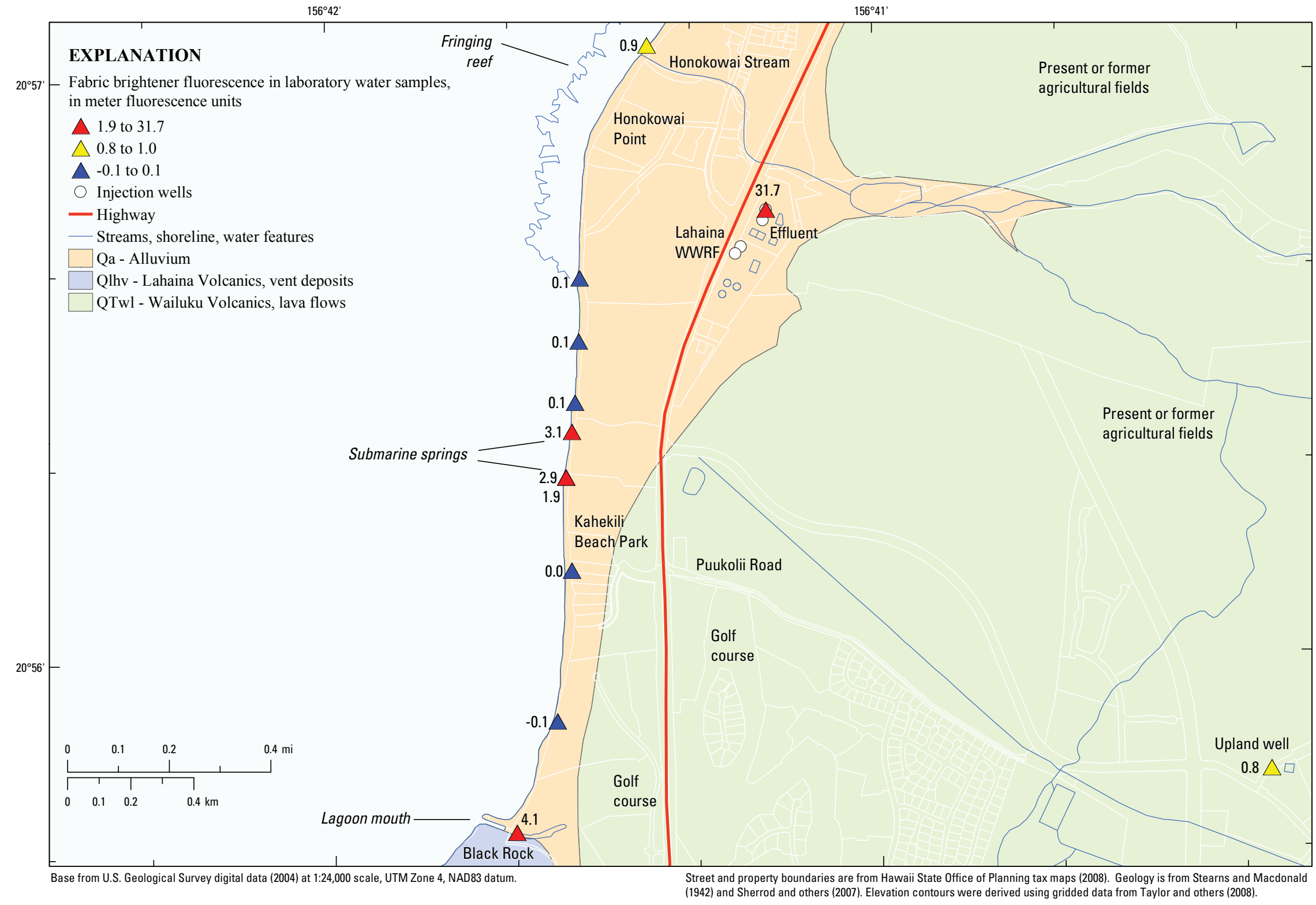

Figure C7. Fabric brightener fluorescence in laboratory water samples at Lahaina, Hawaii. 


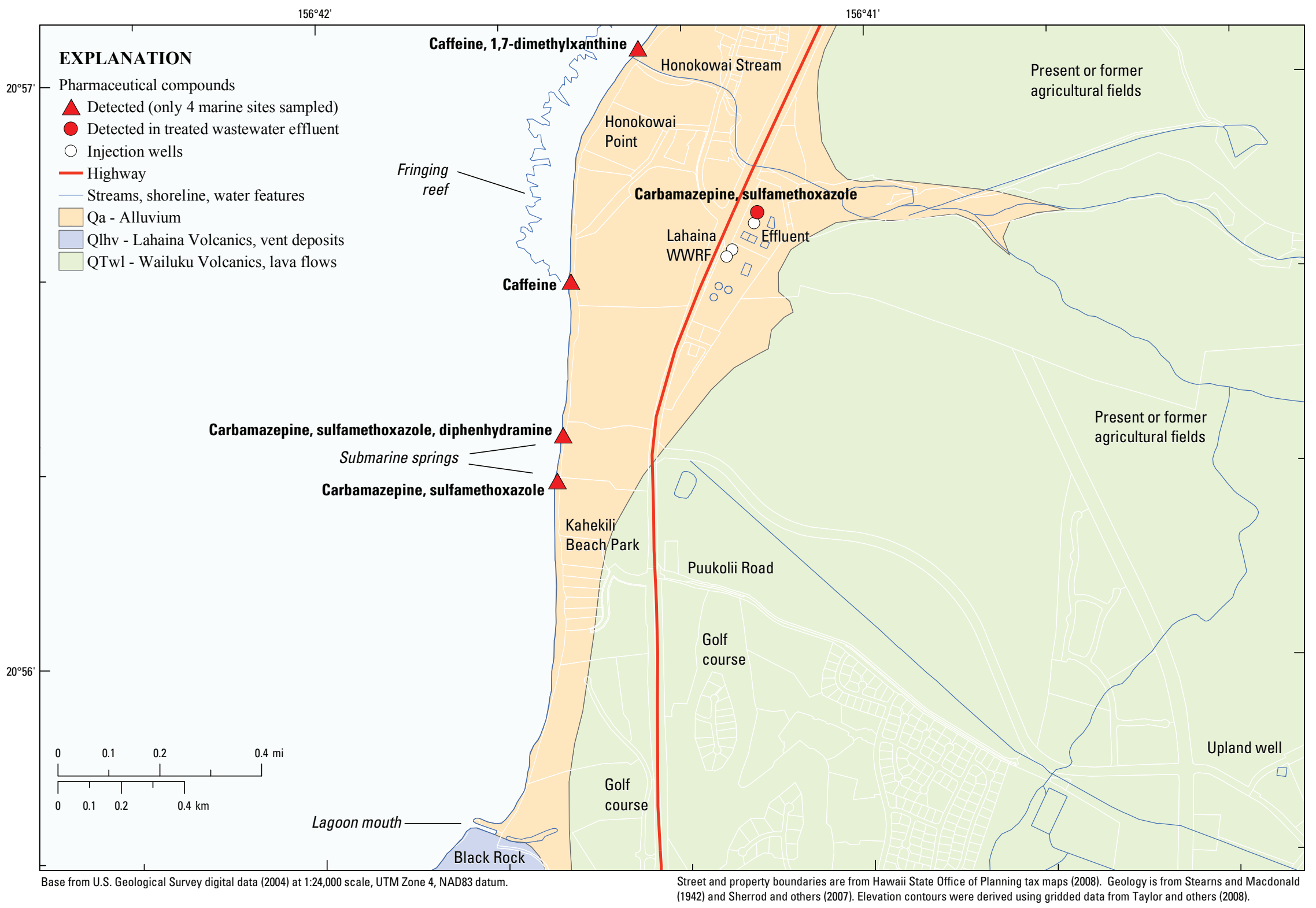

Figure C8. Pharmaceutical compounds in water at Lahaina, Hawaii. 


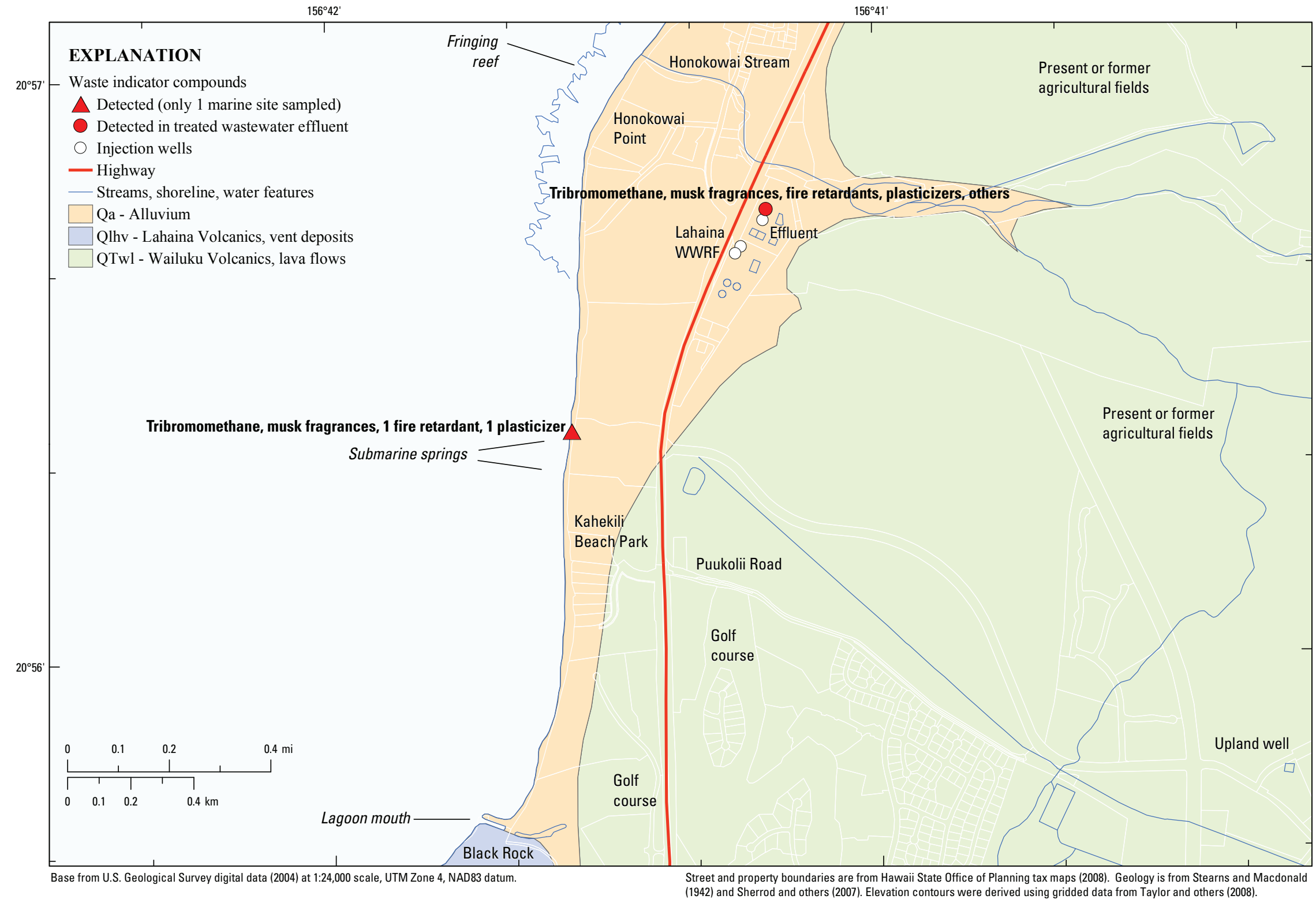

Figure C9. Waste indicator compounds in water at Lahaina, Hawaii. 


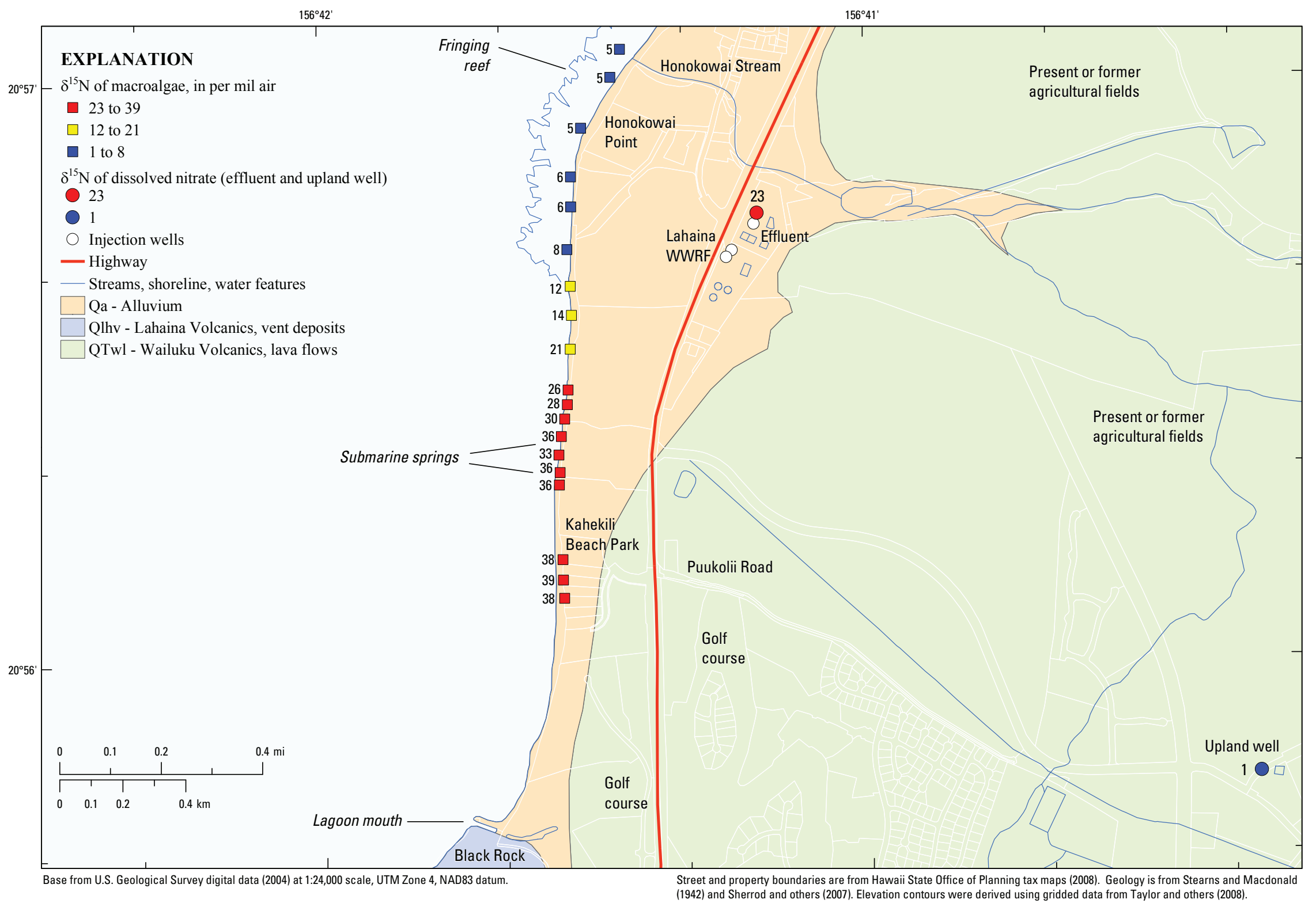

Figure C10. $\delta^{15} \mathrm{~N}$ isotopic composition of macroalgae at Lahaina, Hawaii. 


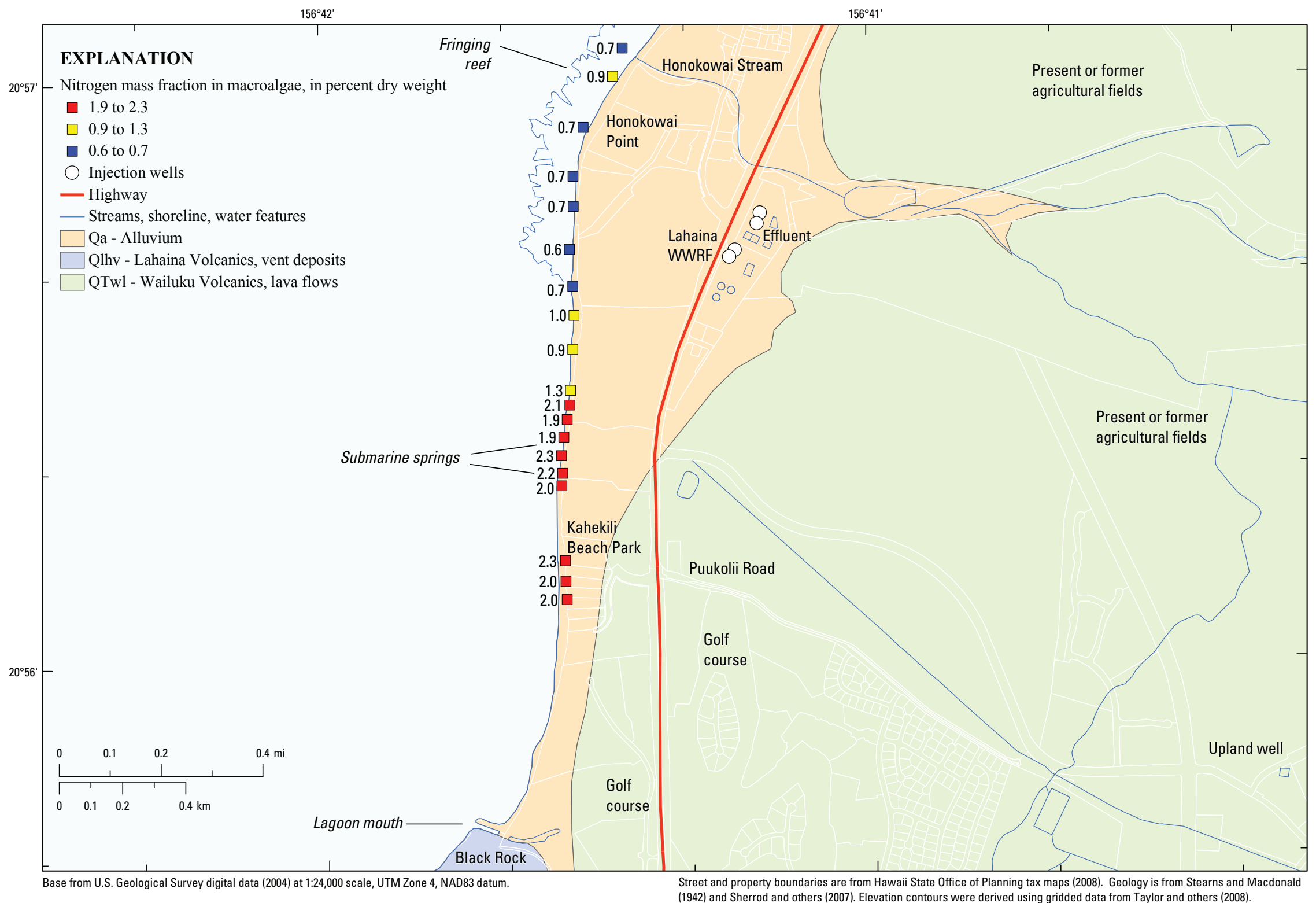

Figure C11. Nitrogen mass fraction in macroalgae at Lahaina, Hawaii. 


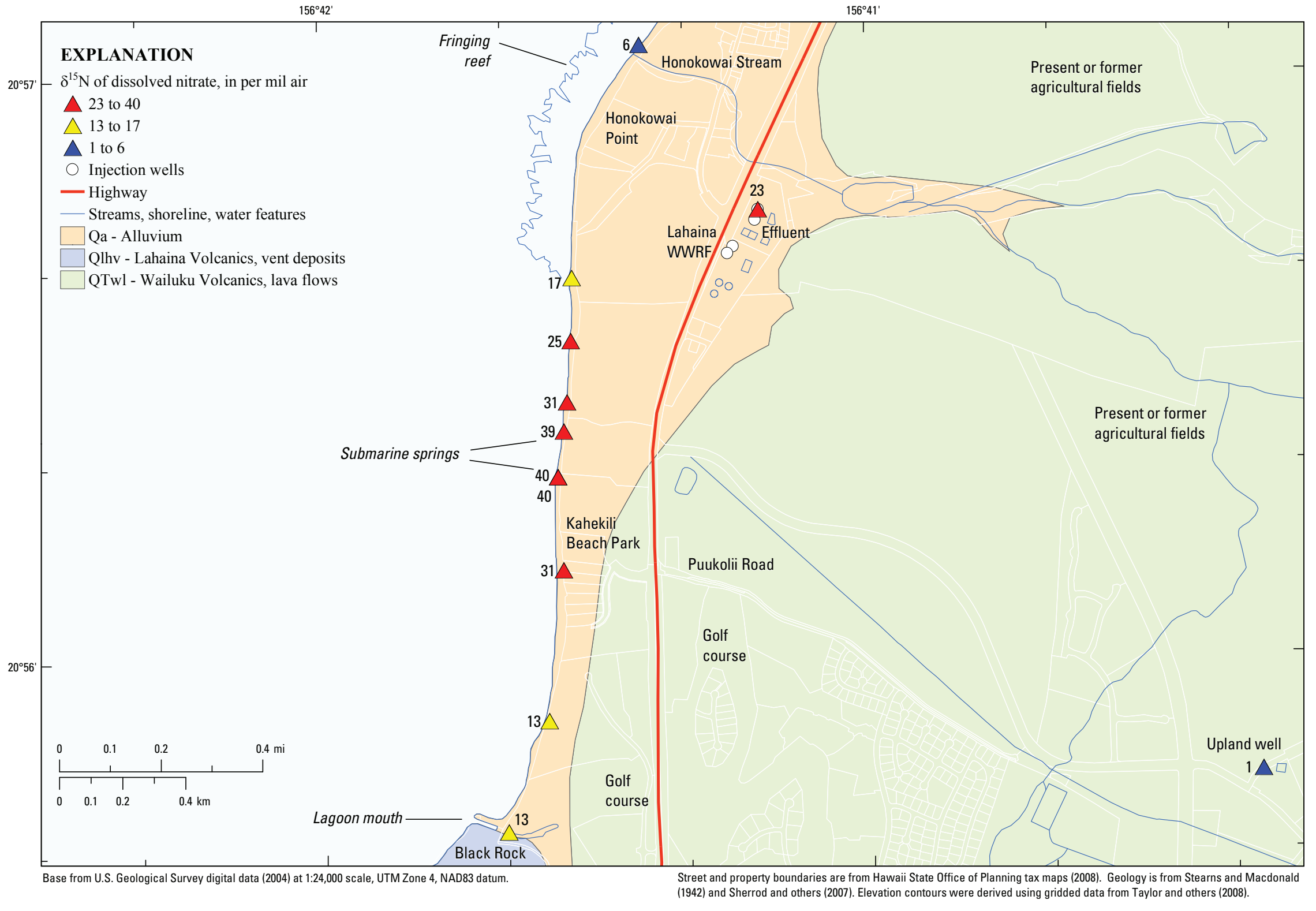

Figure C12. $\delta^{15} \mathrm{~N}$ isotopic composition of dissolved nitrate at Lahaina, Hawaii. 


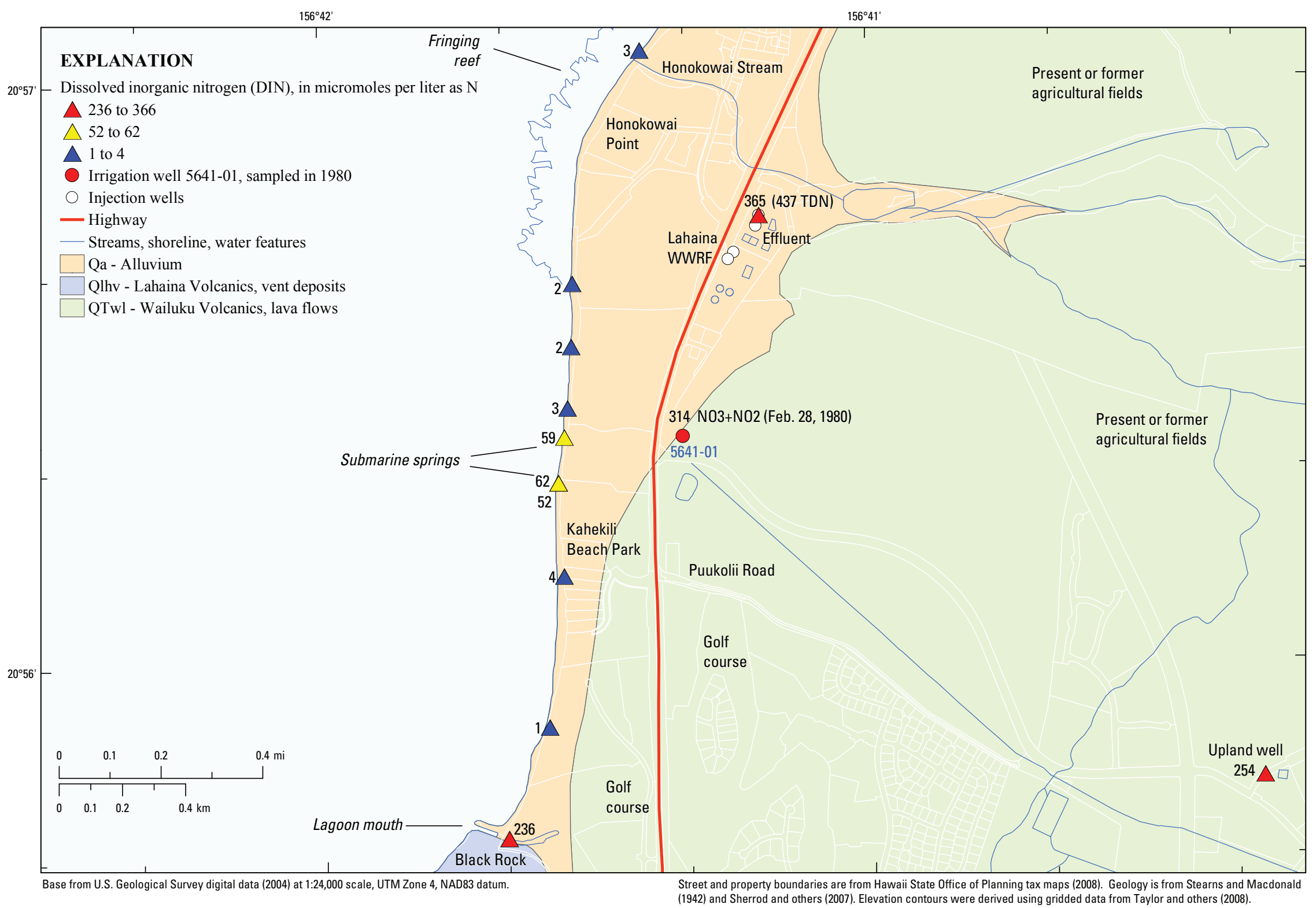

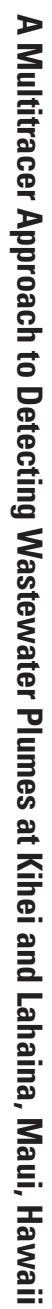

Figure C13. Dissolved inorganic nitrogen (DIN) in water at Lahaina, Hawaii. 


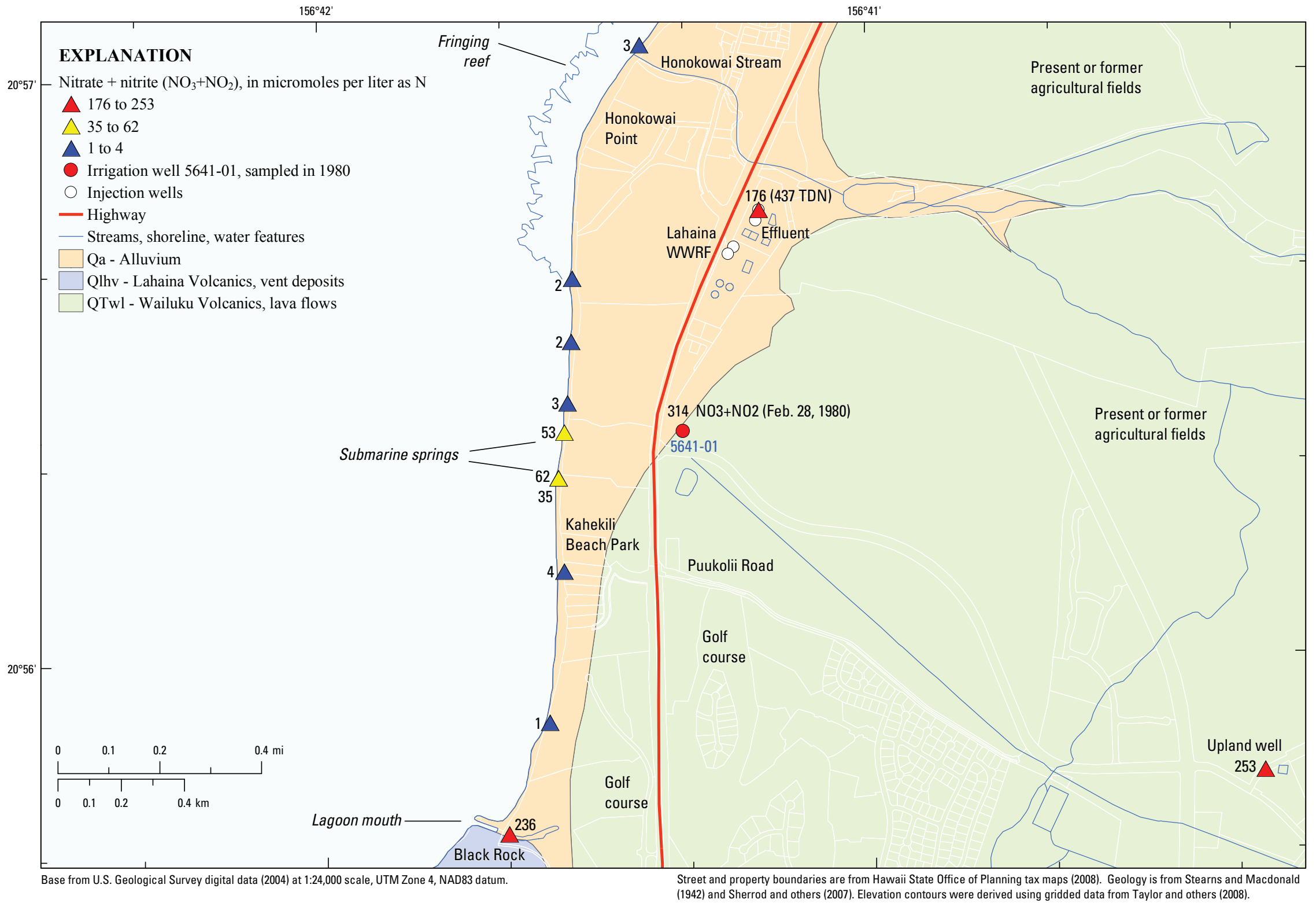

Figure C14. Nitrate plus nitrite in water at Lahaina, Hawaii. 


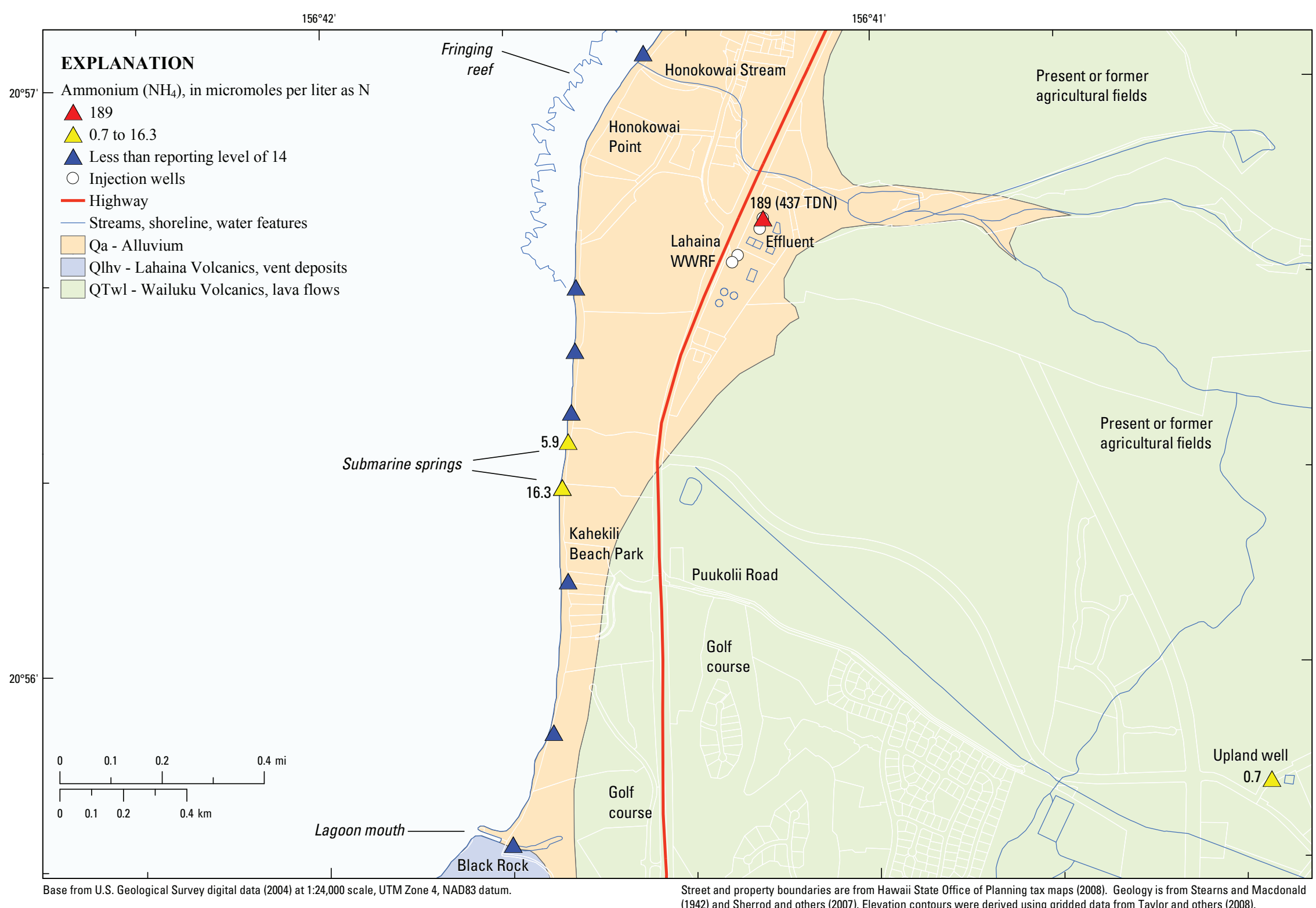

Figure C15. Ammonium in water at Lahaina, Hawaii. 


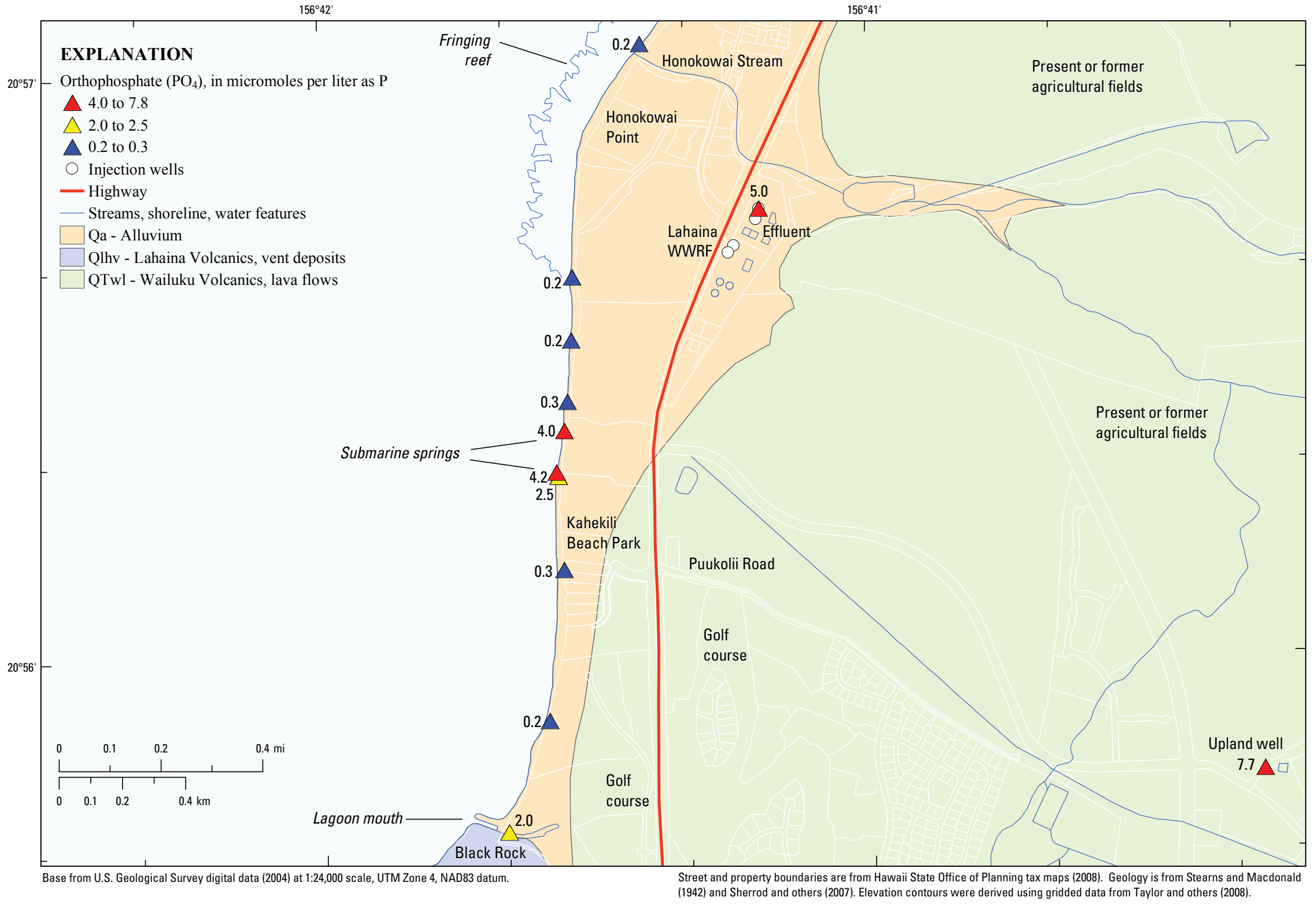

Figure C16. Orthophosphate in water at Lahaina, Hawaii. 


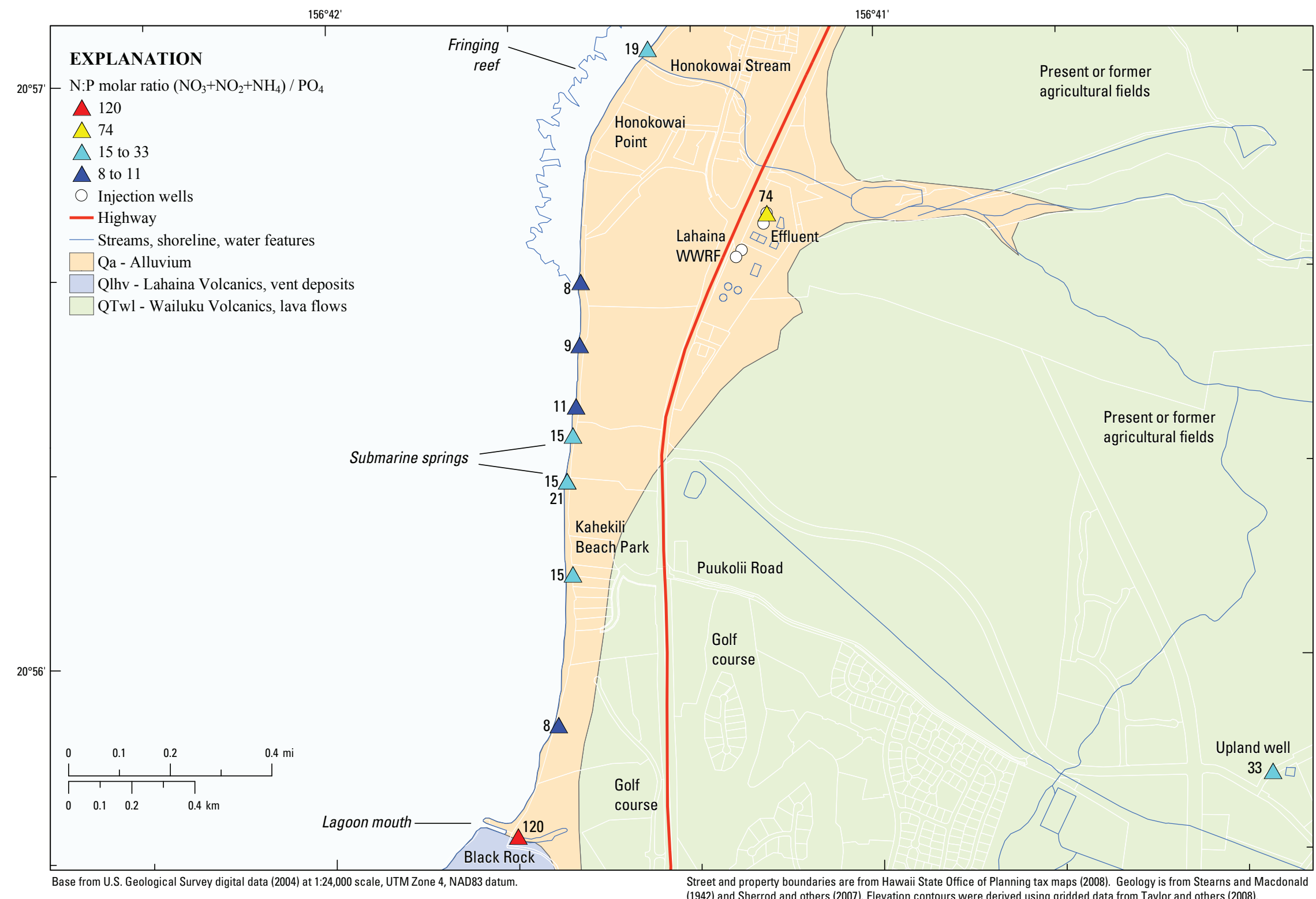

Figure C17. Nitrogen-to-phosphorus molar ratio in water at Lahaina, Hawaii. 


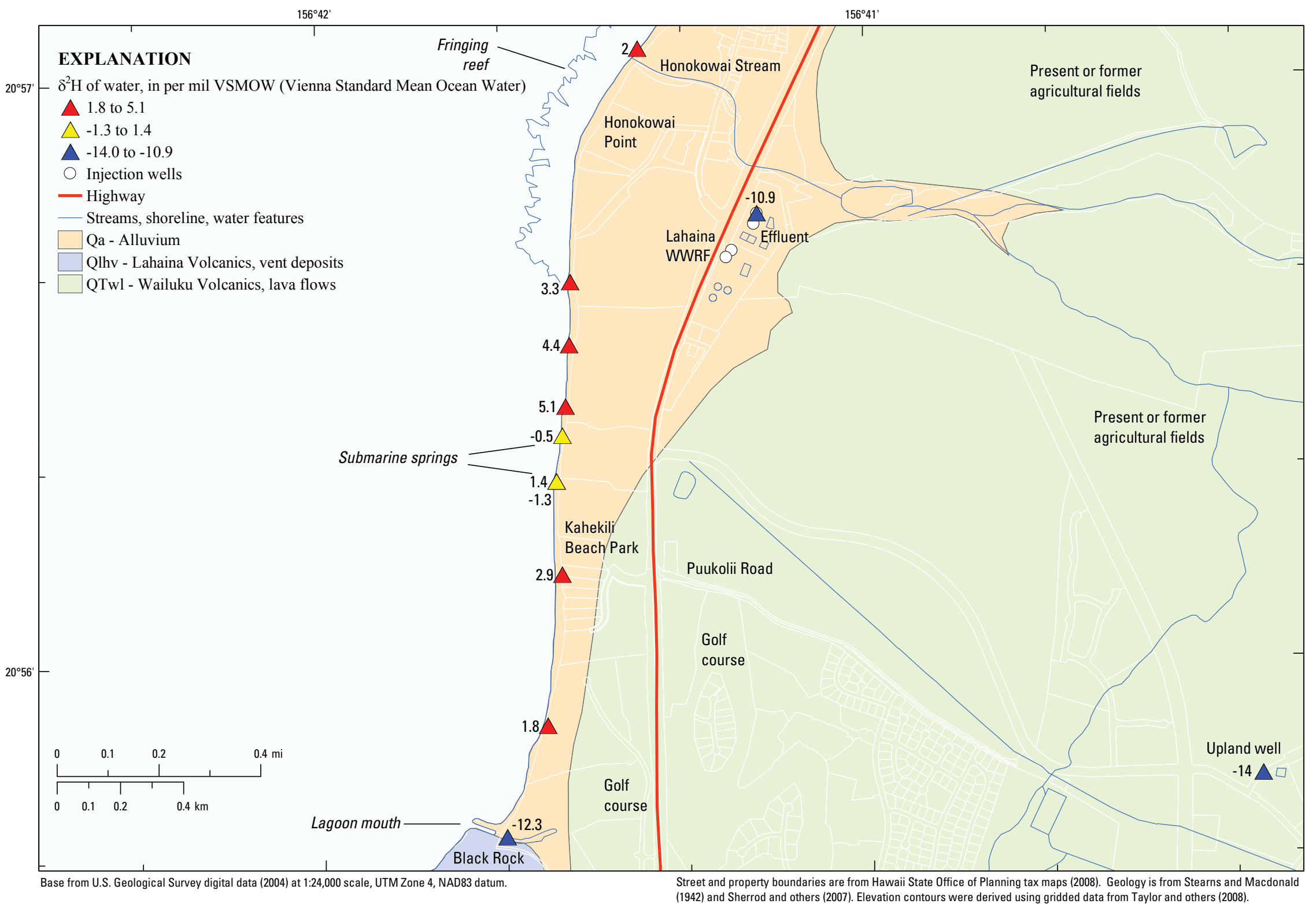

Figure C18. $\delta^{2} \mathrm{H}$ isotopic composition of water at Lahaina, Hawaii. 


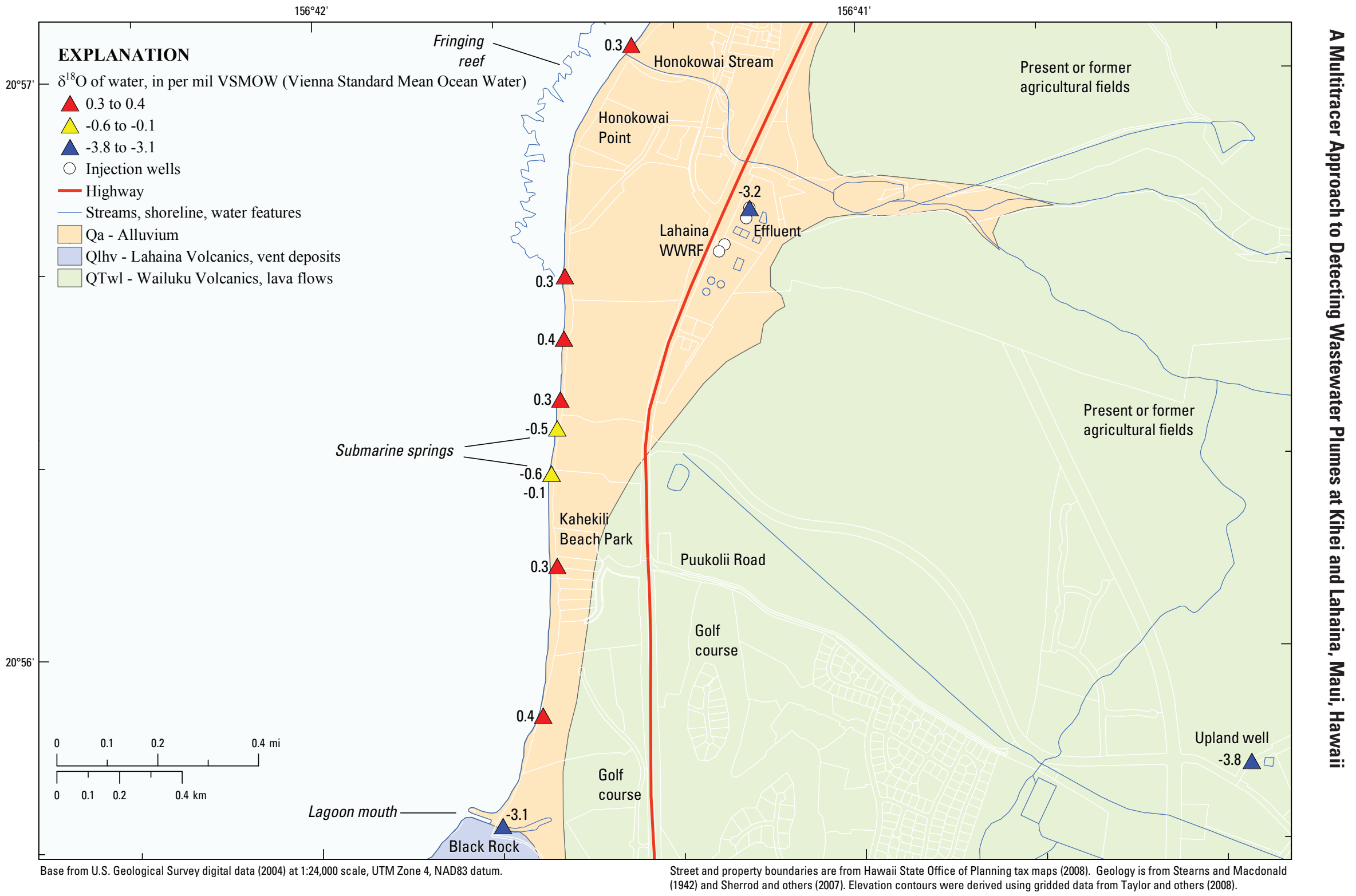

Figure C19. $\delta^{18} 0$ isotopic composition of water at Lahaina, Hawaii. 
Produced in the Western Region, Menlo Park, California Manuscript approved for publication, November 24, 2009 Edited by Peter Stauffer

Layout by David R. Jones 

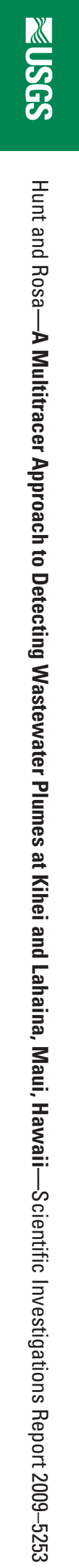